\title{
TRANSMISSIVITY ITERATIVE PROGRAM USER'S MANUAL
}

L. E. Addison, D. R. Friedrichs and K. L. Kipp

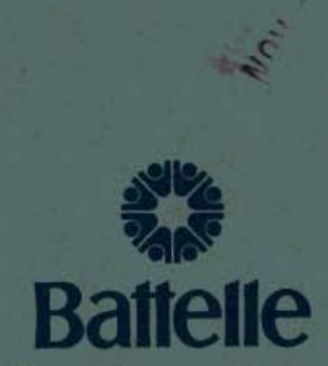

Pacific Northwest Laboratories

Richland, Washington 99352

1972

Prepared for the U.S. Atomic Energy

Commission under Contract AT(45-1):1830 
NOTICE

This report was prepared as an account of work sponsored by the United States Government. Neither the United States nor the United States Atomic Energy Commission, nor any of their employees, makes any warranty, express or implied, or assumes any legal liability or responsibility for the accuracy, completeness or usefulness of any information, apparatus, product, or process disclosed, or represents that its use would not infringe privately-owned rights.

PACIFIC NORTHWEST LABORATORY

operated by

BATTELLE

for the

U.S. ATOMIC ENERGY COMMISSION Under Contract AT(45-1)-1830

\author{
Printed in the United States of America \\ Available from \\ National Technical Information Service \\ U.S. Department of Commerce \\ 5285 Port Royal Road \\ Springfield, Virginia 22751
}

Price: Printed Copy $\$ 3.00$; Microfiche $\$ 0.95$ 


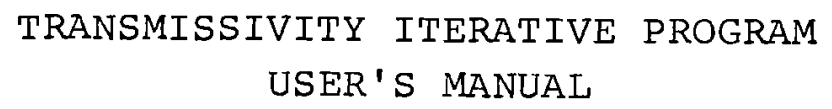

by

L. E. Addison

D. R. Friedrichs

K. L. Kipp

Water and Land Resources Department

This report is based on work sponsored by the Atlantic Richfield Hanford Company under United States Atomic Energy Commission Contract AT (45-1)-2130 


\section{SUMMARY}

This user's manual contains listings and functional definitions of the various directives available in the Iterative Sequence. These directives are used to operate the Iterative Sequence and to direct and complete the actual analysis. A complete and thorough description of each directive is contained in the report, "The Transmissivity Iterative Programs on the PDP-9: A Man-Machine Interactive System," BNWL-1707. 


\section{CONTENTS}

$\underline{\text { Page }}$

SUMMARY • . . . . . . . . . . . . . . . . . . . . . . . . . . . . . .

INTRODUCTION

ITERATIVE SEQUENCE OPERATING INSTRUCTIONS • • • • . . • 3

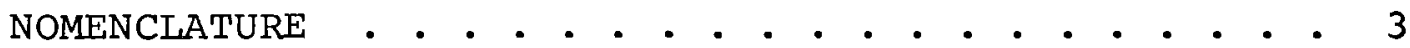

ITERATIVE MONITOR (KONTRL) . • . . . . . . . . 3

STREAMLINE GENERATION (STREAM) • • • • • • • . . • • 7

TRAVEL TIME CALCULATIONS (TRAVPR, TRAVTR) • • • • • . 9

TRANSMISSIVITY CALCULATIONS (FLOWSS, FLOWTR) . . . 10

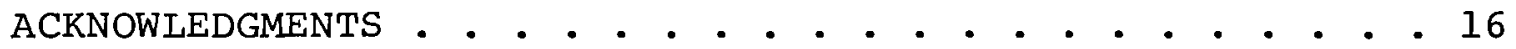

APPENDIX A - EXECUTIVE PROGRAM LISTING. . . . . . . . . A-1

APPENDIX B - STREAMLINE PROGRAM LISTING . . . . . . . . . B-1

APPENDIX C - TRAVEL TIME PROGRAM LISTING. . . . . . . . C-1

APPENDIX D - TRANSMISSIVITY PROGRAM (STEADY STATE)

LISTING. • • • • • • • • • • • • • • • . D-1

APPENDIX E - TRANSMISSIVITY PROGRAM (TRANSIENT STATE)

LISTING. • • • • • • • • • • • . . . . E-1 


\section{TRANSMISSIVITY ITERATIVE PROGRAM \\ USER'S MANUAL}

\section{INTRODUCTION}

This user's manual describes the operation of the set of programs developed to calculate the transmissivity distribution for the Hanford Reservation and perform travel time studies. Two other reports contain the theory upon which these programs are based and the details of the computer system and program set design.

The program set includes:

1. the Iterative Monitor;

2. Streamline Generation program;

3. Travel Time programs; and

4. Transmissivity Calculation programs.

A description of the operations involved in generating the transmissivity distribution using this program set on the interactive computer system is covered in a separate report, "The Transmissivity Iterative Programs on the PDP-9 Computer - A ManMachine Interactive System," BNWL-1707. 


\section{ITERATIVE SEQUENCE OPERATING INSTRUCTIONS}

This report is a detailed summary of the available options and operating instructions for each program in the Iterative Sequence.

NOMENCLATURE

Directives The input neumonic to the computer which directs the computer to perform a certain task. Directives are three characters long.

TAG

The use tag for a data matrix; identifies the usage of a particular data matrix.

DEV

Any of the mass storage devices (disc and dectape) on this system. The device neumonic is three characters in either DKU or DTU, where $U$ is the proper unit number of the device.

NAME

The directory name for a data file, 1 to 6 characters in length.

EXT

The directory extension for the data file, 1 to 3 characters in length.

TIME

The difference in time between time 2 and time 1 expressed in days. It must be positive and no larger than 131,072 days (359.1 years).

FUNCTION The functional block to which the Iterative Monitor dispatches control.

ITERATIVE MONITOR (KONTRL)

The Iterative Monitor acts as a general supervisor for the Iterative sequence, performing data retrieval and storage, initialization, certain data matrix operations, and dispatch of control to the proper functional block in the Iterative Sequence. All of the directives for the Iterative Monitor are input from the keyboard of the ASR35 teletype.

When the monitor is first called for execution, it responds: 


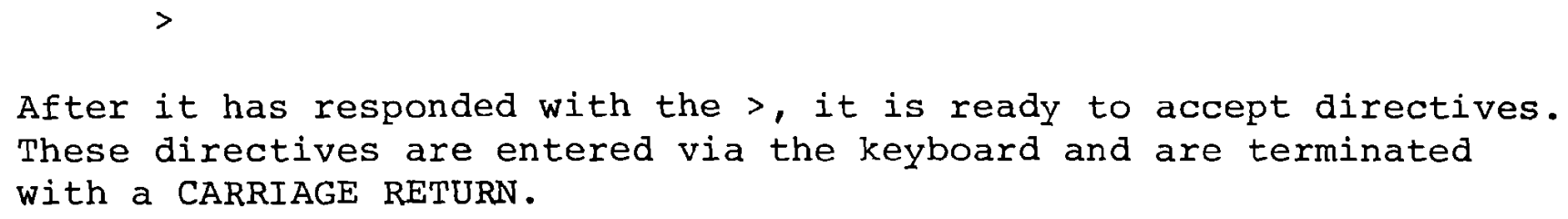

Directives

INT
GEN TAG NAME EXT

\section{Task}

The graphic digitizer table is to be initialized. When this directive has been given, the computer will respond on the teletype

\section{DIGITIZE $(0,0)$ POINT}

The computer is requesting that the digitizer cursor be placed at the $(0,0)$ coordinate of the reference data map being used. When the cursor has been placed at the point, the button on the digitizer cursor is pressed to send the point to the computer. The computer will respond

\section{DIGITIZE MAXIMUM X VALUE}

The digitizer cursor should now be placed at the maximum $x$ node on the reference data map being used and the button on the digitizer cursor should be pressed to enter the point into the computer.

This directive establishes a new data matrix NAME EXT on disc unit 2 (DK2). It is filled entirely with zeros and is assigned to the use TAG.

After this directive has been given the computer will require the following additional information to set up this new data matrix:

HORIZONTAL DIMENSION (nodes in horizontal direction) 
Directives

GEN TAG NAME EXT

(continued)

ASG TAG DEV NAME EXT

DEL TAG

SAV TAG DEV NAME EXT

LST

COM TAG $\underline{\text { Task }}$

VERTICAL DIMENSION (nodes in vertical direction)

SECTORS PER SECTION (number of disC sectors per section)

OVERLAP (number of nodes in common)

(For a detailed explanation of these parameters, see Appendix III in a separate report, BNWL-1707).

This directive transfers a data matrix (NAME, EXT) from the physical device (DEV) to disc unit 2 (DK2). After the transfer has been completed, the matrix is assigned to use (TAG). This directive overlays any previous assignment to (TAG).

Delete the current data matrix assigned to TAG.

Transfer the data matrix assigned to use (TAG) on disc unit 2 (DK2) to the physical device (DEV). The data matrix is identified on the device (DEV) by (NAME, EXT). If there currently is a file on (DEV) by that identification, it is deleted and this replaces it. If (NAME EXT) are not included in the directive, then the same name as in the assignment (TAG) will be used.

List on the teletype the data matrix use assignments as they are currently specified.

Enter in the data matrix assigned to use (TAG) a comment string. This comment string is limited to 1 teletype line (64 characters). When this directive is given the computer will respond on the teletype with

COMMENTS 
Directives

COM TAG

(continued)

TIM

EXQ FUNCTION

AVE

DIF

FIL TAG

SMU TAG

FILE NOT FOUND

DISC ERROR

Special Keys

$\uparrow U$ (Control U)

Rub Out
Task

The desired comments are entered via the keyboard and stored in the data matrix.

This sets the time value between $\mathrm{T} 2$ and Tl to Time.

Dispatch control to the function (FUNCTION)

Form the average of the two data matrices assigned to use (PTI) and (PT2) and store the results in the data matrix assigned to use (PTA). (PTA) $=[(\mathrm{PT} 2)+(\mathrm{PT} 1)] / 2$

Form the difference between the data matrix assigned to use (PT2) and the data matrix assigned to use (PTI) and store the results in the data matrix assigned to use (DPT). $(\mathrm{DPT})=(\mathrm{PT} I)-(\mathrm{PTl})$

Fill all of the internal zeros in the data matrix assigned to use (TAG).

Smooth the data matrix assigned to use (TAG).

A file identifier (NAME EXT) was given which was not found on the physical device (DEV).

An error has been detected in the disc transfers or operation.

This key deletes everything previously typed on this directive; it is as if nothing had been typed. All previous input on this directive is ignored.

This deletes the last character typed from the current directive being input. successive rub outs continue to delete the successive previous input character. 
Errors Detected by Monitor

?

\author{
An error was made on directive input; \\ for example: \\ 1. illegal character \\ 2. improper input of a directive \\ 3. illegal device \\ 4. illegal use tag
}

STREAMLINE GENERATION (STREAM)

This functional block generates streamlines and draws contours for a groundwater system. The directives for Streamline are entered either at the graphic digitizer or at the console of the CRT display. When the program is called by the Monitor, it will respond by typing on the teletype

\title{
STREAMLINE GENERATION
}

and the overlay map assigned by the Monitor will be visible on the CRT display. The computer is now ready for directives to be input.

Directives

Pressing Button on Digitizer Cursor $\underline{\text { Task }}$

This locates the origin of the streamline. When the cursor button is pressed, a stream path is drawn on the CRT. When the computer is ready to respond for drawing a streamline, a message appears in the lower left corner of the CRT:

\section{DIGITIZE STREAMLINE ORIGIN}

During the actual calculation this message is not visible.

Sets direction of streamline from the input origin, whether the path is up or down gradient. It is simply a toggle switch, with the initial condition being up gradient. 
Directives

$\phi$

$\Omega$

$x$

$\alpha$

$+$

$\underline{\text { Special Keys }}$

CHAR ERASE $\underline{\text { Task }}$

Enter contour mode. When this button is pressed the program is set to draw contours and responds with a message in the lower left corner of the CRT:

ENTER CONTOUR VALUE

The contour value is then entered via the keys on the CRT console and input to the computer when the RETURN is pressed. The value is entered in scientific notation format $( \pm X . X X X X X \pm X X)$.

The switch is a simple toggle type with the initial setting not in the contour mode.

Clear the display of all streamlines and contours. It returns the display to the overlay map only.

This is a save function. The current display can be protected from erasure by the $x$ option. When this toggle switch, initially set so that the first time it is pressed it saves, is pressed whatever is on the display will not be erased. The next time it is pressed, the save protect option is removed.

When this is pressed the streamline generation is terminated and control is returned for further directives.

Exit the Streamline functional block and return to the Iterative Sequence Monitor.

Deletes everything entered from the CRT keyboard. The entries from the keyboard start all over again as if nothing had been entered. 
Errors Detected by Streamline

NEED TO

INITIALIZE

WITH MONITOR

FILE NOT FOUND
The Streamline function block was entered before being initialized by the Iterative sequence Monitor. This error returns user to the computer operating system monitor.

A data matrix assignment to use (MAP) of (PTl) was not made before entering the streamline function block. This error returns user to the Iterative Sequence Monitor.

TRAVEL TIME CALCULATIONS (TRAVPR), (TRAVTR)

This functional block calculates travel times along streamlines. The directives are identical to those for the streamline functional block, with the addition of several options. The constant permeability version and the variable transmissivity version have the same directives. The reumonic TRAVPR refers to the constant permeability travel time model, while the TRAVTR neumonic refers to the variable transmissivity travel time model.

When the program is called by the monitor, it responds by typing on the teletype either

TRAVEL TIME CALCULATION (PERMEABILITY)

or

TRAVEL TIME CALCULATION (TRANSMISSIVITY)

Directives

$\pi$
Task

Places a limit on the travel time. When the travel time of a flow path exceeds this limit, the calculation is terminated and the program is ready to start another calculation. When this button is initially pressed, the computer responds with a message in the lower left corner of the CRT

\section{ENTER LIMIT VALUE}

The limit is input with the display keyboard in scientific notation format and terminated with RETURN. When the value has been input the message 
Directives

$\pi$ (continued) on the CRT changes back to $\lambda$
Put the program in step mode. At the end of each iteration the computer waits in an idle loop until released. This is a toggle switch which is initial- ly in the normal free run form. In step mode, the program is released to do one more iteration each time this button is pushed.

Special Keys

These are identical to those of the streamline program.

Errors Detected by Travel Time

These are the same as in the Streamline program.

TRANSMISSIVITY CALCULATIONS (FLOWSS), (FLOWTR)

The Transmissivity Calculations prepare for and calculate a transmissivity distribution for a groundwater system. All of the directives for this functional block are entered from the graphic digitizer and CRT display; they are the same for both the steadystate (FLOWSS) and transient (FLOWTR) models.

When the Iterative Monitor first calls the transmissivity functional block the computer responds by typing on the teletype either

$$
\text { TRANSMISSIVITY CALCULATION (STEADY STATE) }
$$

or

TRANSMISSIVITY CALCULATION ( TRANSIENT)

Then a message appears in the lower left corner of the CRT

DIGITIZE FLOW TUBE ORIGIN 
The program is now ready to accept directives from the digitizer, CRT keyboard or CRT light pen.

Directives

DIGITIZER (Pressing Button on Digitizer Cursor) $\underline{\text { Task }}$

This locates the origin of the flow tube. At this time the message in the lower left corner of the CRT changes to

\section{ENTER TRANSMISSIVITY VALUE}

The user is to enter the transmissivity value at that point. This value is used as the initial transmissivity value of the flow tube. It is entered via the CRT keyboard in scientific notation and terminated with a RETURN ( $\pm X . X X X X X \pm X X)$.

During the flow tube calculation the message in the lower left corner of the CRT is not visible. When the computer is ready to do another tube the message

\section{DIGITIZE FLOW TUBE DRIGIN}

will again appear.

\section{CRT Keyboard}

$\Omega$

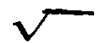

Remove all flow tube paths from display.

Change the direction of travel of the flow tube up or down the gradient (the initial direction is up the gradient). This is a toggle switch.

Exit the flow tube program back to the Iterative Monitor.

Abort the current calculation and return to the point to accept the start of another flow tube.

Move the tracking cross to the lower-left corner of the CRT display. 
Directives

$\uparrow$

$\leftarrow$

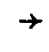

$\downarrow$

1

2

3

DELETE LINE

INSERT LINE

ERASE DISPLAY

]

[

SOM $\nabla$ $\underline{\text { Task }}$

Move the tracking cross vertically upward.

Move the tracking cross horizontally to the left.

Move the tracking cross horizontally to the right.

Move the tracking cross vertically downward.

Set the step size of the tracking cross to 1 unit.

Set the step size of the tracking cross to 10 units.

Set the step size of the tracking cross to 100 units.

Set the center of the tracking cross to the end point from which to generate the reference line.

Draw reference line from the initial point to the center of the tracking cross.

Remove the reference line from the CRT display.

Enter the line detect mode. This means that any flow tube that intersects the reference line will be terminated there and its transmissivity value and coordinate will be stored for generating the transmissivity distribution along the reference line.

Exit the line detect mode.

Change the size of the tracking cross. This is a toggle switch which changes the size of the tracking cross from small to large and vice versa. The small cross is for drawing curves on the CRT, the large one is for locating points. 
Directives

s

ERASE LINE

$-$

$\bowtie$

TRACKING CROSS

(Large)

TRACKING CROSS

(Small)

LIGHT PEN

\ SET UP NEW LINE

$\bigotimes$ DISPLAY CHART
Task

Enter the draw mode. The curve entered into the computer by this key is defined by the curve drawn with the light pen and tracking cross. This curve is made up of segments of equal length in the horizontal direction.

Erase the last segment of the curve being entered.

Set up to draw the box defining the eligible modes for generation of the transmissivity. The first time this button is pressed a pair of hairlines appears on the CRT which can be transferred to any point with the tracking cross and light pen. Once the pair of lines has been located, the next pressing of the (-) switch locks that point as one of the corners of the box determining the region of eligible points. The box is now drawn by moving the tracking cross.

Set the box defining the region of eligible points for which the transmissivities are to be calculated.

Used to locate points on the CRT. These points can serve various purposes, depending on the keys pressed.

Draws curves made up of segments with a constant horizontal length which are entered into the computer.

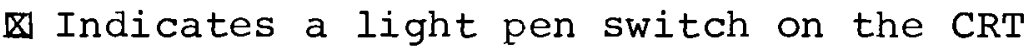
display screen.

Graph the data of transmissivities along the reference line for the first time.

Display the graph of transmissivity along the reference line after the graph has been formed. 
$\underline{\text { Directives }}$

\CALCULATE T

$\triangle$ FORM FUNCTION

TABLE

\ RETURN

REF LINE AT 32000 FT

Special Keys

CHAR ERASE
Task

Start calculating the transmissivities for the modes within the eligible region and the reference line. This stores them in a data matrix.

The curve that has been entered by the tracking cross as the curve defining the actual transmissivity distribution along the reference line is stored for future retrieval.

Display the map of the system being analyzed.

A reference line longer than 32000 feet was drawn. This returns user to the start of the program, eliminating all previous actions.

Deletes everything just entered by the display keyboard. Additional typing on the keyboard will be input as if nothing had been previously entered.

Errors Detected by Transmissivity Calculation Program

INITIALIZE WITH ITERATIVE MONITOR This functional block has not been initialized with the Iterative Monitor. This error returns the user to the operating system Monitor.

FILE NOT FOUND A required data matrix was not assigned by the Iterative Monitor. User is returned to the control of the Iterative Monitor. 


\section{ACKNOWLEDGMENTS}

This research was sponsored by the Advanced Technology Development Section, Research and Development Department, Atlantic Richfield Hanford Company. The authors are grateful for the assistance and direction provided by Dr. M. D. Veatch, Staff Hydrologist; D. J. Brown, Staff Geologist; and R. A. Isaacson, Manager of the Advanced Technology Development Section, Atlantic Richfield Hanford Company.

The authors also appreciate the editorial assistance given by Leila Counts, and the help of Jan Greenwell, Velva Harris, and Loretta Howard in preparing the manuscripts. 
APPENDIX A

EXECUTIVE PROGRAM LISTING 


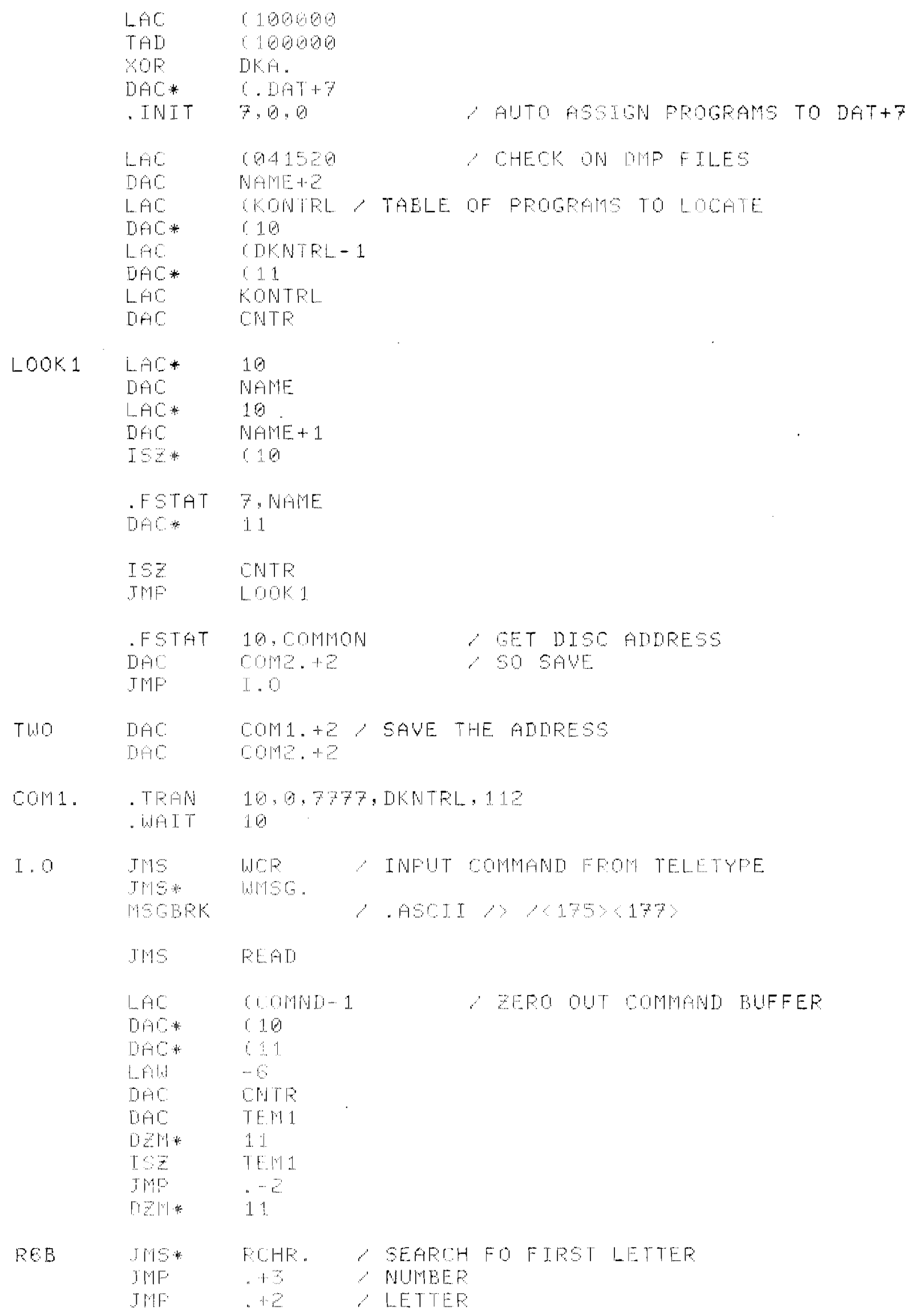




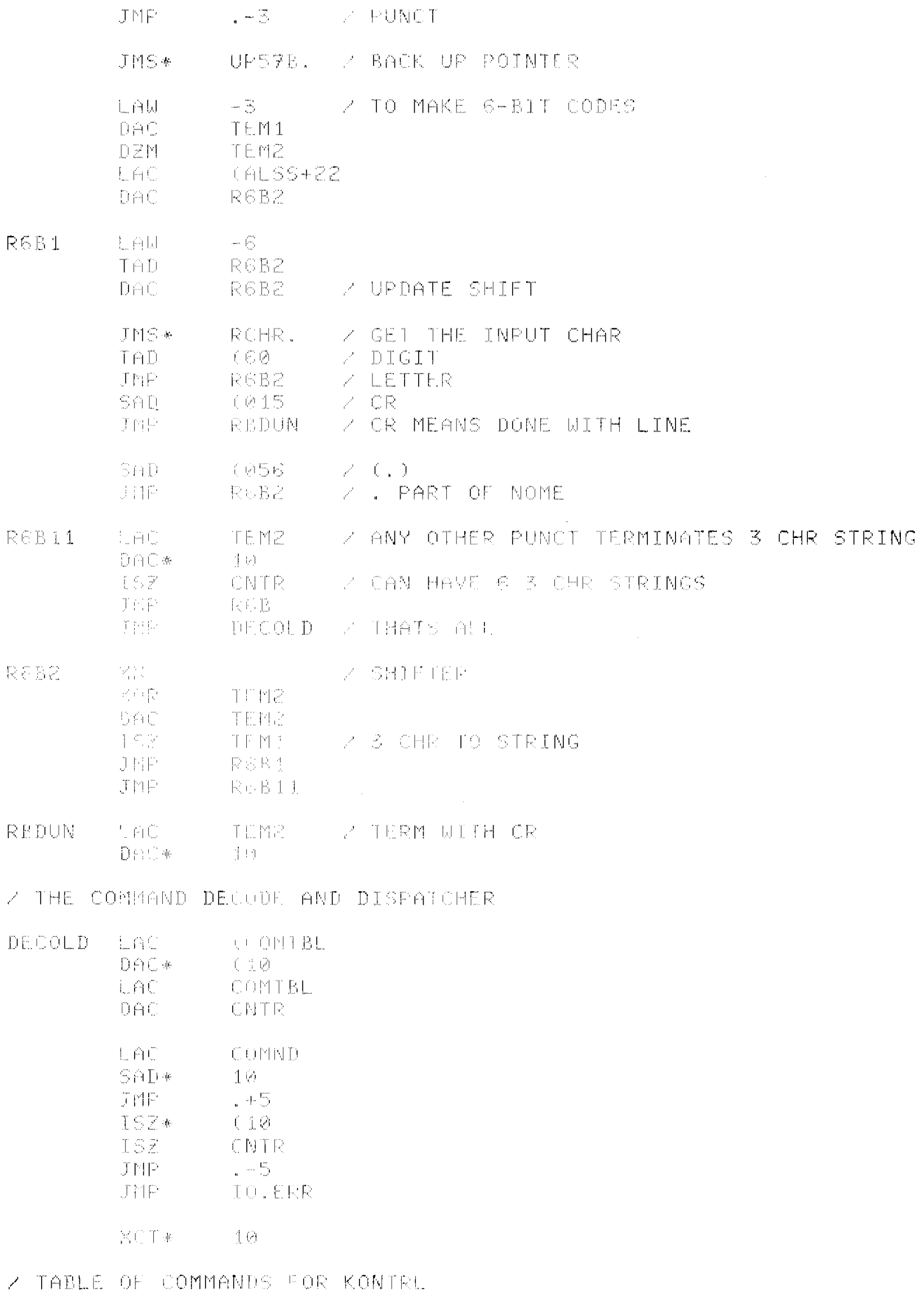




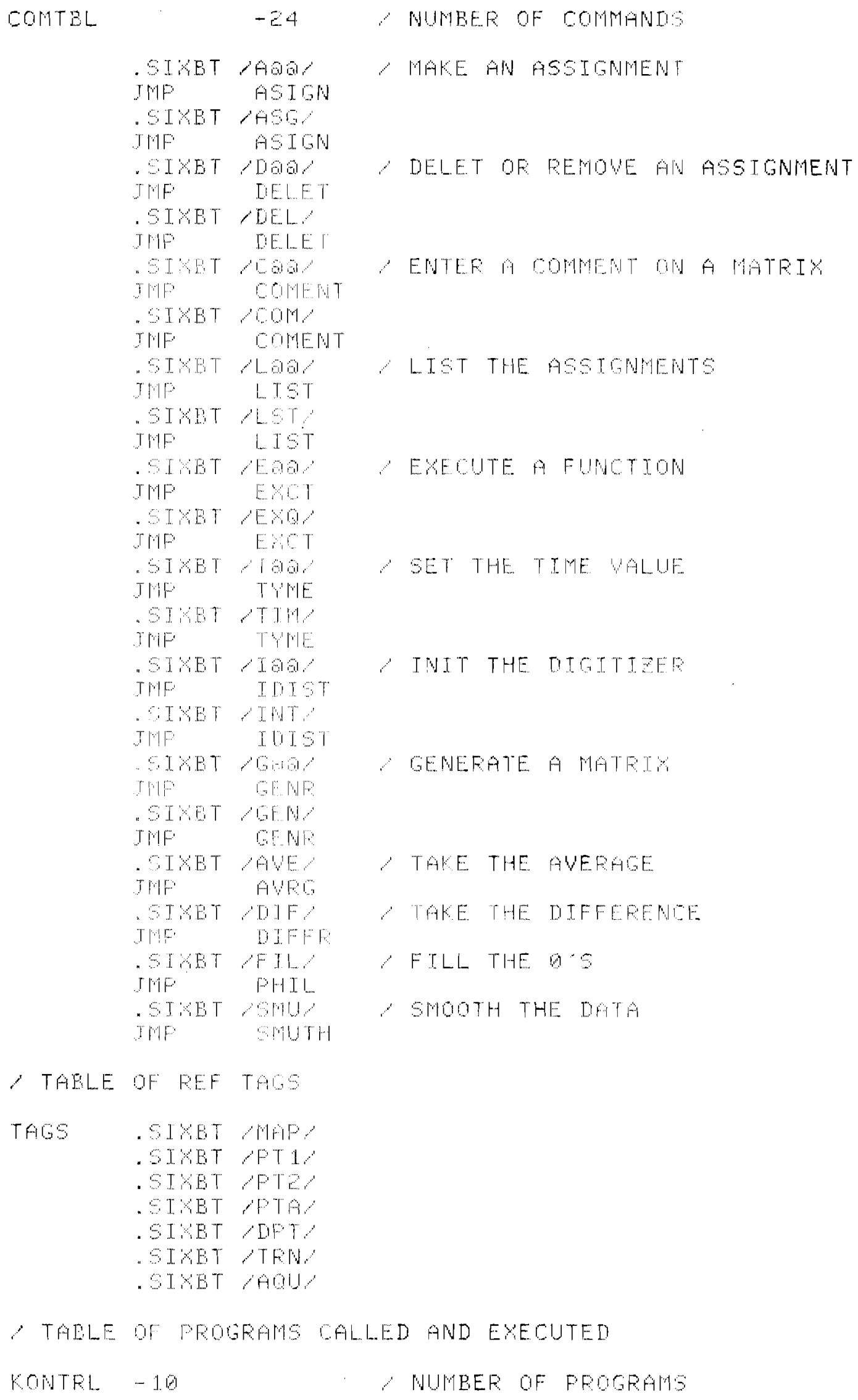




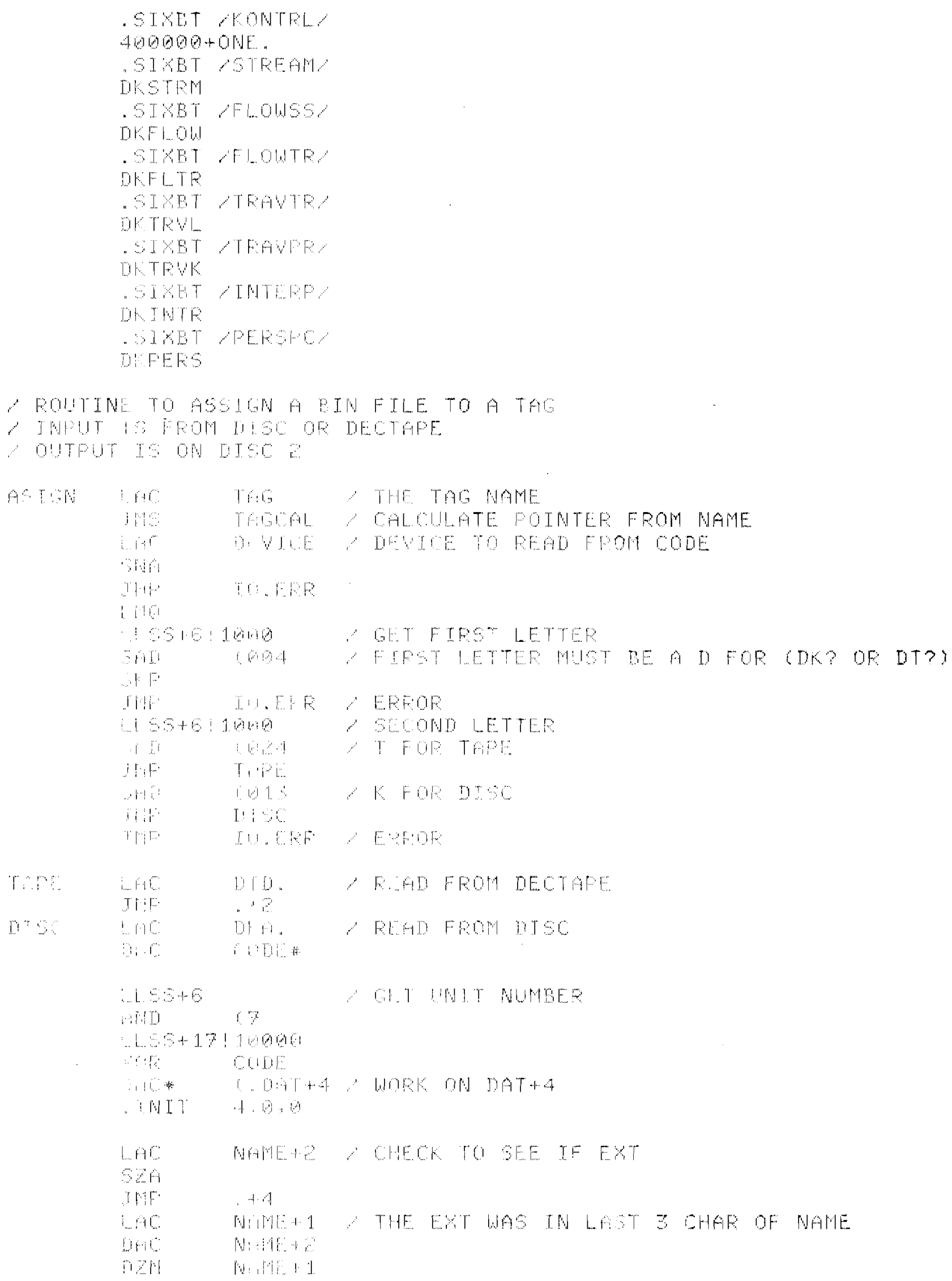




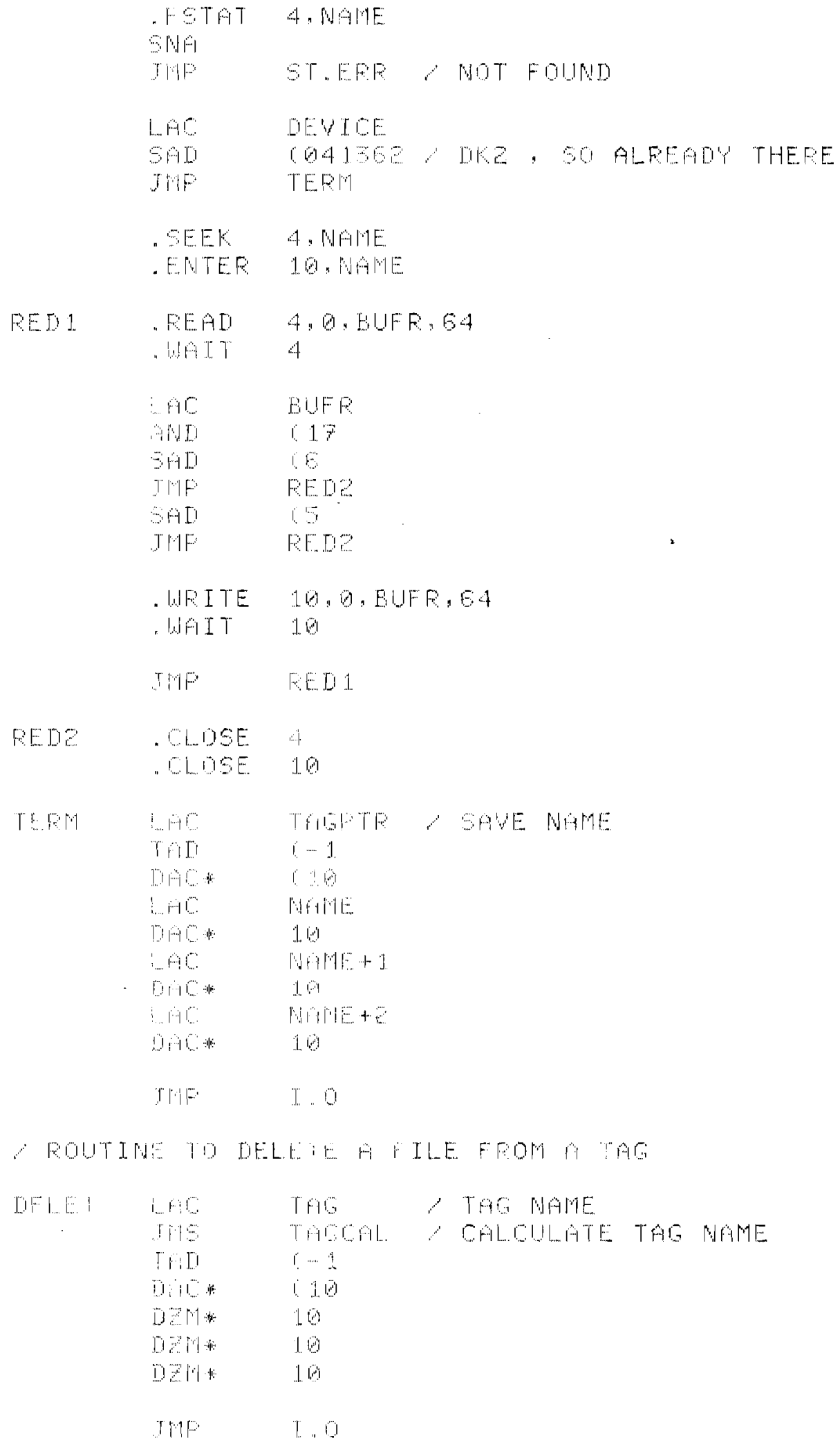




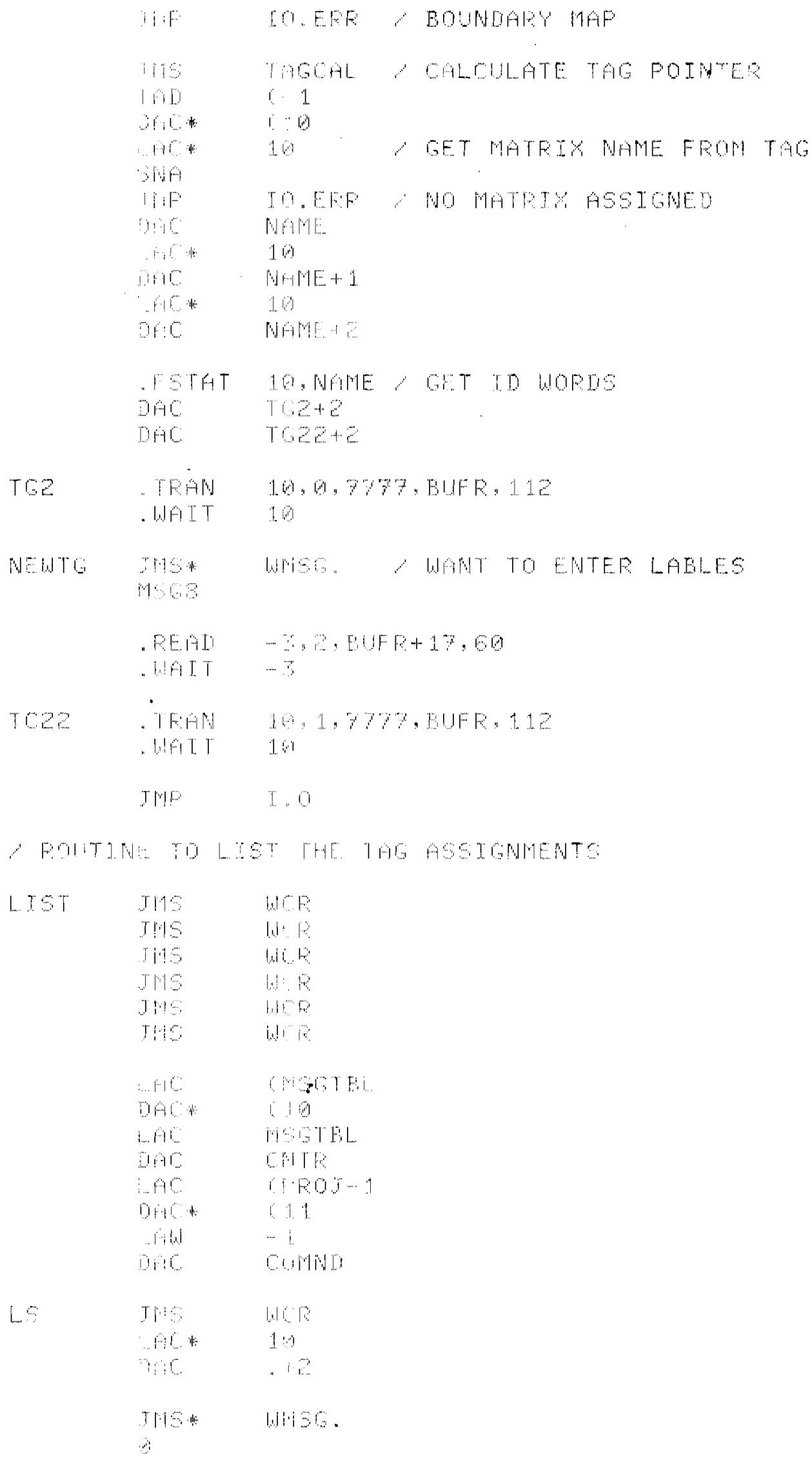




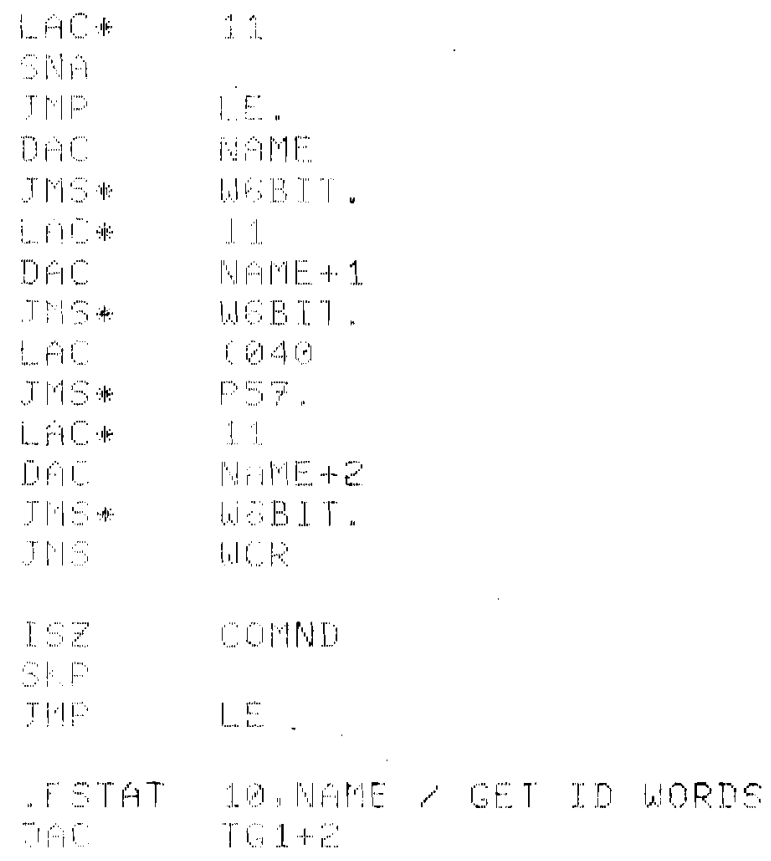




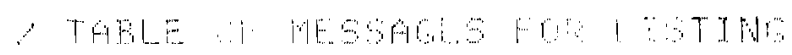

$195+36$

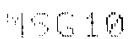

Ml 1

$1,1,1,19$

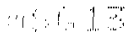

40.14

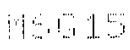

M: 1.6

Y WO TO EEOUTE A PFOCPM

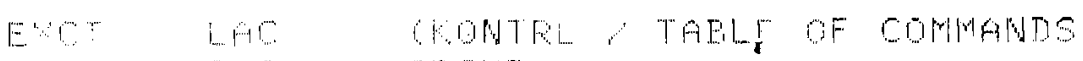

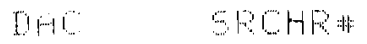

irition

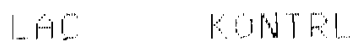

WHAR WUEER OF ENFTES TW TABLE

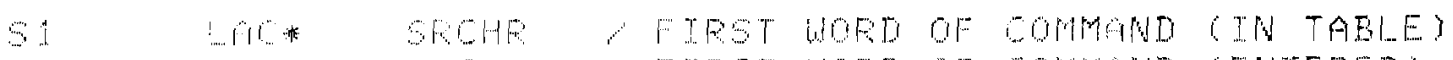

SID HO FIFST WOFD OF COWWHD IEWTERED

TWF FIFST PAFT WATEWE

I...

$31 P 1$

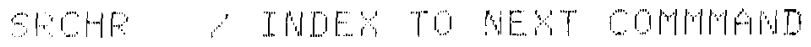

Mis $\quad$ का

-

TWE

TWE HOEF POE

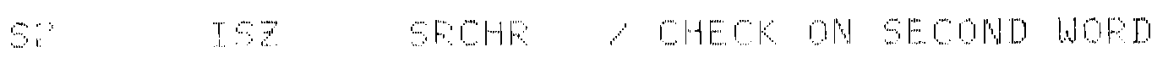

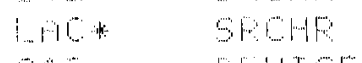

SHII DEVE

$19 \%$ " 3 MAOE A MीTCH

L HO

TiH $\quad S \ldots 1$

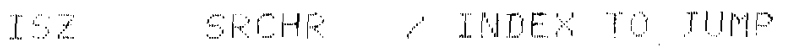

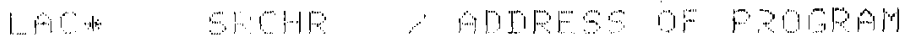

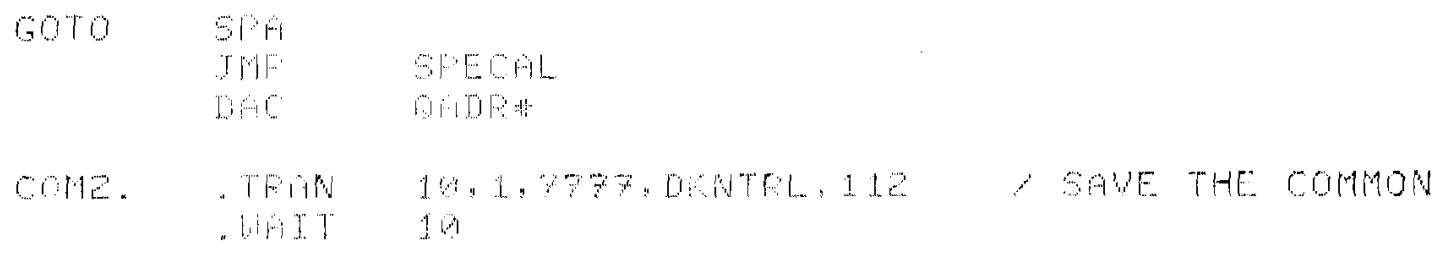

DICCA= $37 \%$

. Iद $1=37 \%$

. DKU1 $=37 \%$

- TKFUS $37 \% 3$

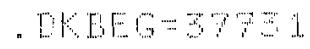

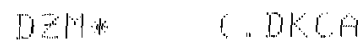

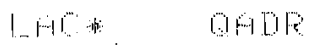

Sए। 


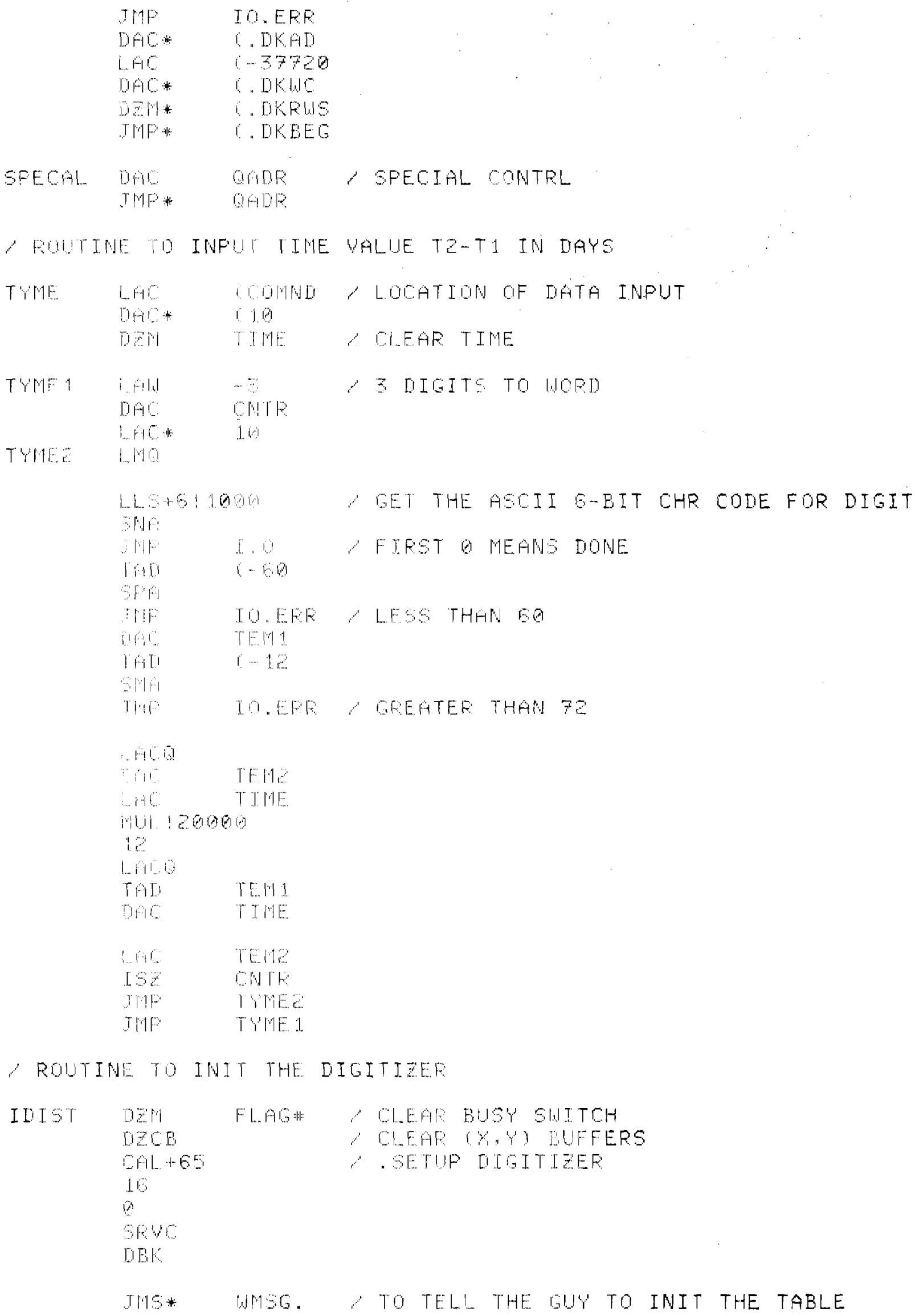




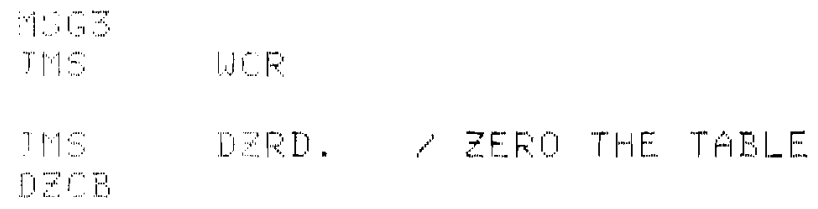




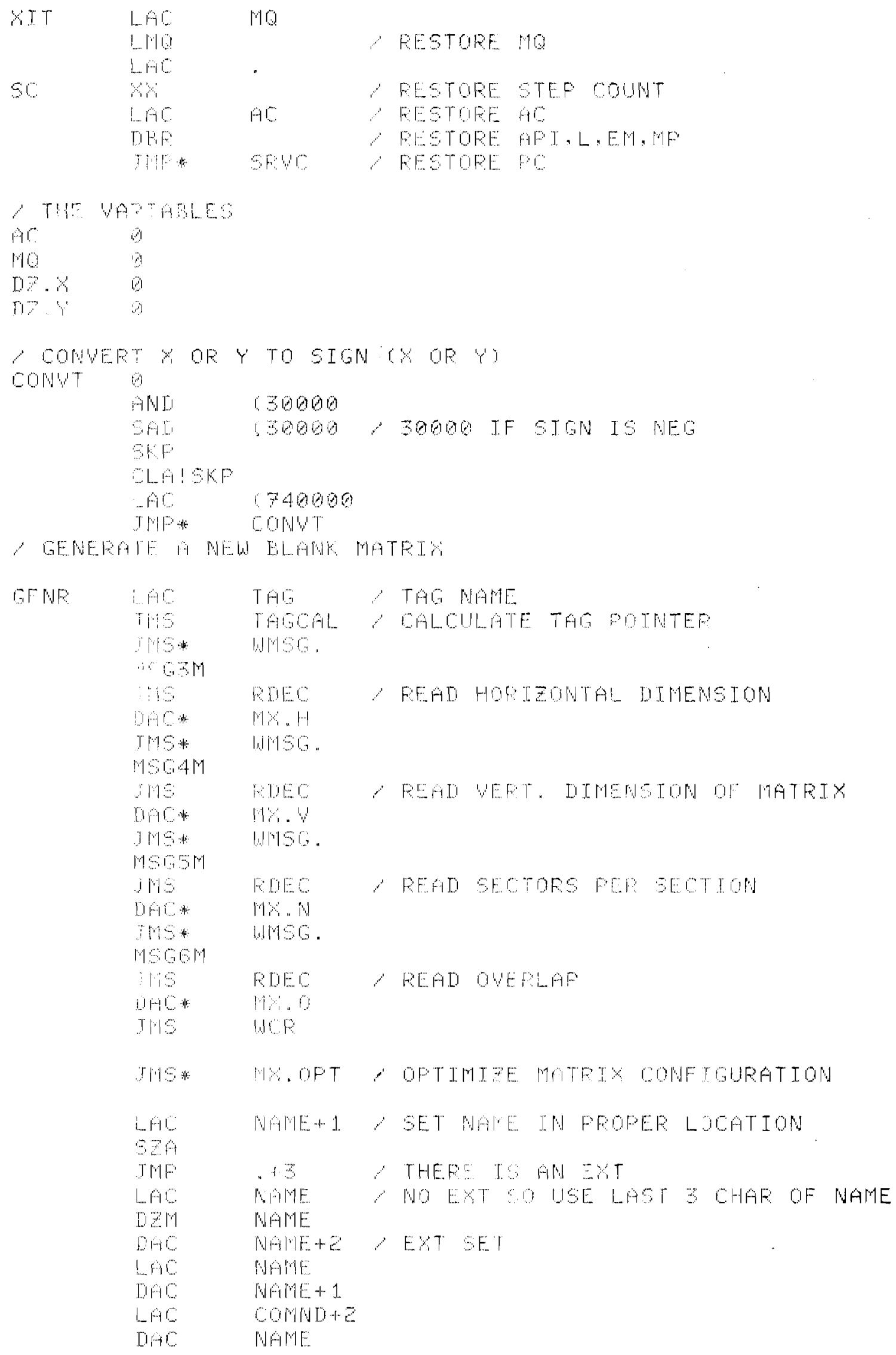




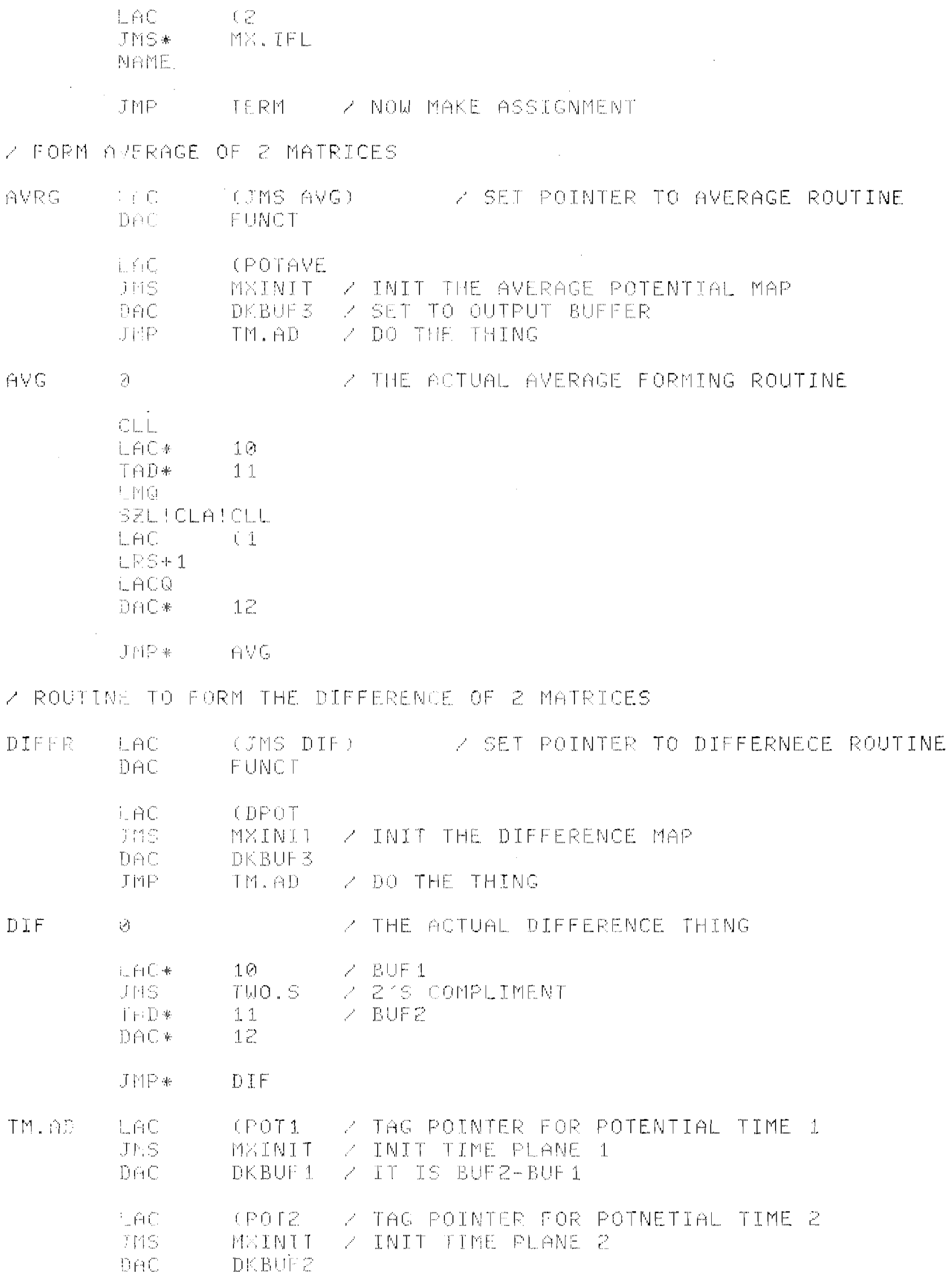




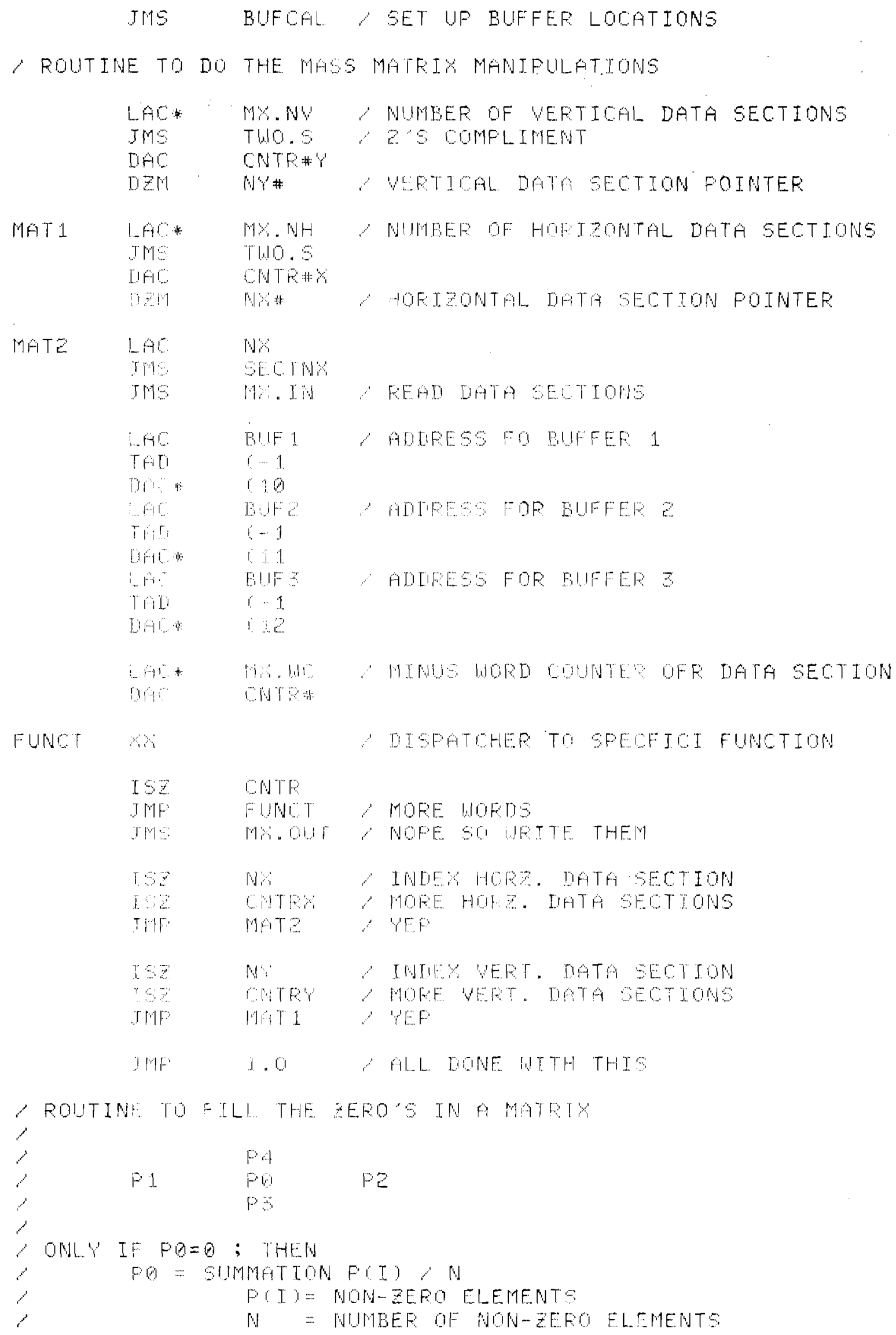




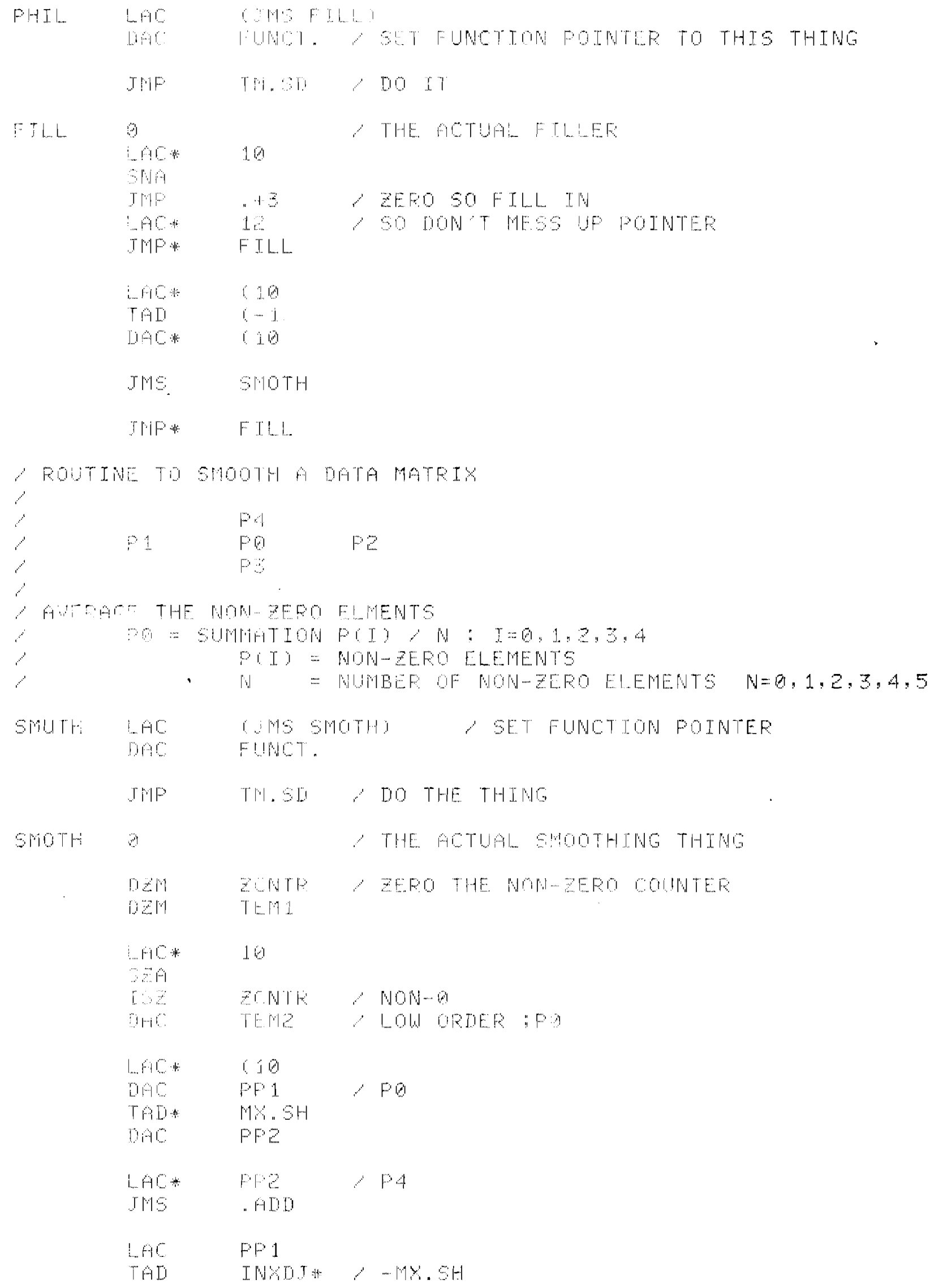




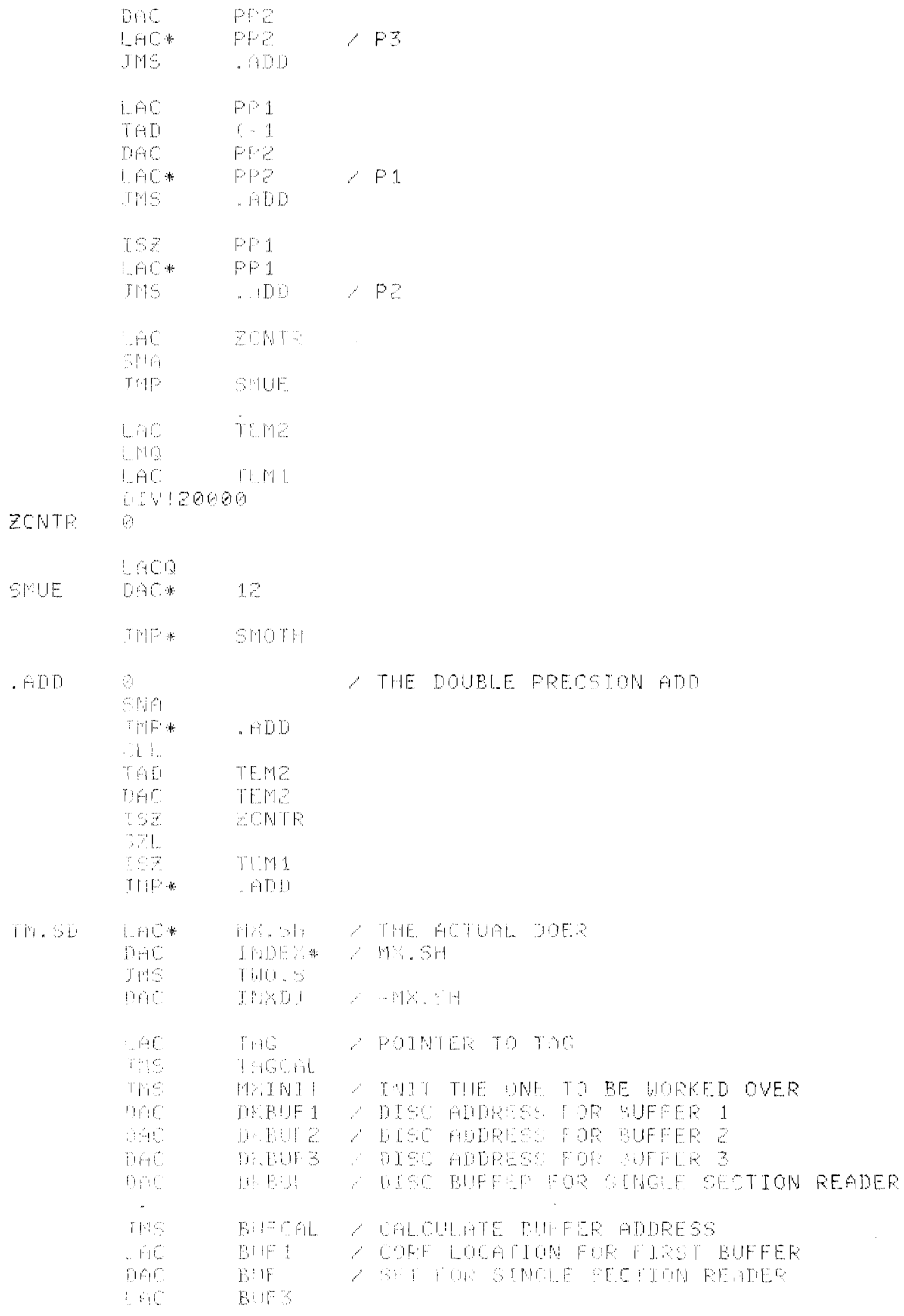

\section{$A-16$}




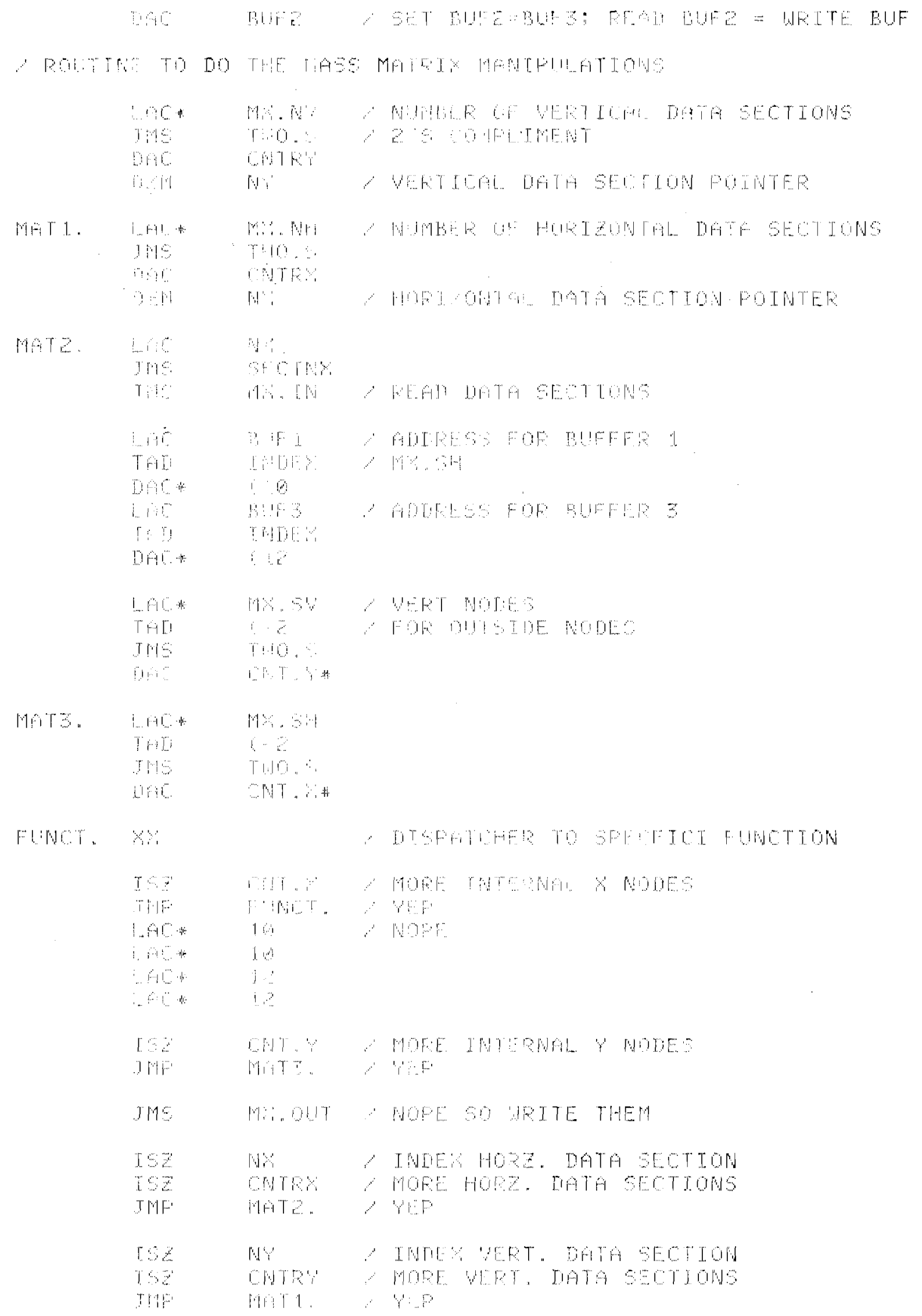




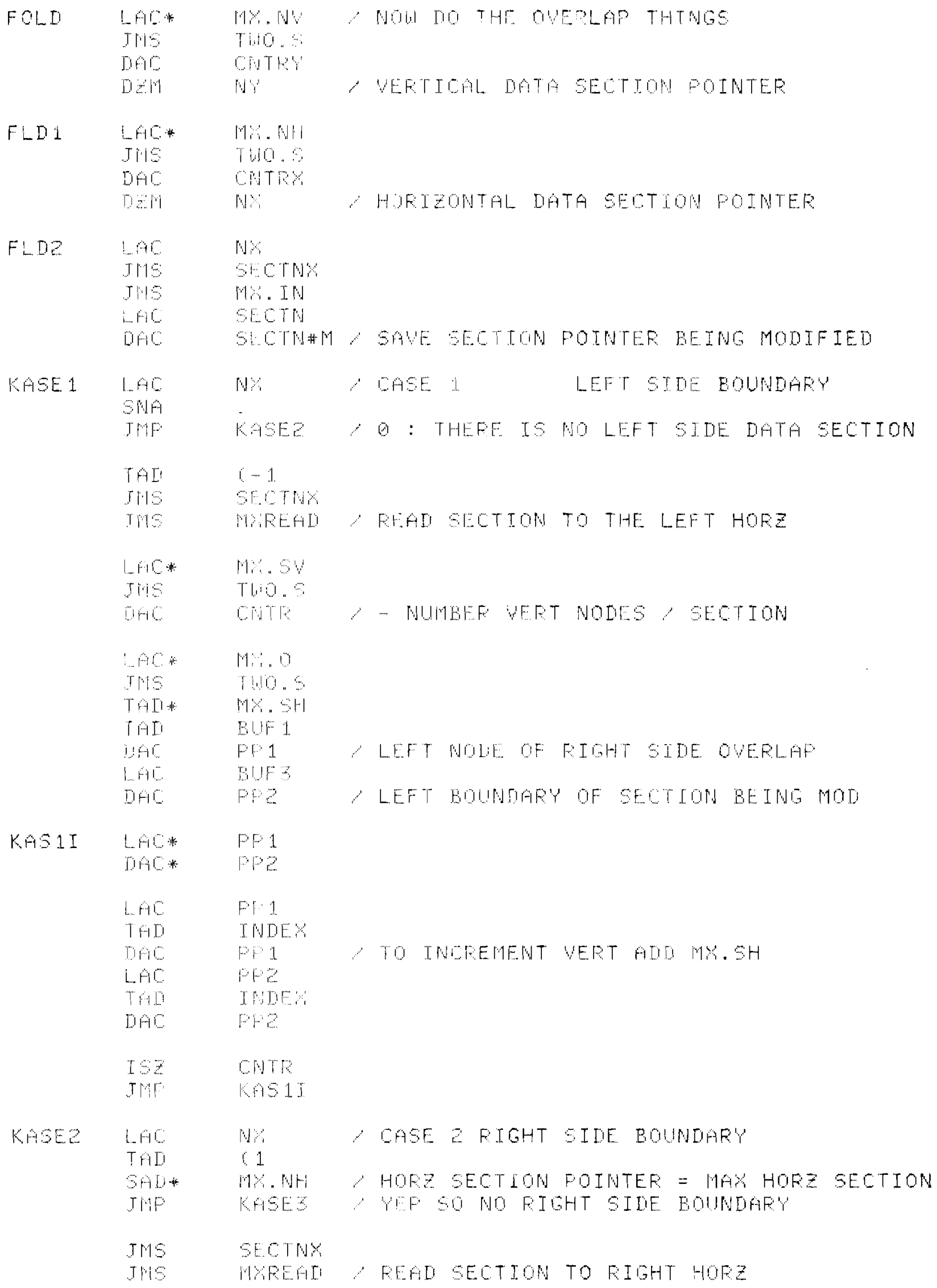




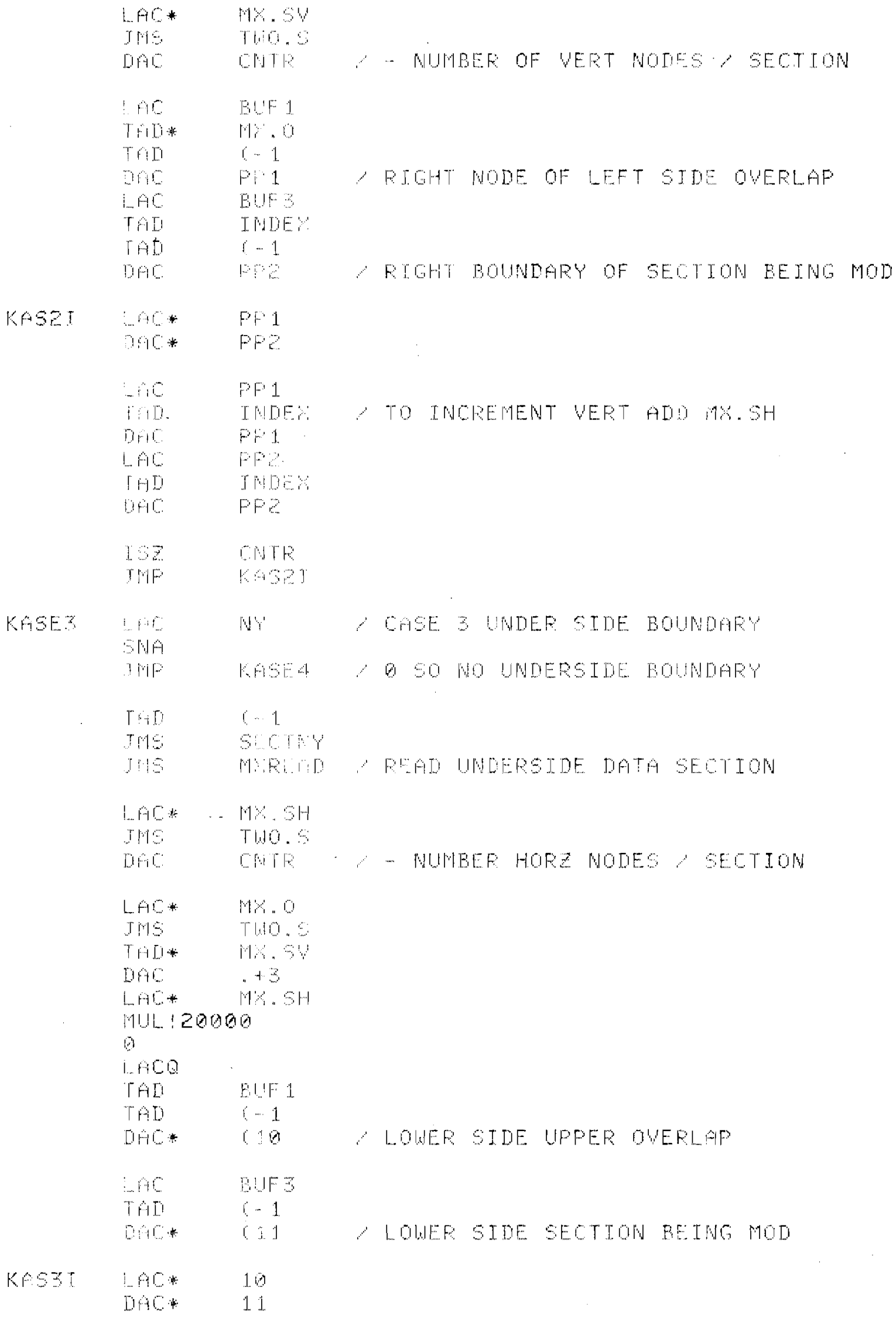




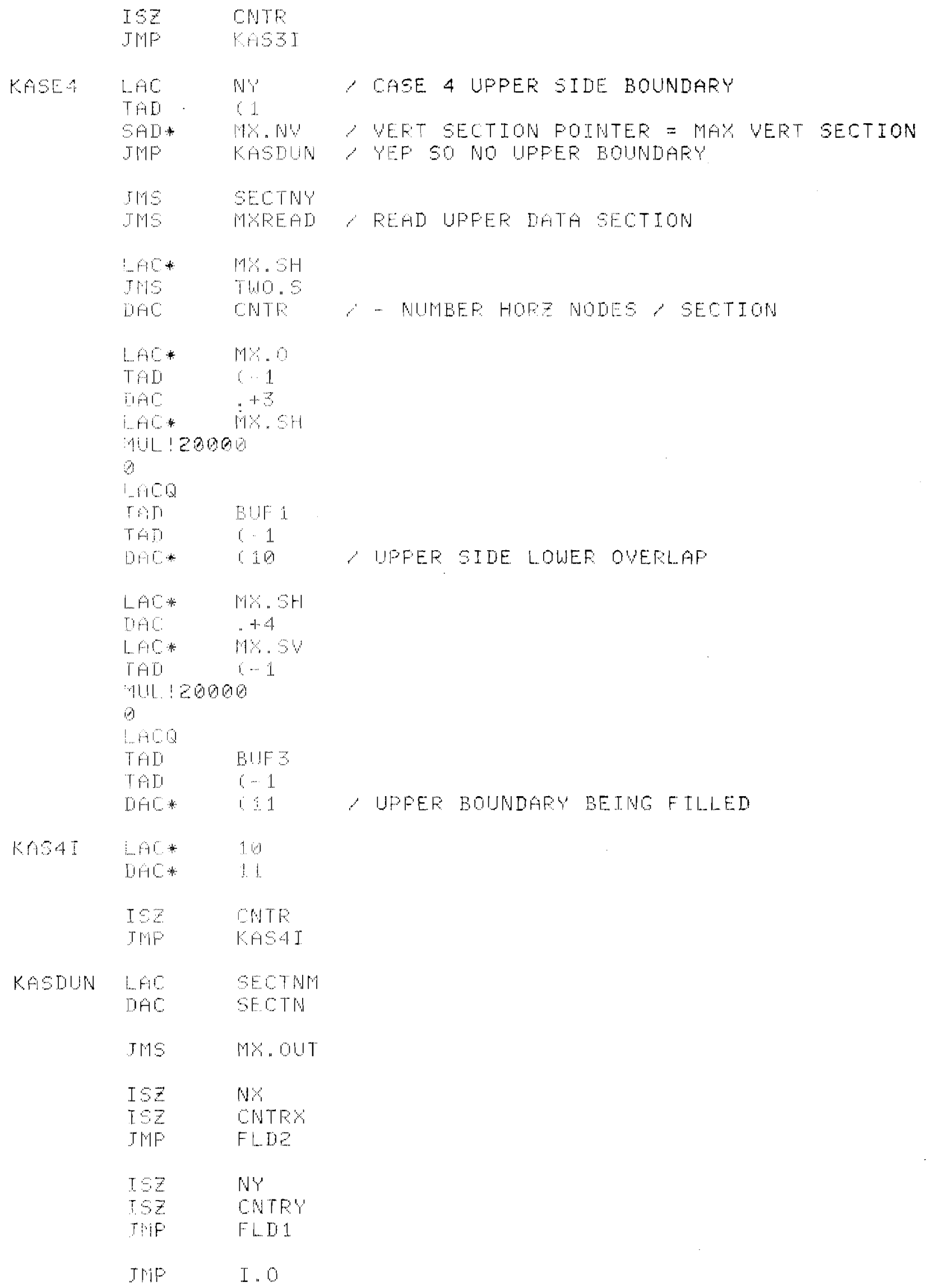


ERPORS

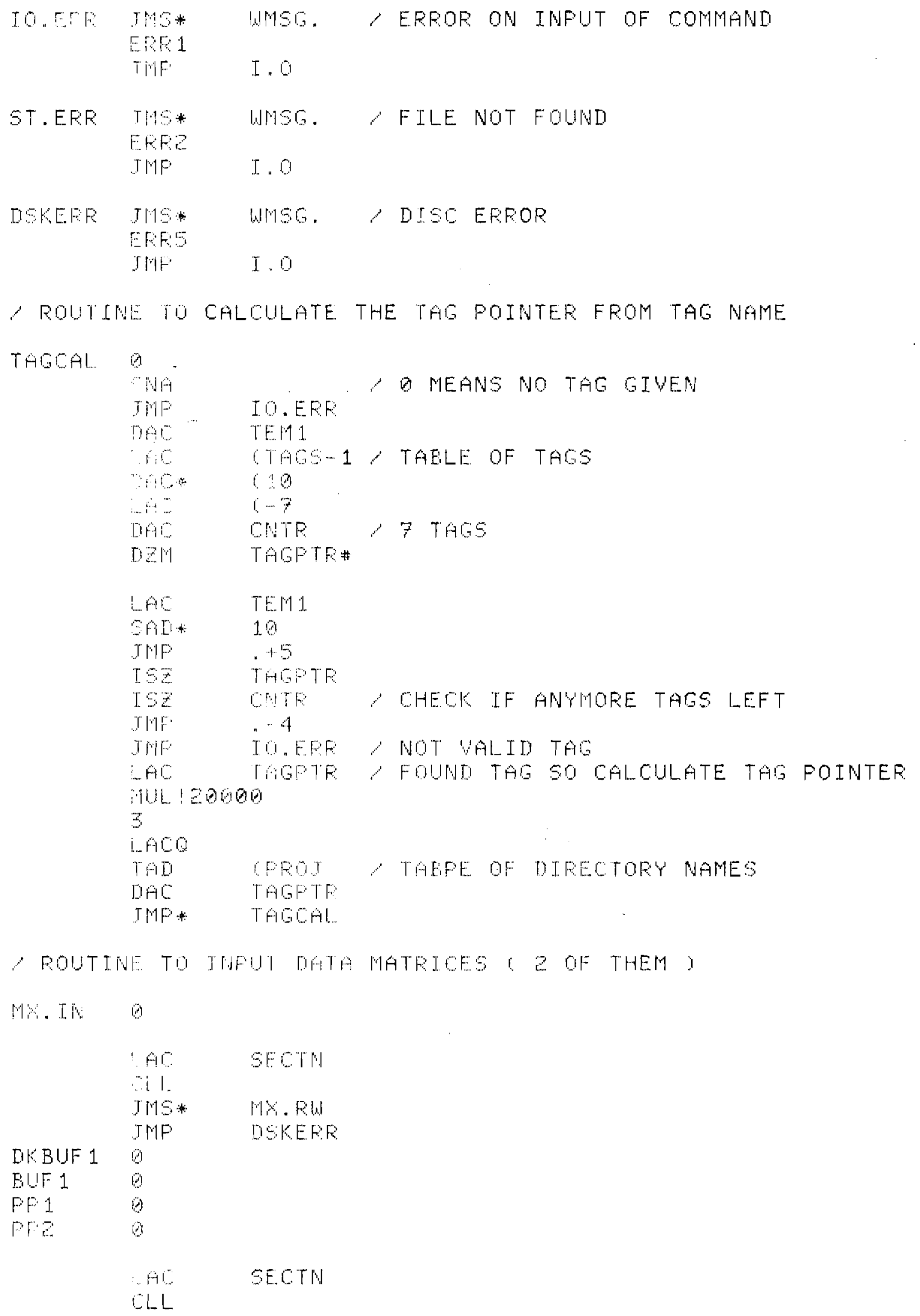




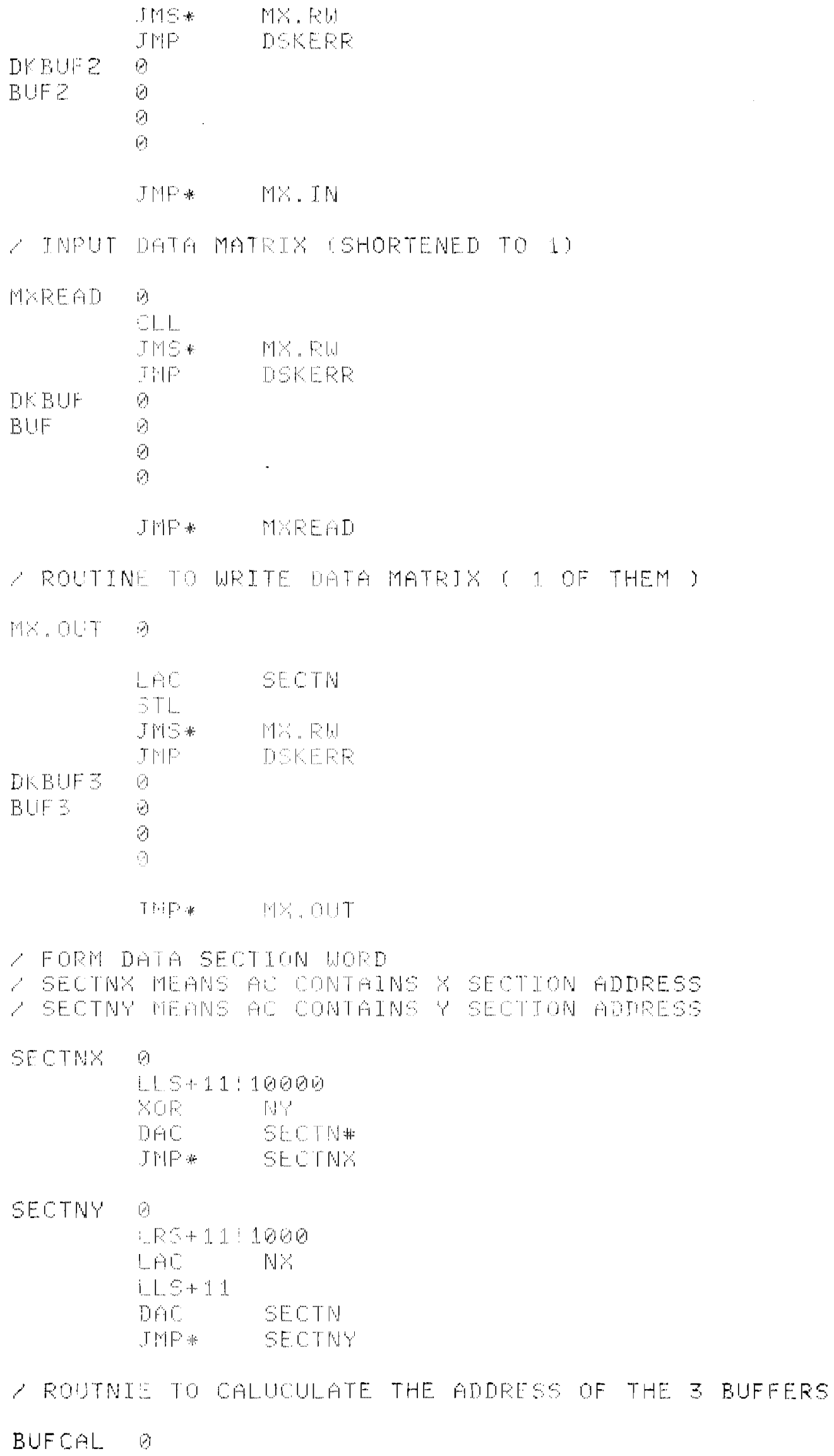




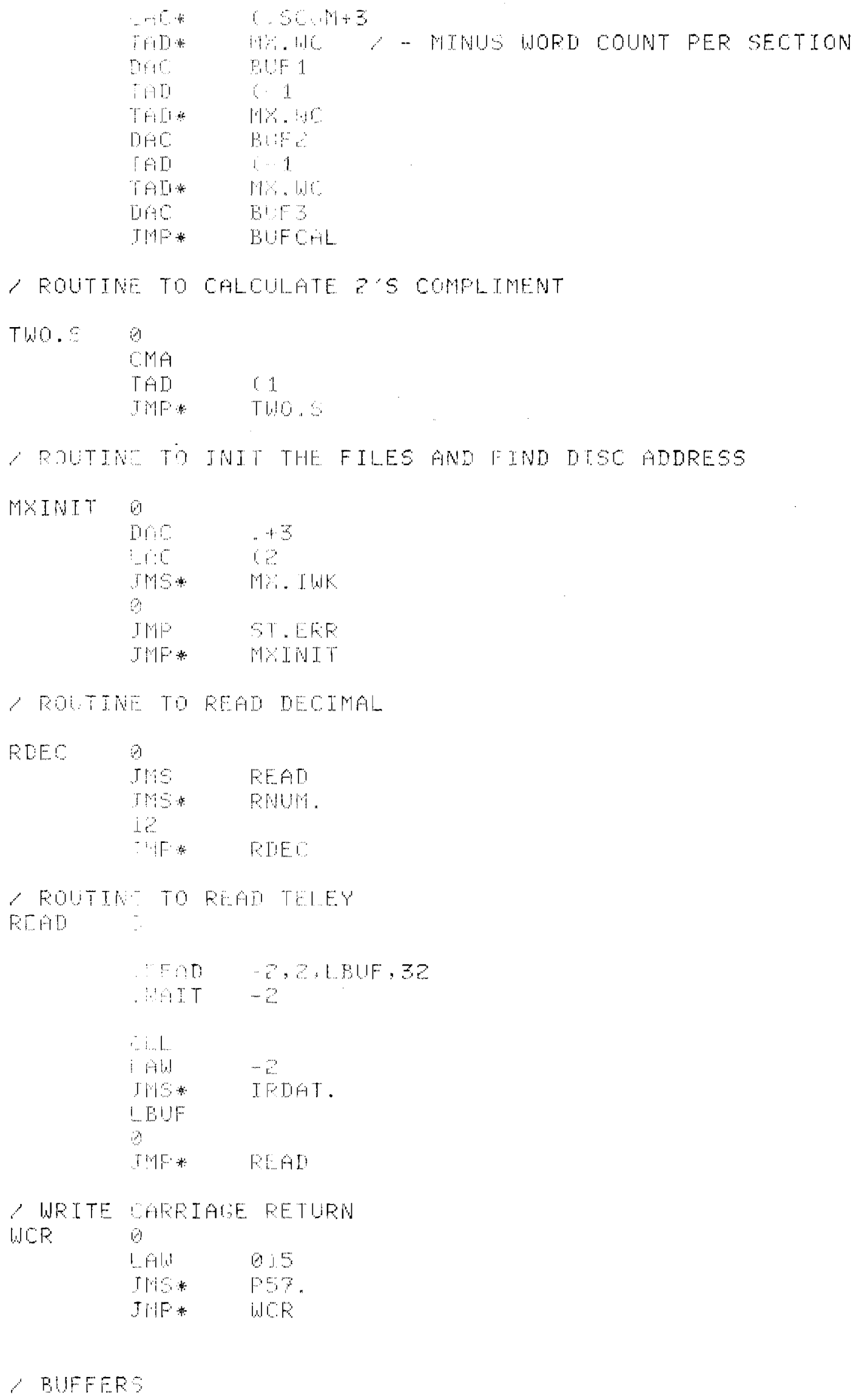




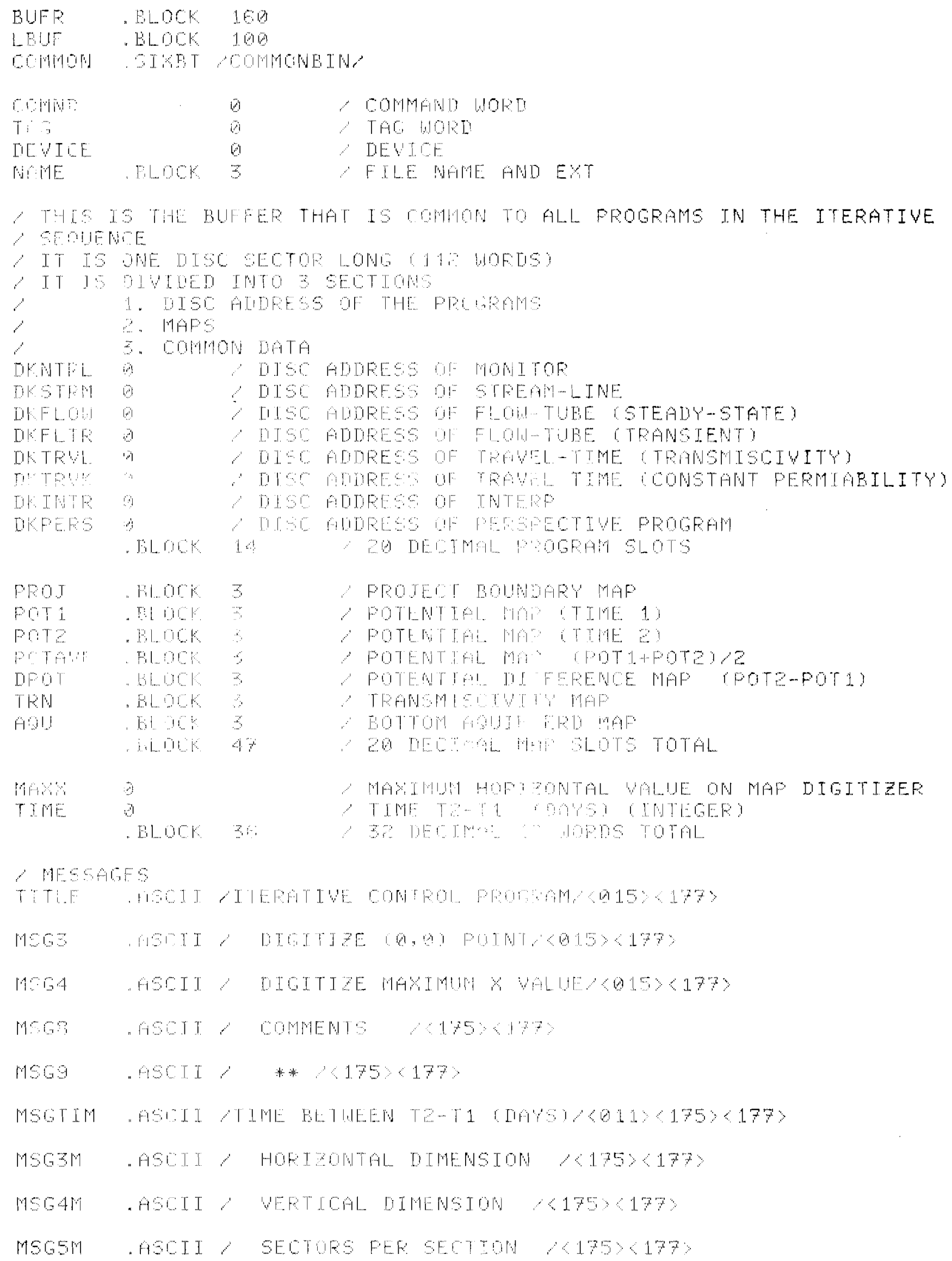




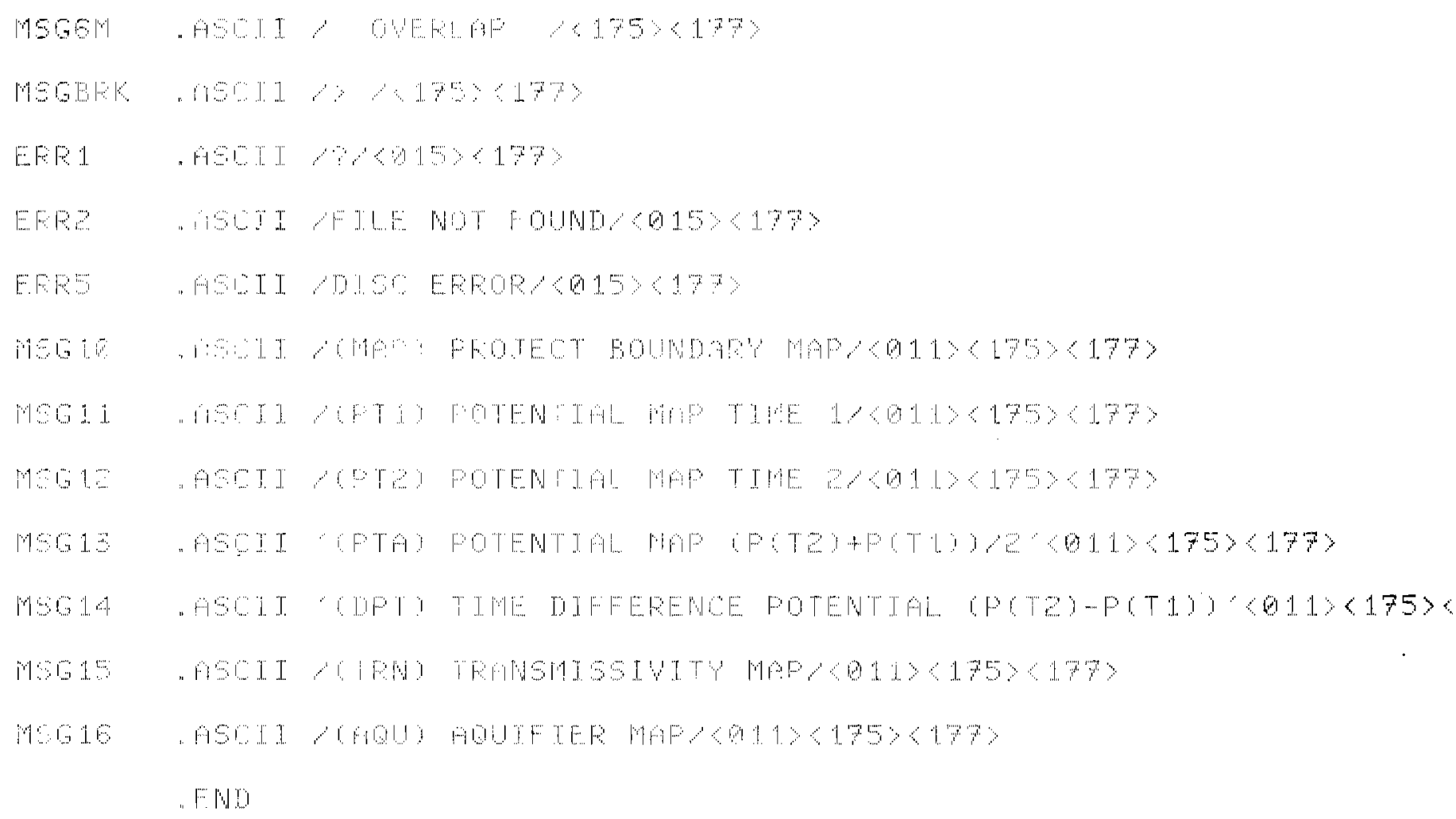


APPENDIX B

STREAMIINE PROGRAM LISTING 


\section{STREAMLINE PROGRAM}

-TITLE STREMMIME SET-IOP

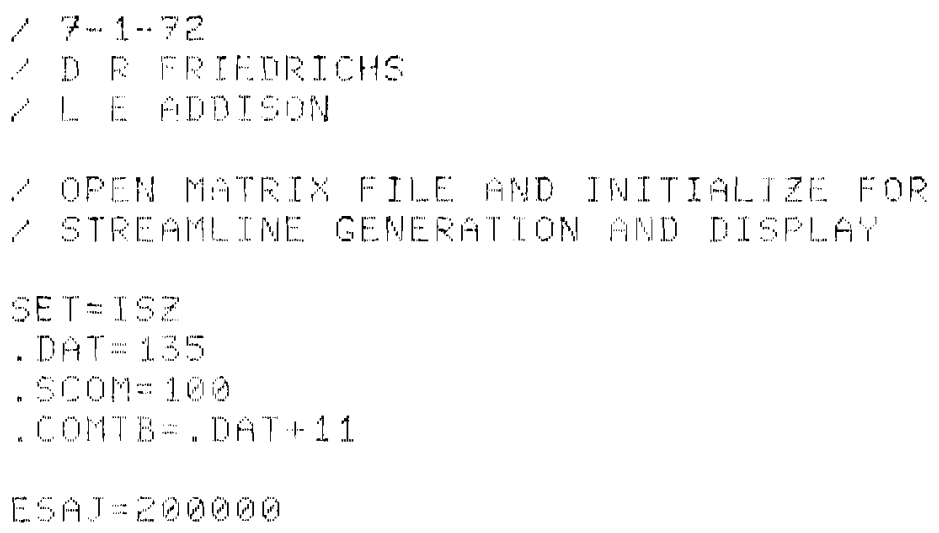




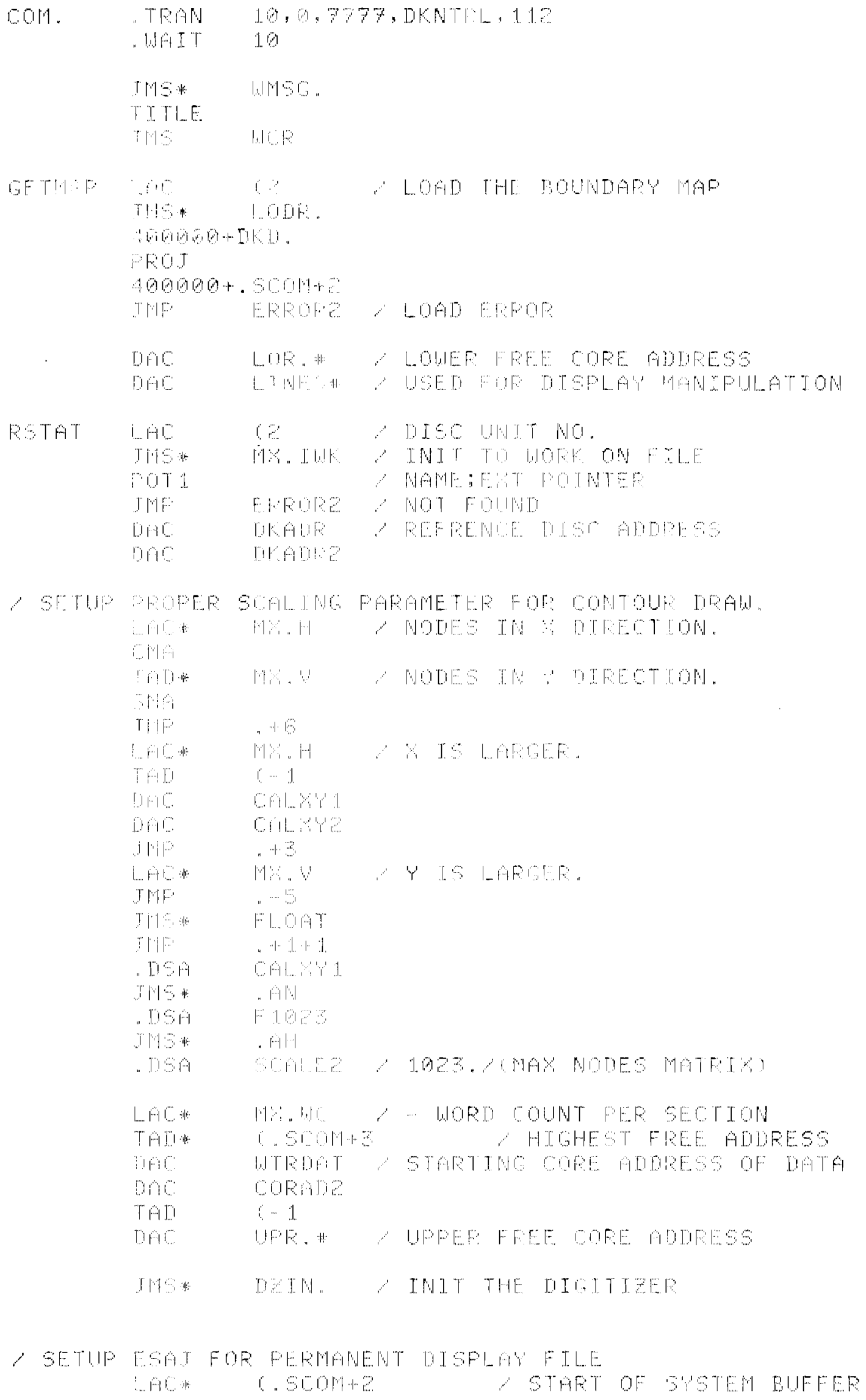




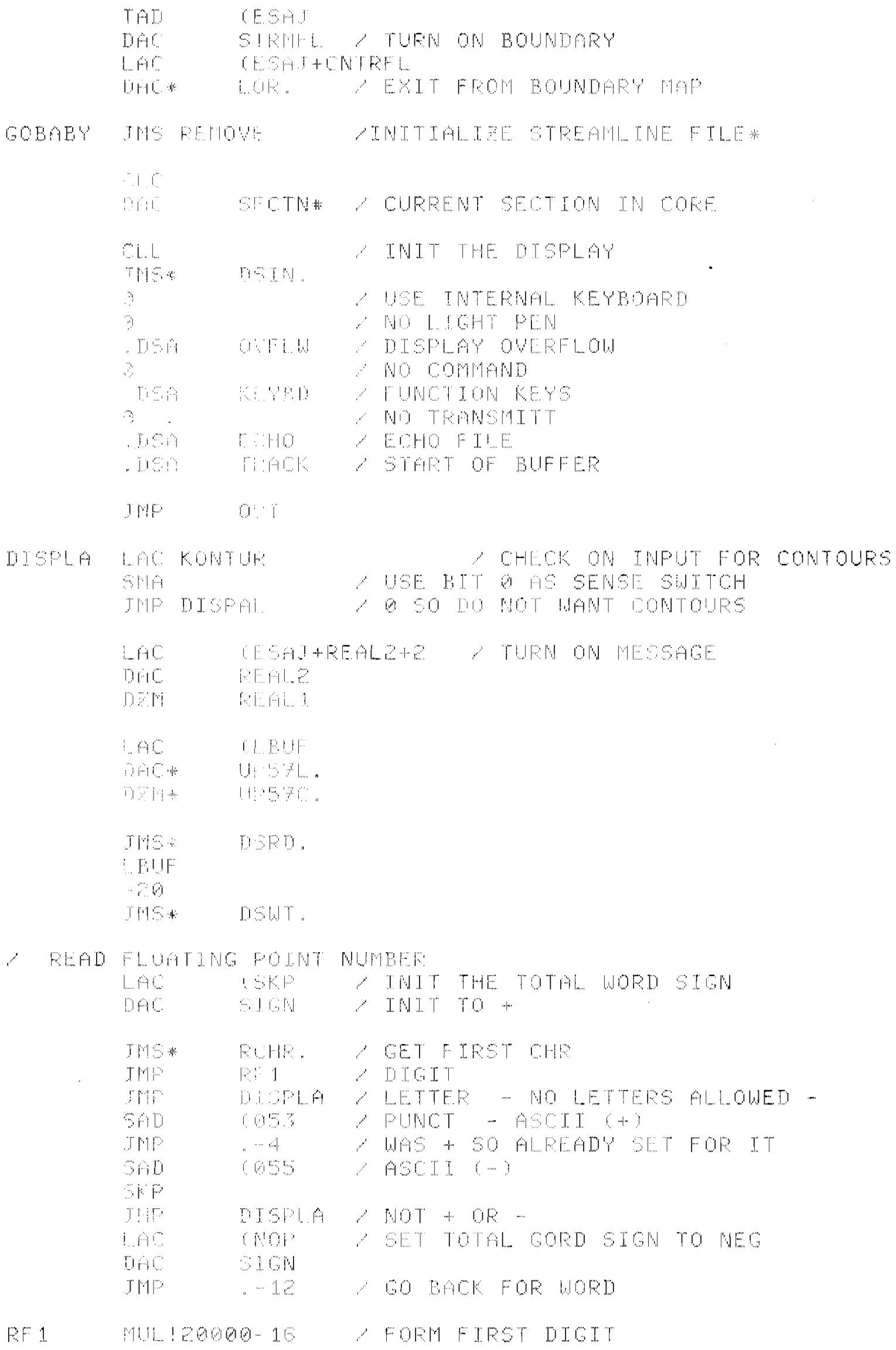




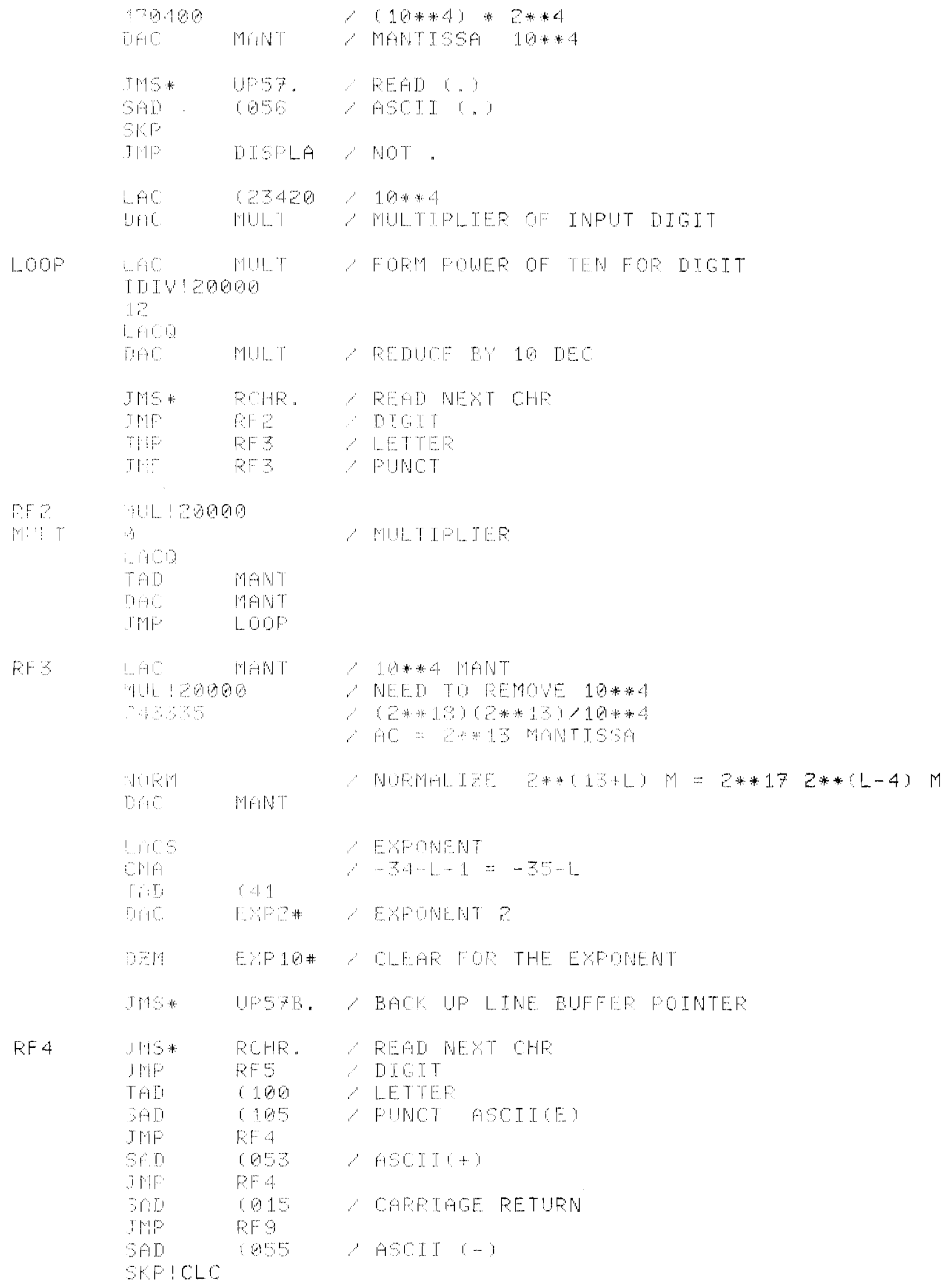




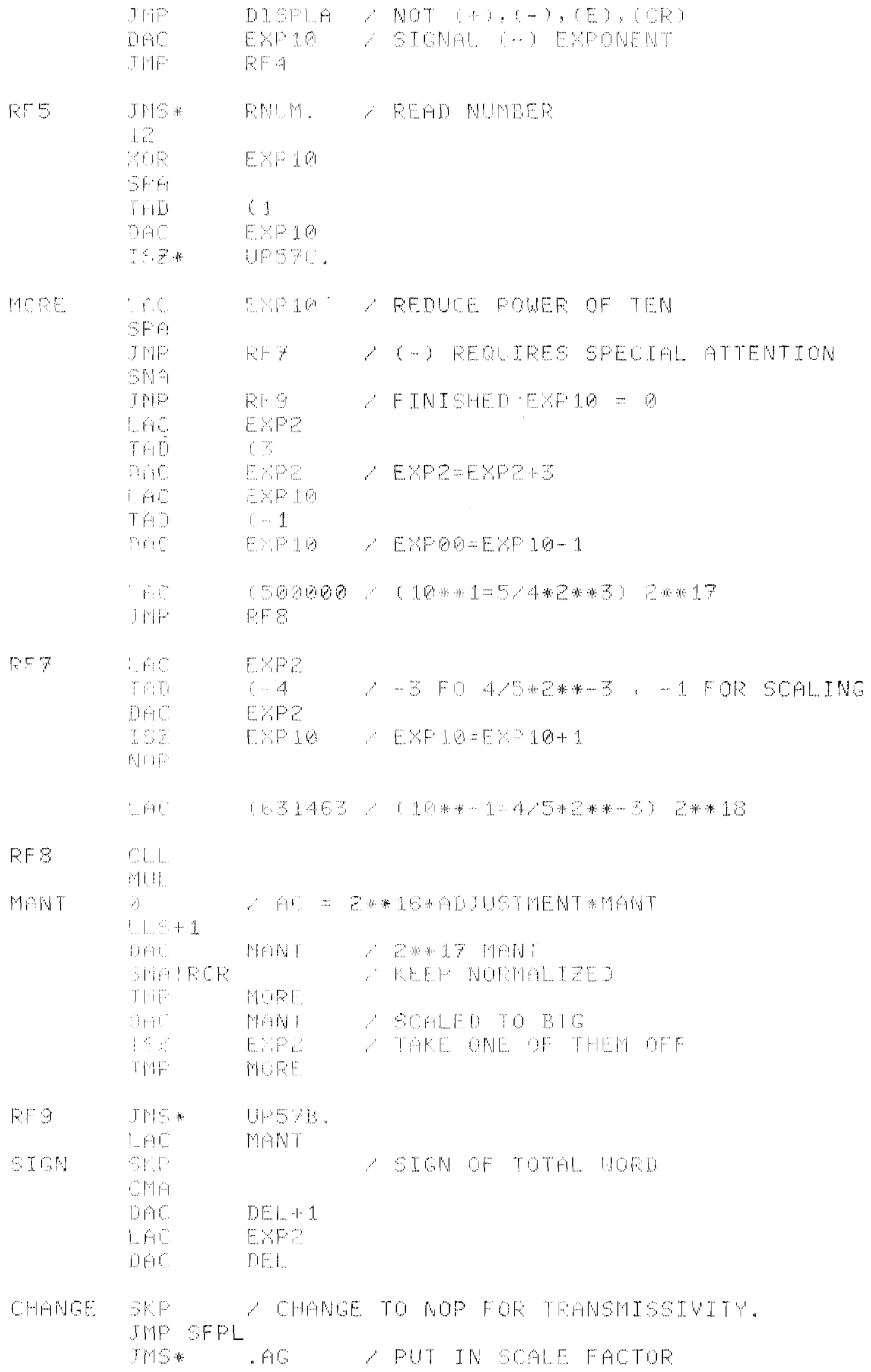




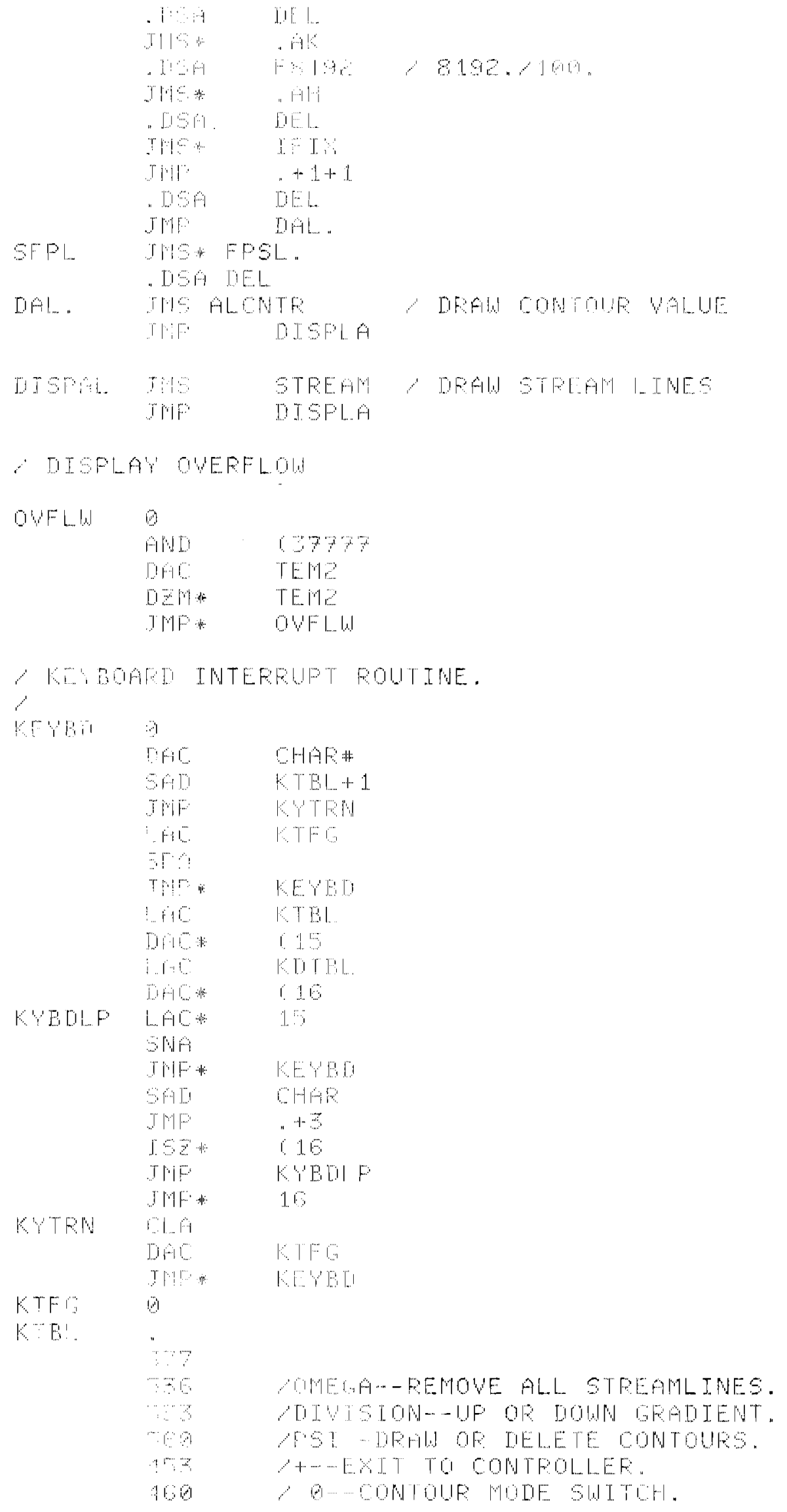




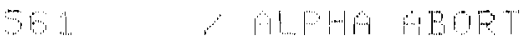

)

r

CTPL

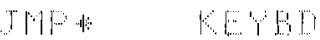

YEWOUE Al STEAM THES

TVIF

Y WAME GPATEDT"

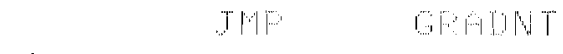

$r$

Exit TO CORTOLLF

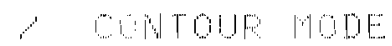

TPF - KOTF

ABOFT CHLEULTAOS

THP $\quad \mathrm{ABOT}$

is

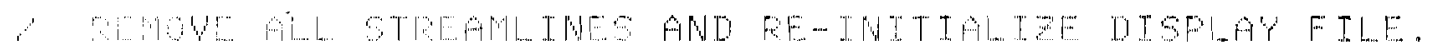

Ply

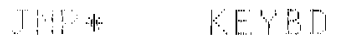

PEPOO

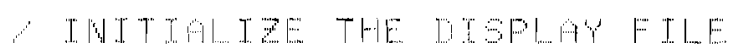

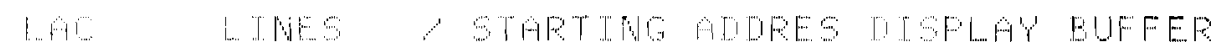

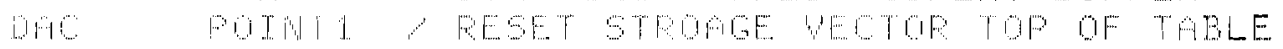

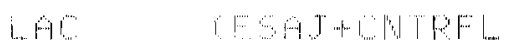

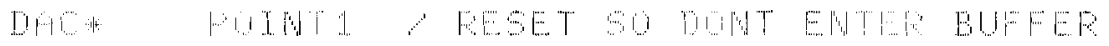

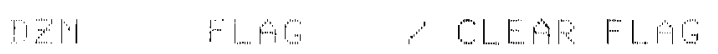

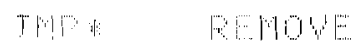

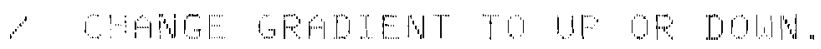

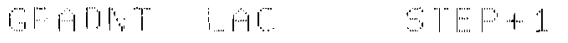

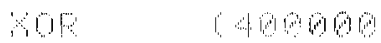

blin.

TI

$y \quad \mathrm{~T}$ -

X.. $\mathrm{CAF}$

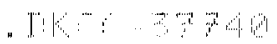

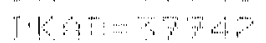

. 161

. THQ1S=8\%

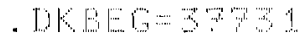

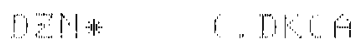

L...

$5 n$

Tline

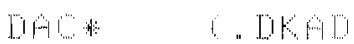

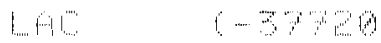

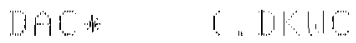

0Z1

TPH

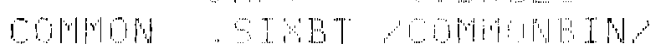




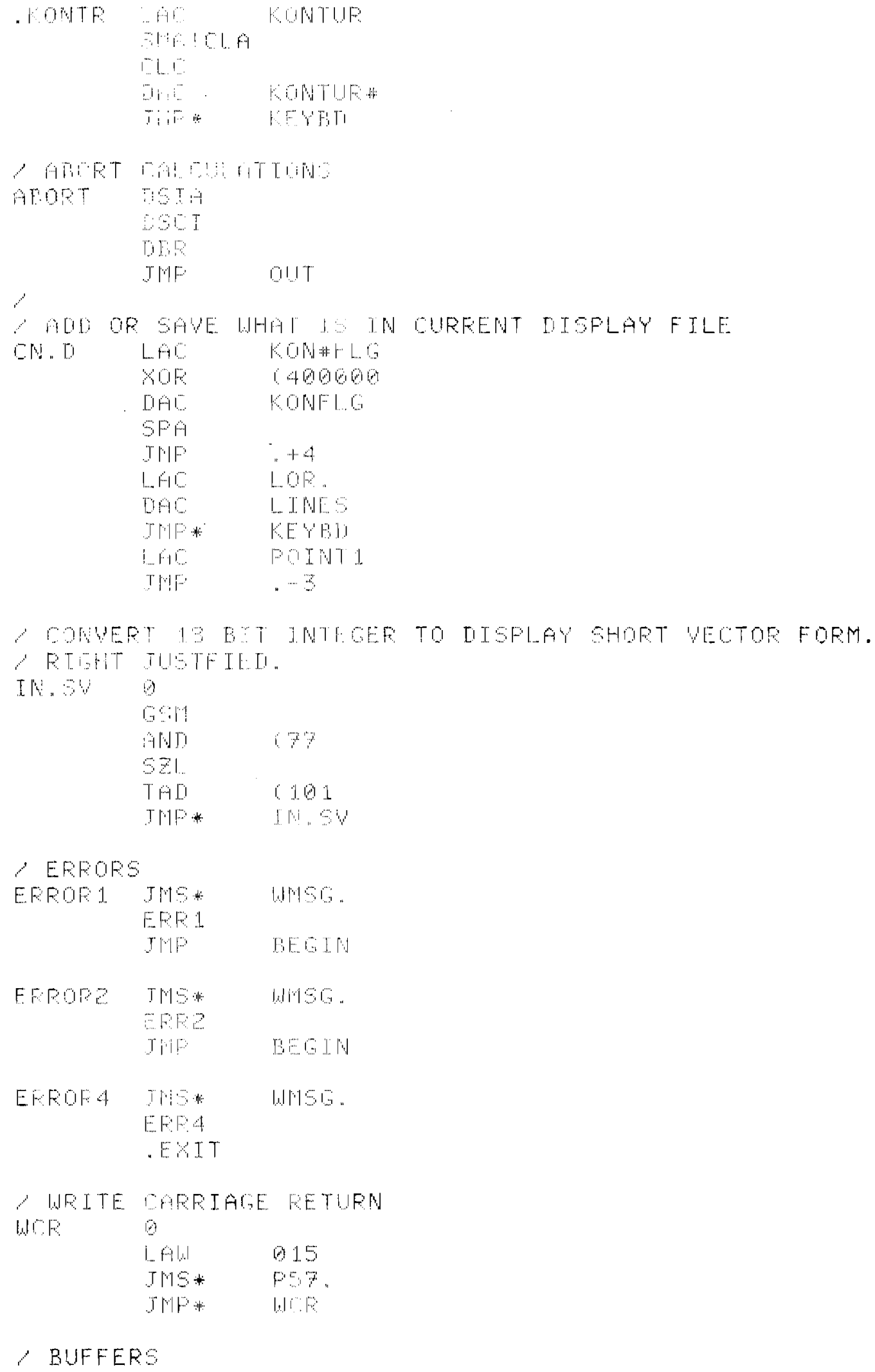




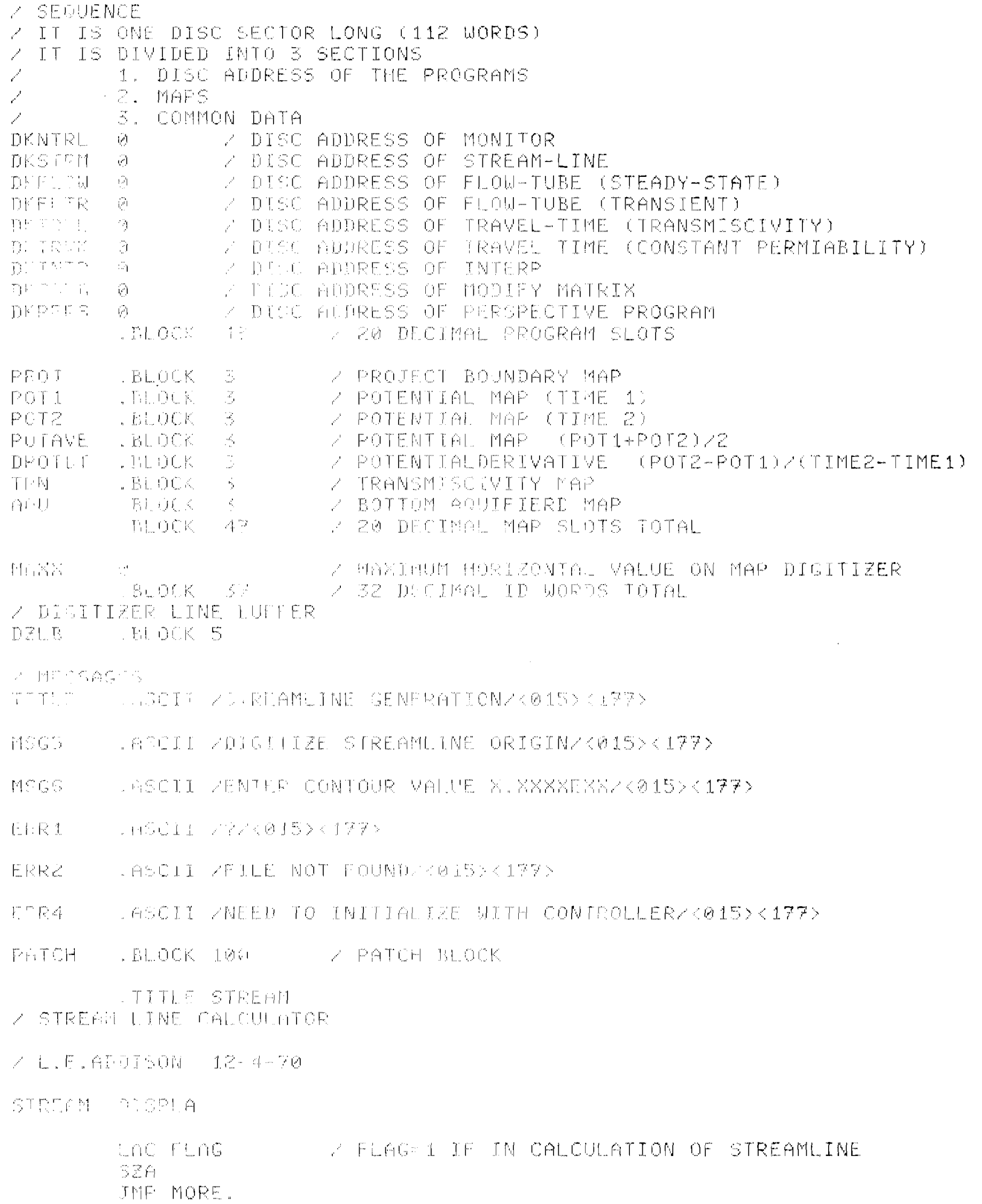




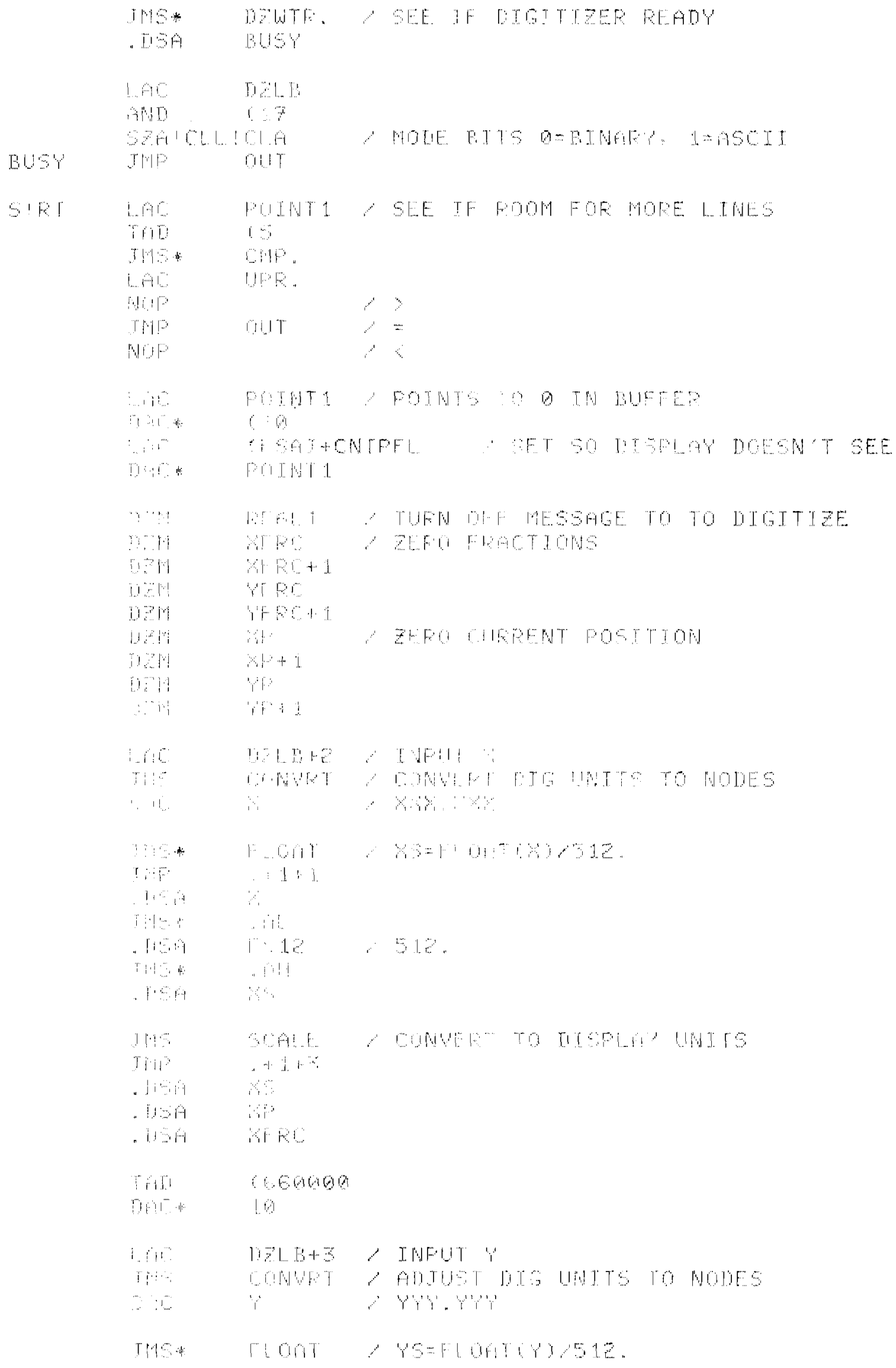




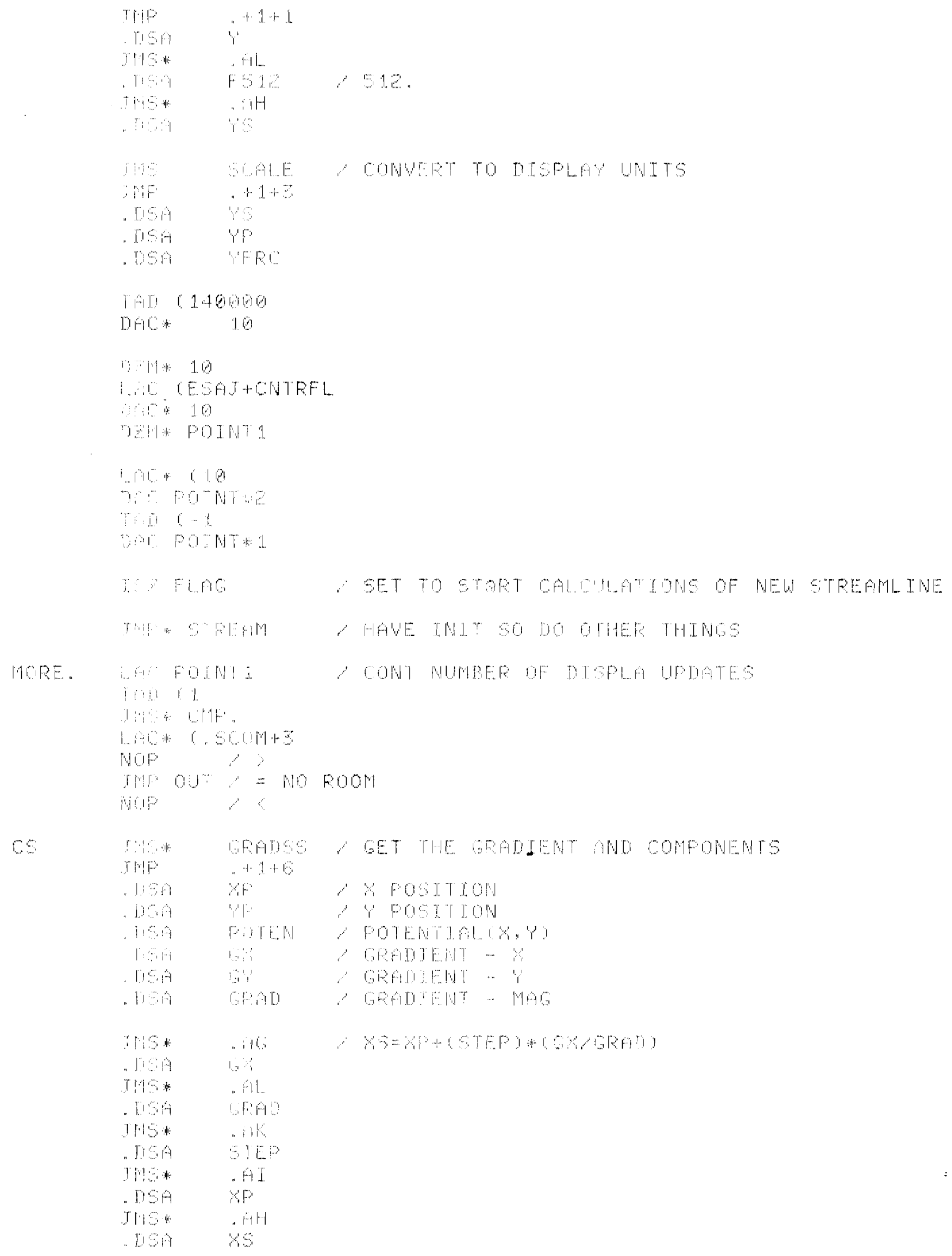




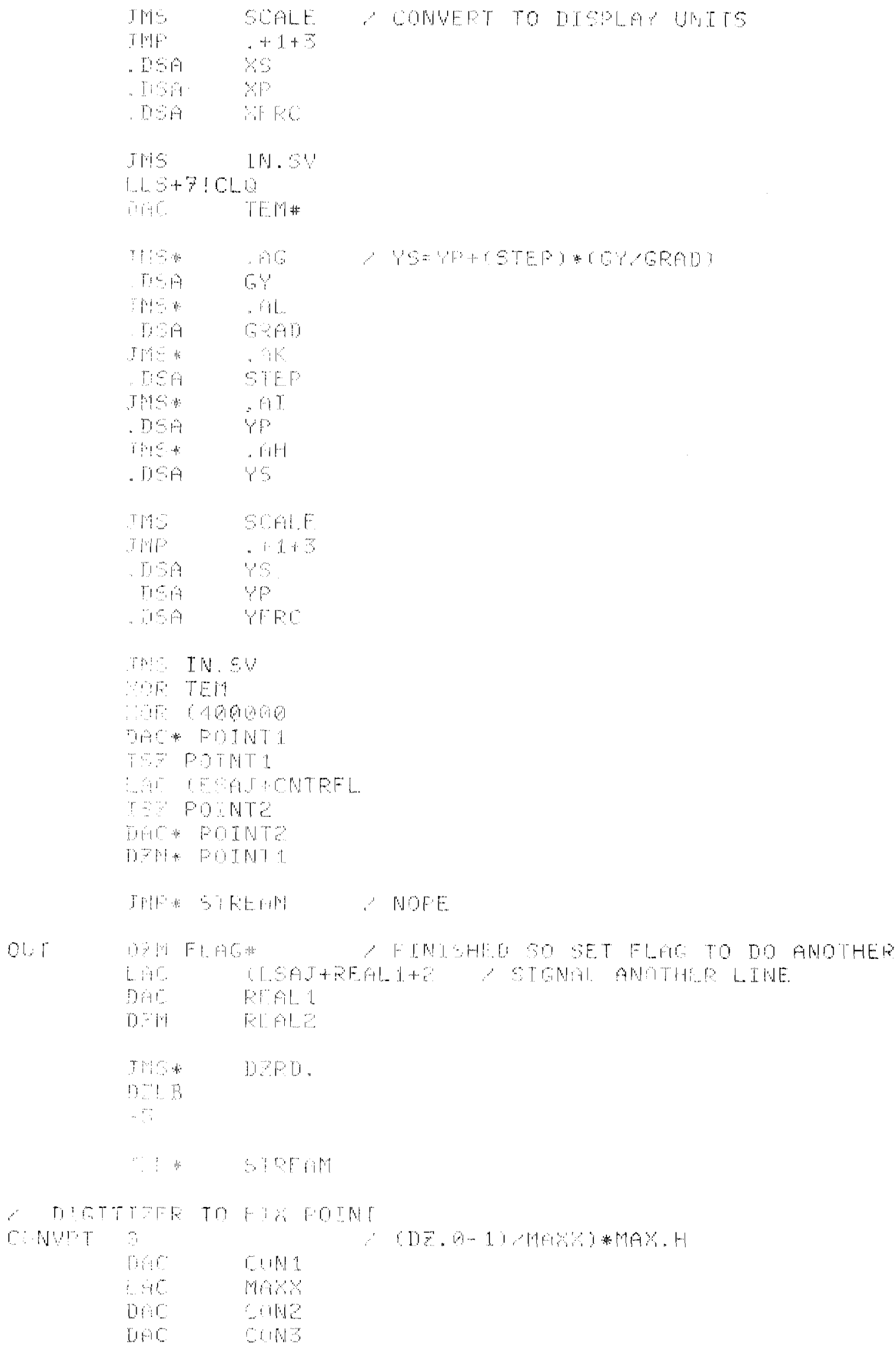




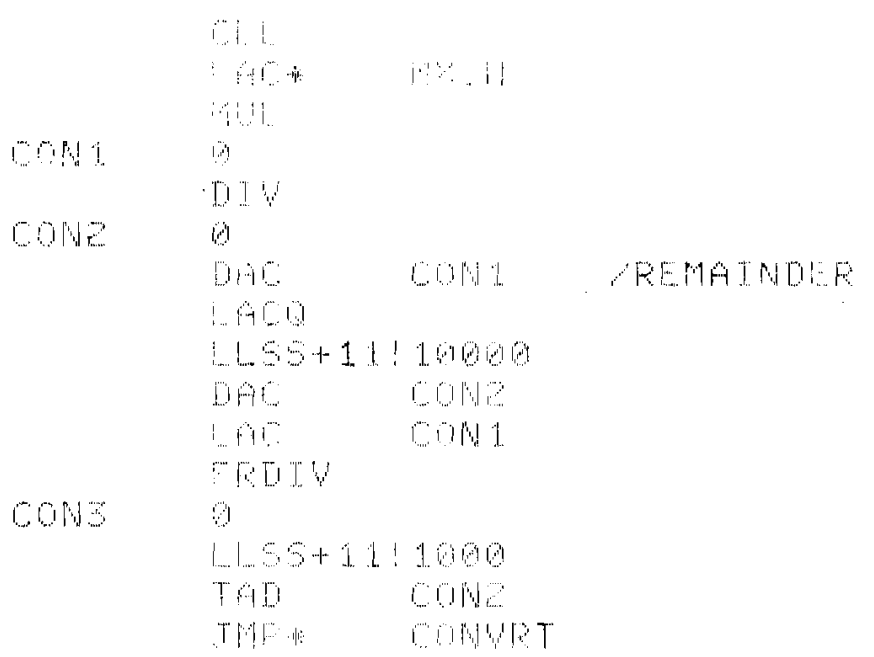




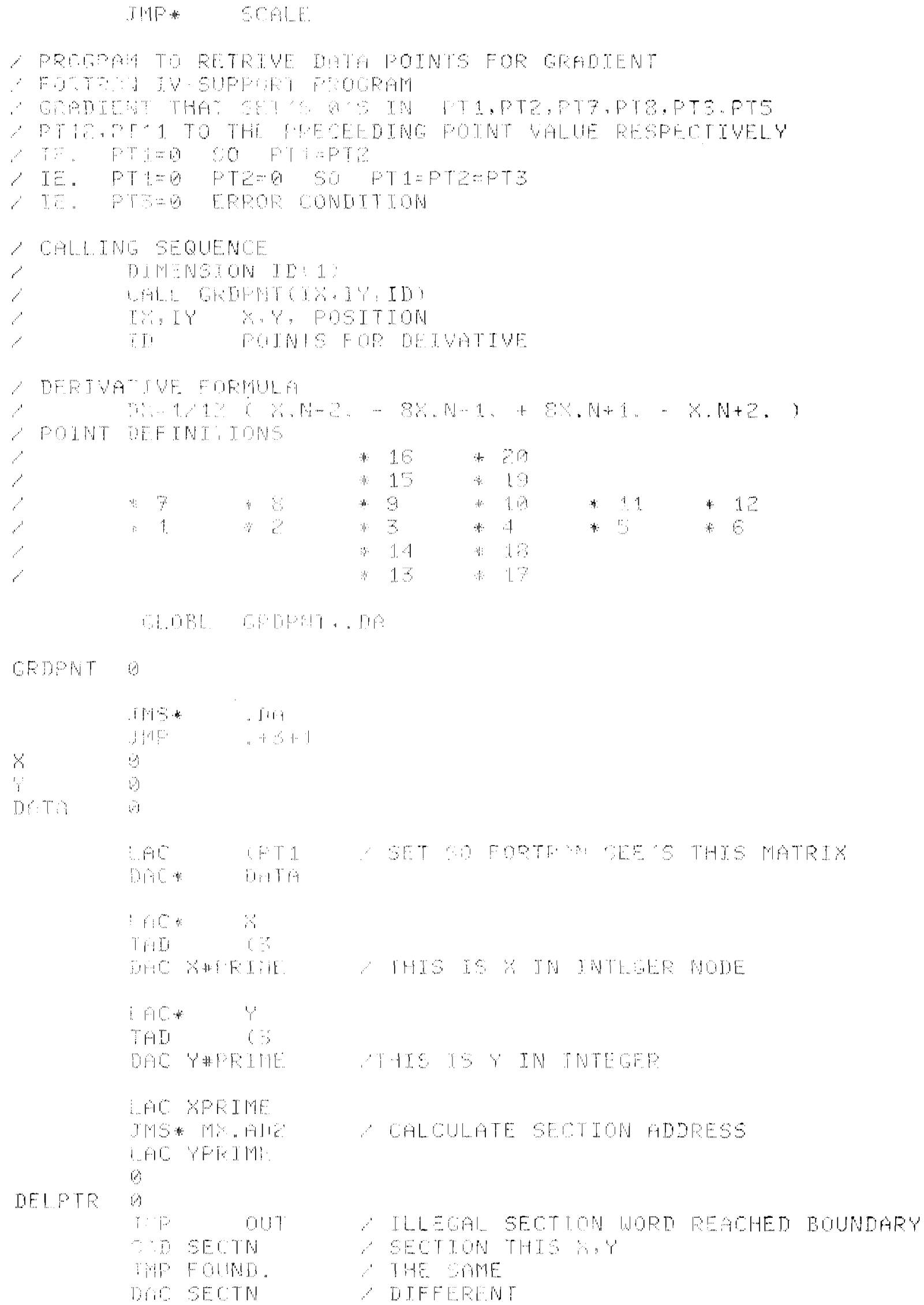




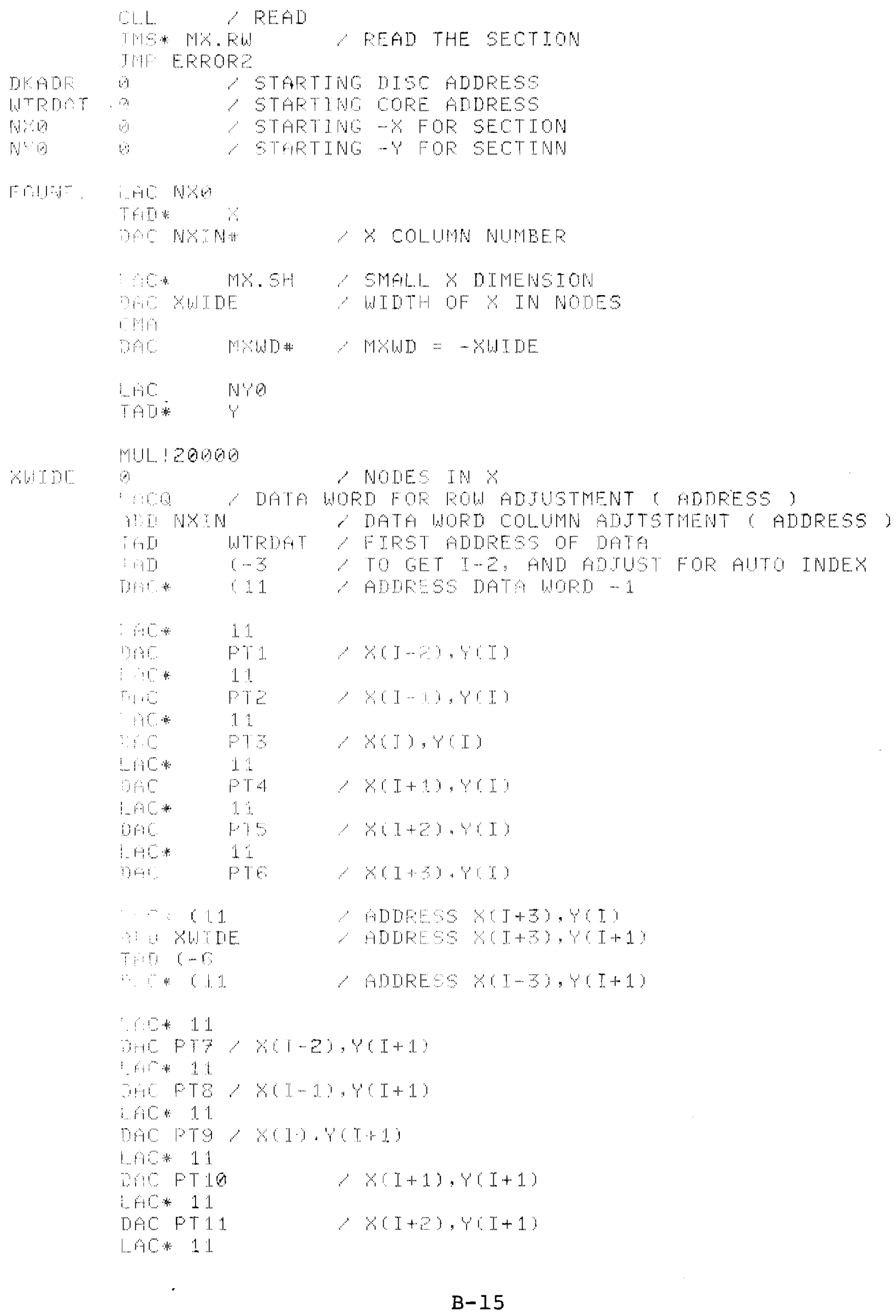




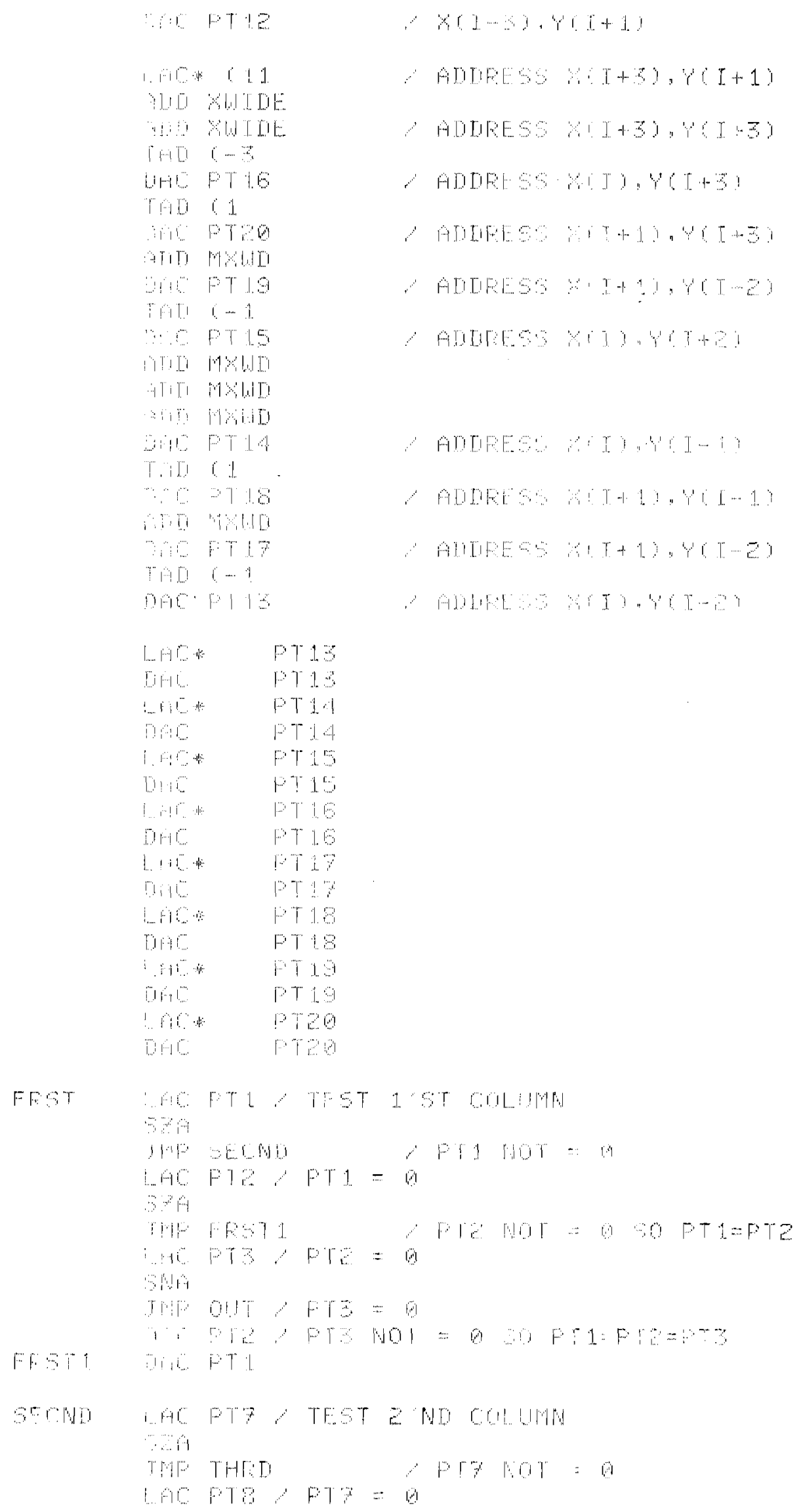




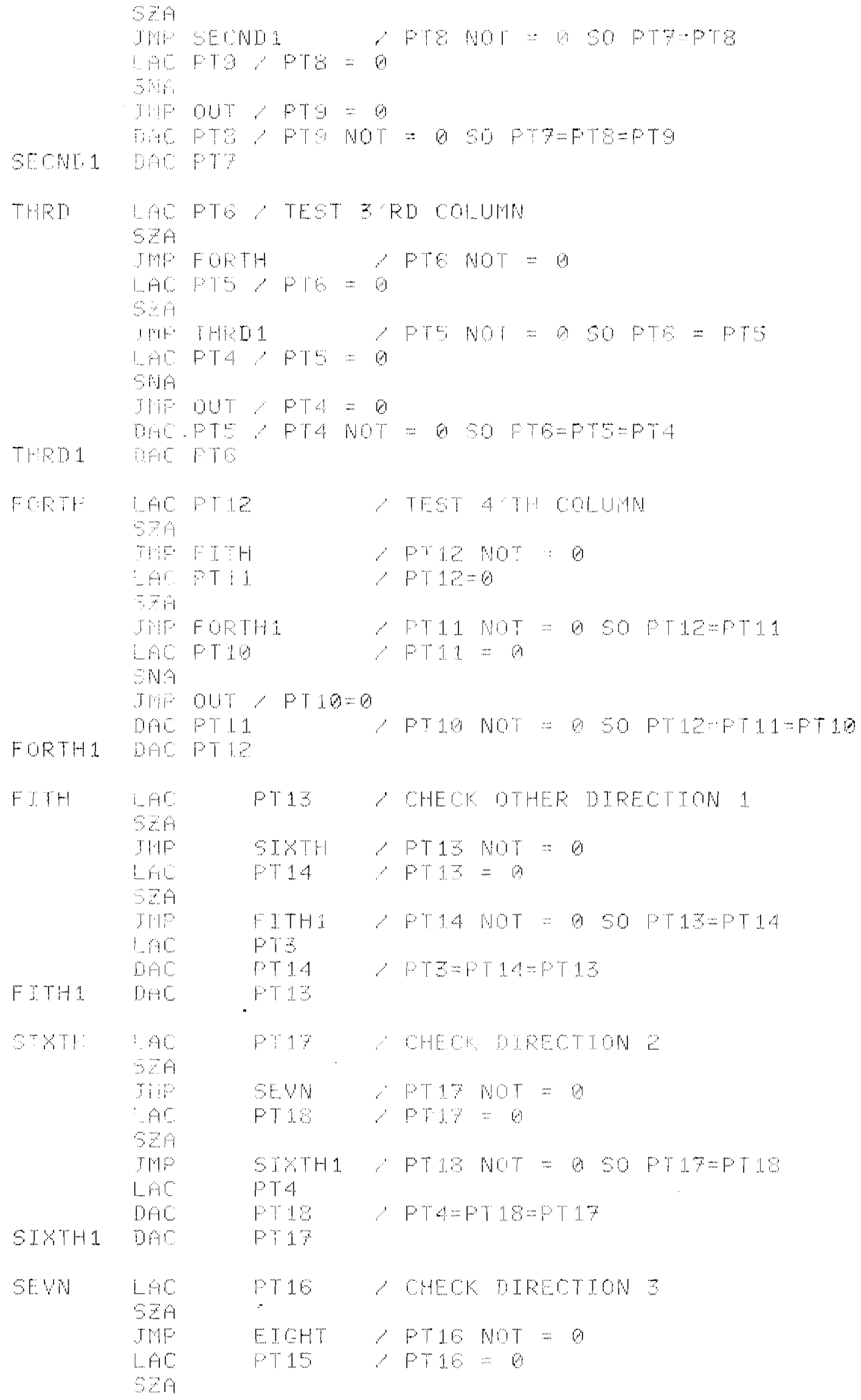




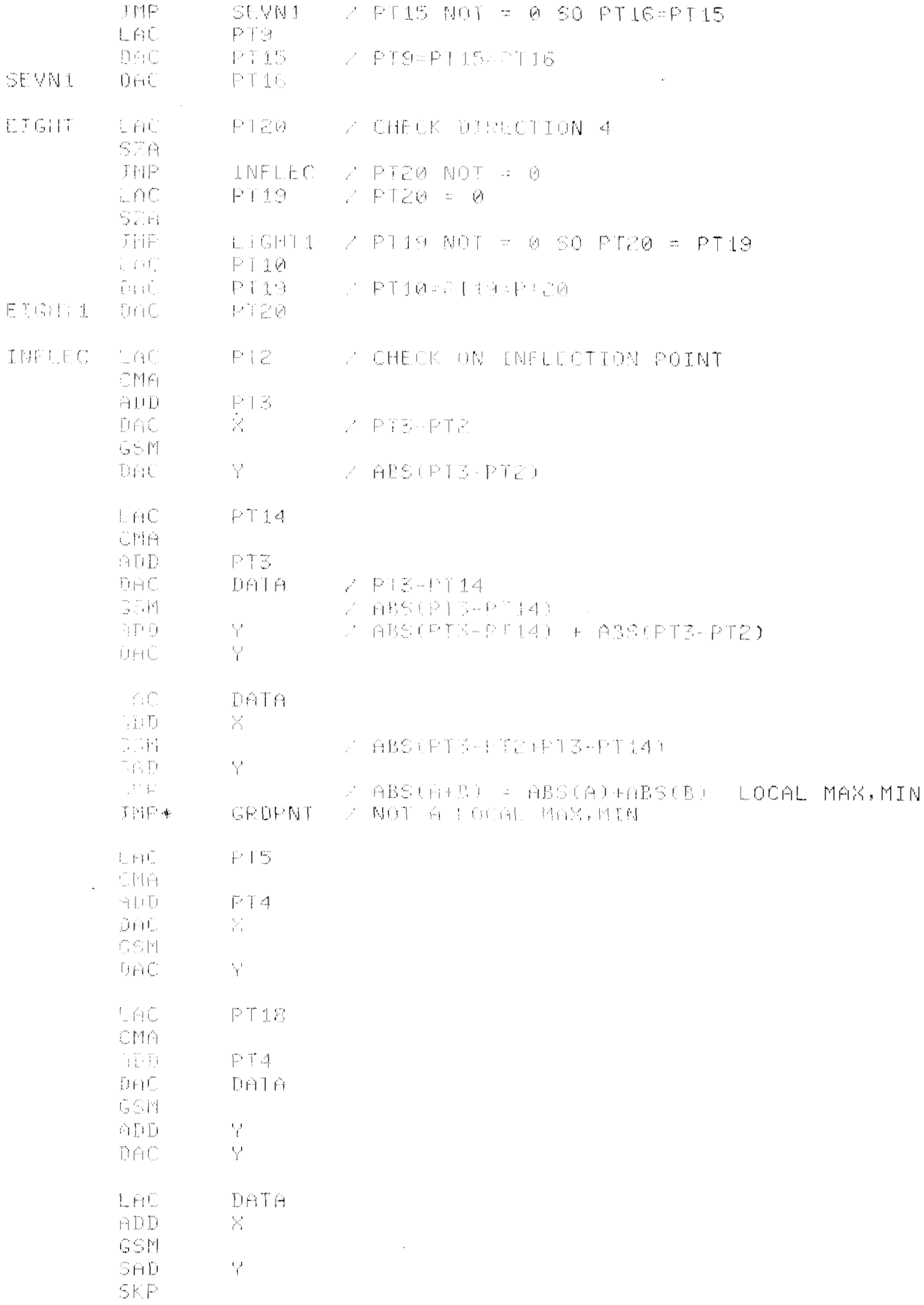




\begin{tabular}{|c|c|}
\hline THF & GFDPNT \\
\hline $\begin{array}{l}\mathrm{AC} \\
\mathrm{HA}\end{array}$ & FTB \\
\hline ADD & FT9 \\
\hline $\begin{array}{l}\text { DAC } \\
651\end{array}$ & $x$ \\
\hline $\mathrm{EAC}$ & $r$ \\
\hline $\begin{array}{l}\angle A C \\
C N A\end{array}$ & PT 15 \\
\hline$G D D$ & $\mathrm{PTS}$ \\
\hline $\begin{array}{l}D A C \\
95 \%\end{array}$ & DATA \\
\hline 田 & $Y$ \\
\hline 9110 & $r$ \\
\hline $\mathrm{AC}$ & DATA \\
\hline $\begin{array}{l}900 \\
69 \%\end{array}$ & \\
\hline $9 \mathrm{HD}$ & $Y$ \\
\hline 56 & \\
\hline TMP* & GRDPNT \\
\hline $\begin{array}{l}\text { LAO } \\
\text { CMA }\end{array}$ & $F T 11$ \\
\hline$A D D$ & $P T 10$ \\
\hline 960 & $x$ \\
\hline 500 & $Y$ \\
\hline $\begin{array}{l}A C \\
A B\end{array}$ & FT19 \\
\hline TIII & $F T 10$ \\
\hline 196 & DATA \\
\hline ATD & ' \\
\hline 506 & $Y$ \\
\hline LAC & DATA \\
\hline$A[I]$ & $x$ \\
\hline $65 \%$ & \\
\hline SAD & $Y^{\prime}$ \\
\hline एా & \\
\hline TMP & GRDPAT \\
\hline $0 \geq 1$ & $x$ \\
\hline $\begin{array}{l}\text { LAC } \\
\text { CHAG }\end{array}$ & FTE \\
\hline ADI & FTS \\
\hline $\begin{array}{l}694 \\
82 \mathrm{~L}\end{array}$ & \\
\hline$I S Z$ & $x$ \\
\hline $\begin{array}{l}\mathrm{LAC} \\
\mathrm{CM}\end{array}$ & PTS \\
\hline
\end{tabular}

B- 19 


\begin{tabular}{|c|c|}
\hline 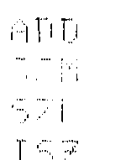 & $P^{\top} 4$ \\
\hline & \\
\hline 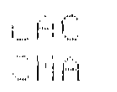 & $P T B$ \\
\hline 6 & $\% 79$ \\
\hline $\begin{array}{l}5 \% 11 \\
321\end{array}$ & \\
\hline 19 & $x$ \\
\hline $\begin{array}{l}A C \\
-M H\end{array}$ & $P T 11$ \\
\hline $\begin{array}{l}915 \\
6 \% 1 \\
51\end{array}$ & $P T 10$ \\
\hline$T S Z$ & $x$ \\
\hline $\begin{array}{l}\text { मी० } \\
\mathrm{HW}\end{array}$ & $\%$ \\
\hline T & OU1 \\
\hline $\begin{array}{l}9 F \mathrm{WH} \\
9 \mathrm{~F}\end{array}$ & $i 4$ \\
\hline T THF & GFDOTT \\
\hline 01 & $x$ \\
\hline 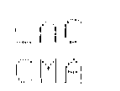 & 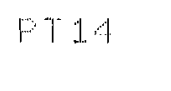 \\
\hline 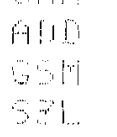 & {$[\cdots$} \\
\hline$T 67$ & $\%$ \\
\hline $\begin{array}{l}\ldots \\
\cdots \\
\cdots \\
\cdots\end{array}$ & $\% T j$ \\
\hline $\begin{array}{c}110 \\
\cdots \\
\cdots \\
\cdots\end{array}$ & P" \\
\hline$P: Y^{\prime}$ & 4 \\
\hline $\begin{array}{r}\therefore i \ldots \\
\because f\end{array}$ & $P+1 \vdots$ \\
\hline $\begin{array}{l}901 \\
691 \\
321\end{array}$ & $\beta T 9$ \\
\hline $15 z$ & 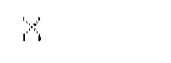 \\
\hline $\begin{array}{l}\qquad A C \\
m !\end{array}$ & $P+10$ \\
\hline 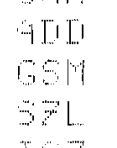 & $\mathrm{Frg}$ \\
\hline $1-z$ & $\mathrm{r}$ \\
\hline 1... & $x_{1}$ \\
\hline
\end{tabular}




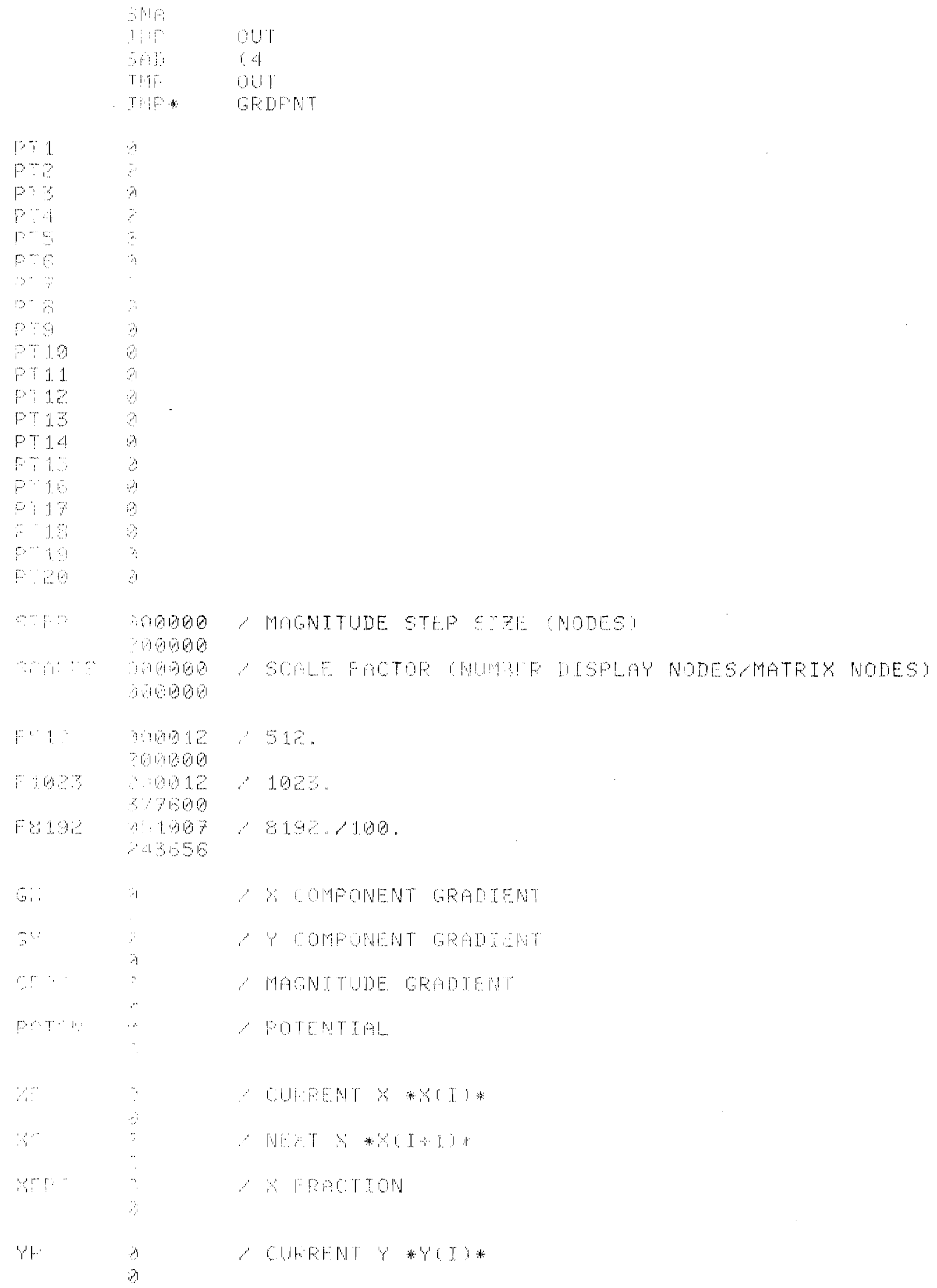

B-2I 


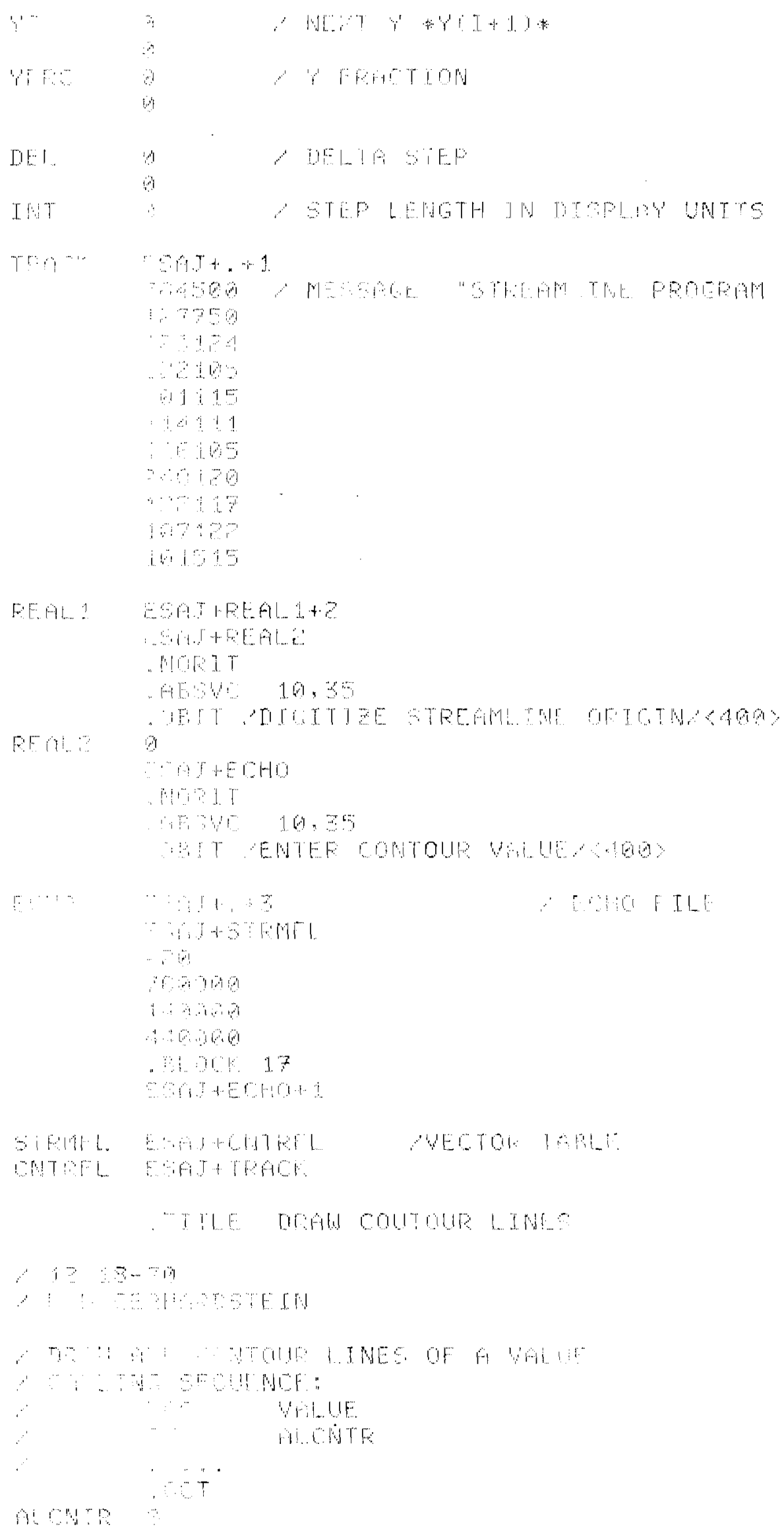




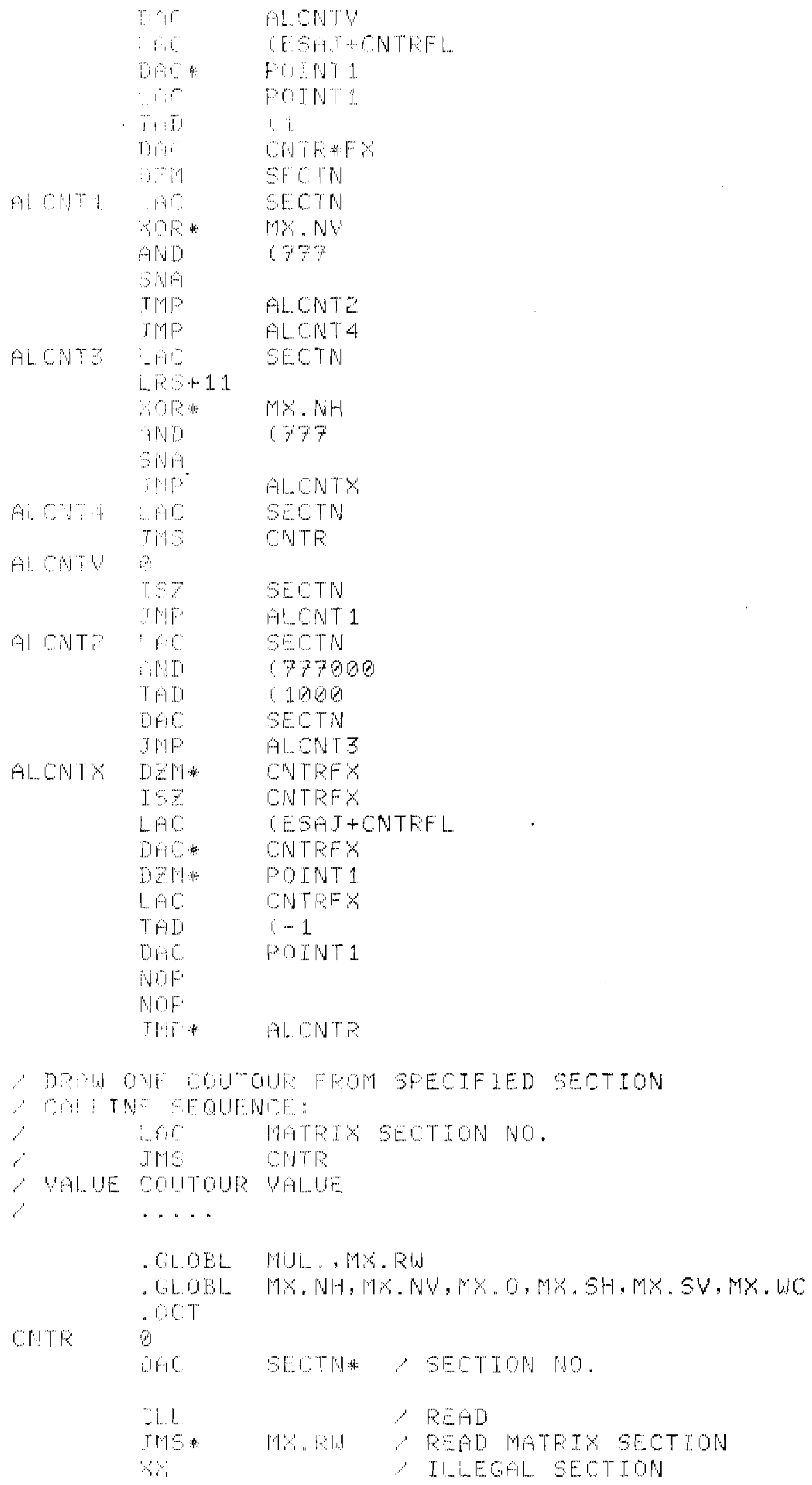




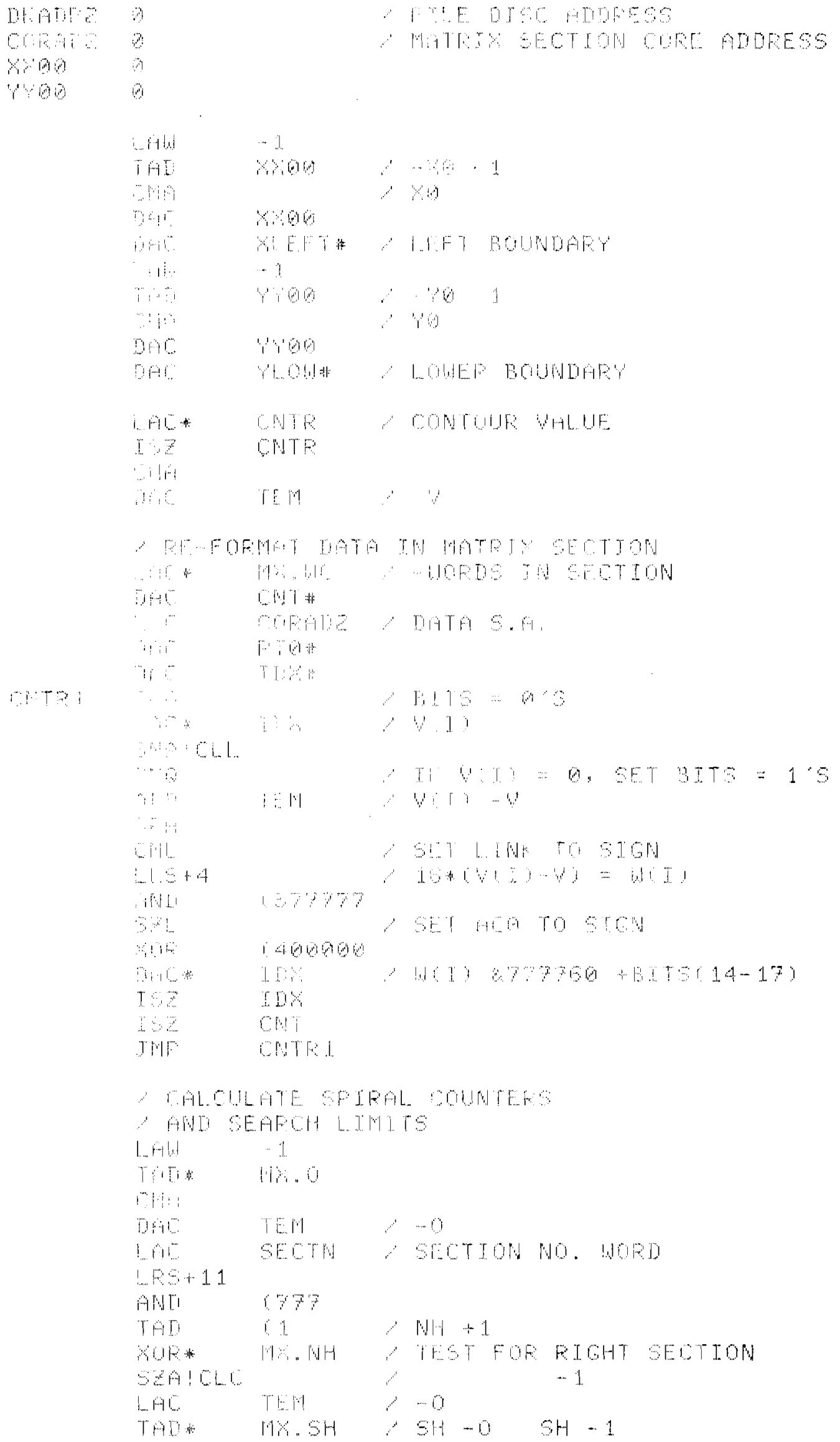




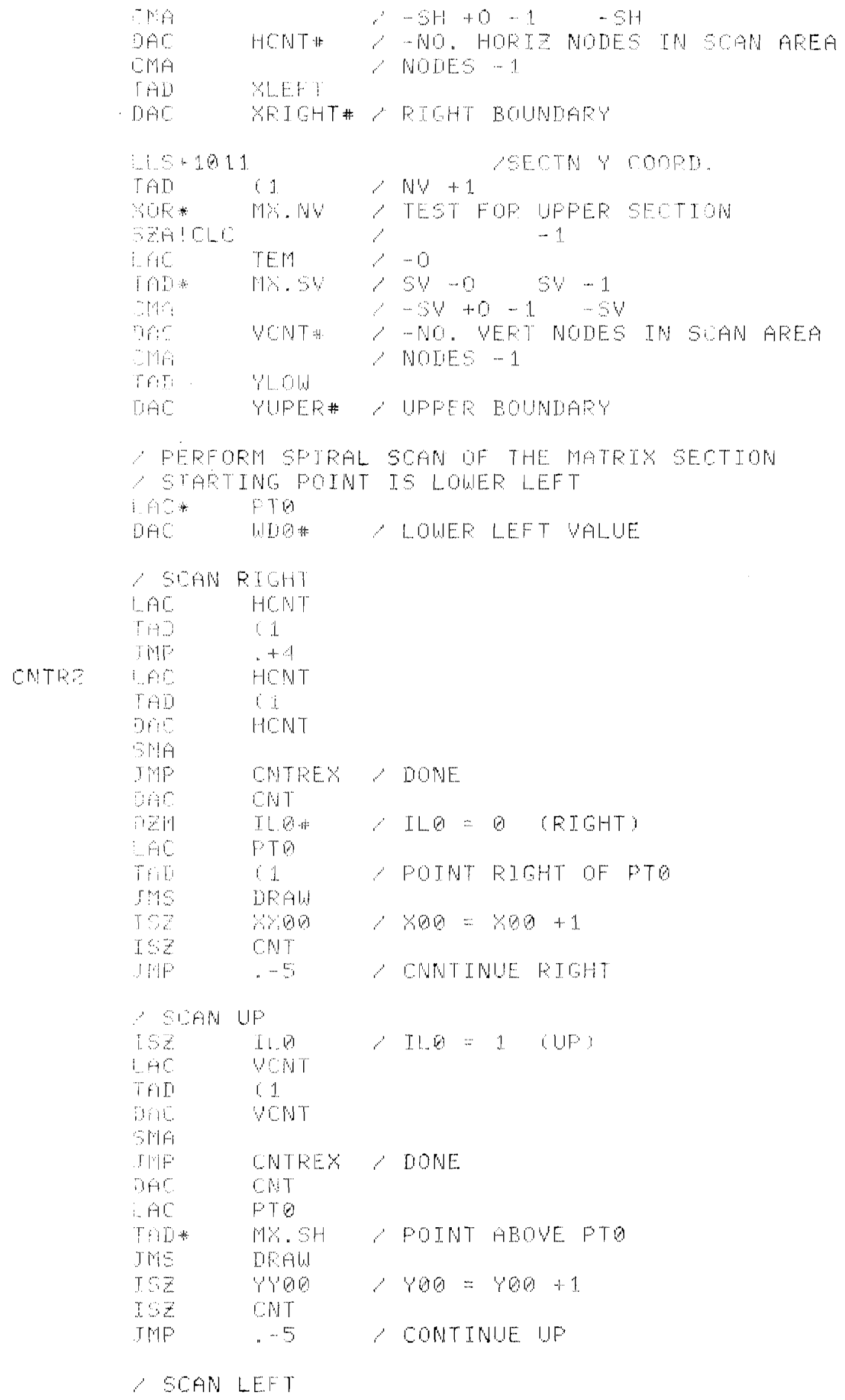




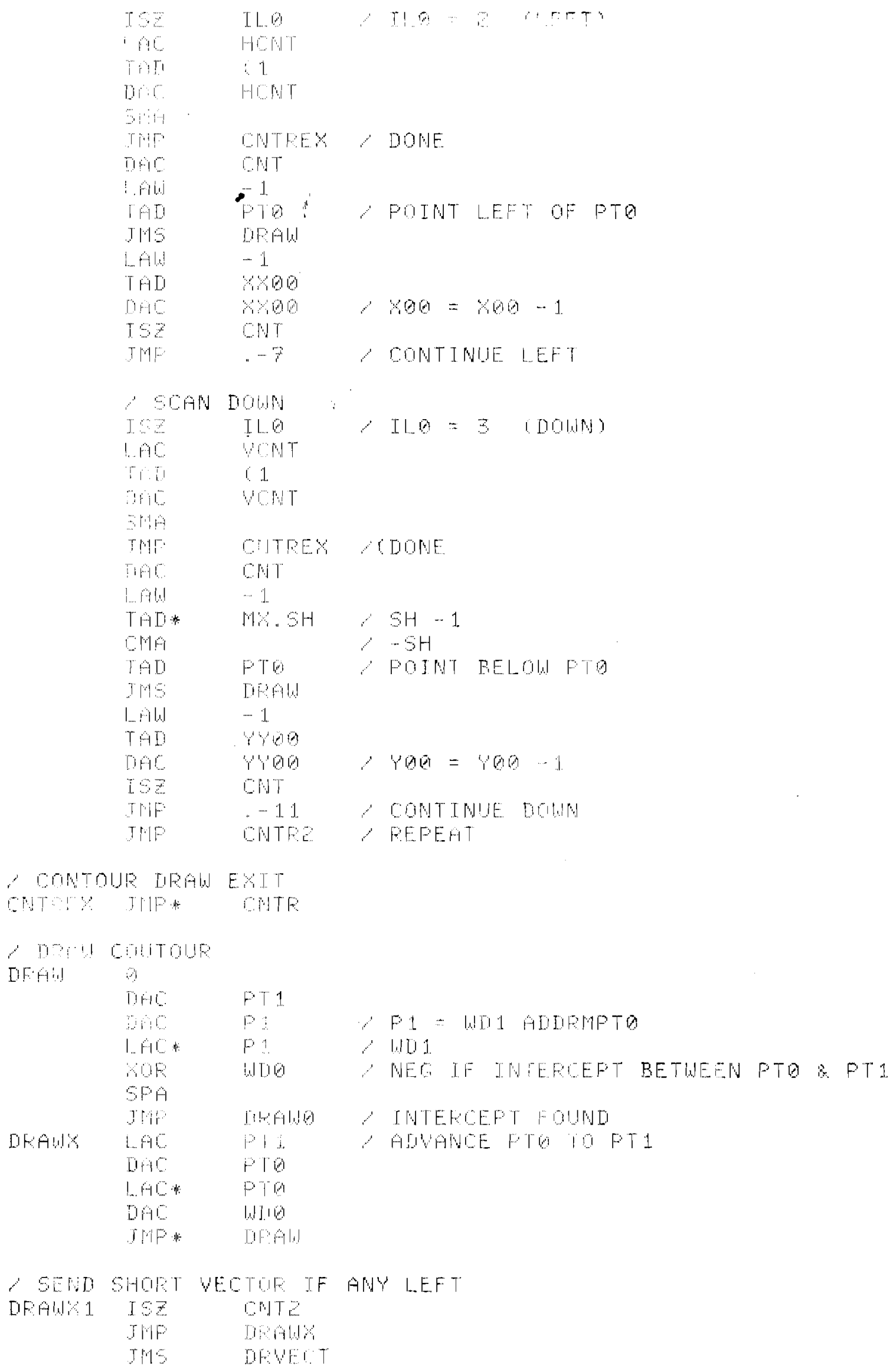




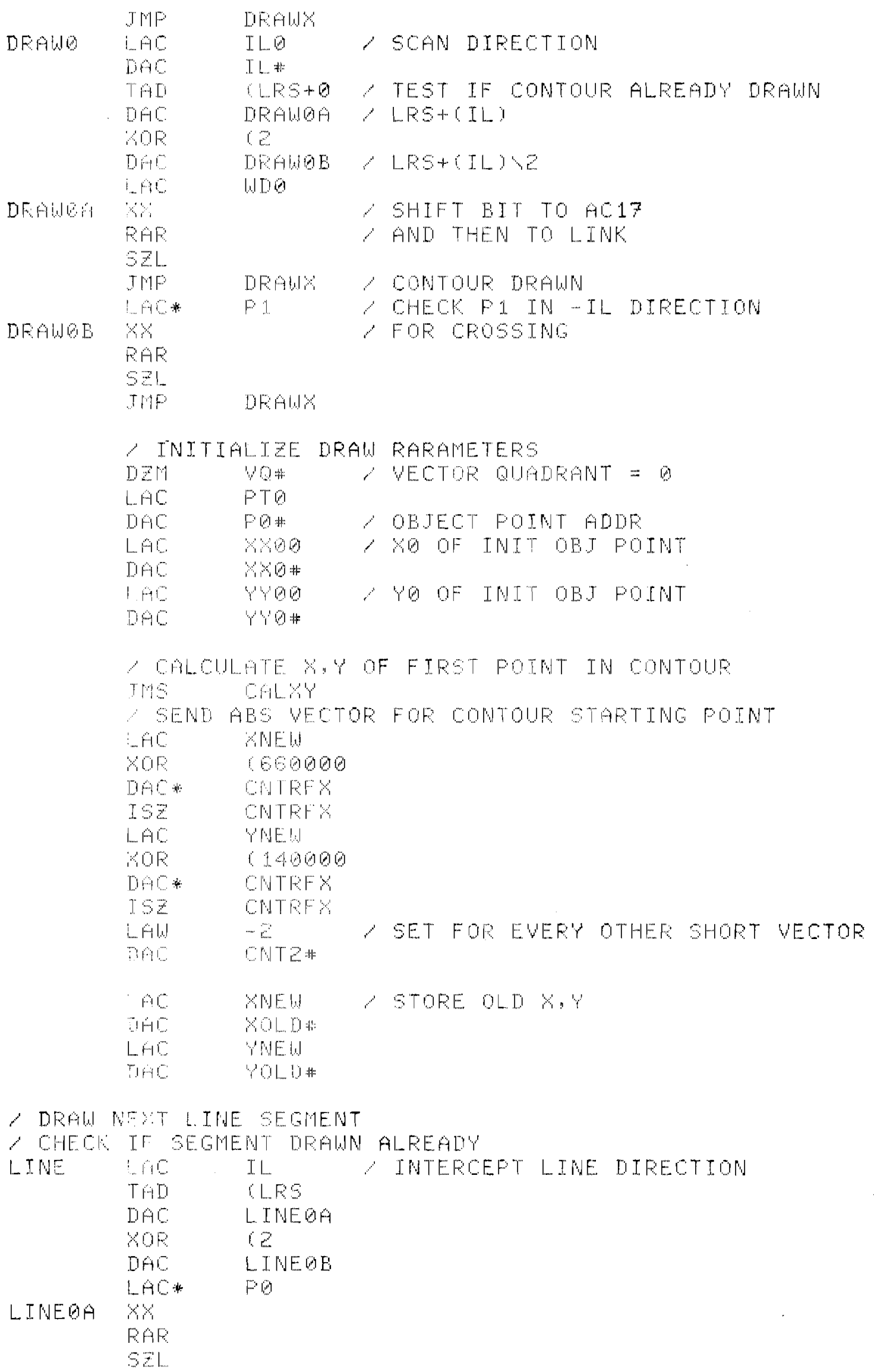




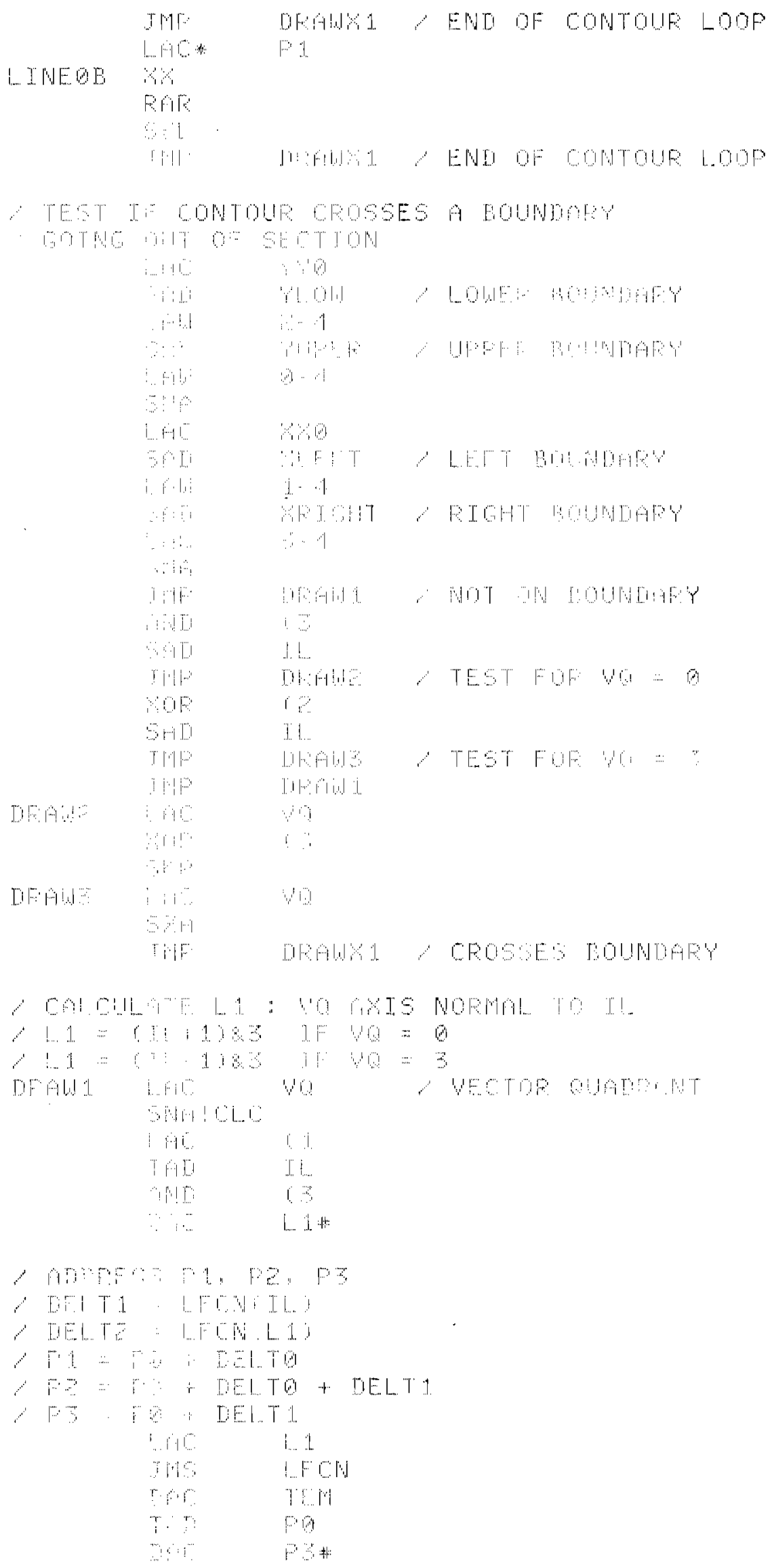




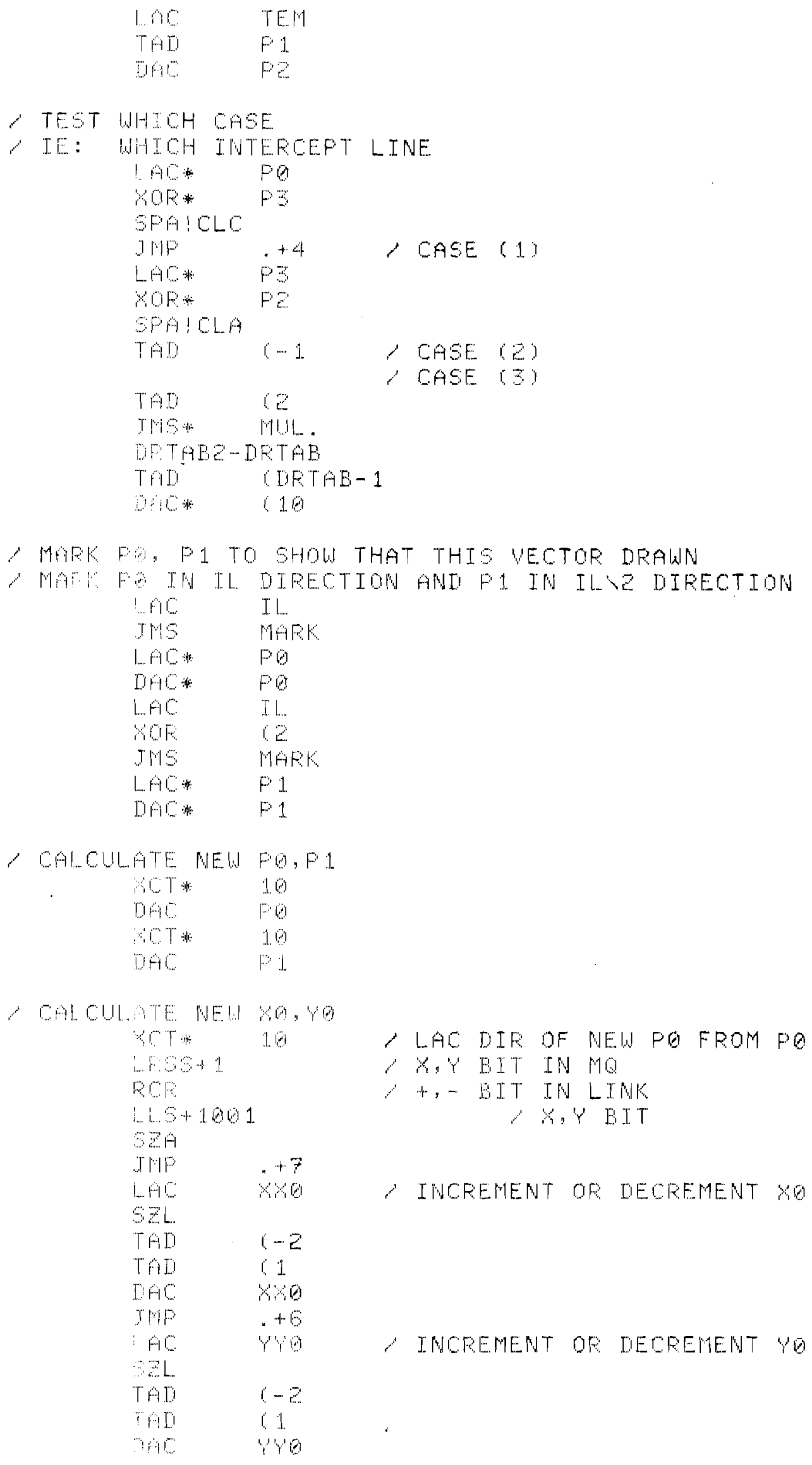




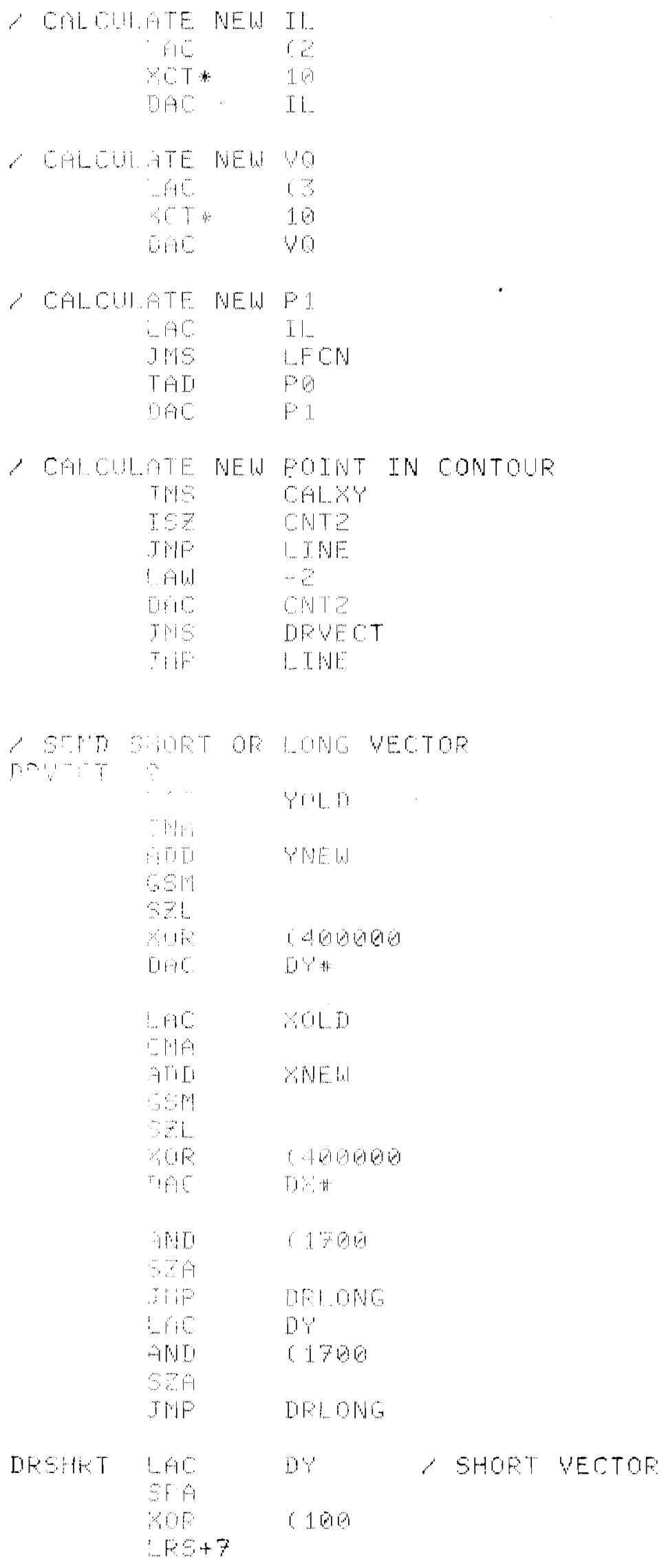




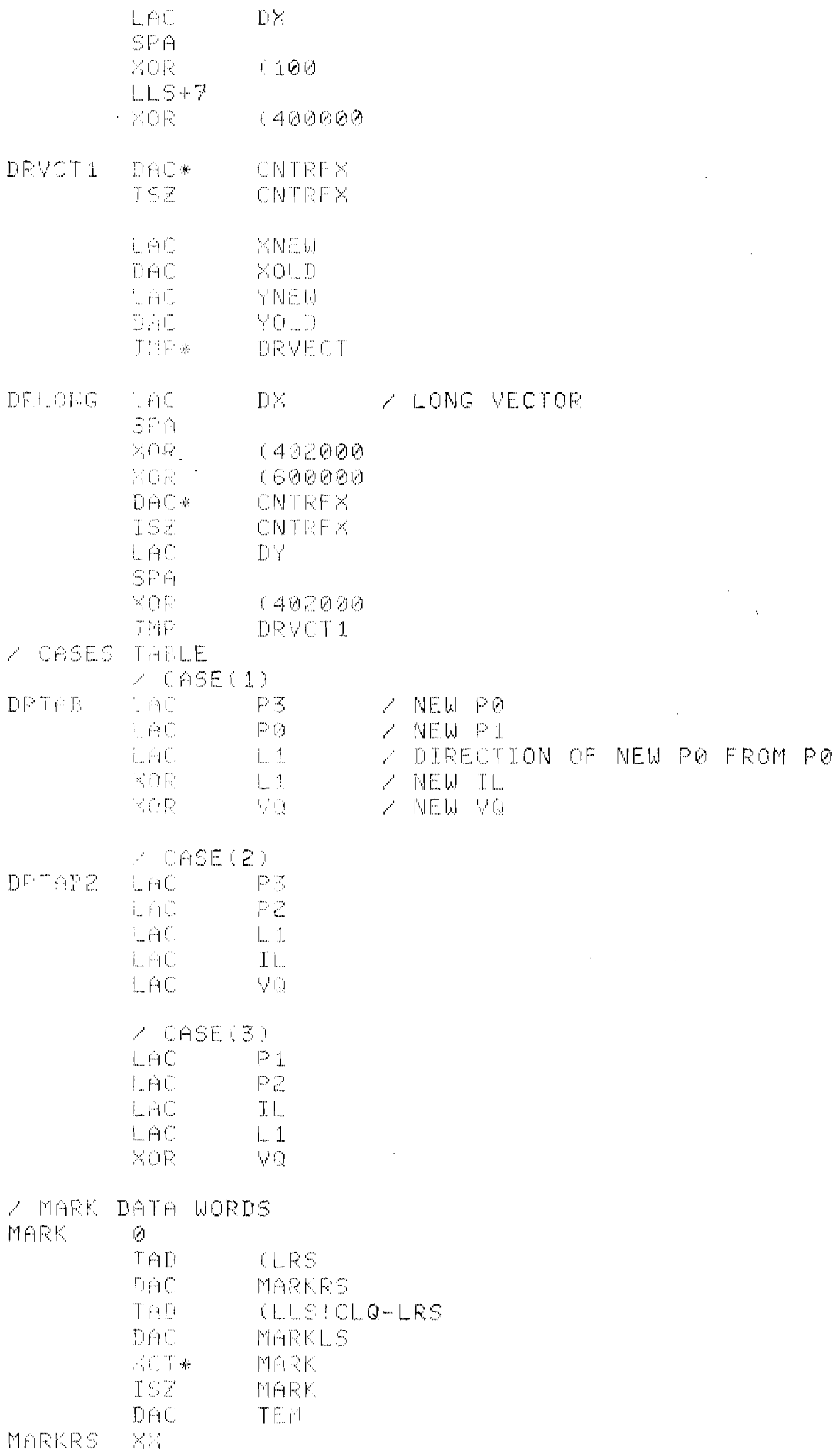




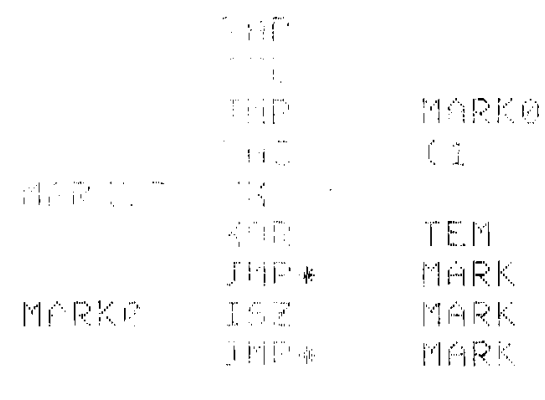

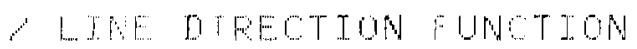

L..........

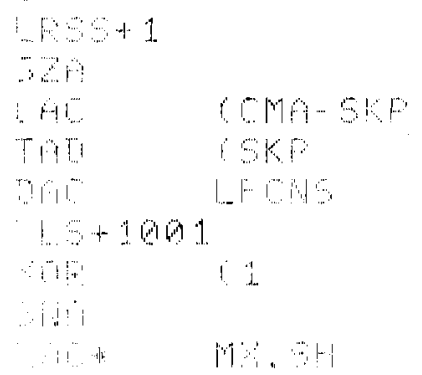

i. 1.1

11

Thi

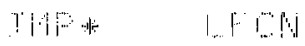

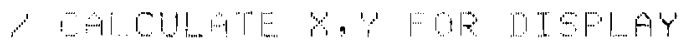

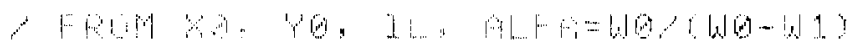

C. Hin

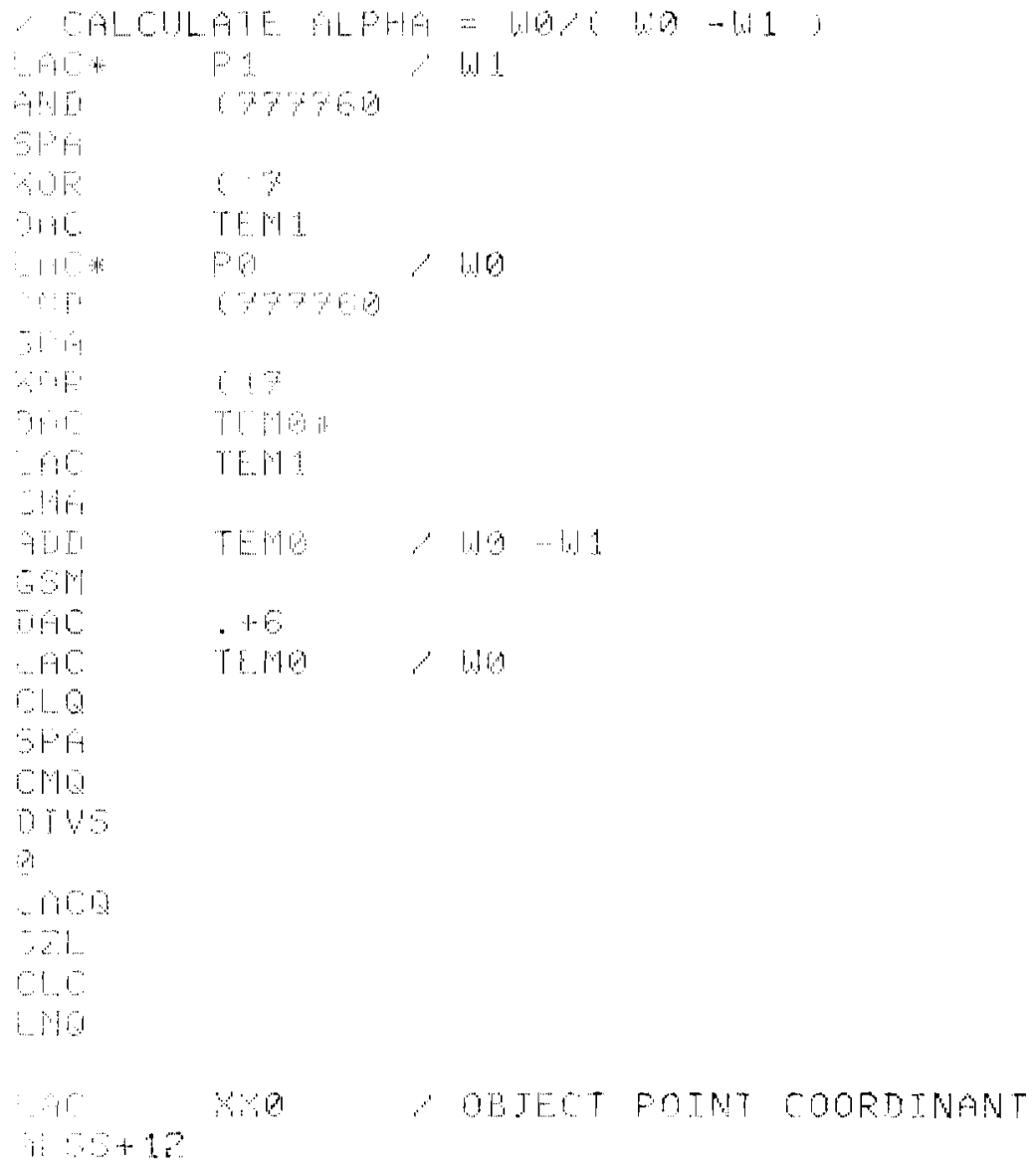




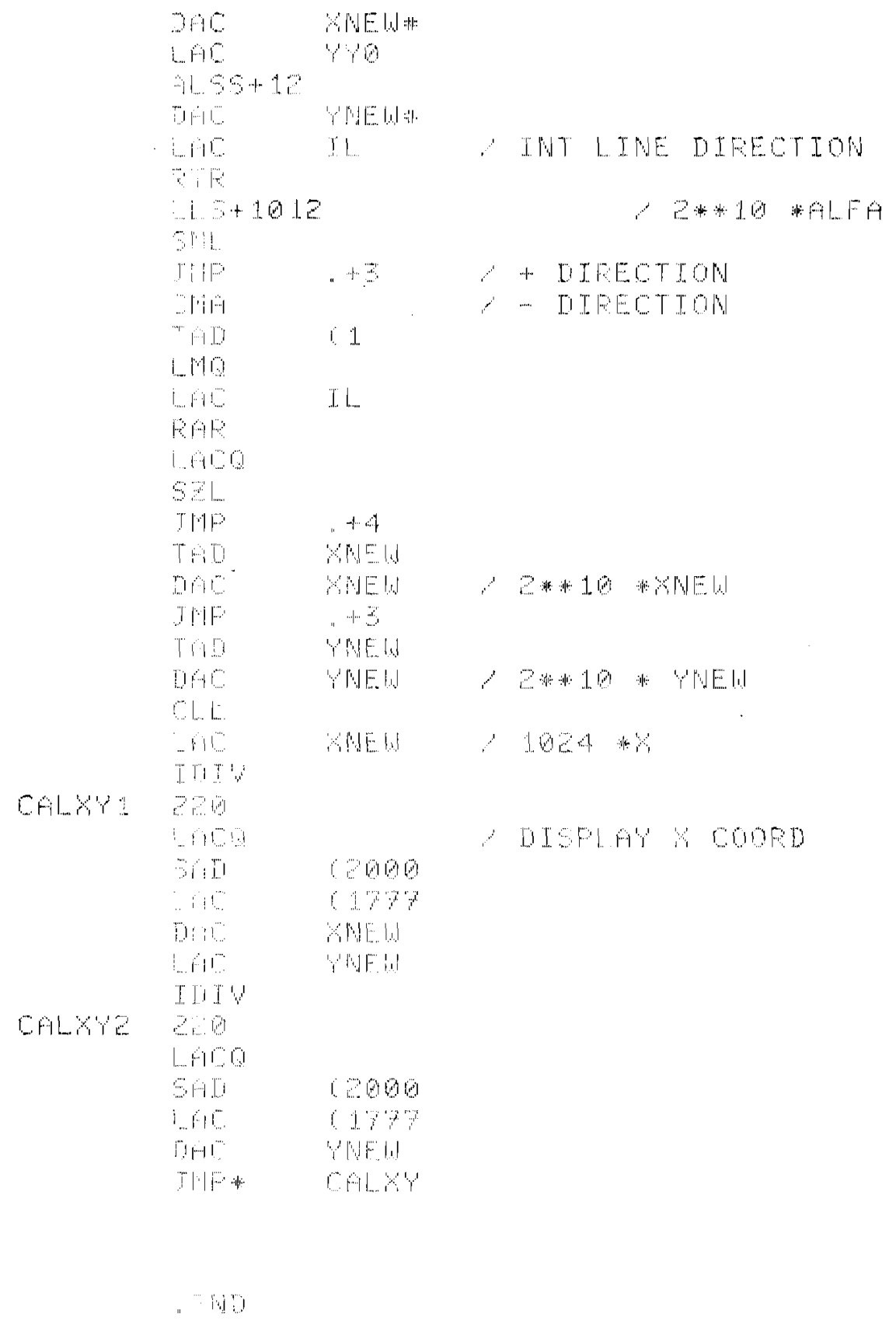

$B-33$ 
APPENDIX C

TRAVEL TIME PROGRAM LISTING 


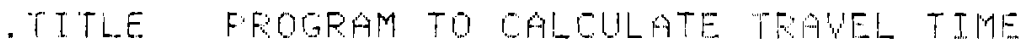

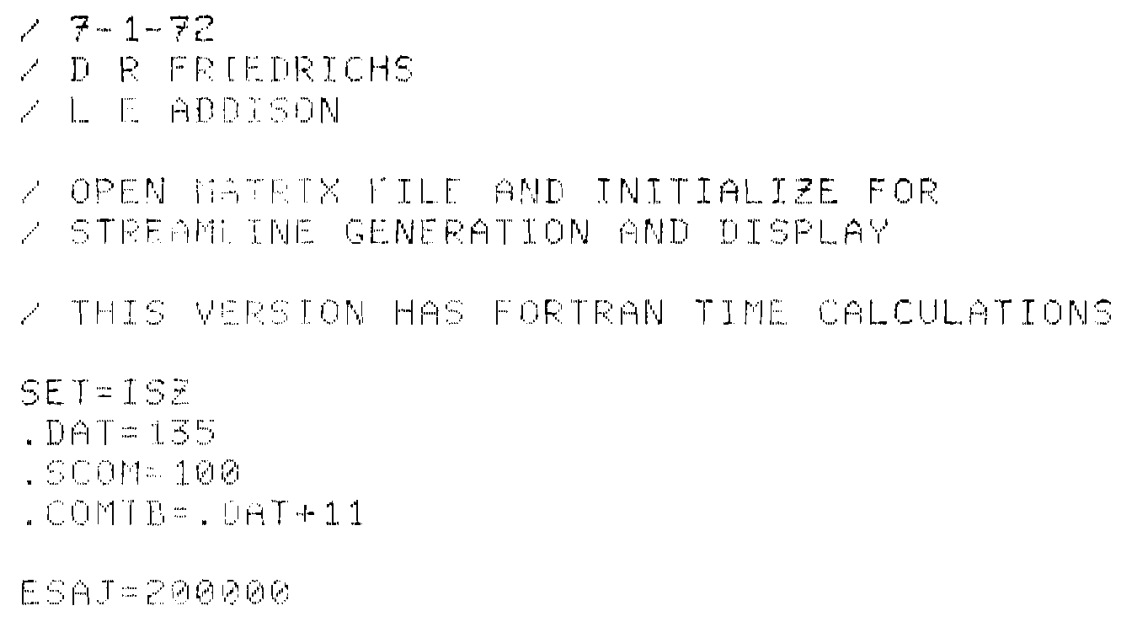




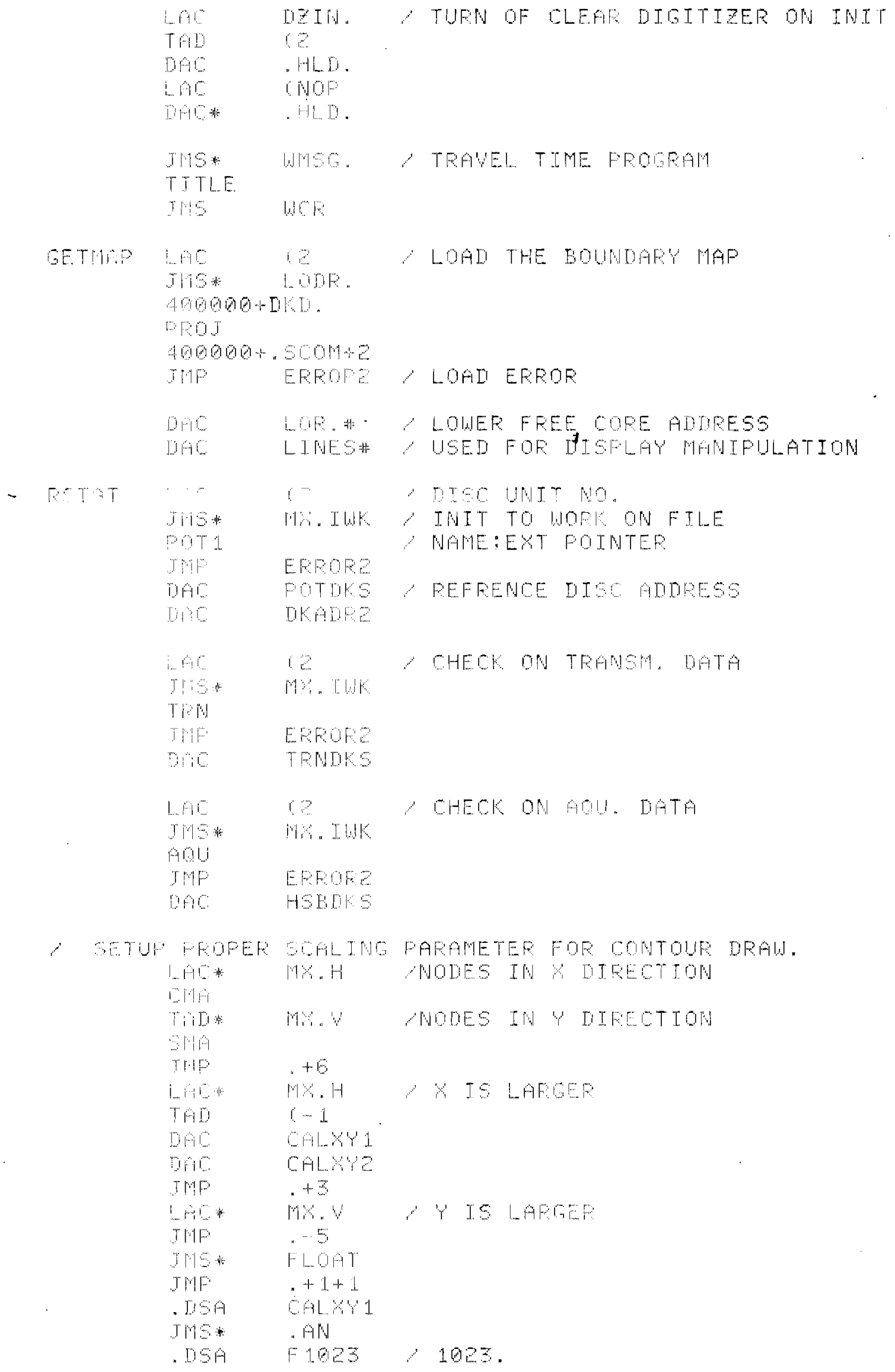




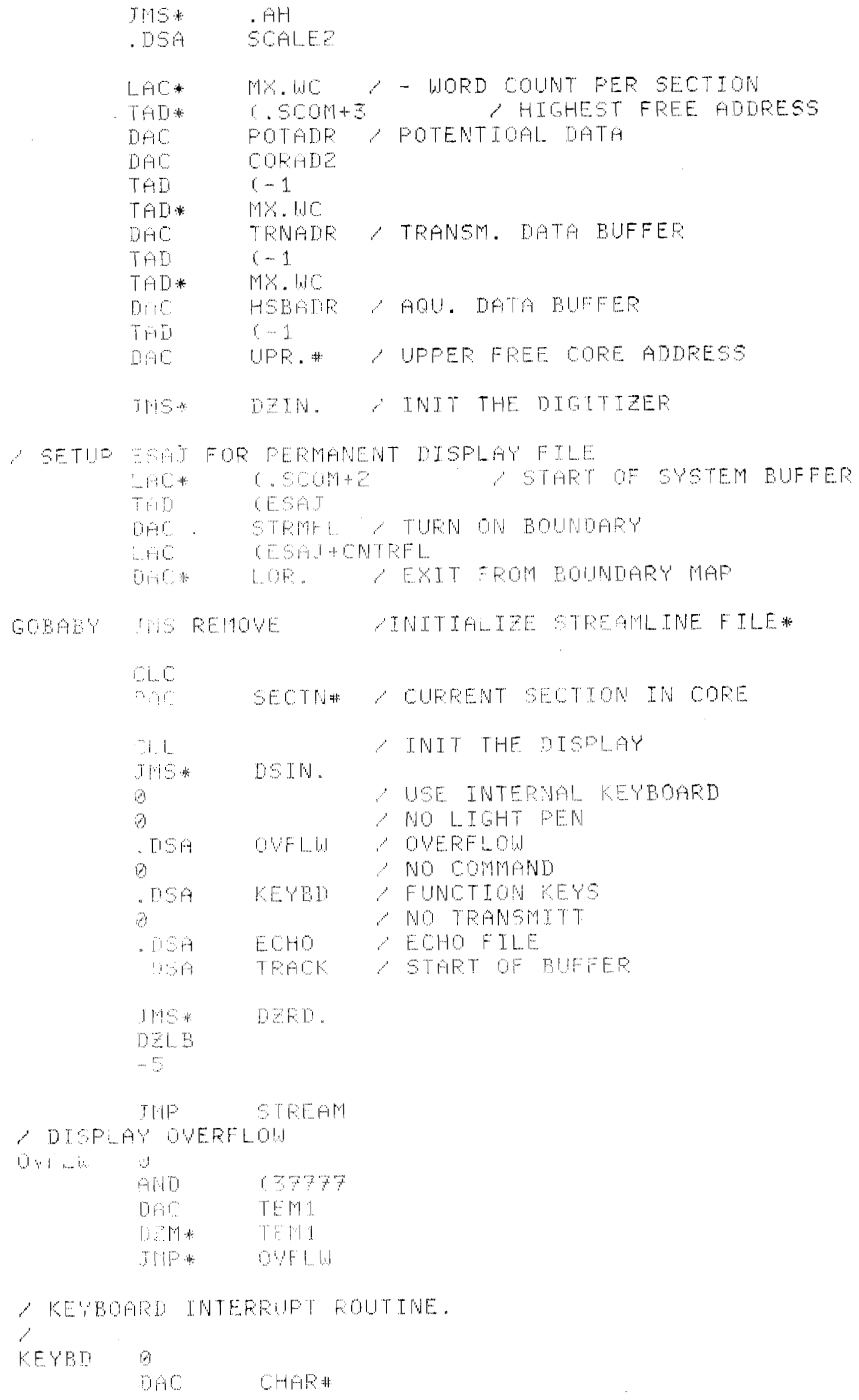




\begin{tabular}{|c|c|c|}
\hline & $3 \| 1$ & $K 1 B L+1$ \\
\hline & $\mathrm{J} 川$ & धन Тि \\
\hline & 1.69 & बिF \\
\hline & $9 \beta$ & \\
\hline & Ill & \% W \\
\hline & $1.91 \%$ & ब्ति \\
\hline & 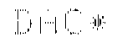 & 115 \\
\hline & 1110 & К⿴囗十 \\
\hline & "yनl一: & 116 \\
\hline KYBTP & LAC & 15 \\
\hline & की & \\
\hline & $\pi \| \%$ & 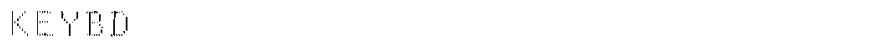 \\
\hline & $3 \mathrm{AD}$ & E+ta \\
\hline & $\mathrm{J}^{\prime}\left|\mathrm{y}^{\prime}\right|$ &. \\
\hline & {$\left[\begin{array}{l}a \\
0\end{array}\right.$} & 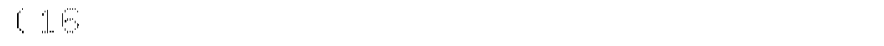 \\
\hline & T刉F' & KYBDLF \\
\hline & I & 16 \\
\hline КТТР & GH & \\
\hline & पिए & धTF \\
\hline & Tí & Eए巴ए \\
\hline$K F G$ & 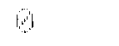 & \\
\hline $1 \mathrm{E}$ & 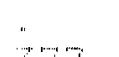 & \\
\hline & 17 & 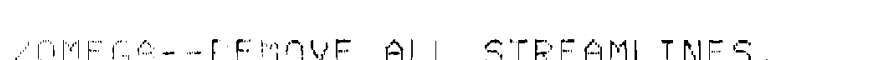 \\
\hline & 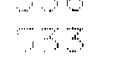 & 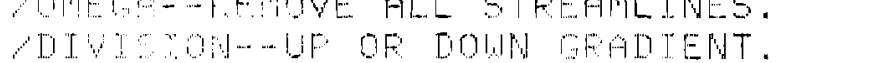 \\
\hline & 96 & 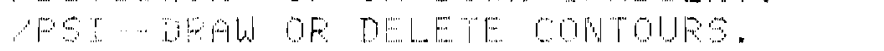 \\
\hline & $5 x$ & LFWDW- ENTEF STEF WOTE \\
\hline & $3+6$ & 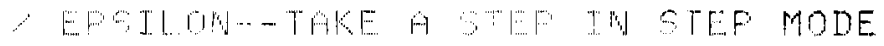 \\
\hline & 50 & $\therefore F+-1 M T$ \\
\hline & बi & 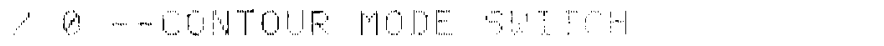 \\
\hline & 4 & 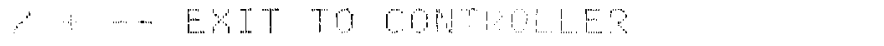 \\
\hline & 91 & 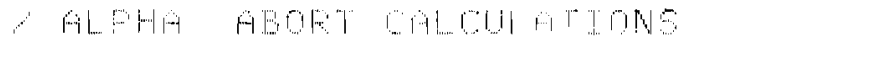 \\
\hline
\end{tabular}

$r$

KTTL

THF

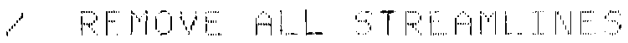
THF HO

Y CHAOE GPADE "WF GAOH

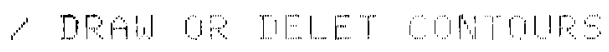
$M \%$

YENEP-LEAE STEF POTE JTHF $\quad .97 \mathrm{TH}$

THE A TEP

$\because$ LITT

JiF $\quad$, T F F

IWE SII TH

$\therefore$ CONTOUP WOE JW

$\Rightarrow$ EXTT TO OPTFOLLE TlF

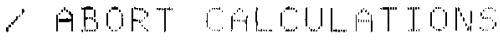
I

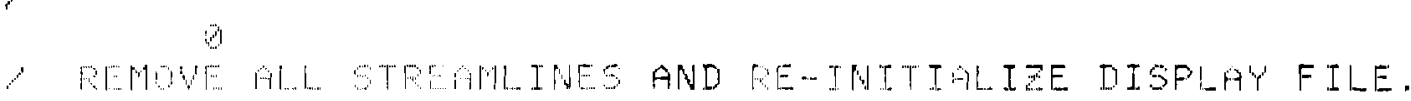




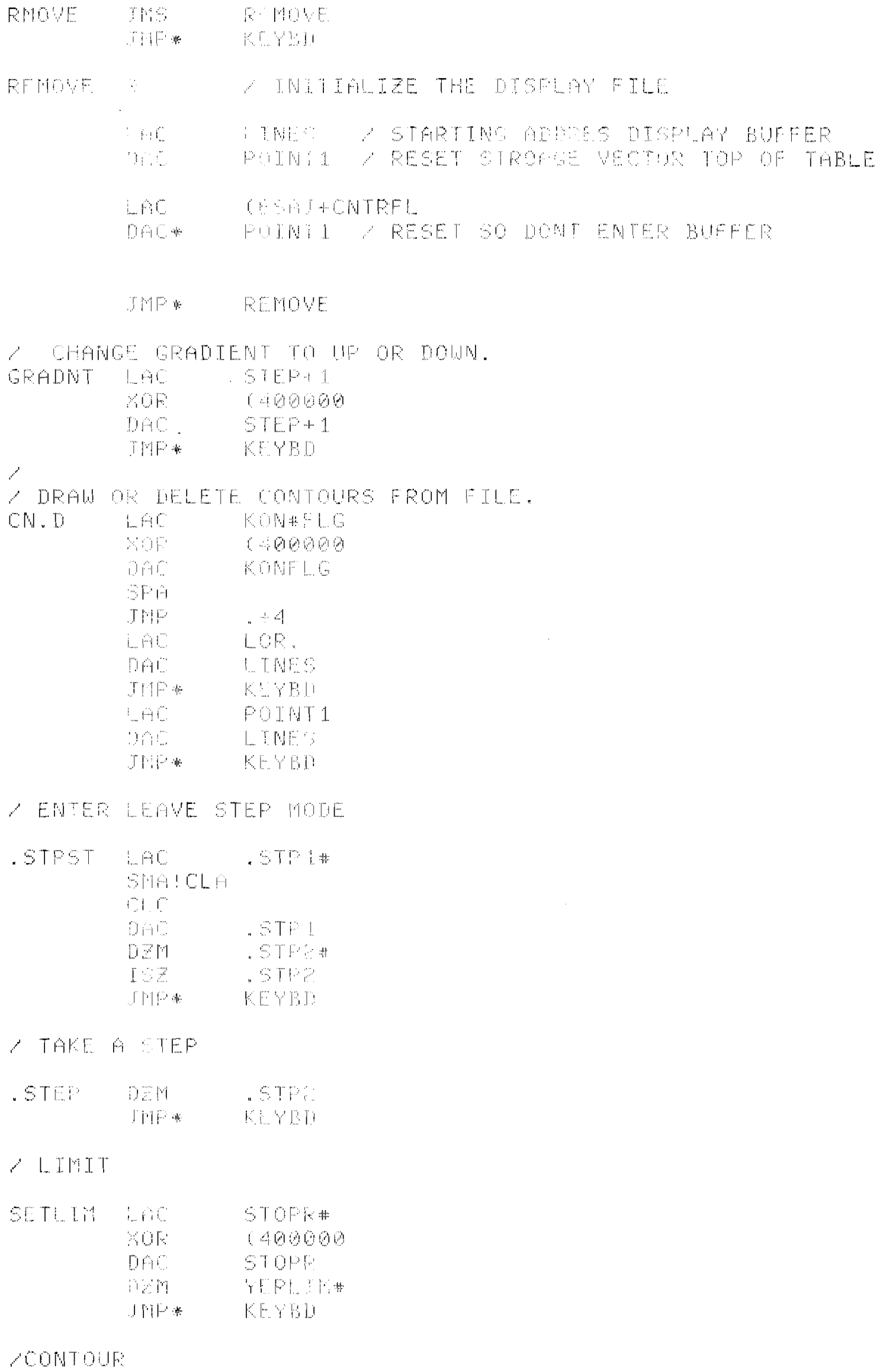




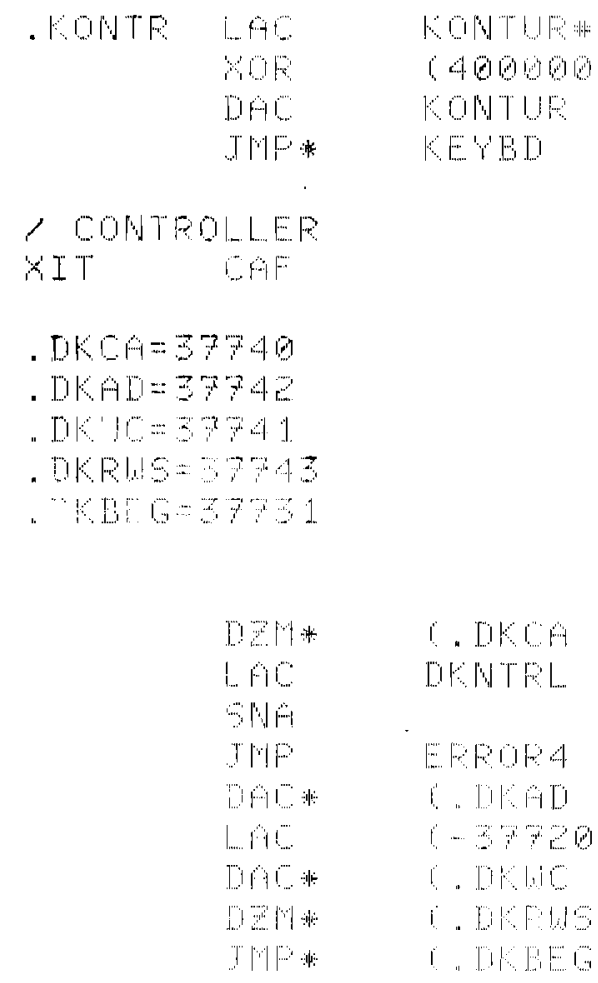

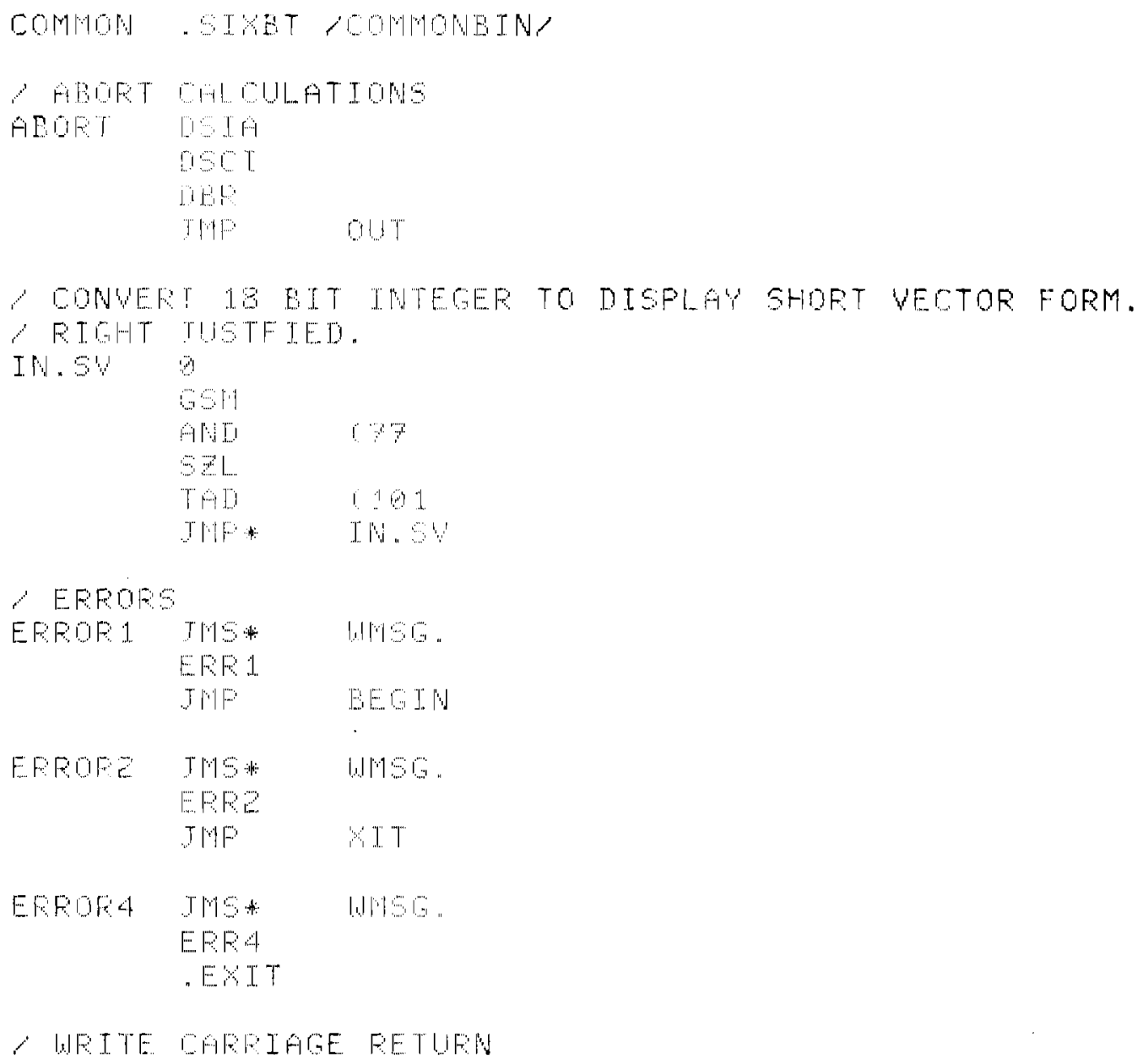




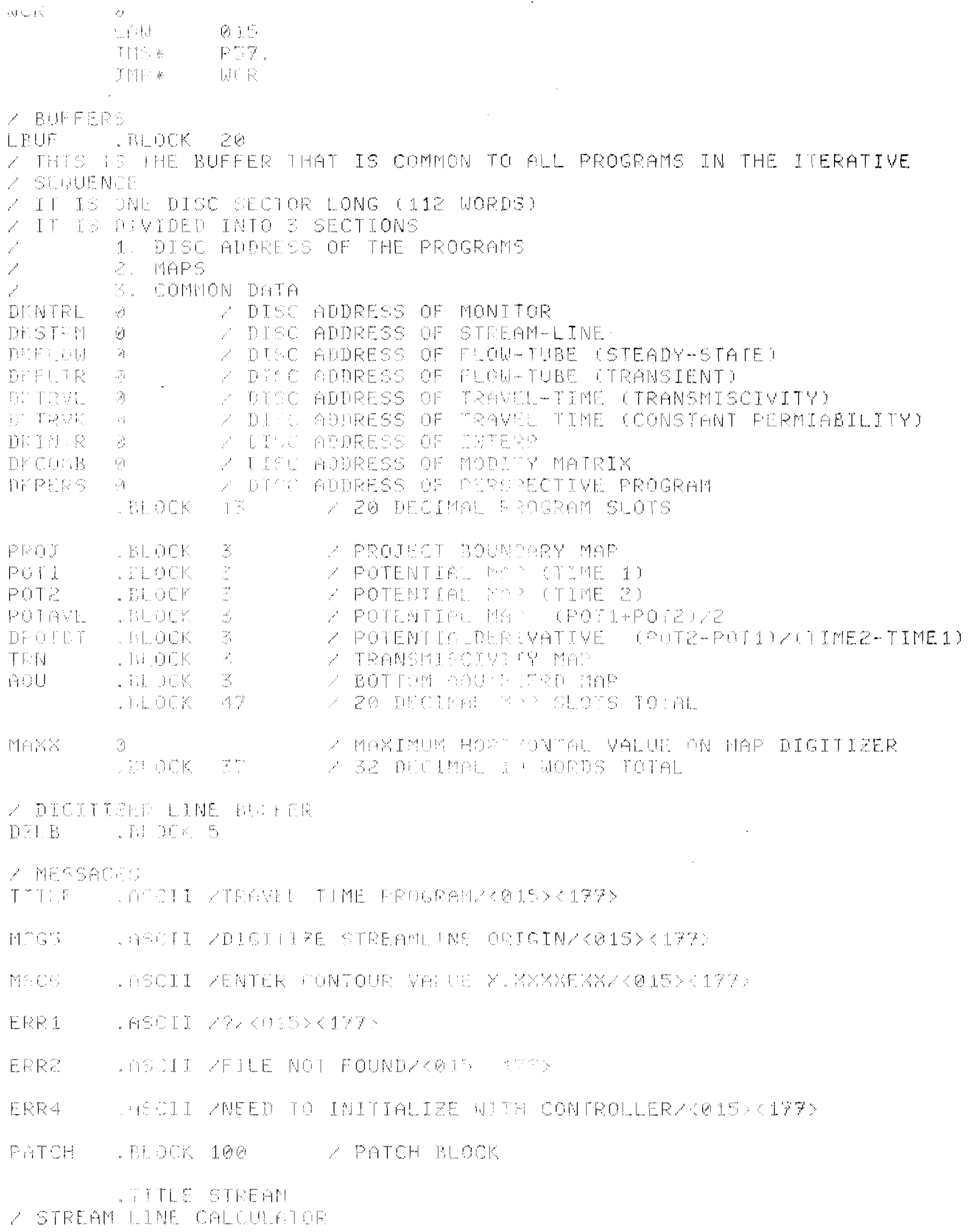




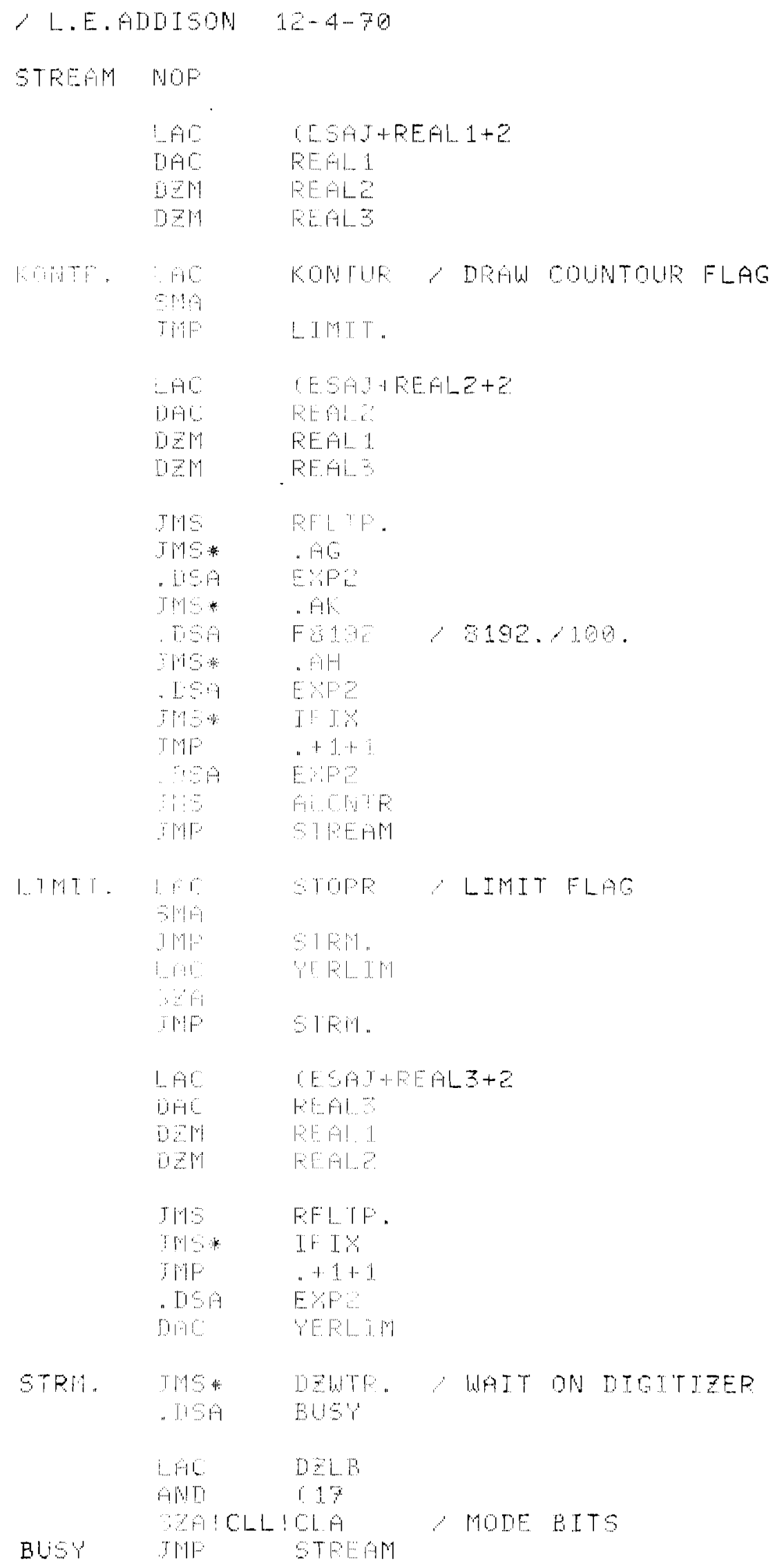




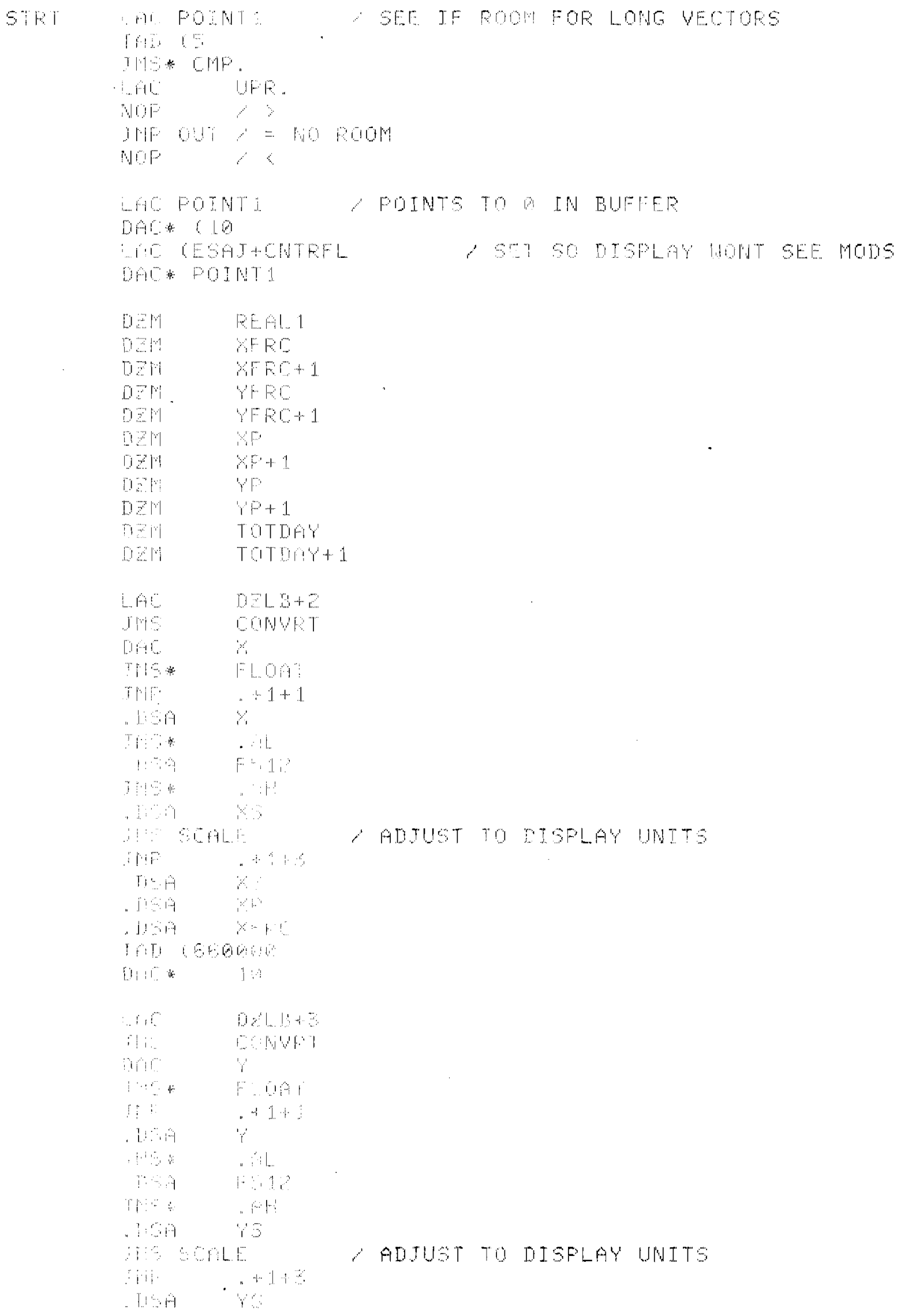

C -9 


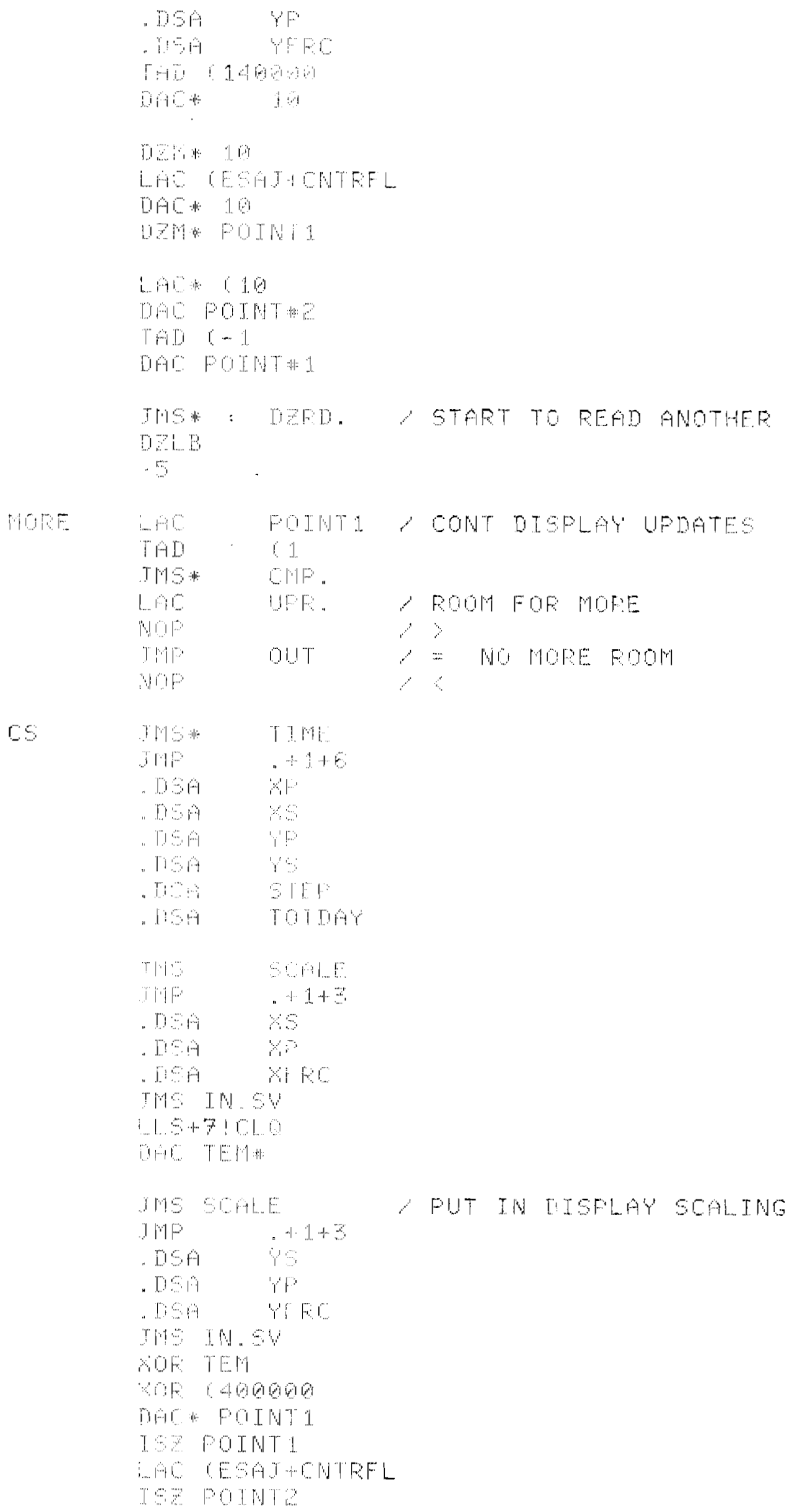




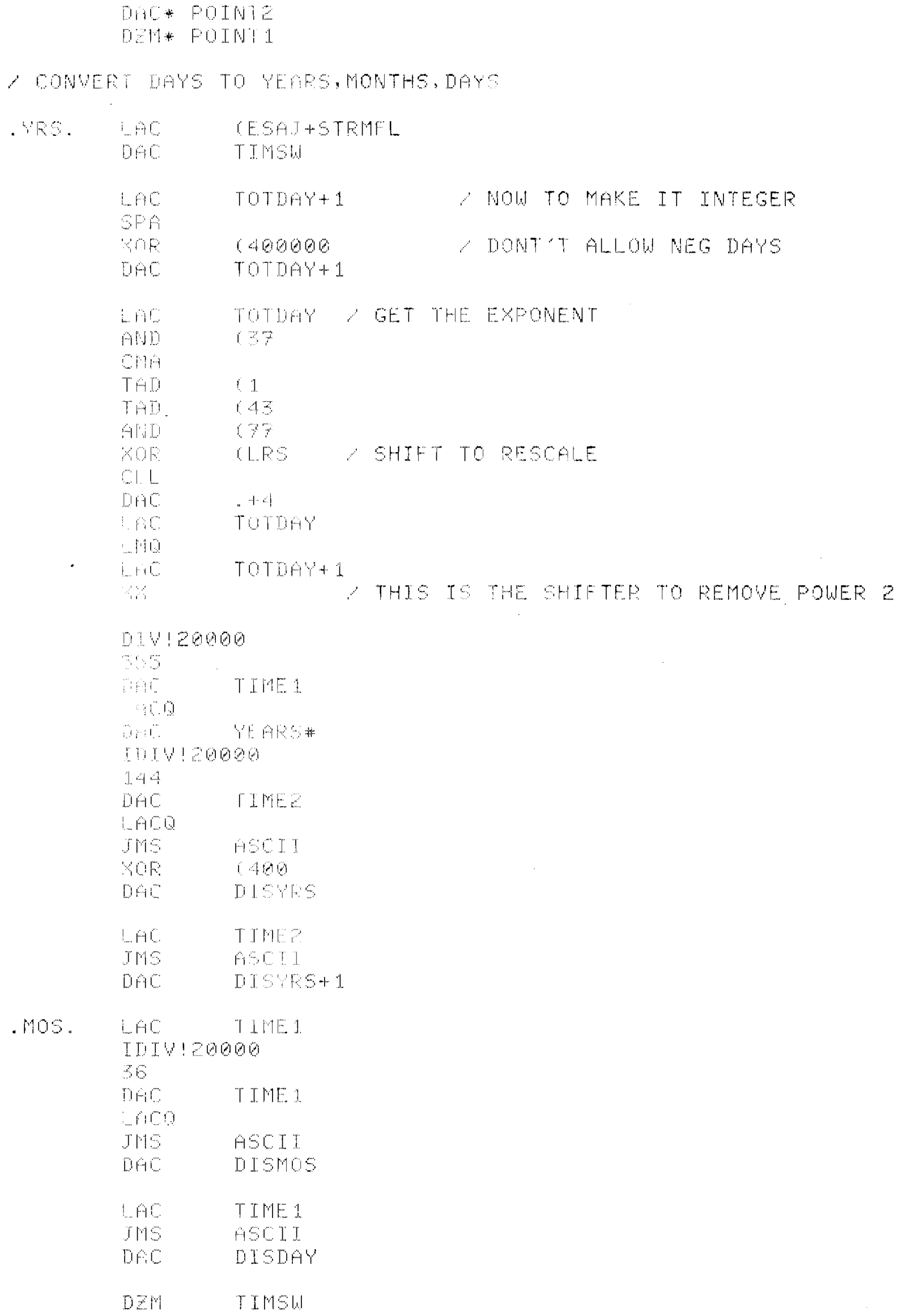




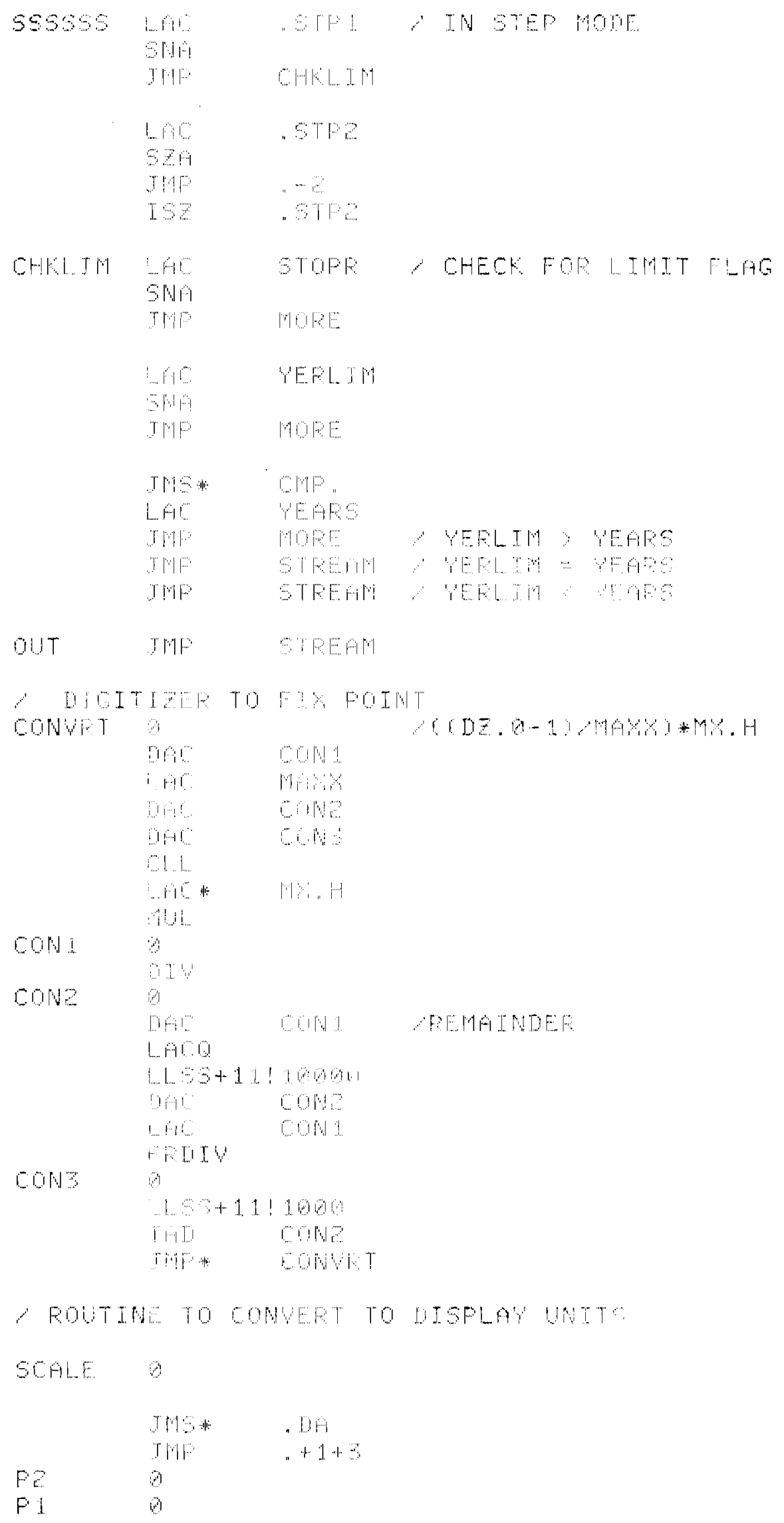




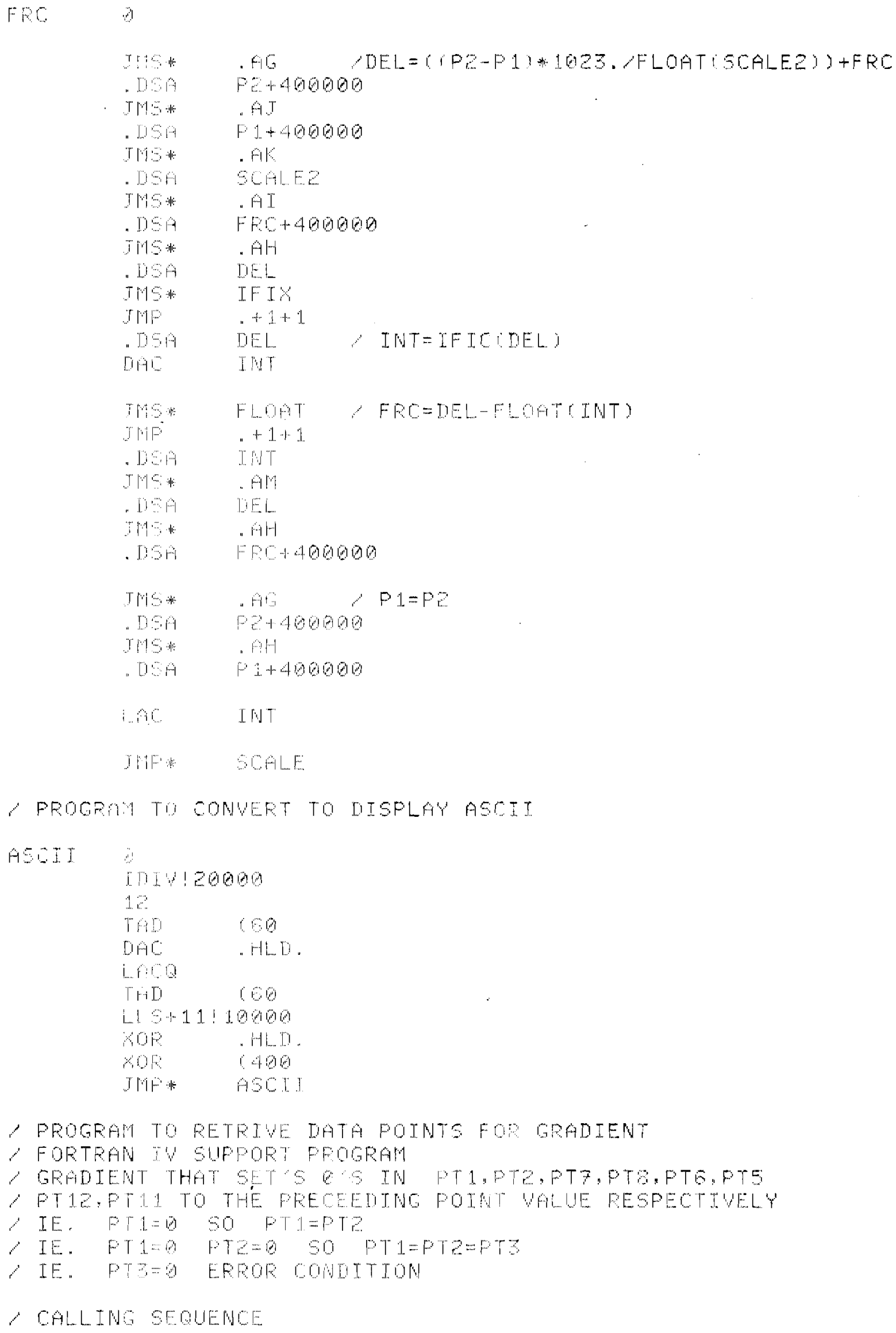




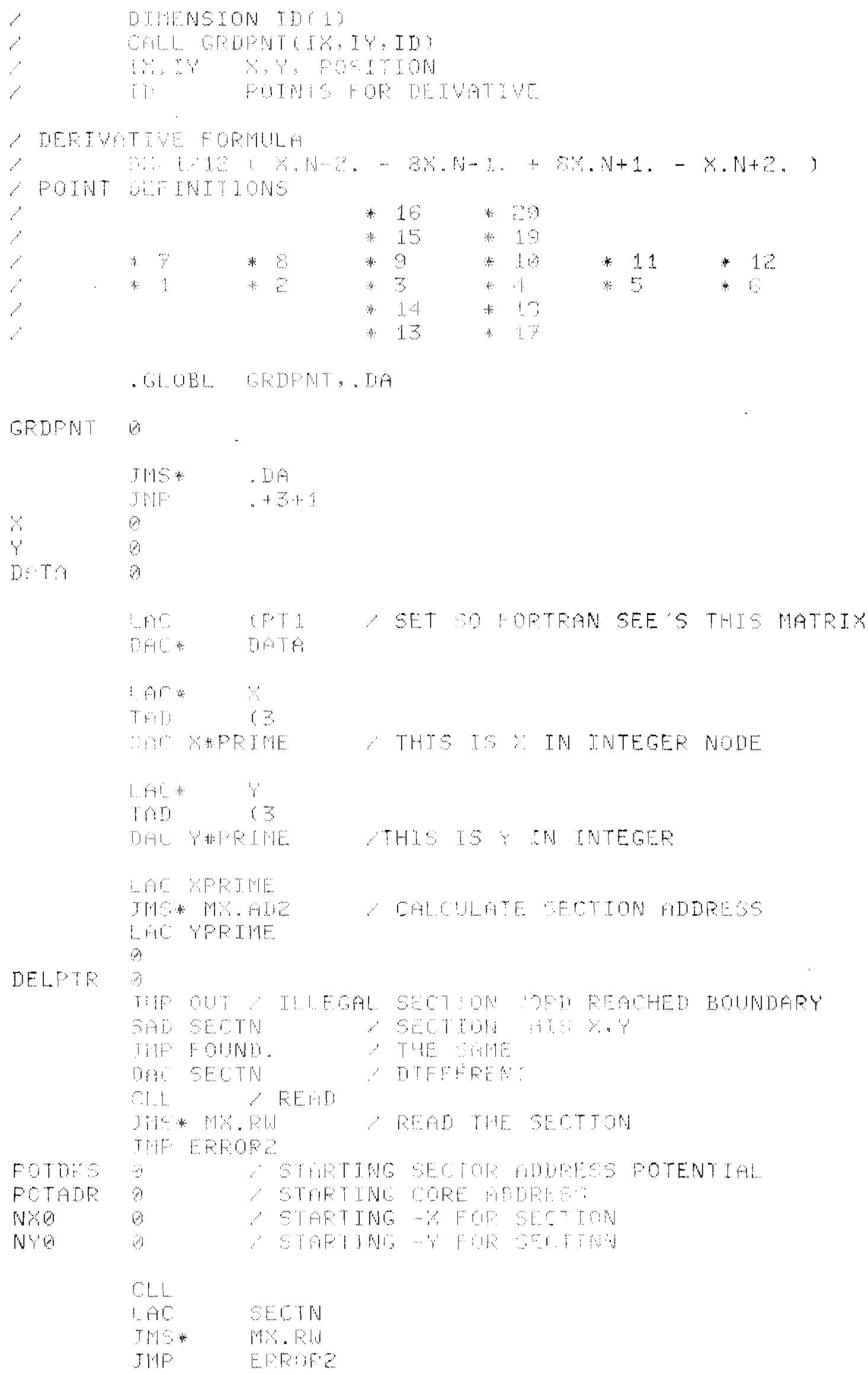




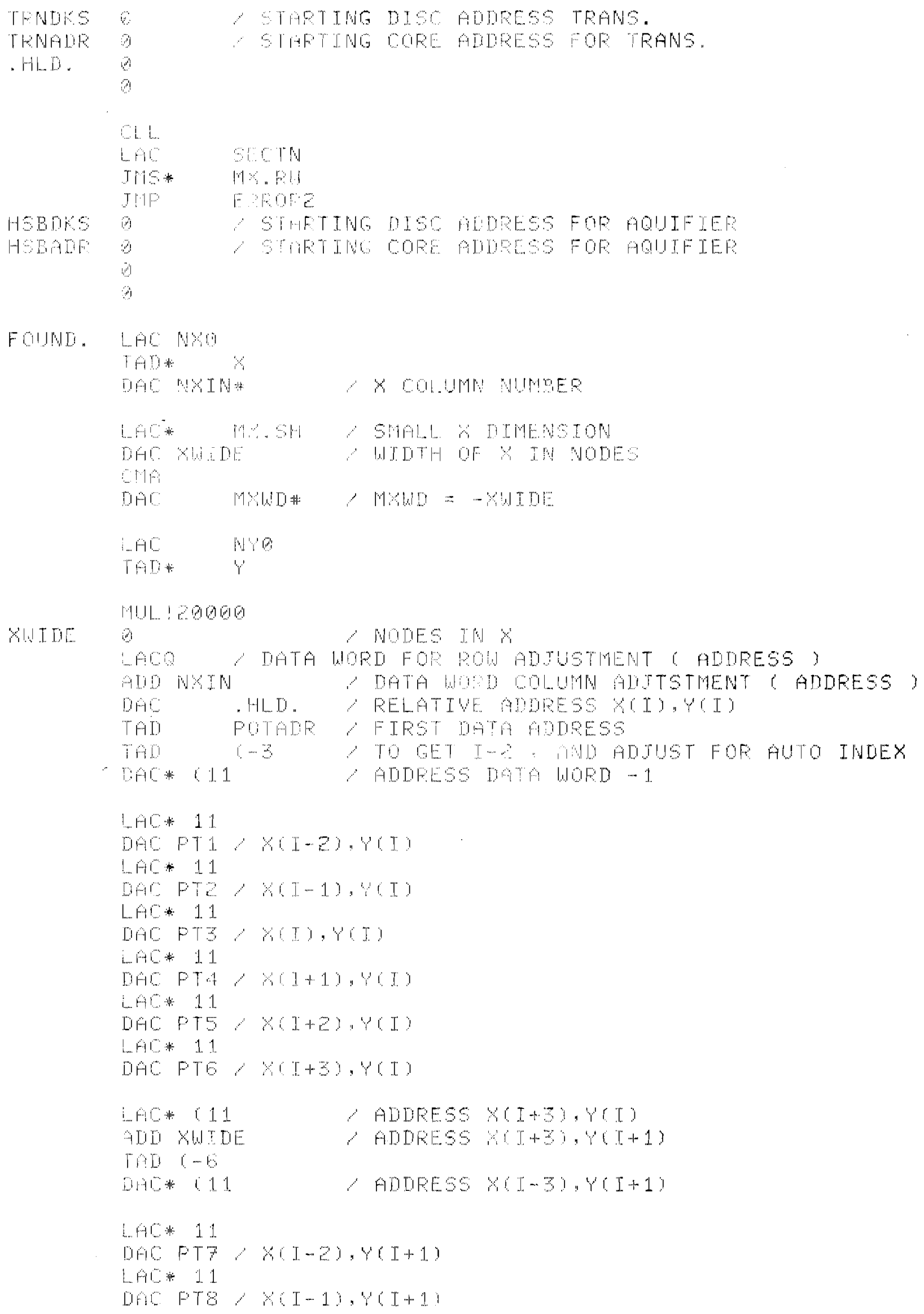




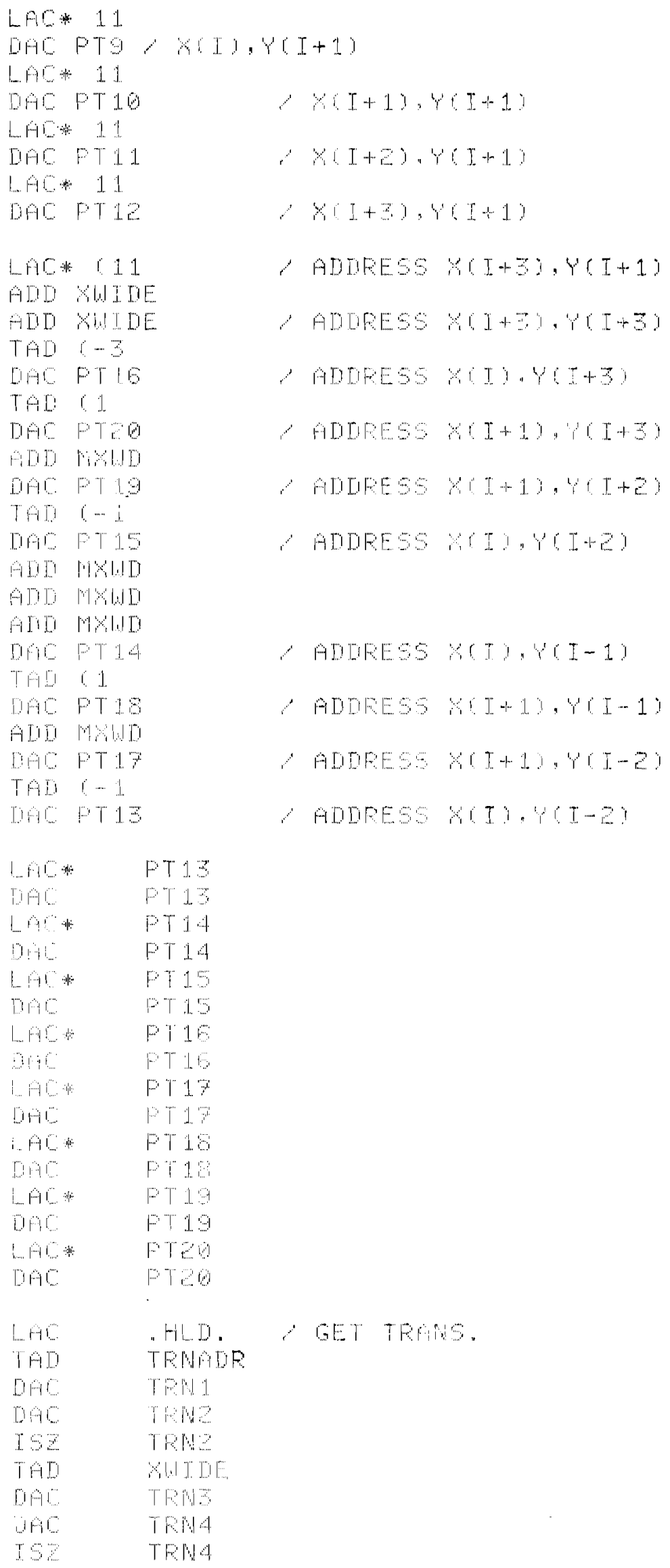




\begin{tabular}{|c|c|c|}
\hline$\angle A C H$ & TEN 1 & \\
\hline ШA世: & TEは & $\therefore T(X, I\}, Y I)\}$ \\
\hline 1.110 & Trate & \\
\hline 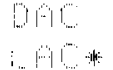 & $\begin{array}{l}\text { TFUE } \\
\text { TFAB }\end{array}$ & $4(Y) I+1), Y(I)$ \\
\hline М̈l. & TPMS & (T(S) I), Y (I+D) \\
\hline $\operatorname{LGO}$ & TEl & \\
\hline DAC & TPA & $\because T(K(I+1), Y(I+1))$ \\
\hline $\mathrm{AC}$ & 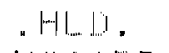 & $\triangle$ GET AQUFTER \\
\hline TAE & HSEALF & \\
\hline 190 & $1+\cdots 1$ & \\
\hline 0,0 & $H S E E$ & \\
\hline$I S m^{2}$ & H.SE & \\
\hline THI & $x \operatorname{xinE}$ & \\
\hline $30 \mathrm{C}$ & $\mathrm{HSBS}$ & \\
\hline Dis: & $\mid-1,5$ & \\
\hline $\mathrm{is}$ & $H=8.4$ & \\
\hline$\therefore 95$ & HE 1 & \\
\hline 19 & 401 & $\because H\left(X^{\prime}(I), Y(I)\right.$ \\
\hline 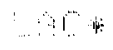 & $1.5,13$ & \\
\hline Dीि & $45 \mathrm{Ez}$ & $(H C K(I+1): Y(I))$ \\
\hline 10 & $15 \mathrm{ax}$ & \\
\hline Uh & $H \mathrm{~B}$ & $(H(x), Y, Y(T+1)$ \\
\hline 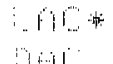 & HSE+ & $\therefore H(X 0 T+1), 0 I+1)$ \\
\hline & & $=A(B)+1): Y(1+$ \\
\hline
\end{tabular}

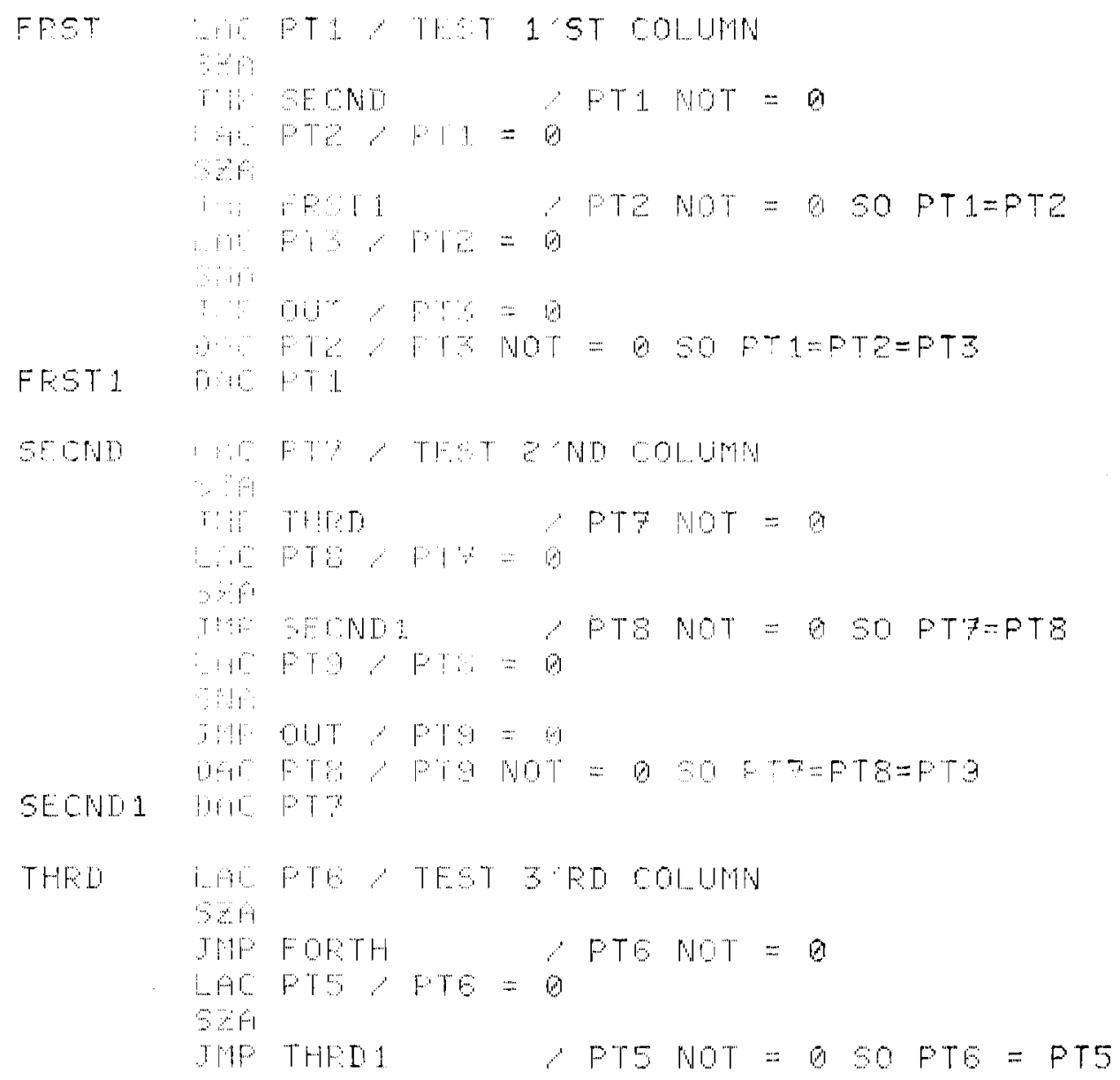




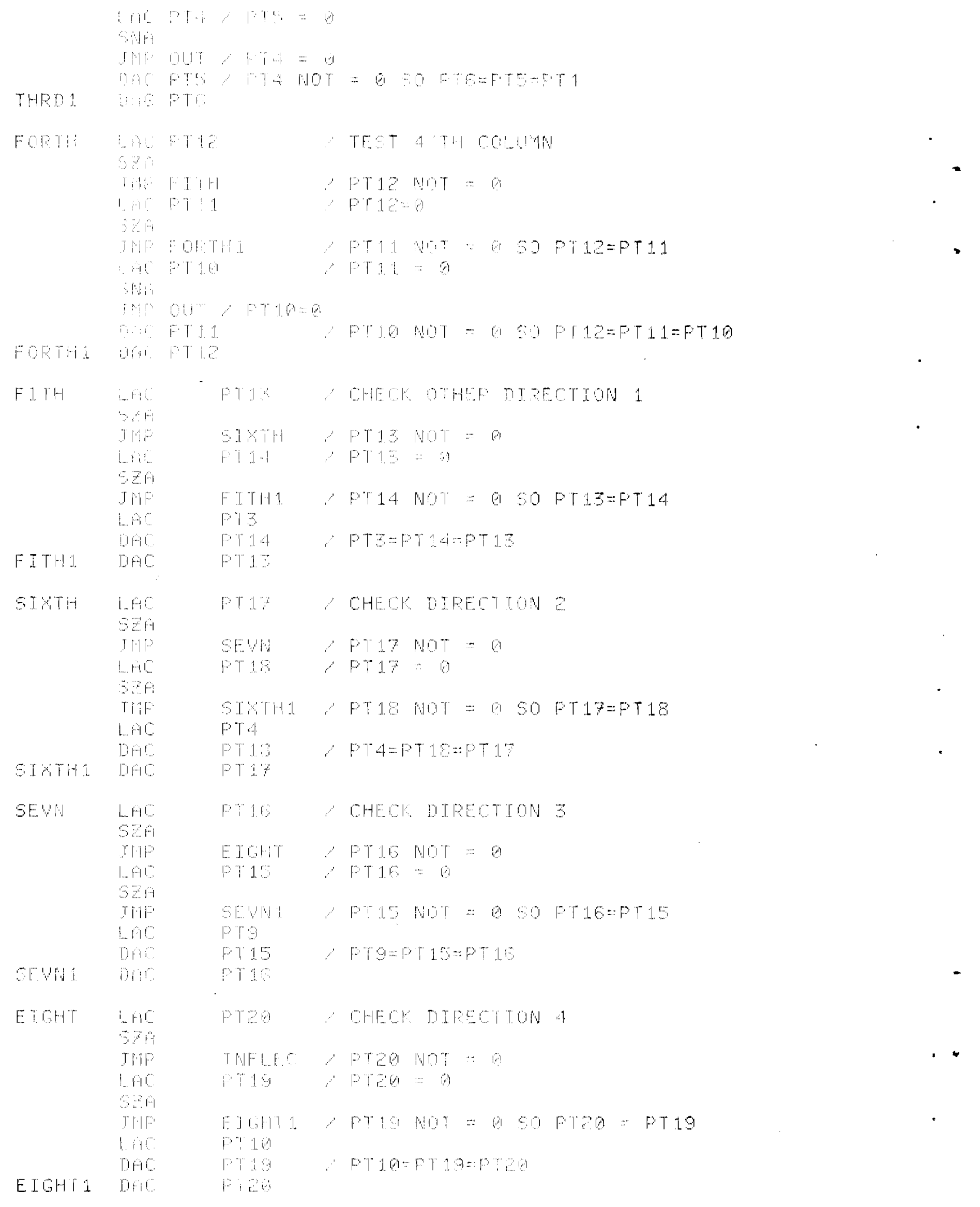




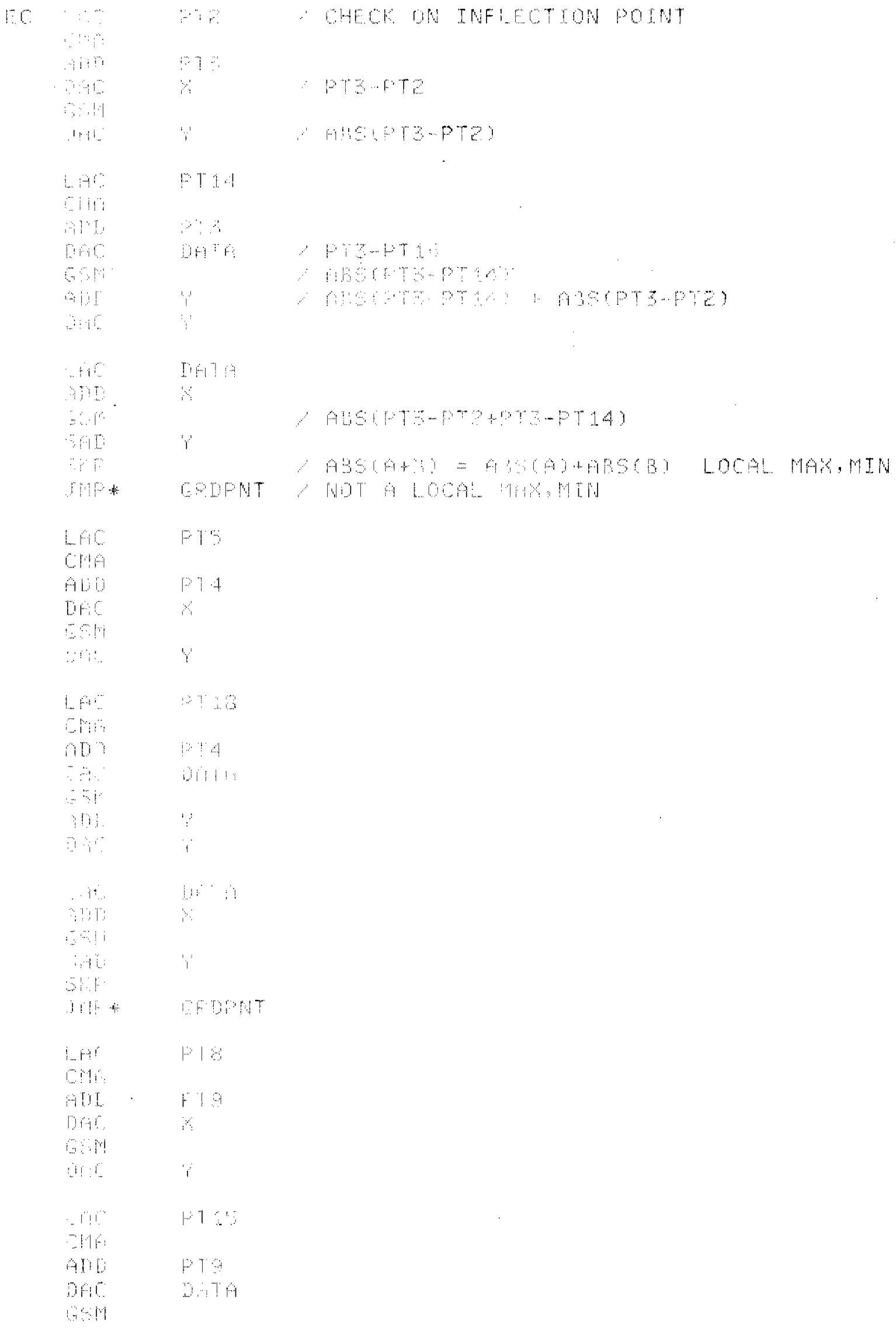

C- 19 


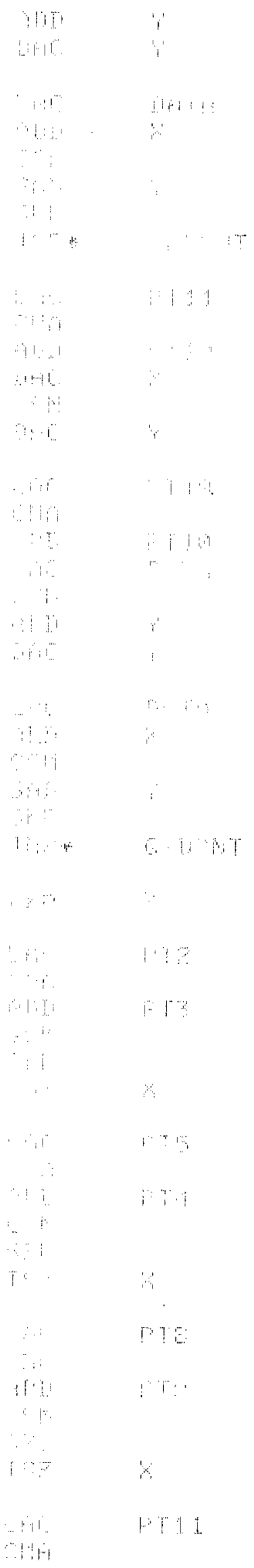




\begin{tabular}{|c|c|}
\hline $\begin{array}{r}41 \\
611 \\
15 z\end{array}$ & PT 10 \\
\hline ( & $y$ \\
\hline 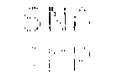 & oul \\
\hline$s+p$ & 4 \\
\hline Sint & GPOPNT \\
\hline$m$ & sis \\
\hline $\begin{array}{l}80 \\
119\end{array}$ & $P T 14$ \\
\hline $\begin{array}{c}9 \pi i \\
6 \% \\
9 \%\end{array}$ & FT3 \\
\hline $15 z$ & 8 \\
\hline ifo & PT 18 \\
\hline $\begin{array}{l}6 \pm[ \\
5.4 \\
54\end{array}$ & FTA \\
\hline$I 0 Z$ & $x$ \\
\hline $\begin{array}{l}40 \\
6 ! A\end{array}$ & PTIS \\
\hline $\begin{array}{l}81 ! \\
8.1 !\end{array}$ & Prs \\
\hline $1:$ & 8 \\
\hline $\begin{array}{l}9 \mathrm{C} \\
\mathrm{9H}\end{array}$ & $P 719$ \\
\hline $\begin{array}{l}511 \\
9 \% 1 \\
9 \% 1\end{array}$ & P19 \\
\hline 16 & $\mathrm{x}$ \\
\hline$\therefore A 1$ & $\because$ \\
\hline$r: !$ & 001 \\
\hline 30 & 14 \\
\hline 1011 & $01 T$ \\
\hline$y=*$ & GADONT \\
\hline
\end{tabular}

PT1
PTE
PTB
PTA
PTS
PTE
PTY
PTE




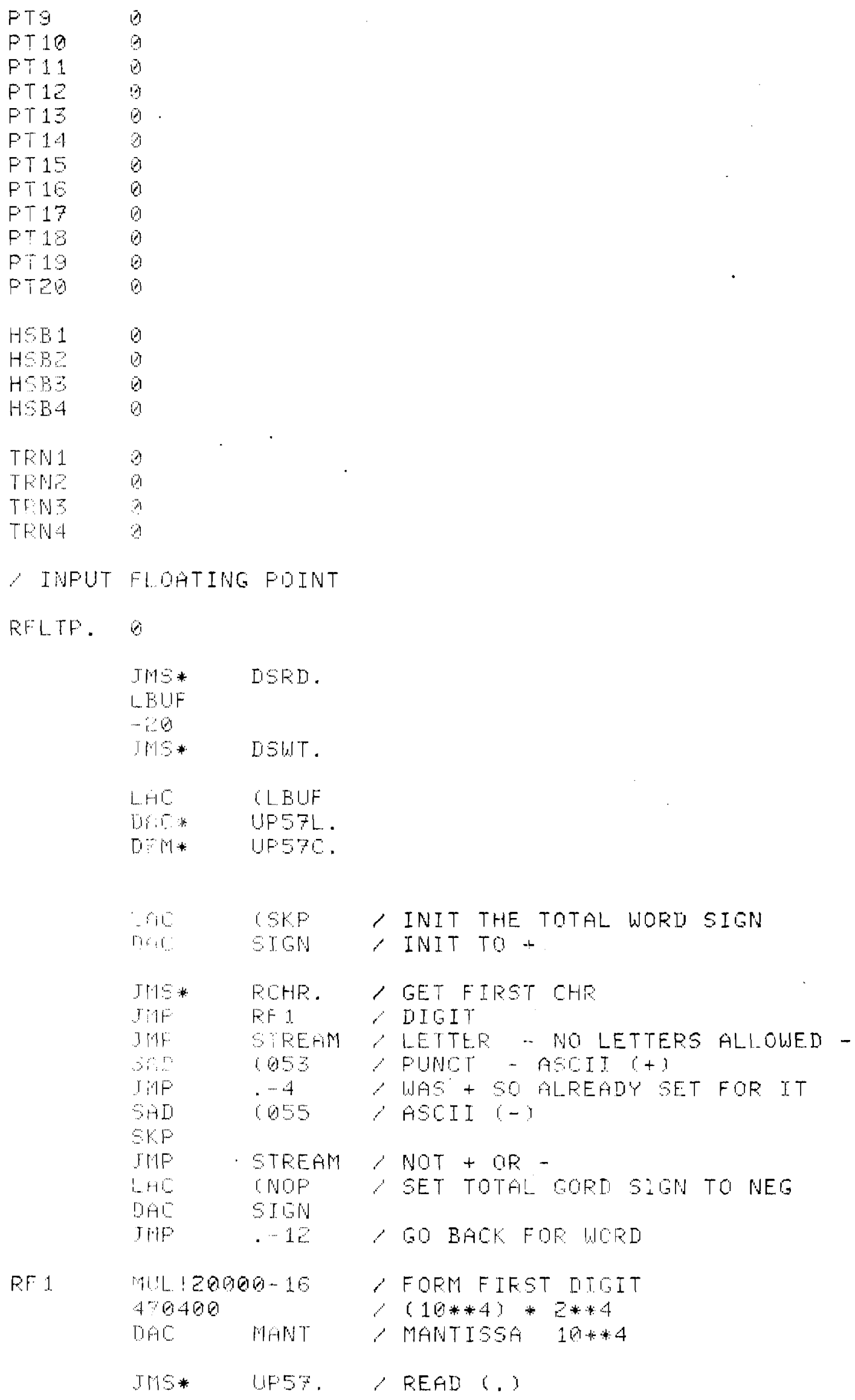




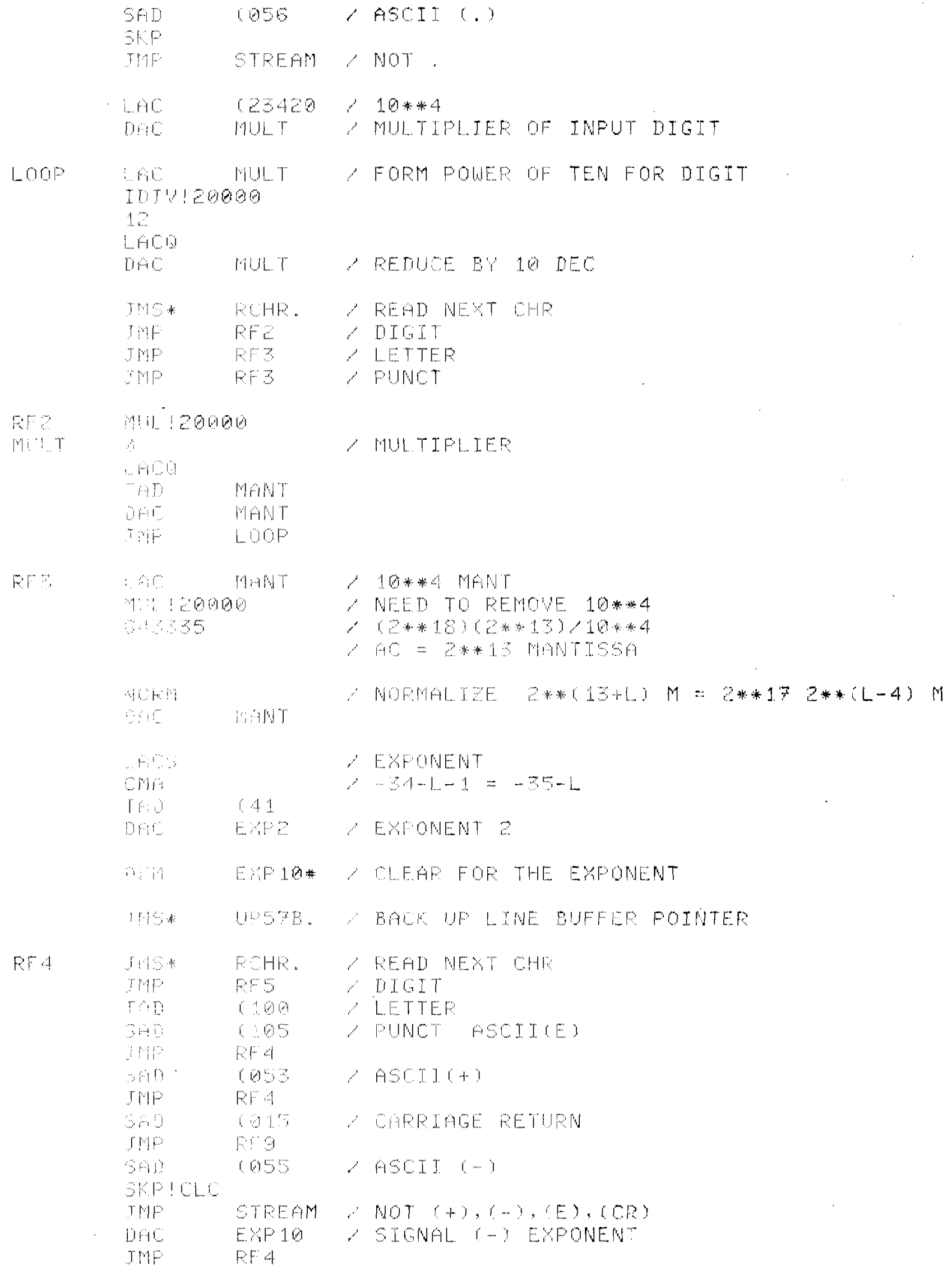




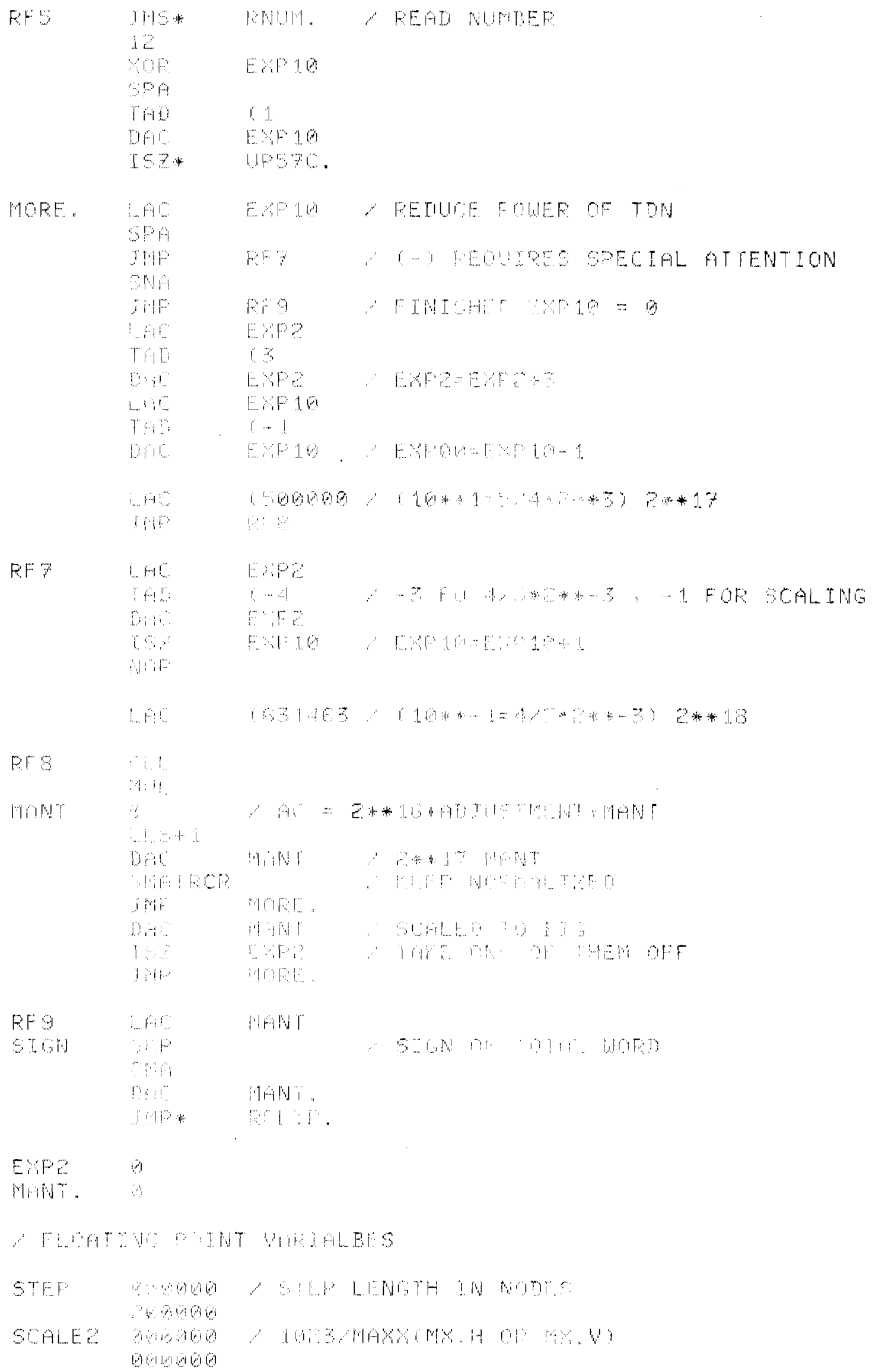




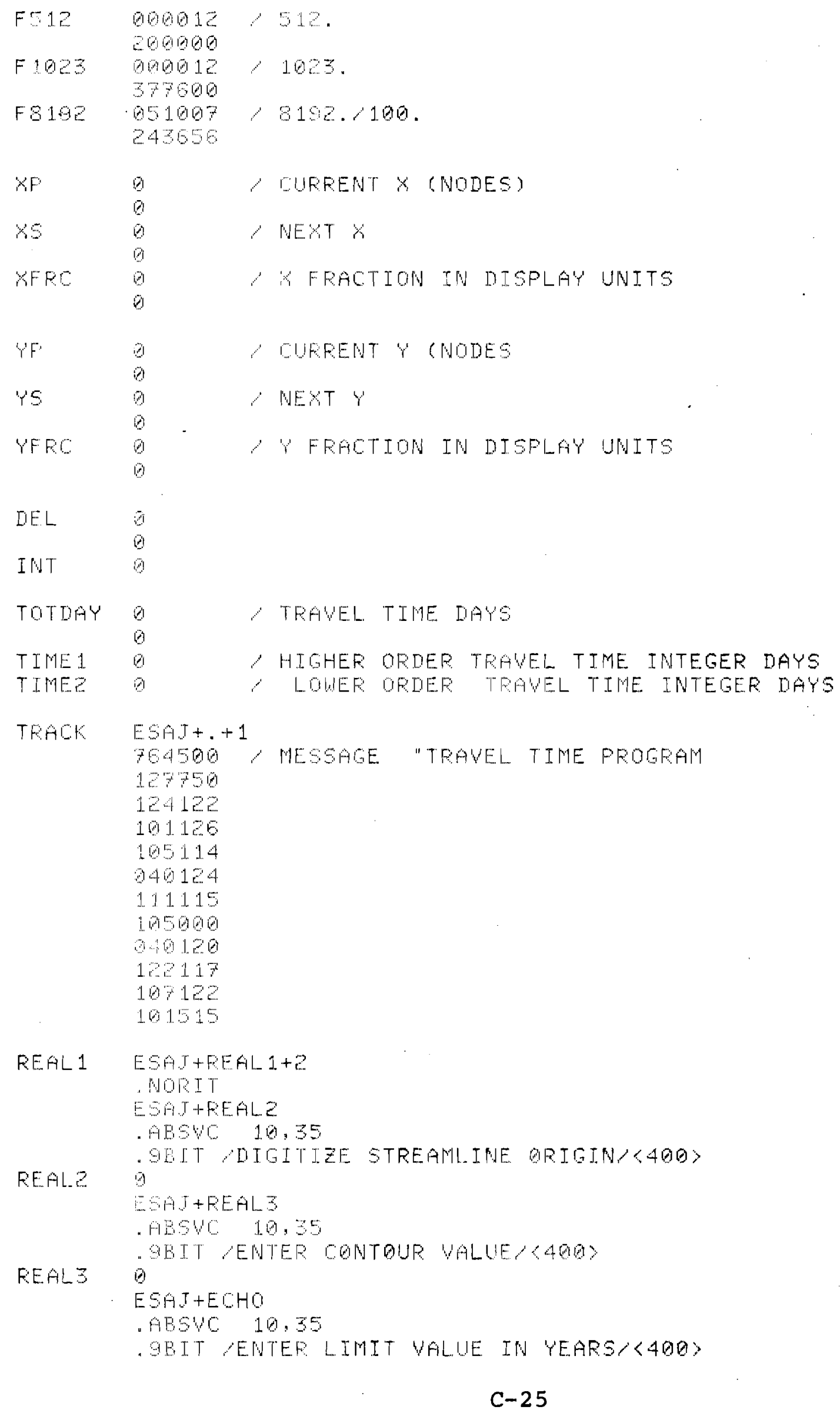




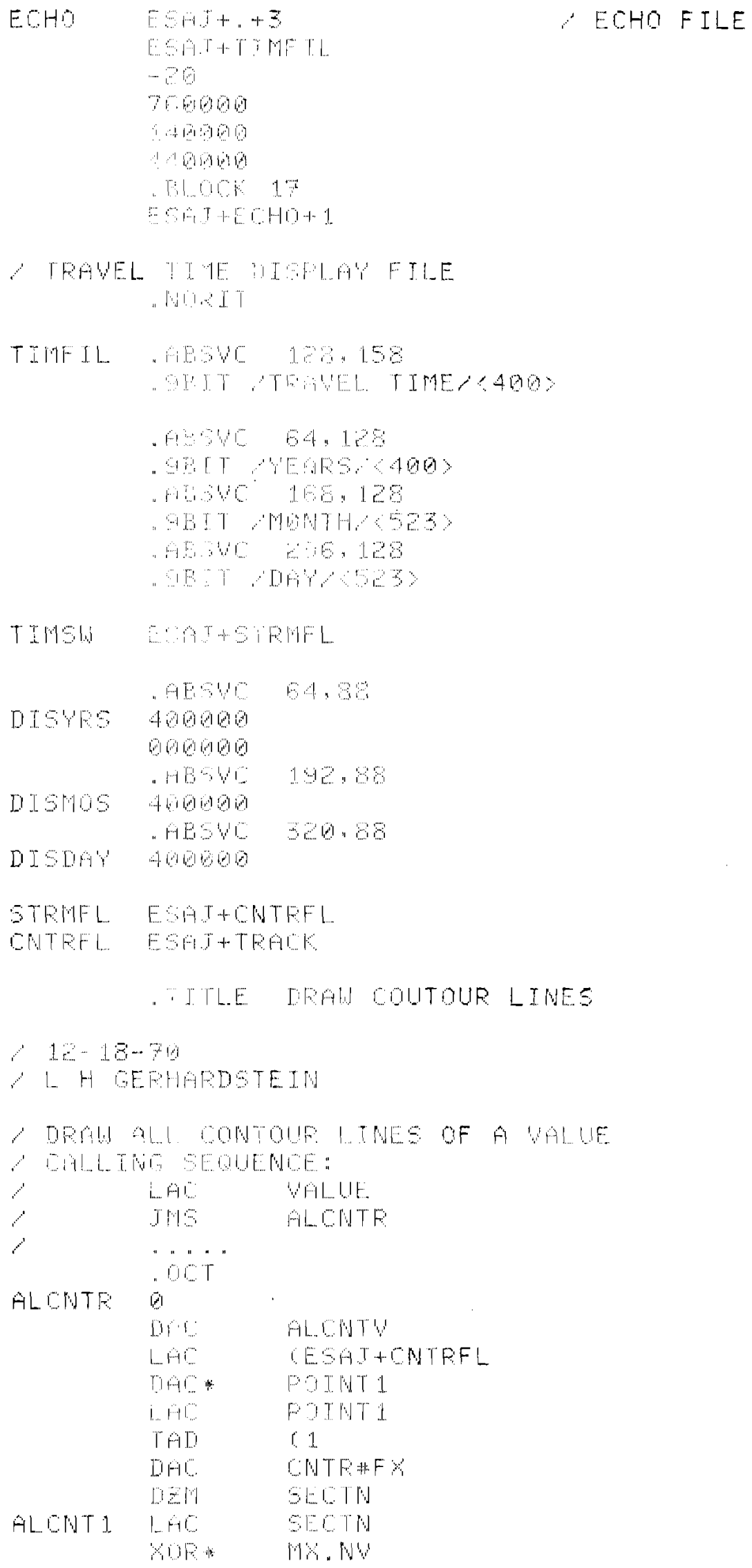




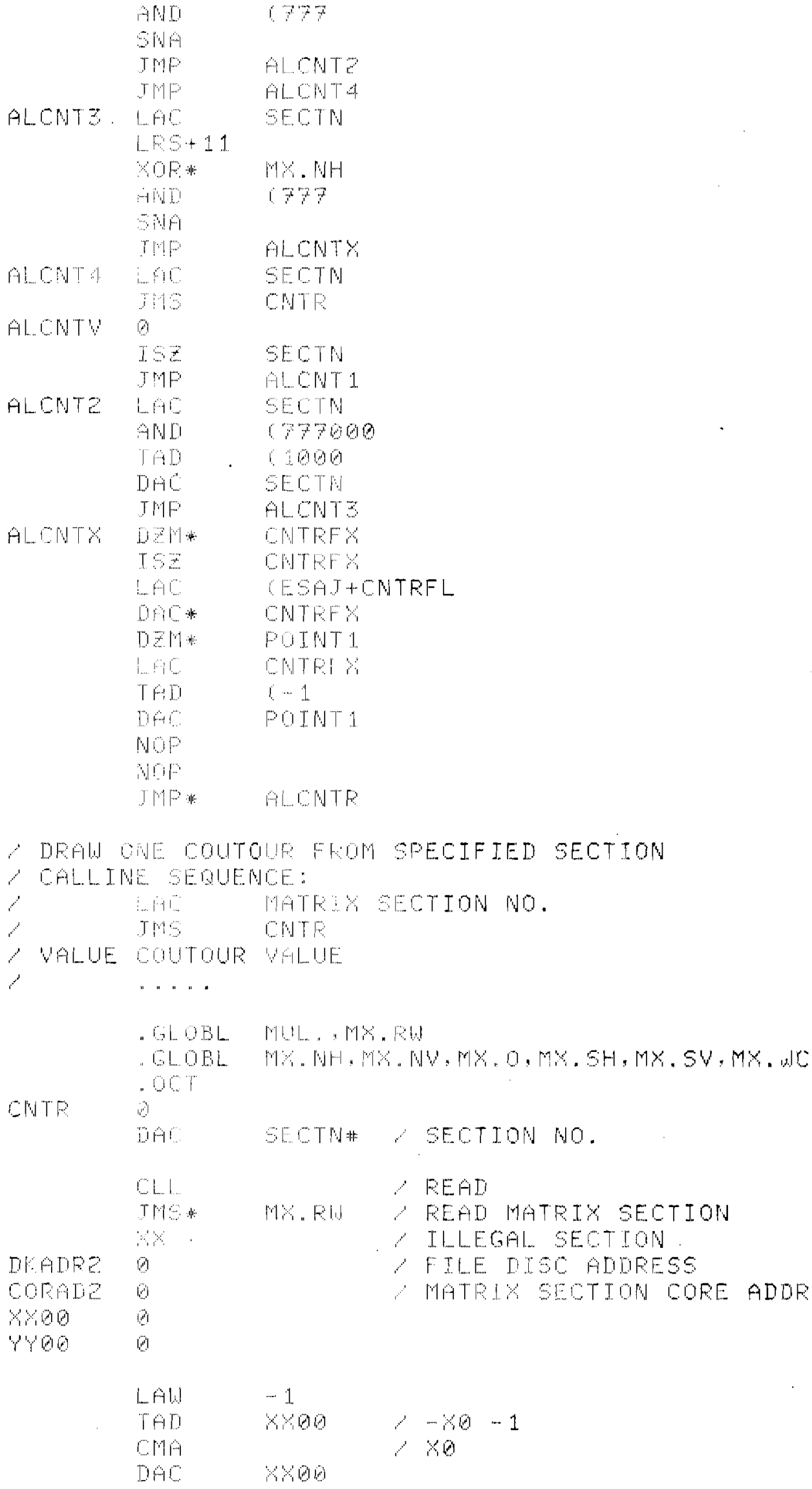




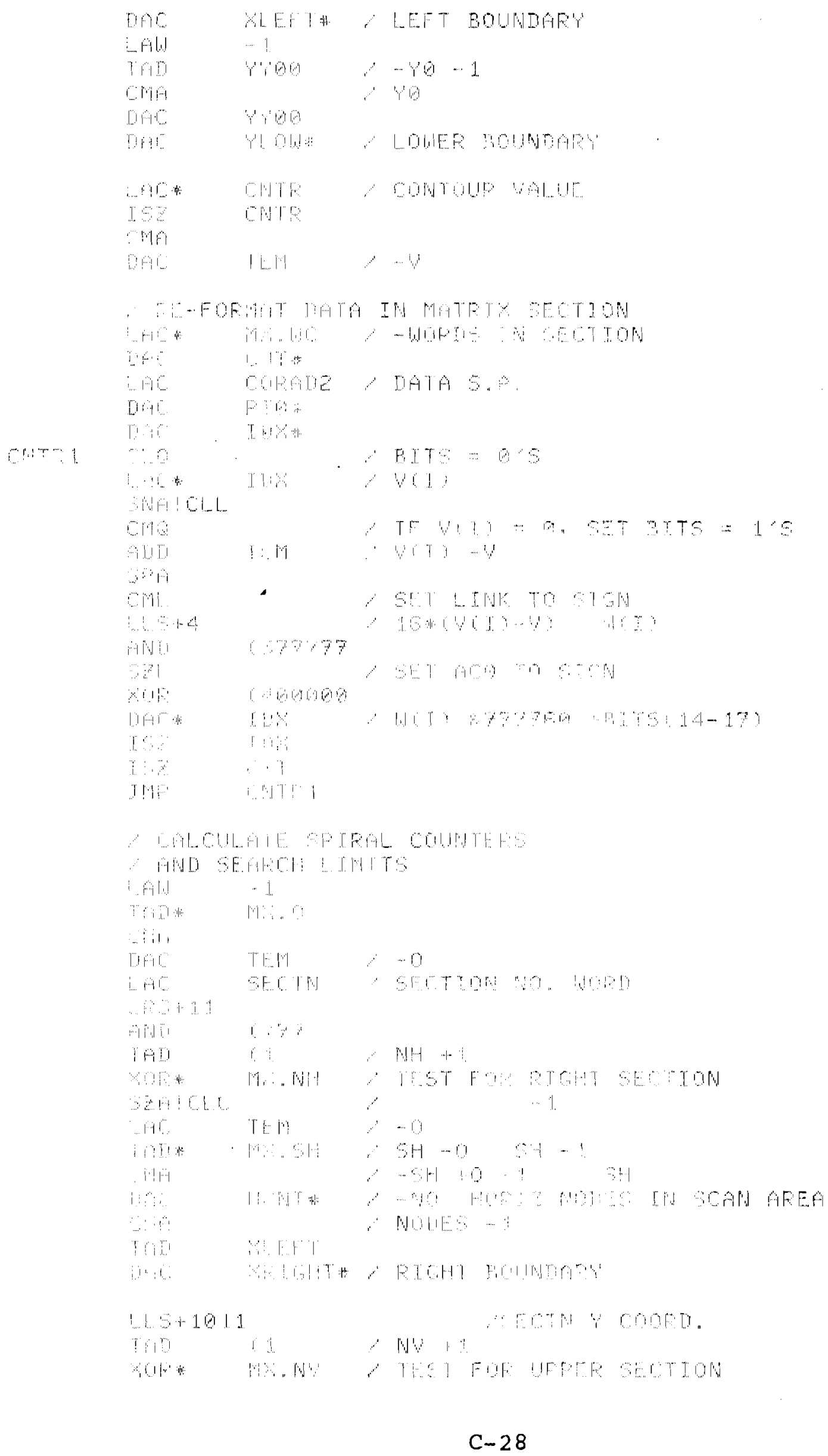




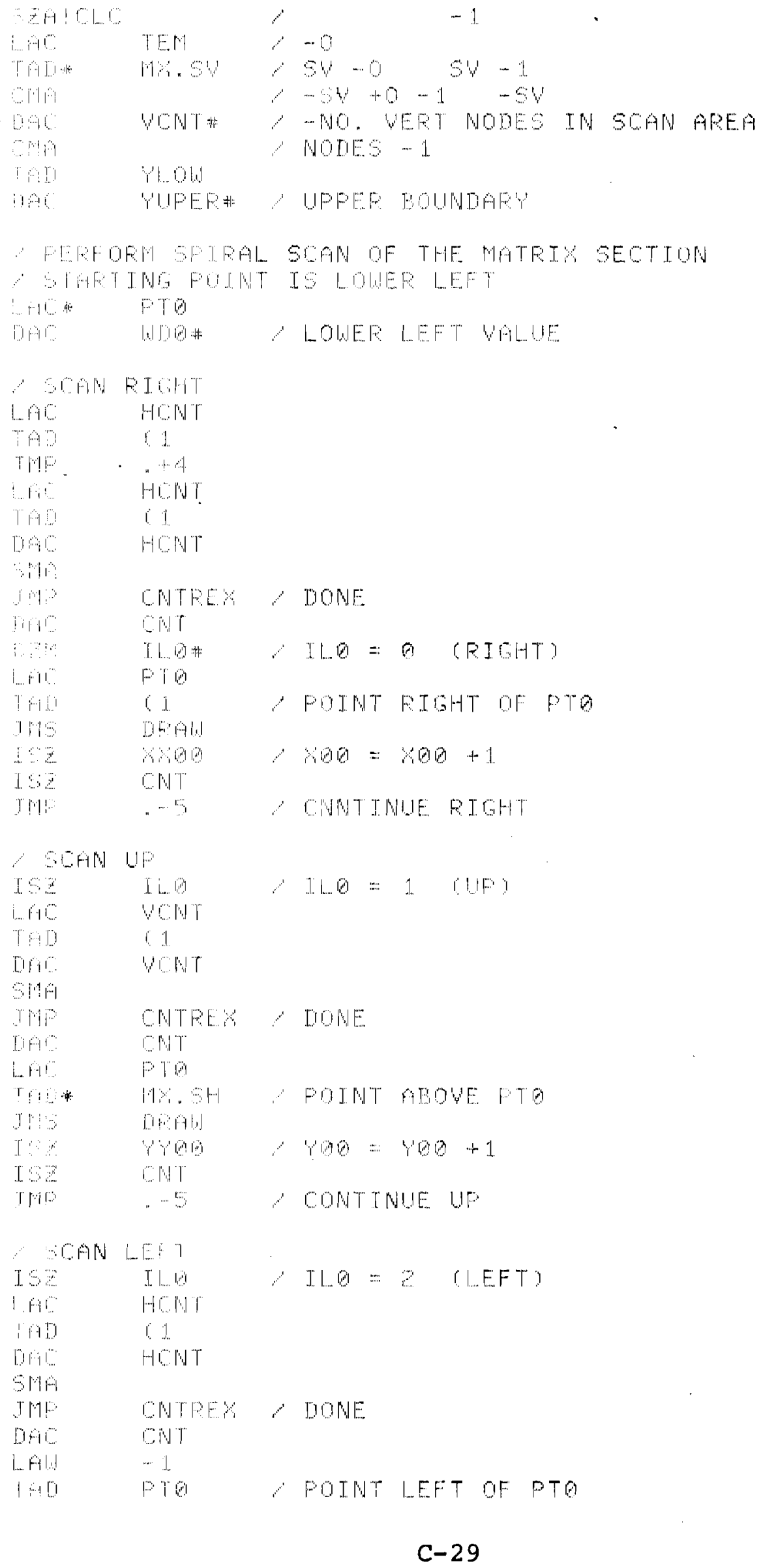




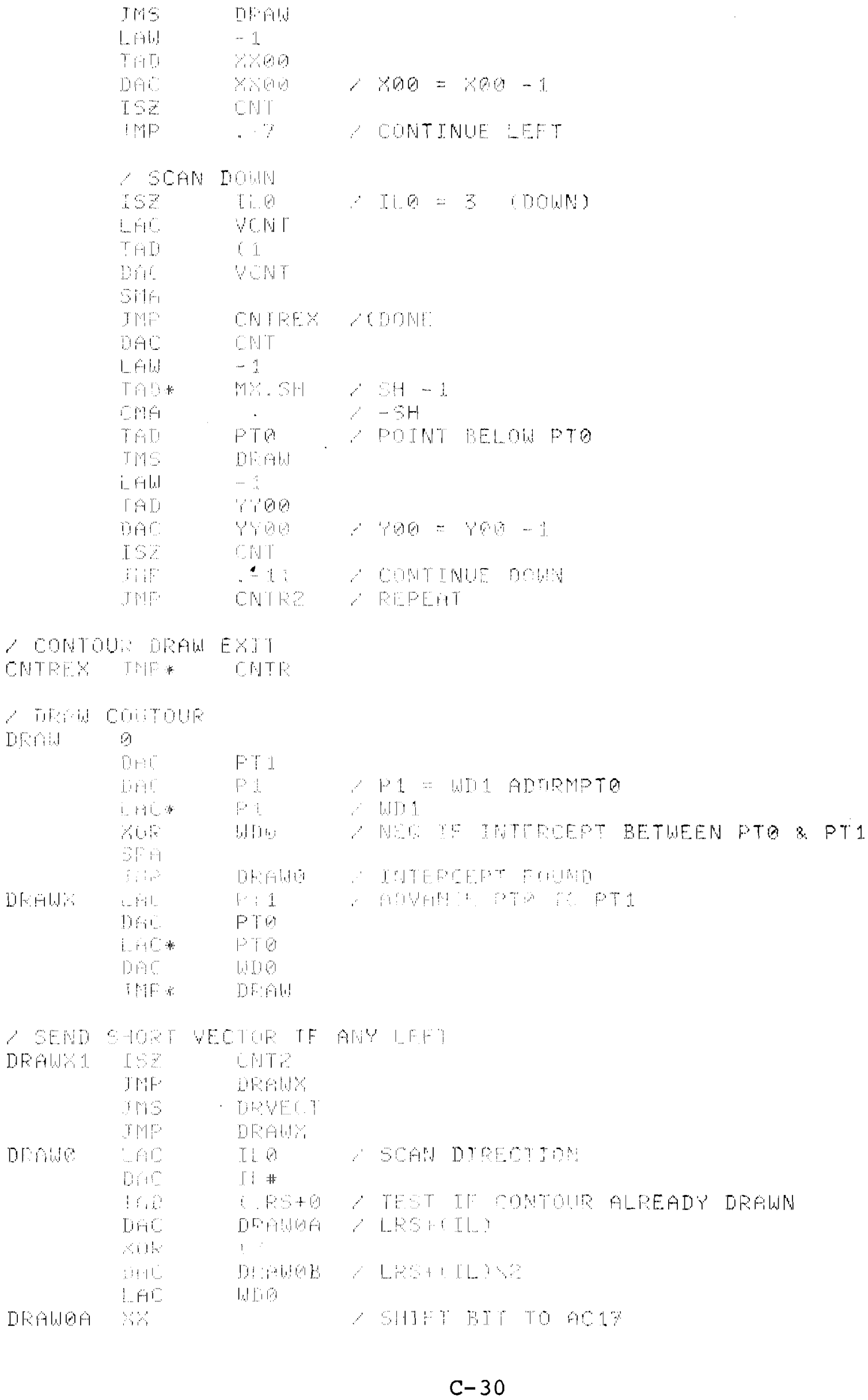




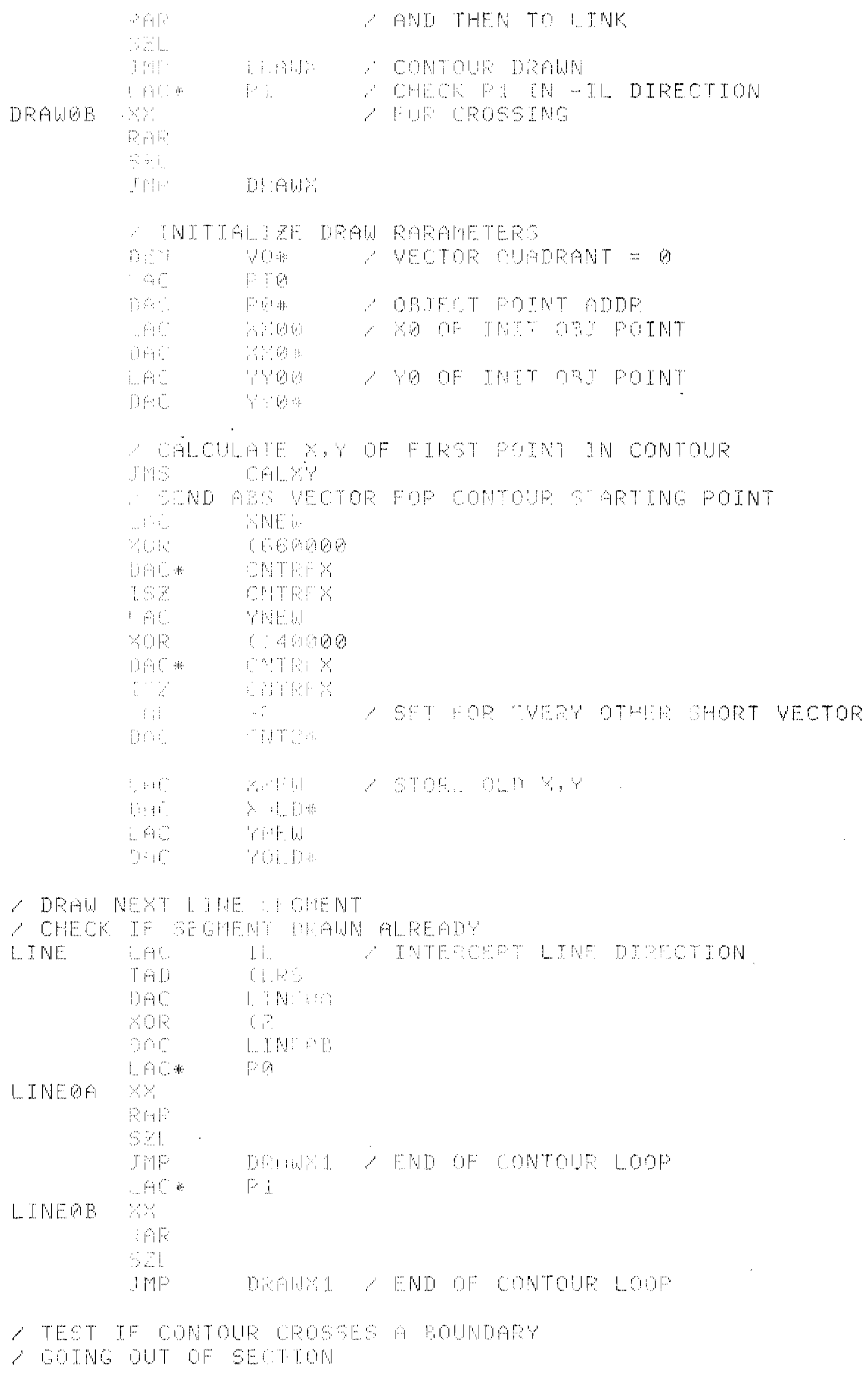




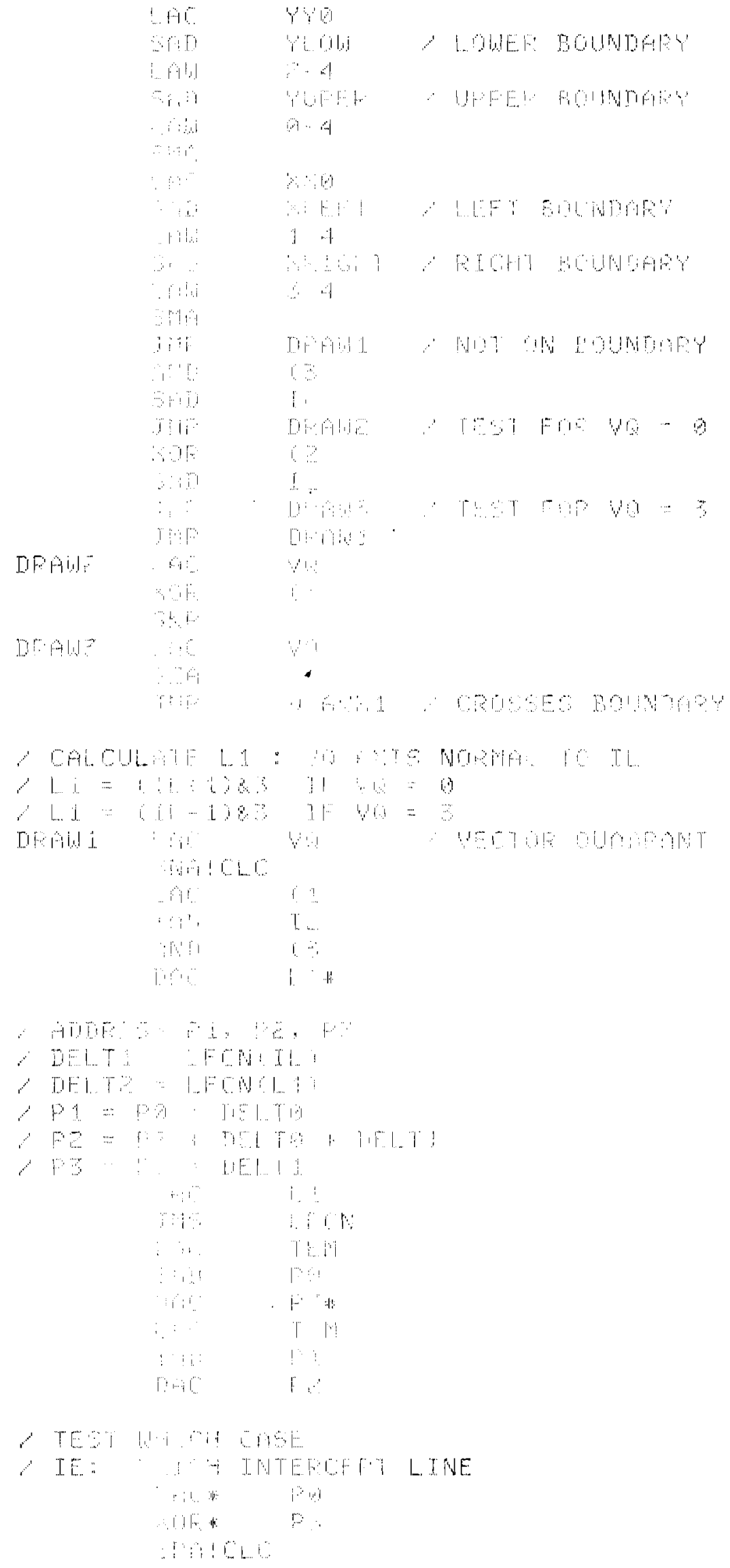




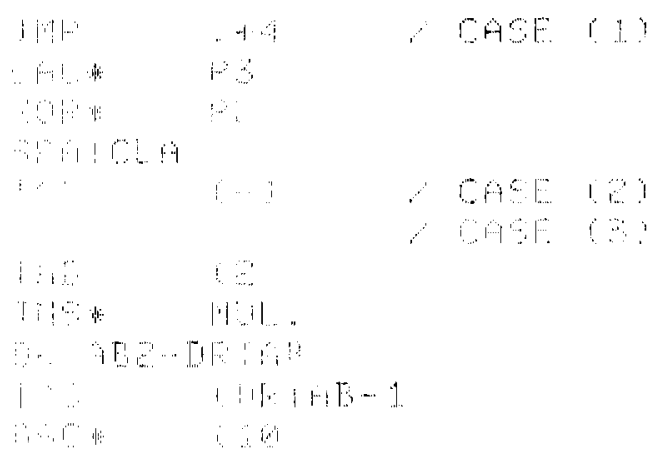

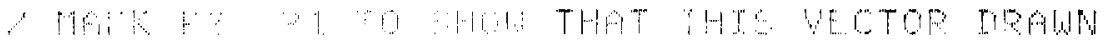

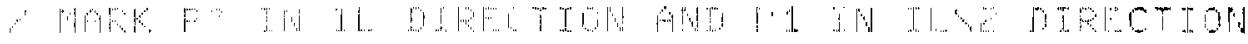
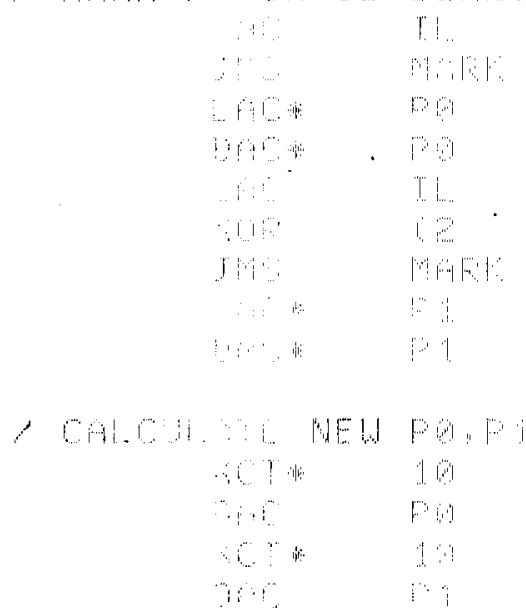

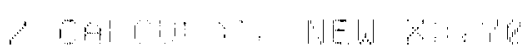

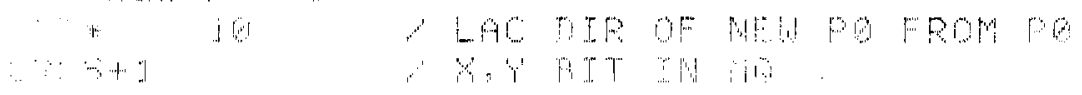

$\therefore$

1..stind 1

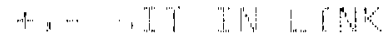

83

iाi

$\therefore=$ $\therefore 93 \mathrm{~T}$

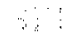

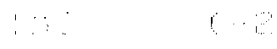

119

110

il

$\therefore$ and

7

$1 \%$ in

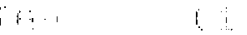

if

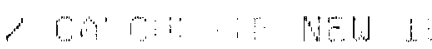

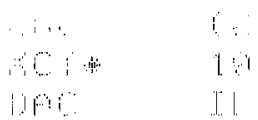

CALCUL I MEN M $\begin{array}{lll}-10 & i\end{array}$ 


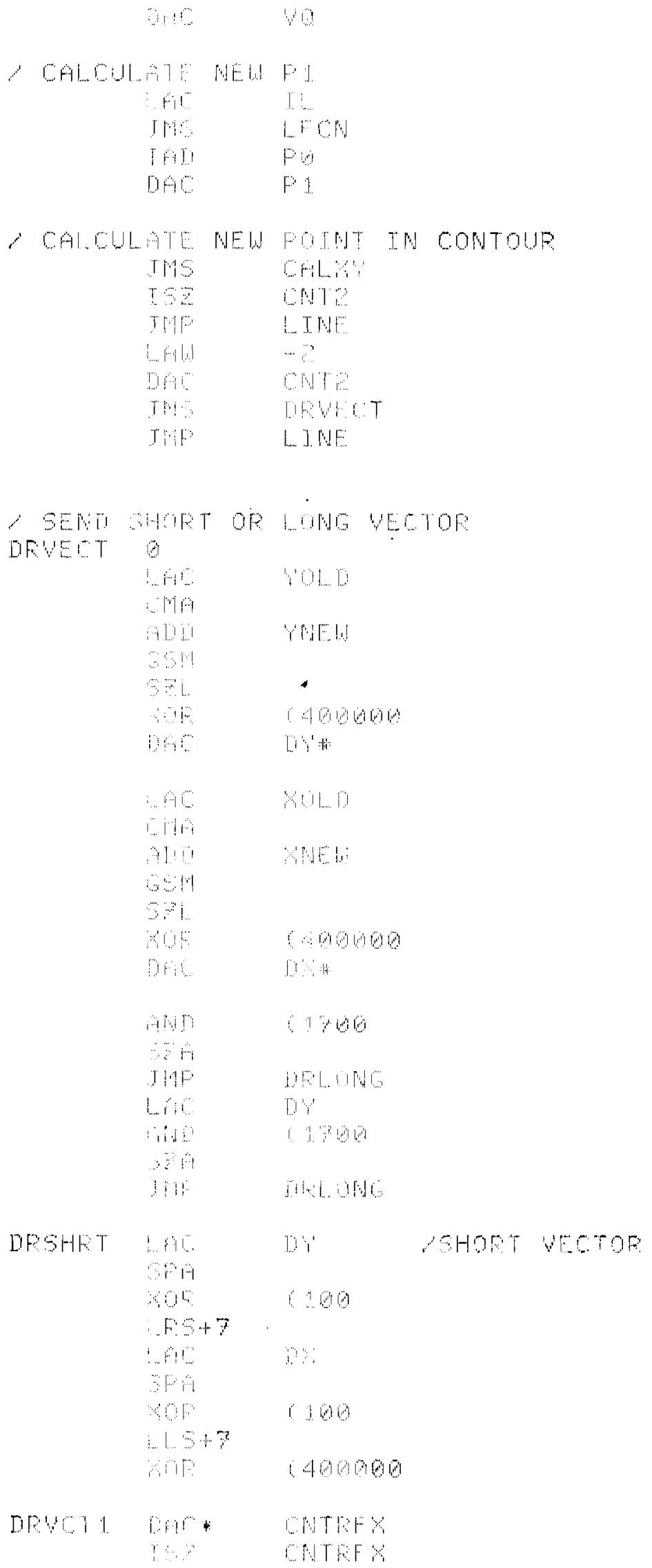




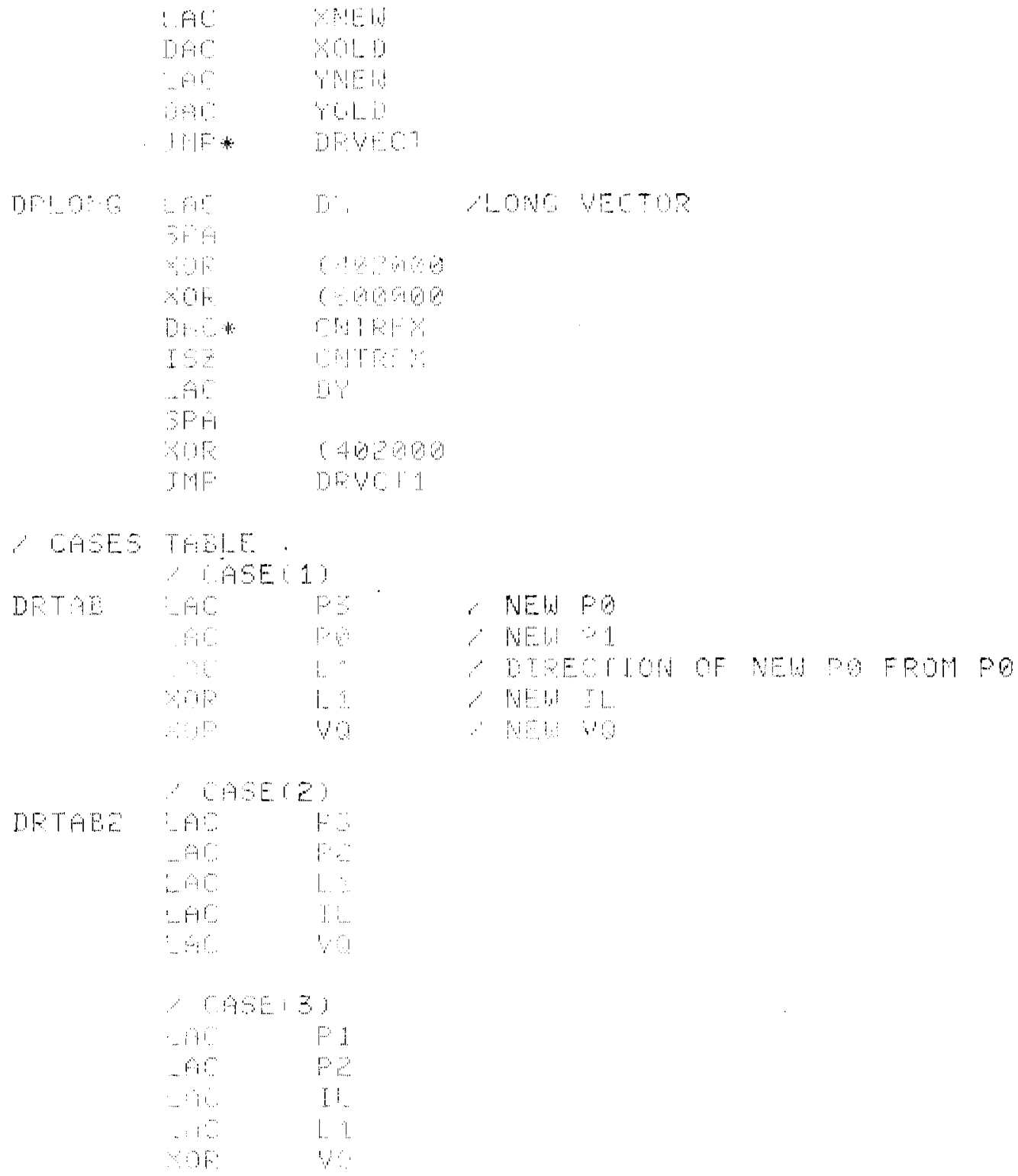




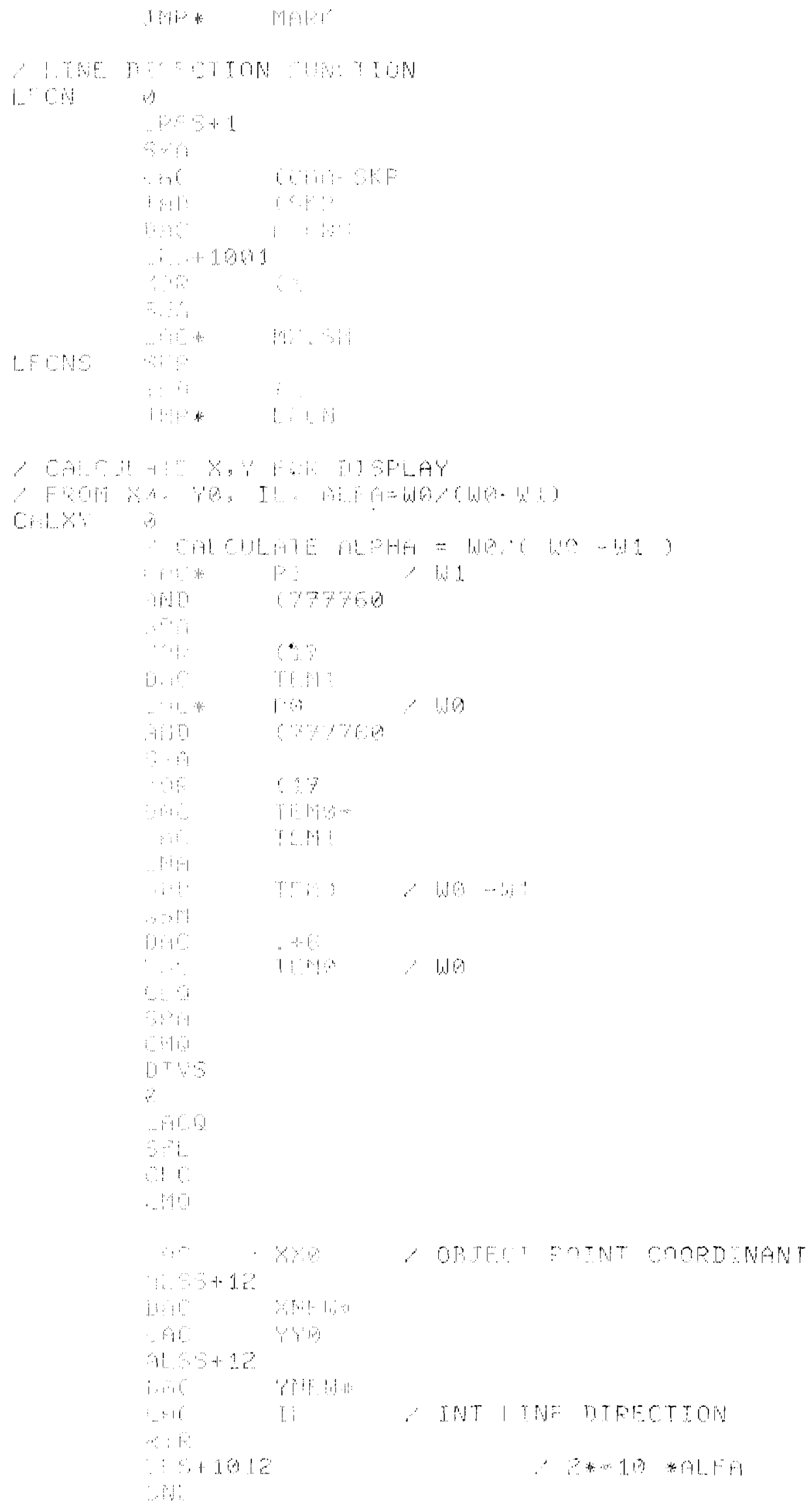




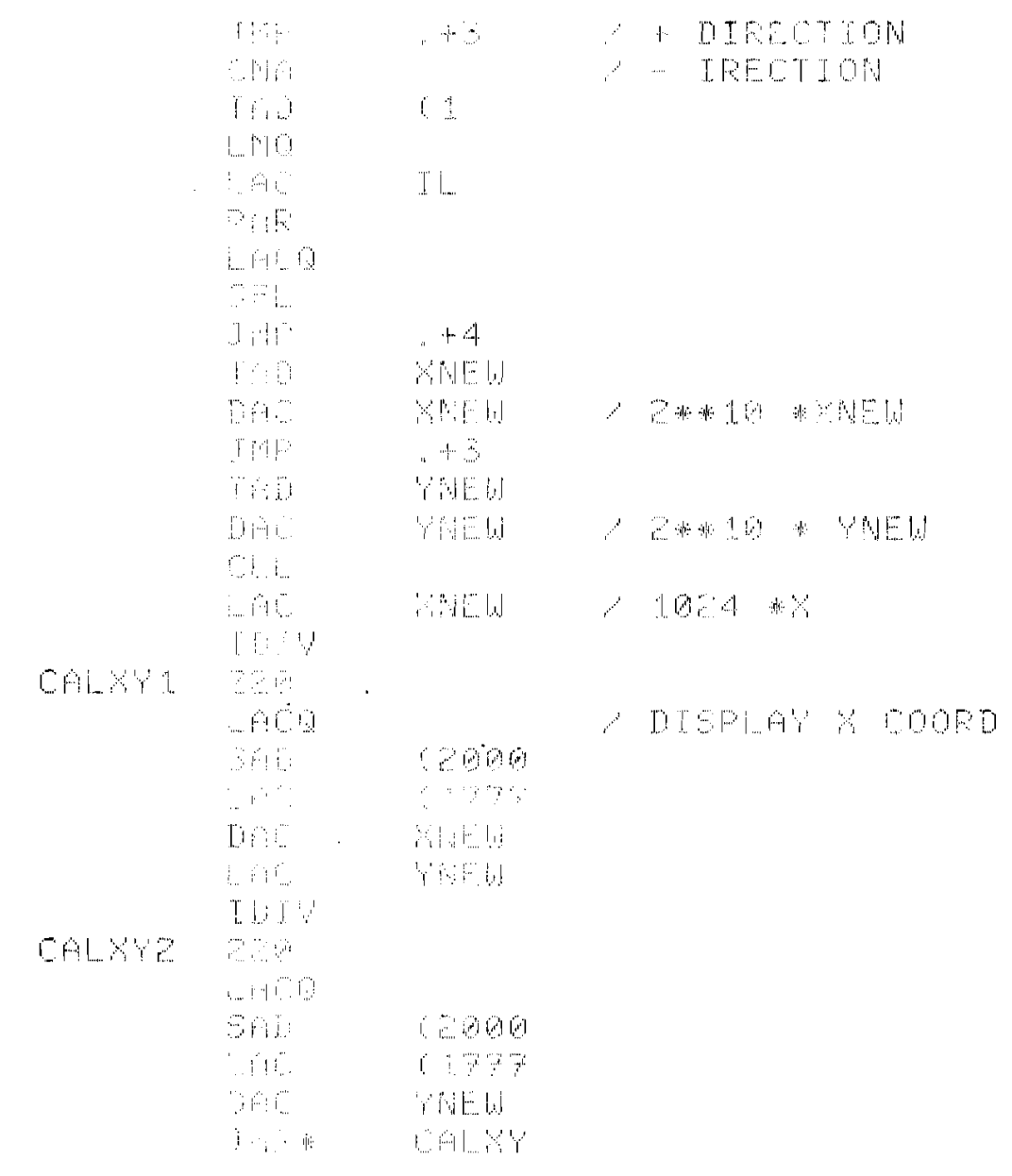

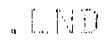


THIS 19 THE ROUTINE TO CALCULATE TRAYEL TIMES

WHS WEPSTON IS FOR USTWG TRAMSHISSTUTTY

\begin{tabular}{|c|c|c|}
\hline 1 & KFoITIOH & (I) \\
\hline & $x$ PosI IOW & $(I+1)$ \\
\hline & Y'POSI I I OAd & I \\
\hline & YPostTan & {$[+1)$} \\
\hline & $\begin{array}{l}\text { STEP LENGTH } \\
\text { TRAYL TIME }\end{array}$ & (TOTAL. \\
\hline
\end{tabular}

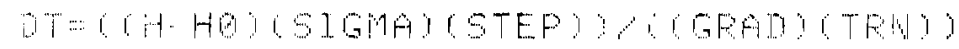

$\because$ POTEBTTEL DET

MO AOUIFIER EEET

STGHA POROSTTY

STEF STEP LEMGTH

UPITLESS

GRAD GEADIENT

MODE

TRH TFAOMISEITY

FEETMONOE

$F T-2$ DGY

\section{DIMIESTOW IDI I , DATACZB)}

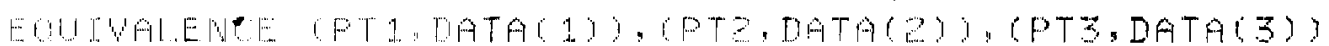

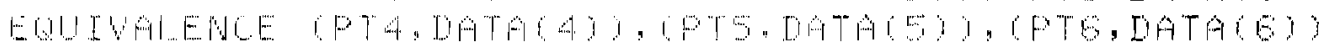

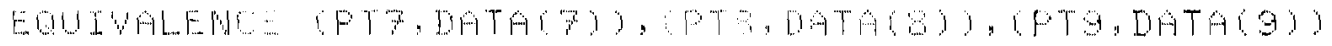

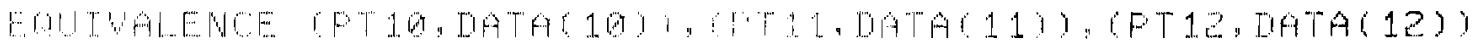

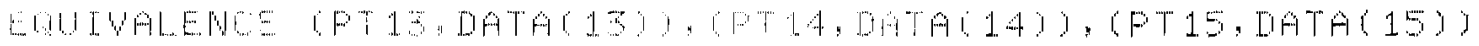

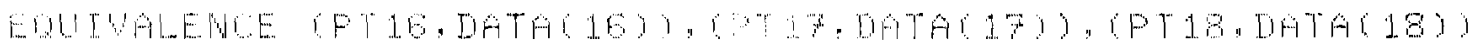

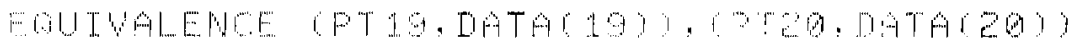

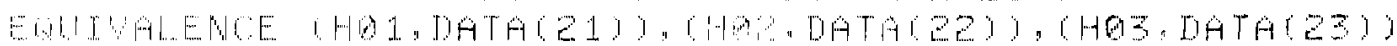

COUIUALEWE (HOA DATACZA)

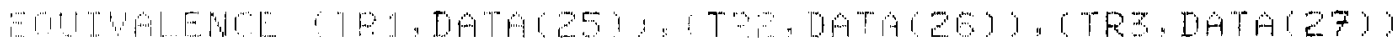

EOUYALENCE TR4, WATAC28)

$E$

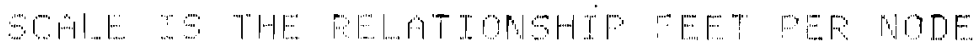

DATA SCALE LOOQ.

G

DATA STGM 20 , 1 .

C.

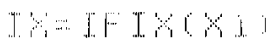

$2:=1 F+1919$

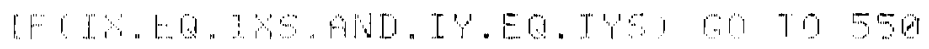

$195=I \%$

IYS - J

CALL GRDFH I I , IY:ID

$04500 \cdot T=1,24,1$

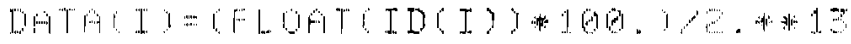

500 WONTINUE

$190325 \quad I-25,28,1$

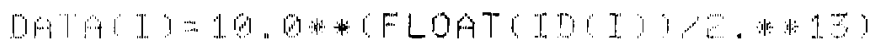

525 CONIHUE

C.

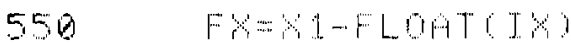

$F Y=Y-F L O A T I Y$ C

E 
C

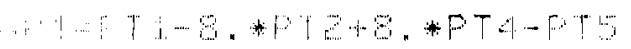

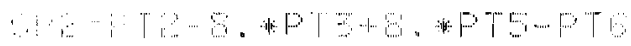

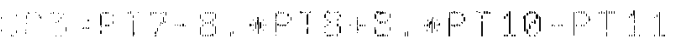

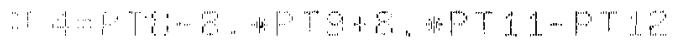

$61=69+F 8+692-691)$

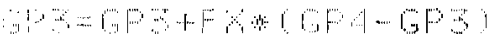

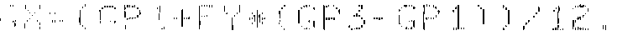

C.

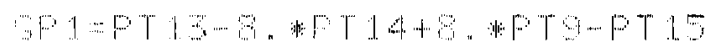

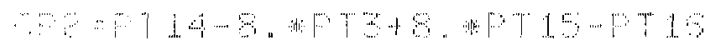

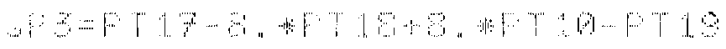

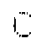

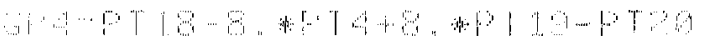

C

G $10641+40602-651)$

$6 P 2=6 P 3+F+19 P 4-6 P 3 ?$

E

G.

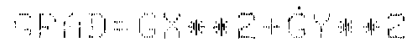

¿

GMJ $=5$ TET GRAD)

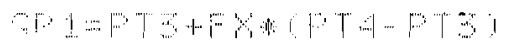

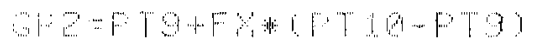

wat

$c$

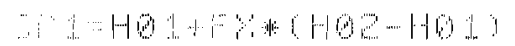

a P HOS F H

$\mathrm{C}$

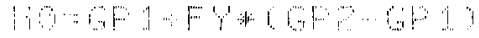

C

$C P 1=\mid P 1+F P(T P Z-1 R 1)$

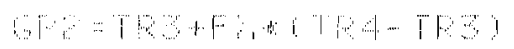

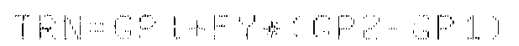

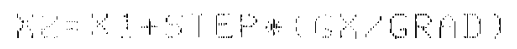

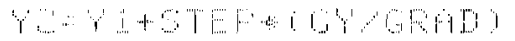

$\mathrm{C}$

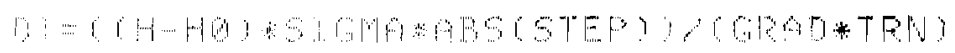

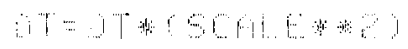

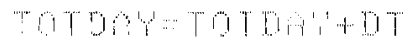

G

PETUPH

a.t. 
APPENDIX D

TRANSMISSIVITY PROGRAM (STEADY STATE) IISTING 


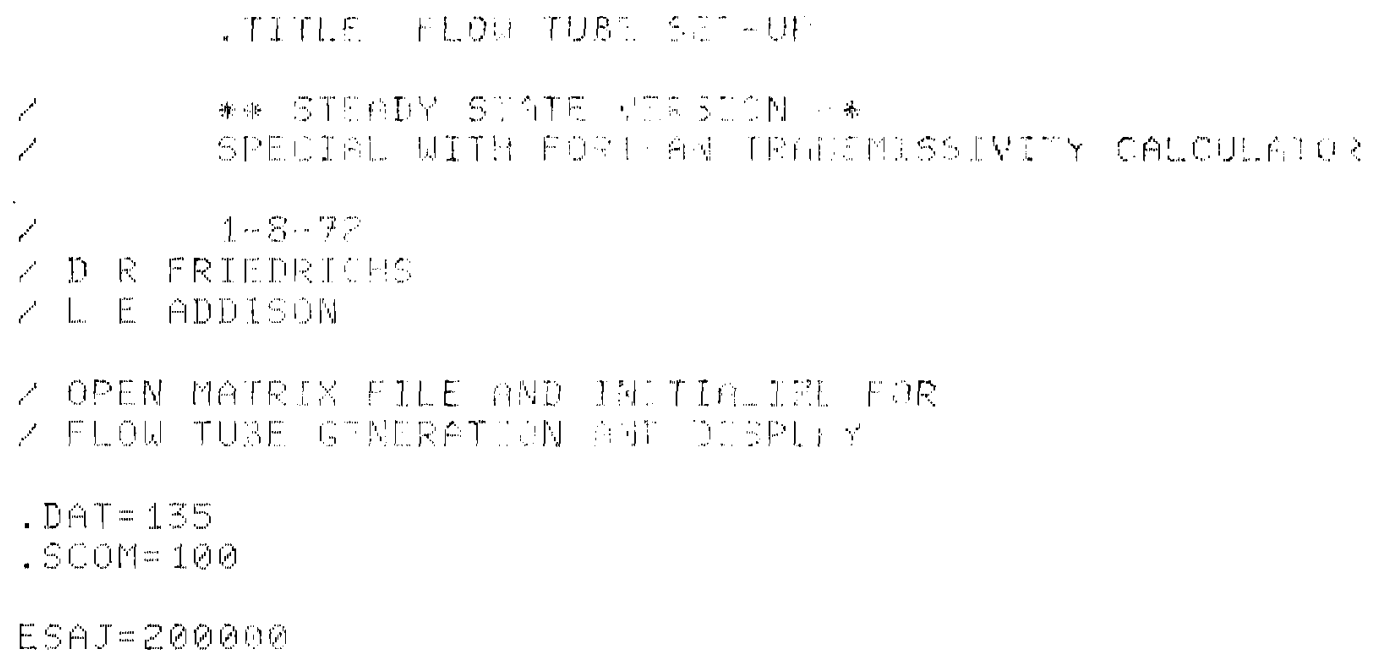




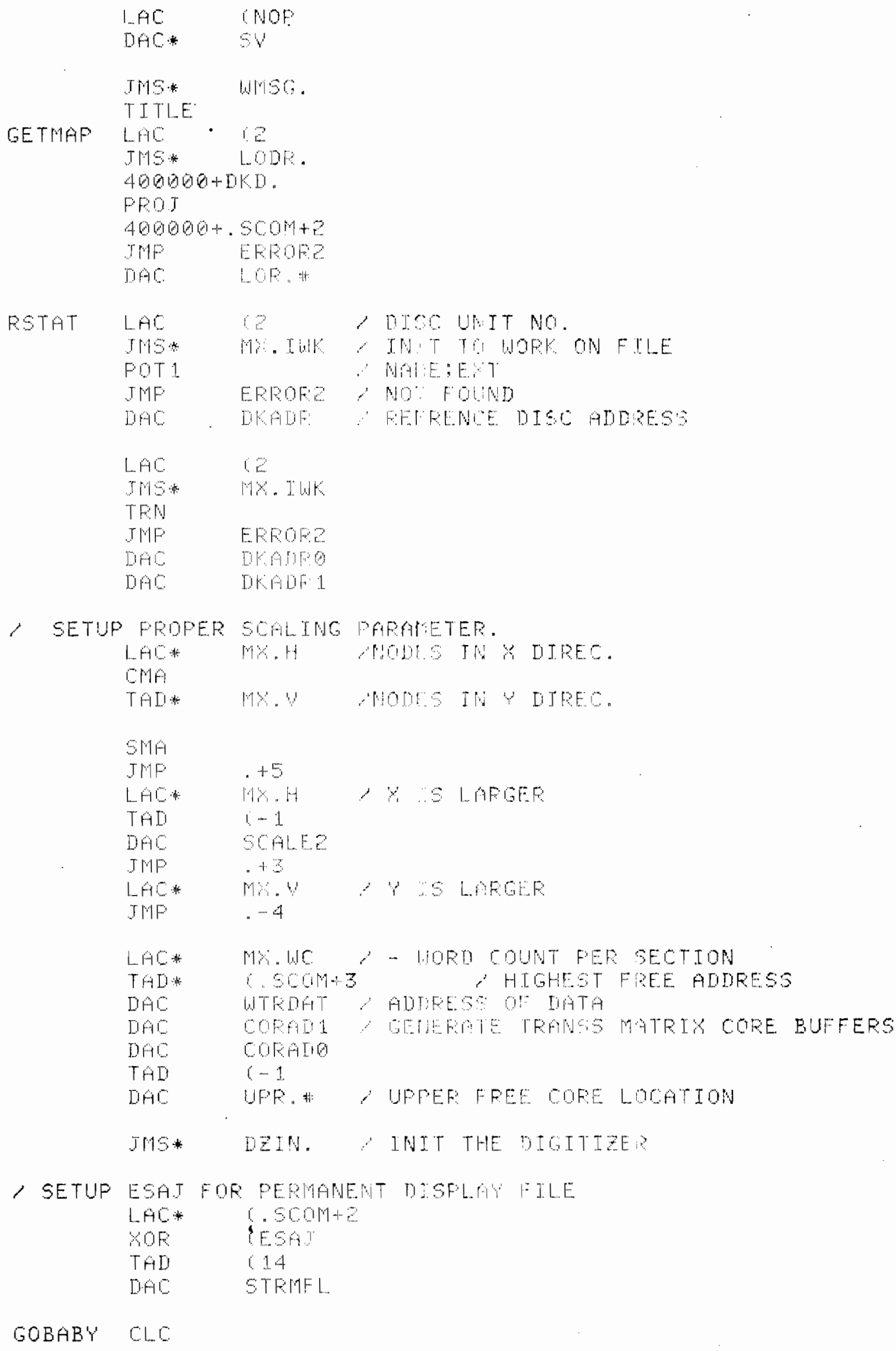




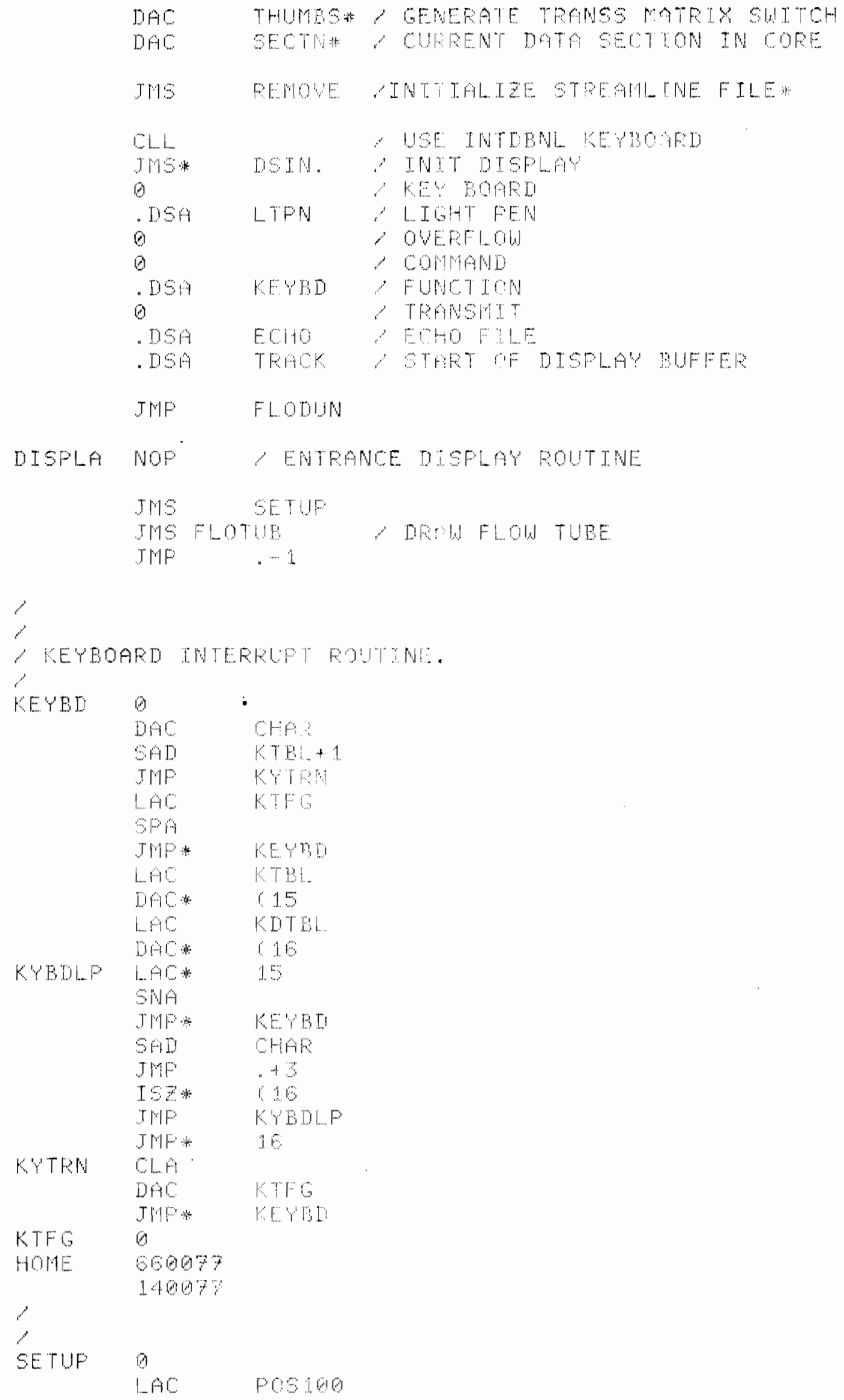




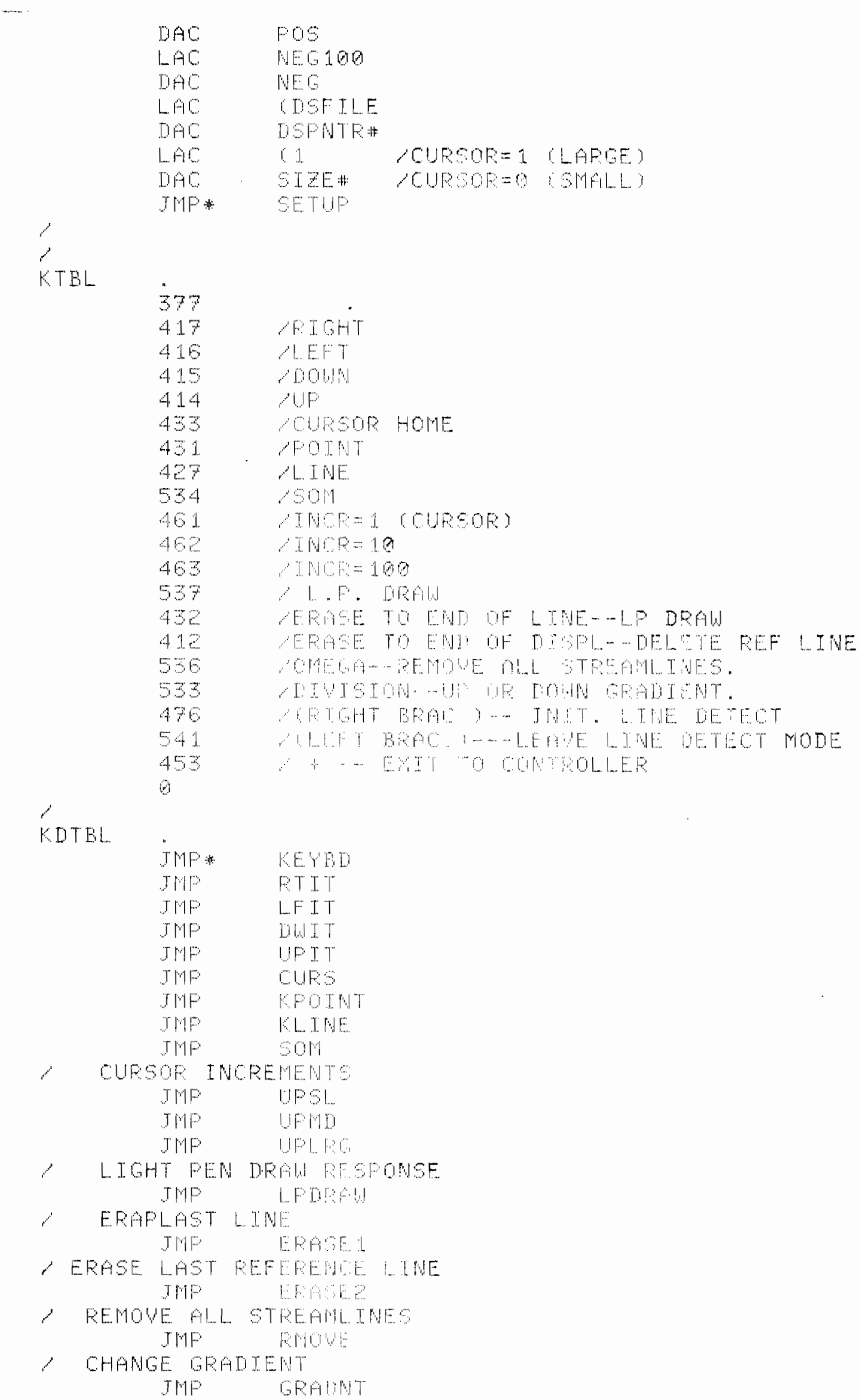




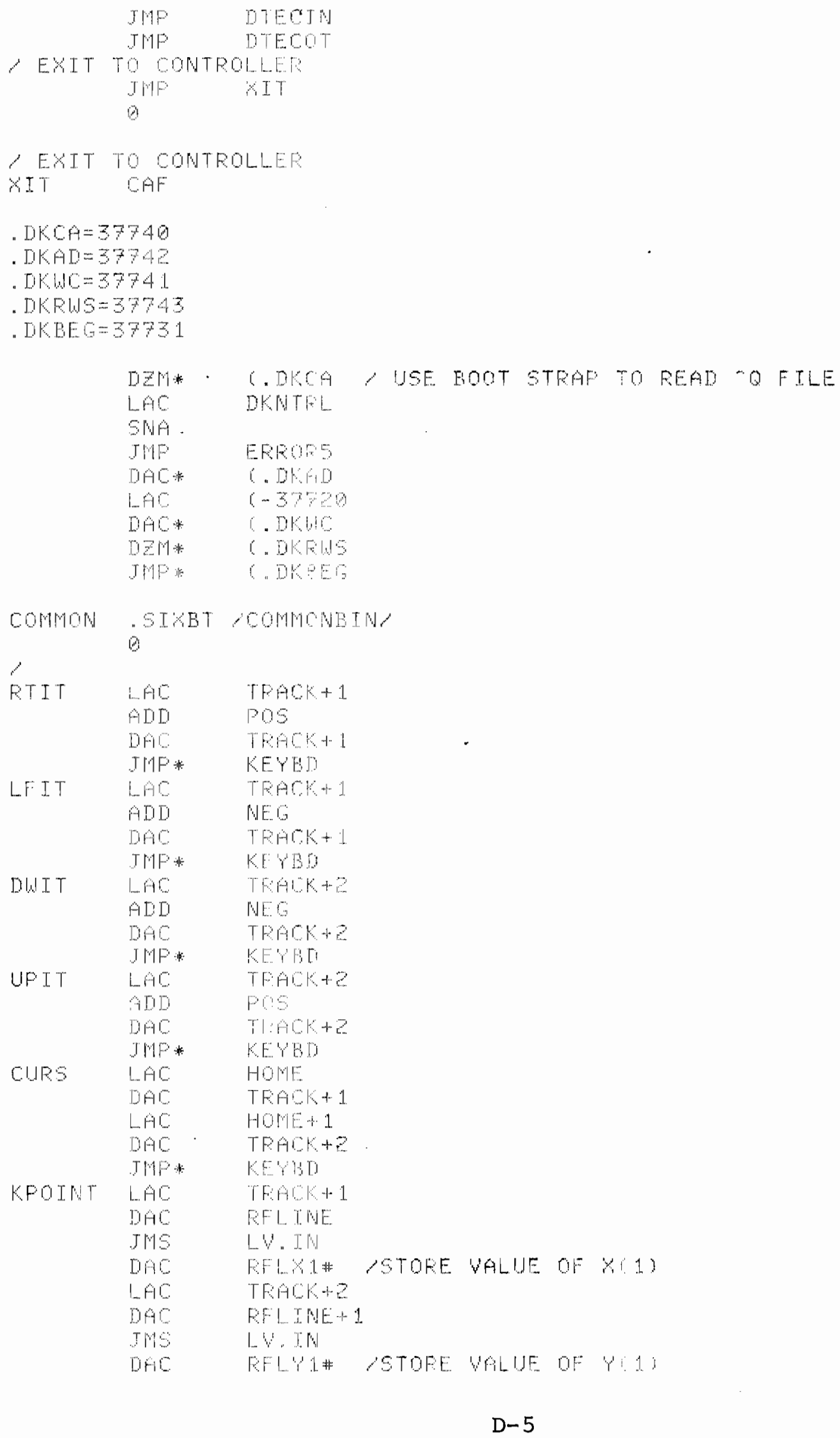




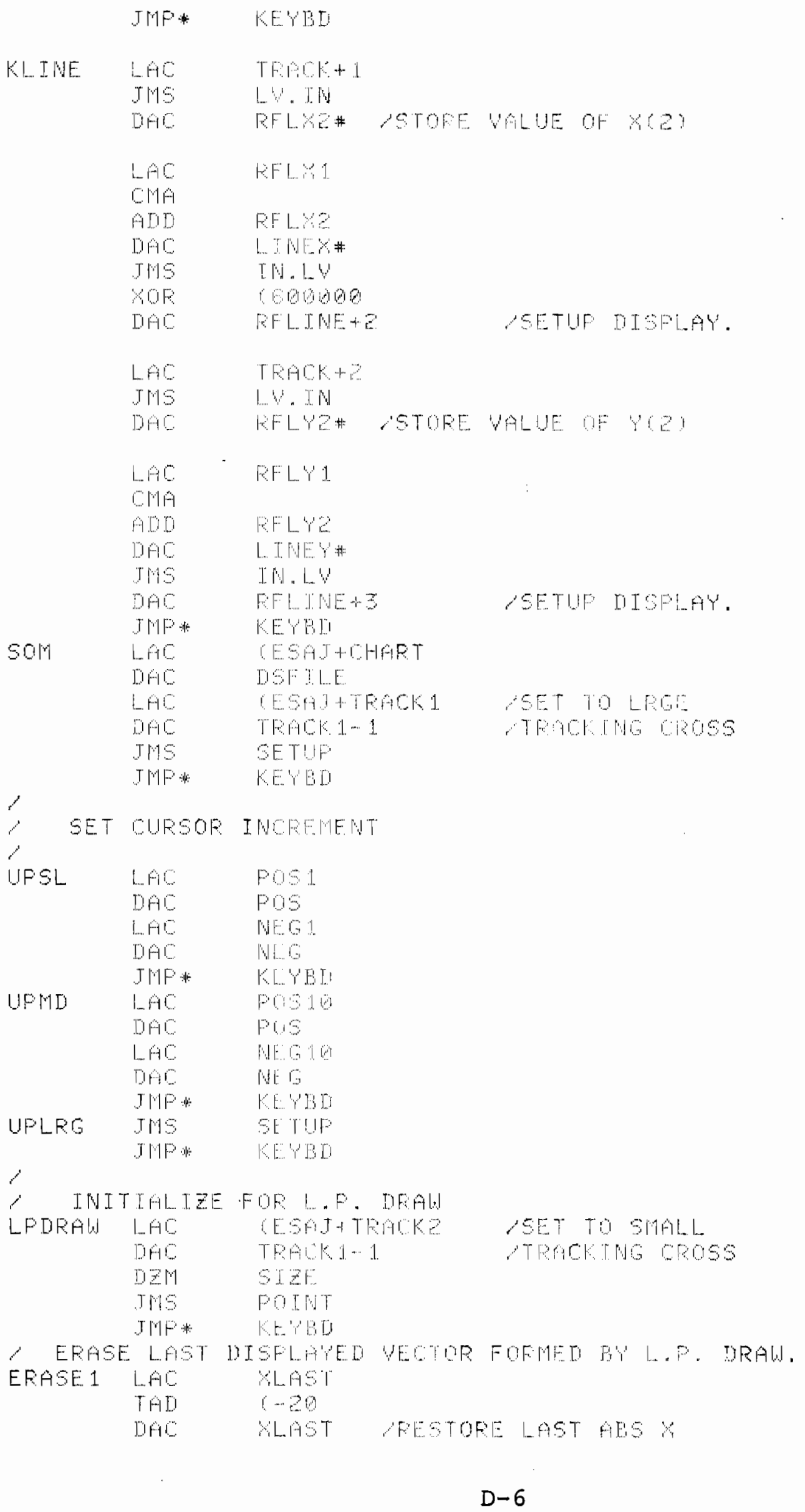




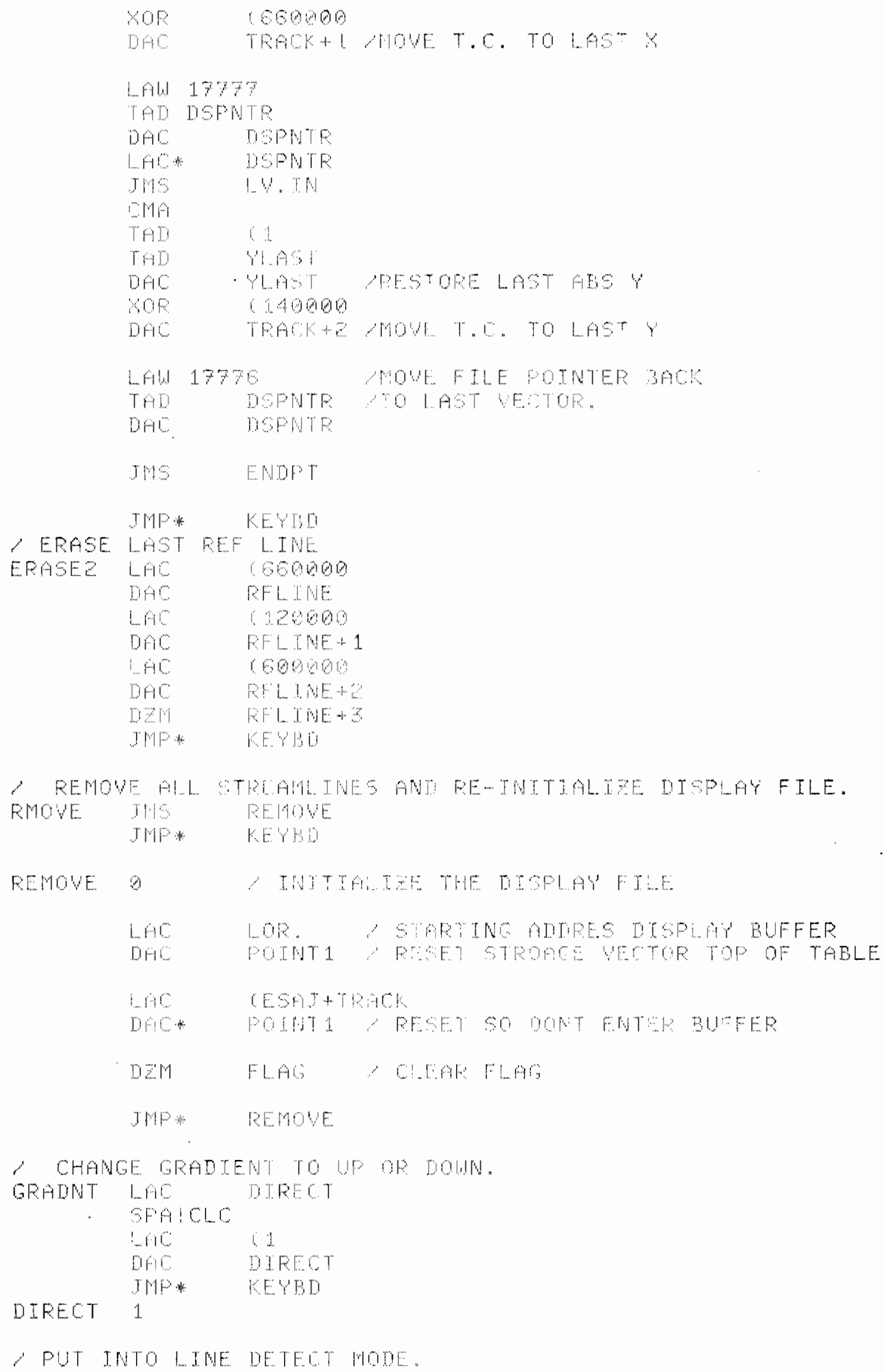




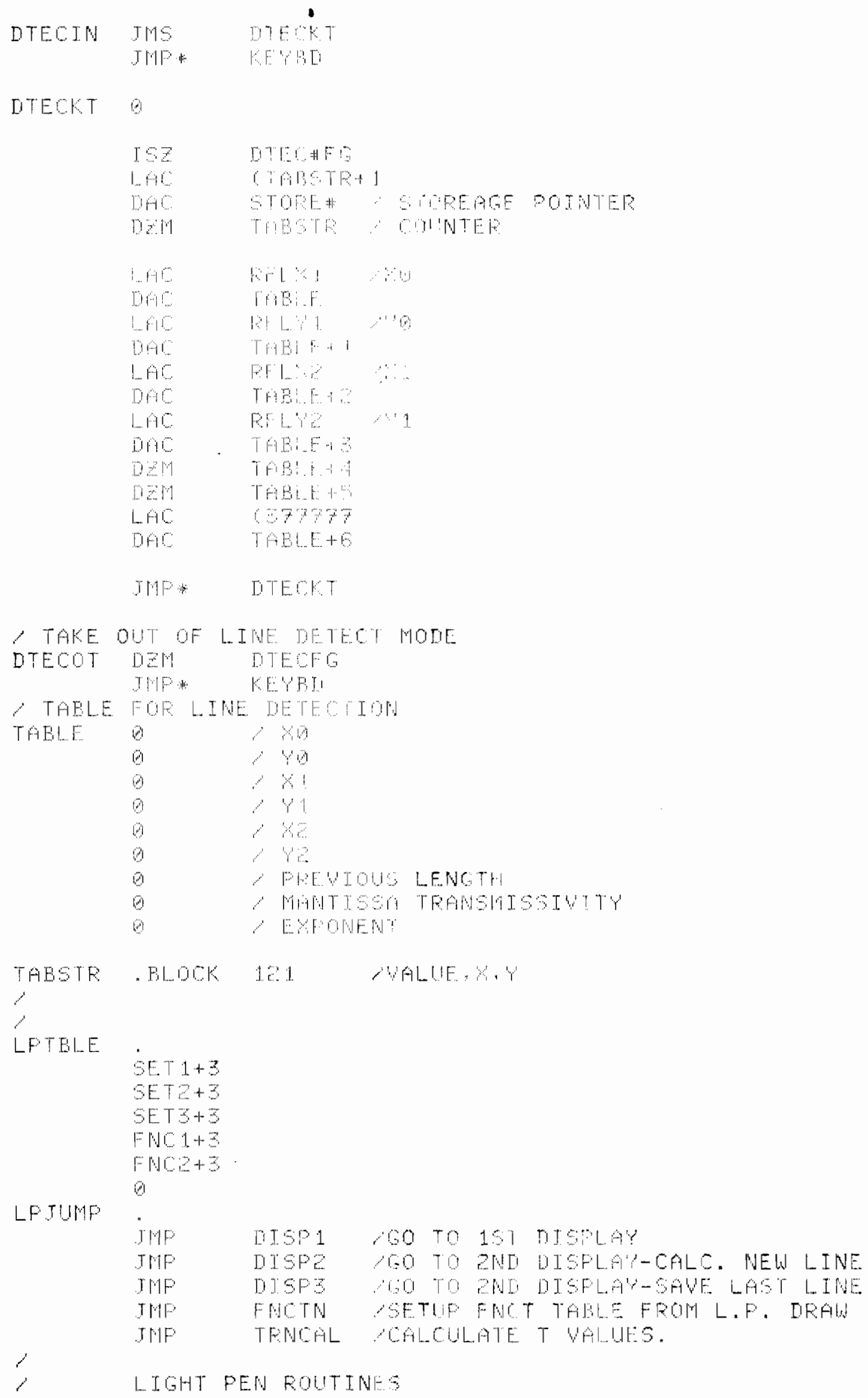




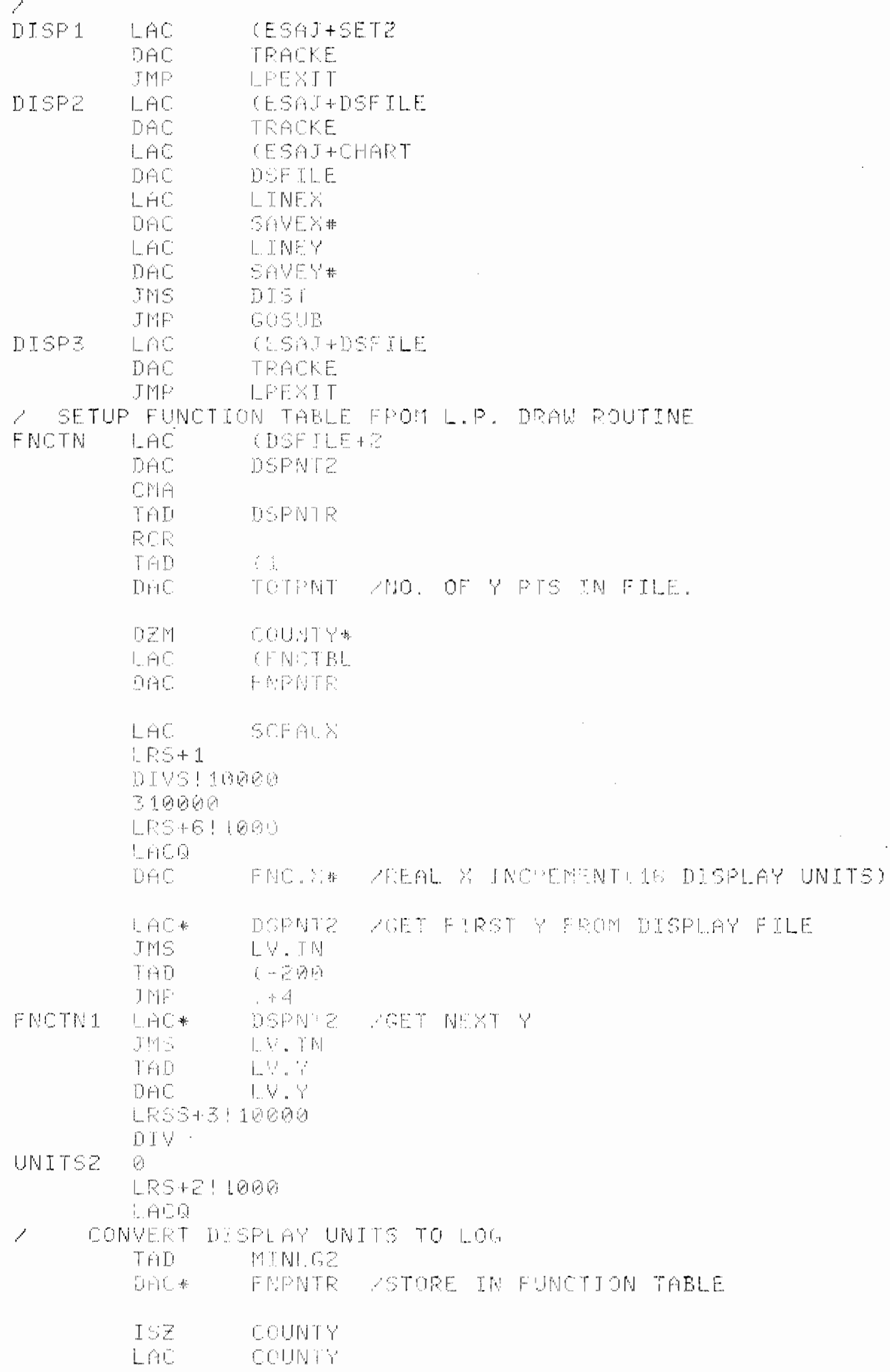




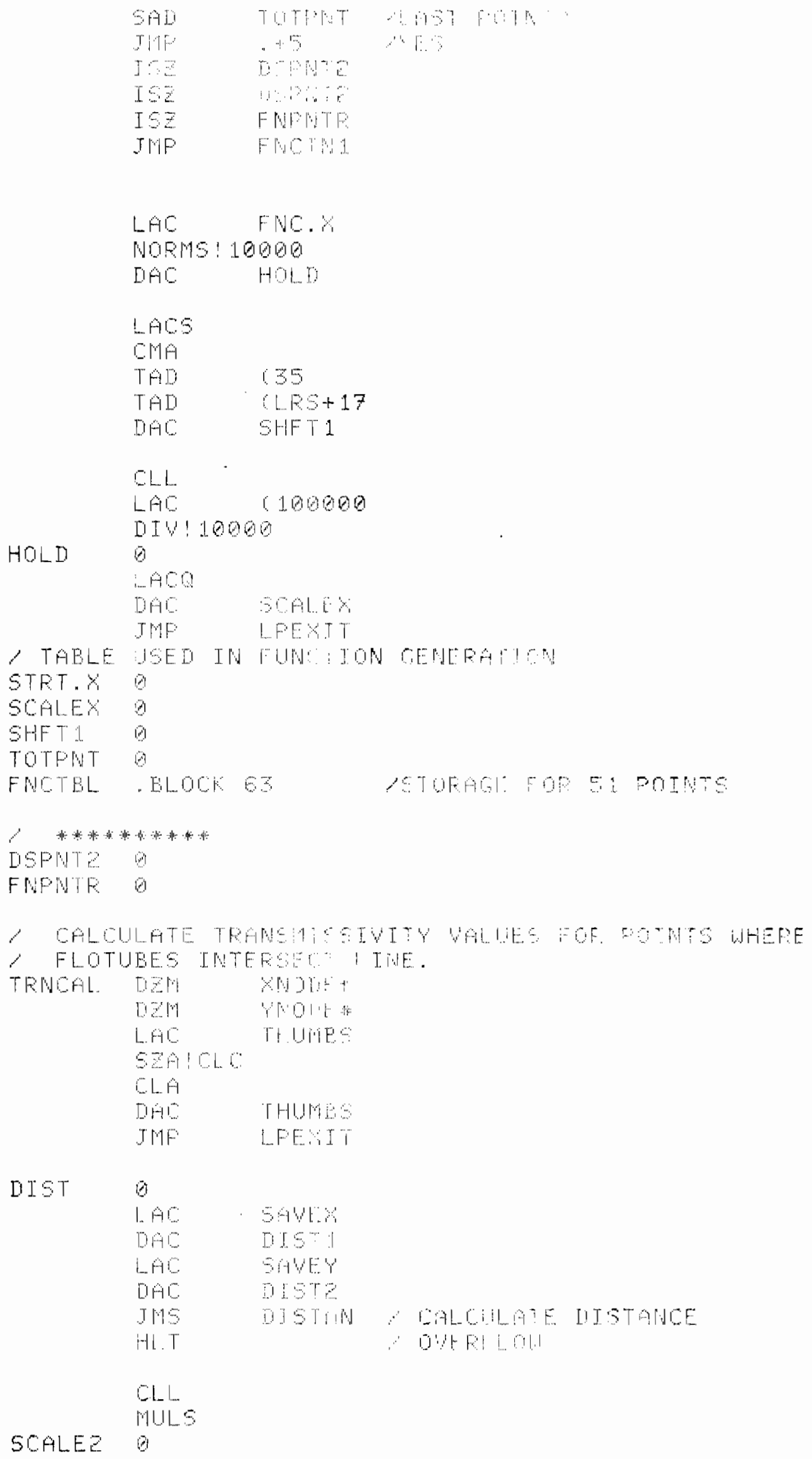




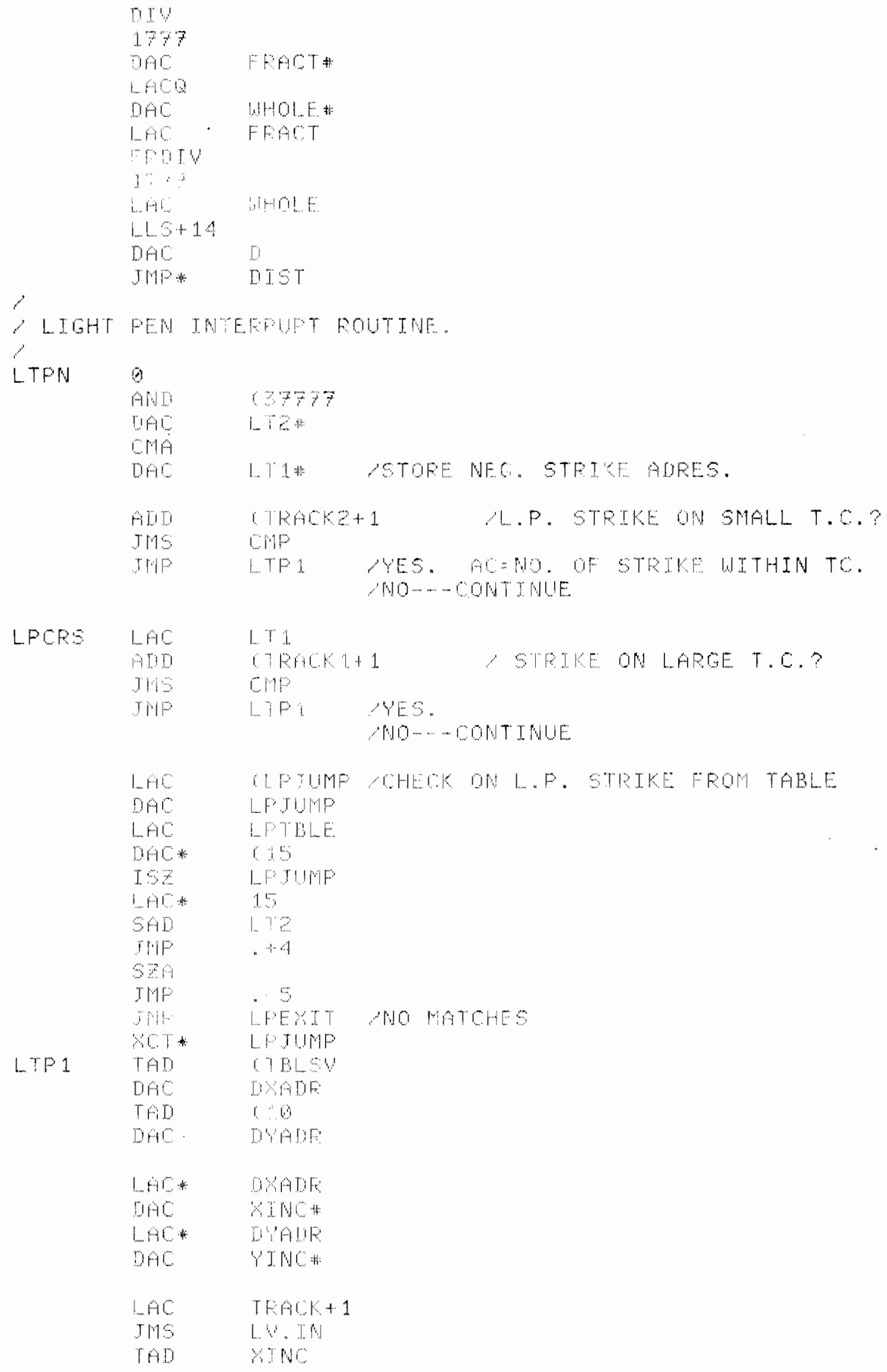




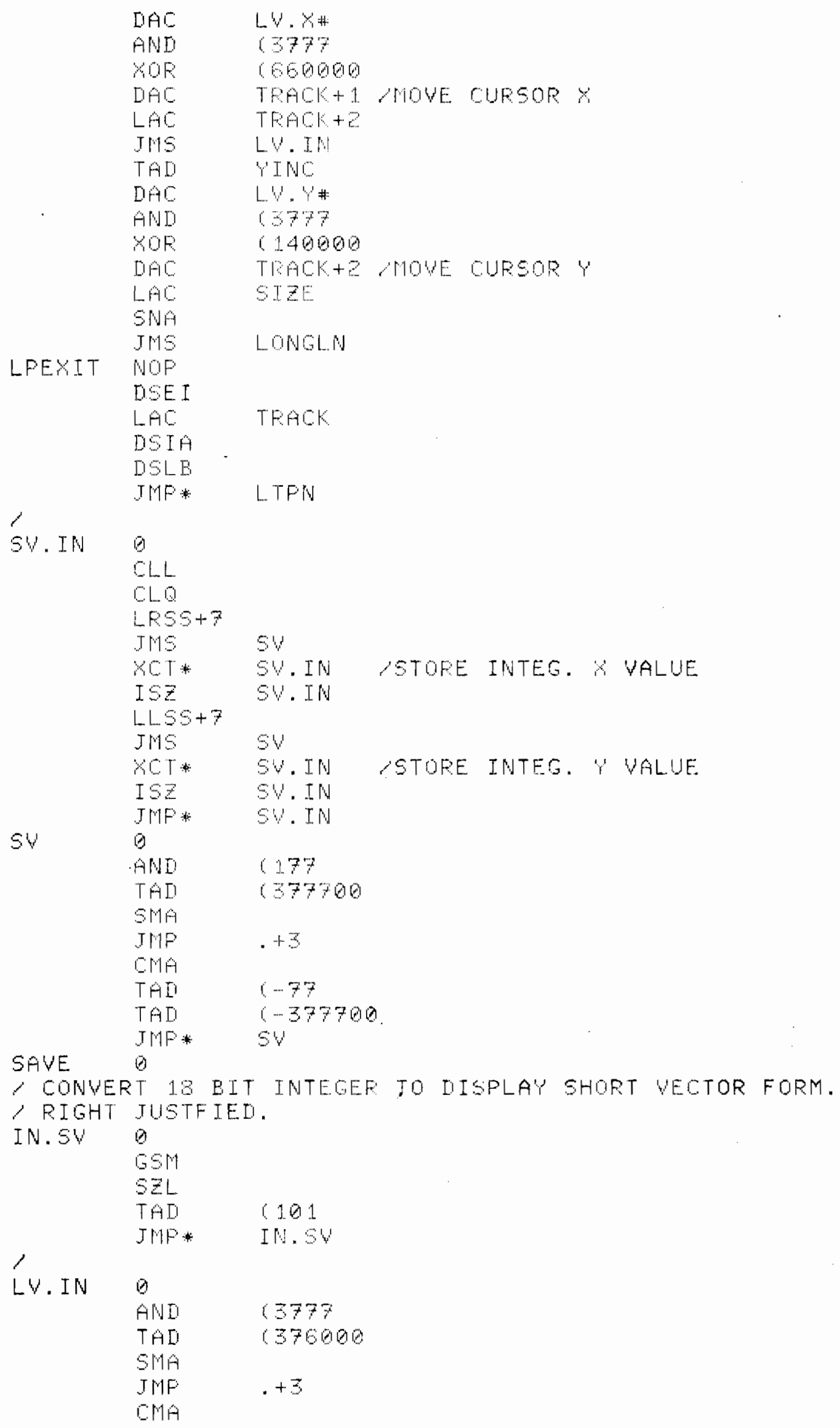




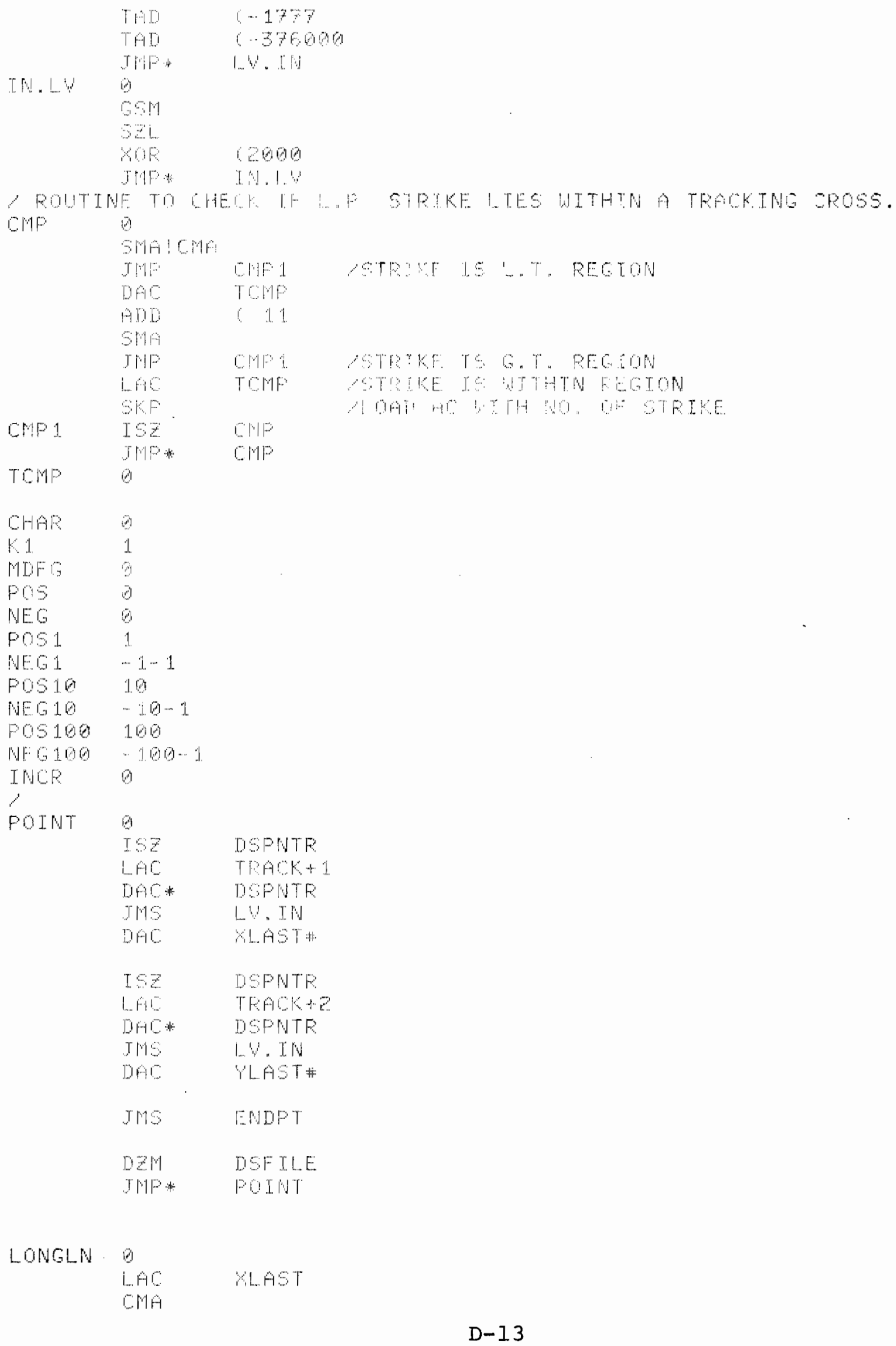




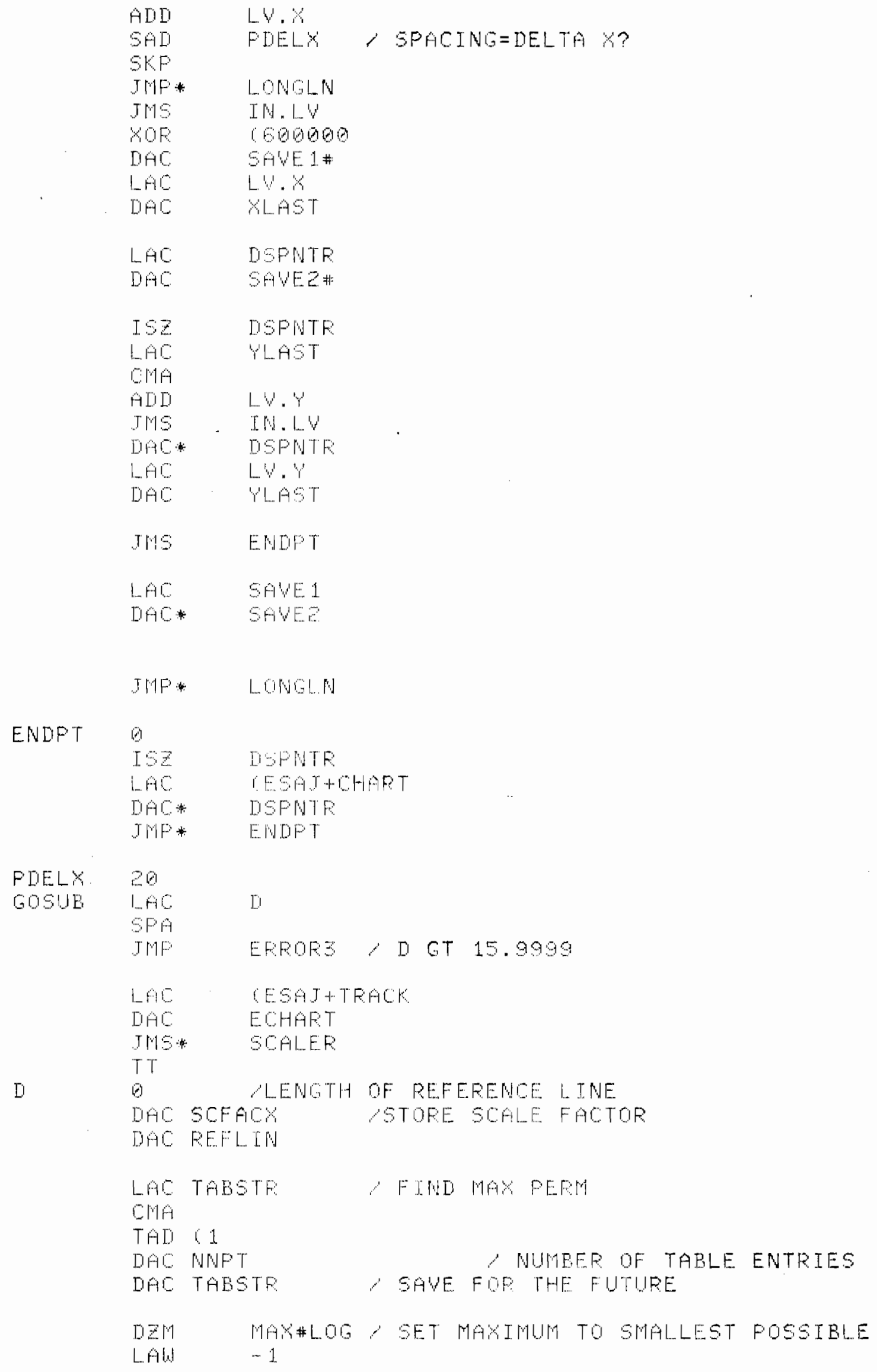




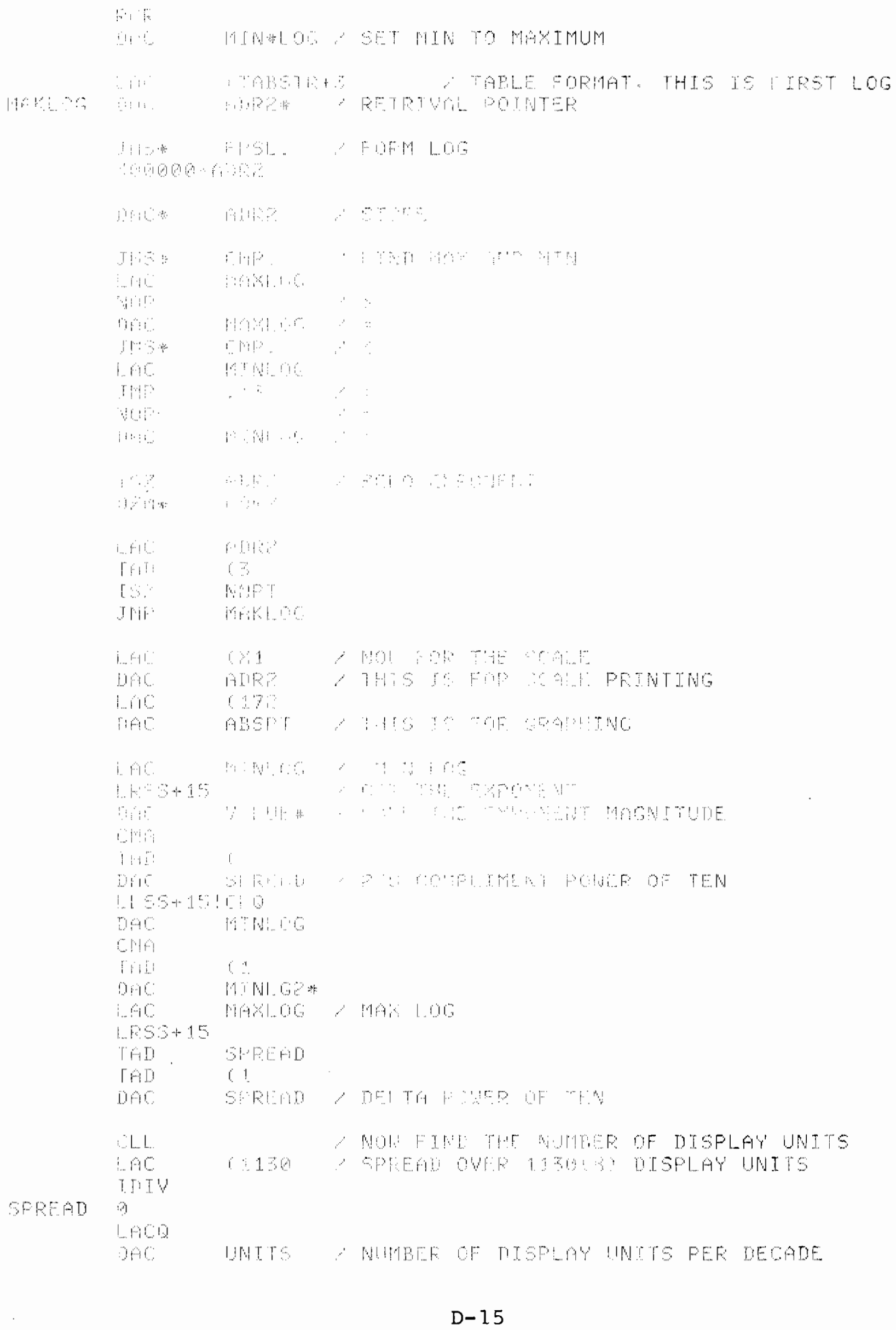




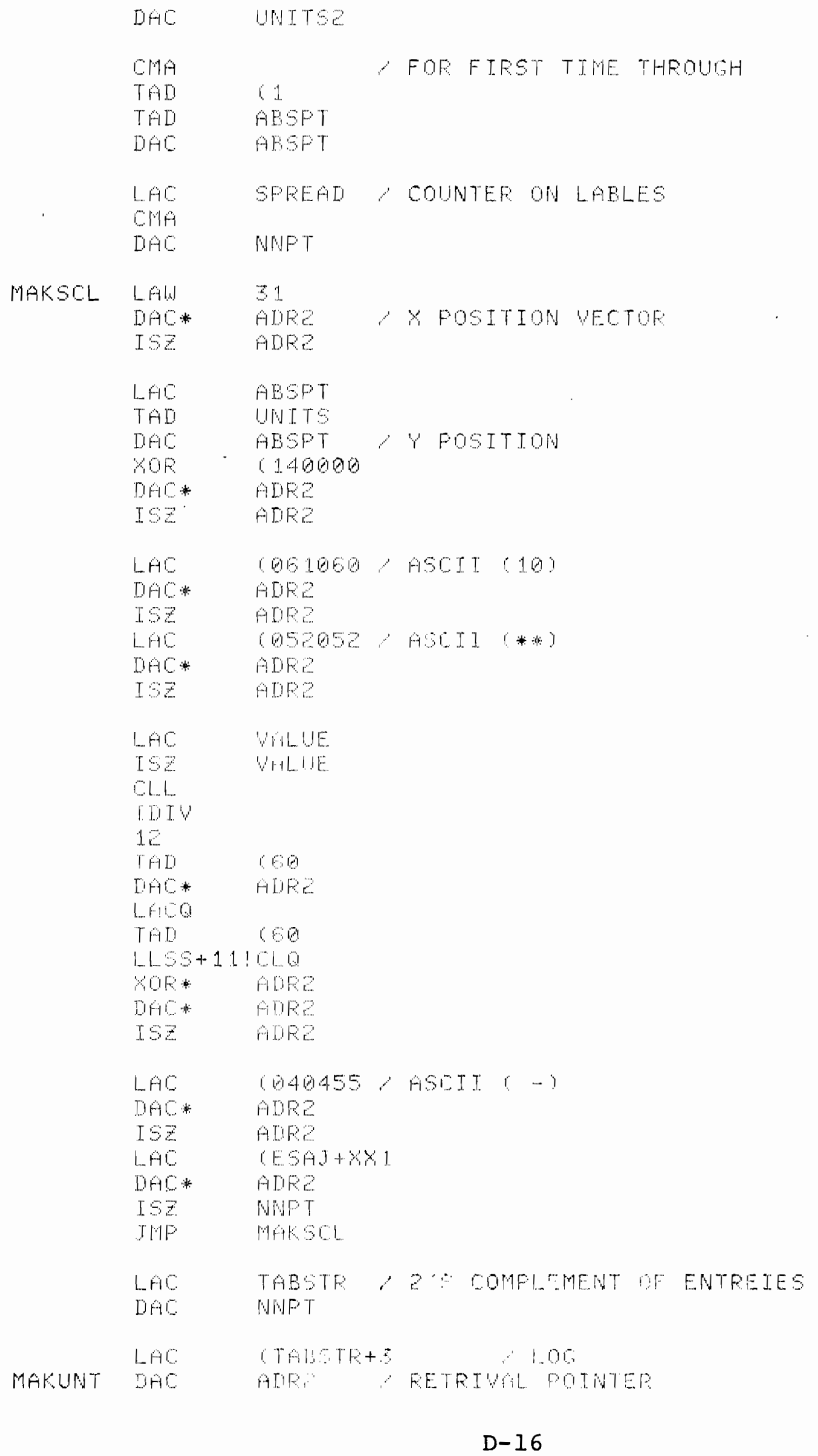




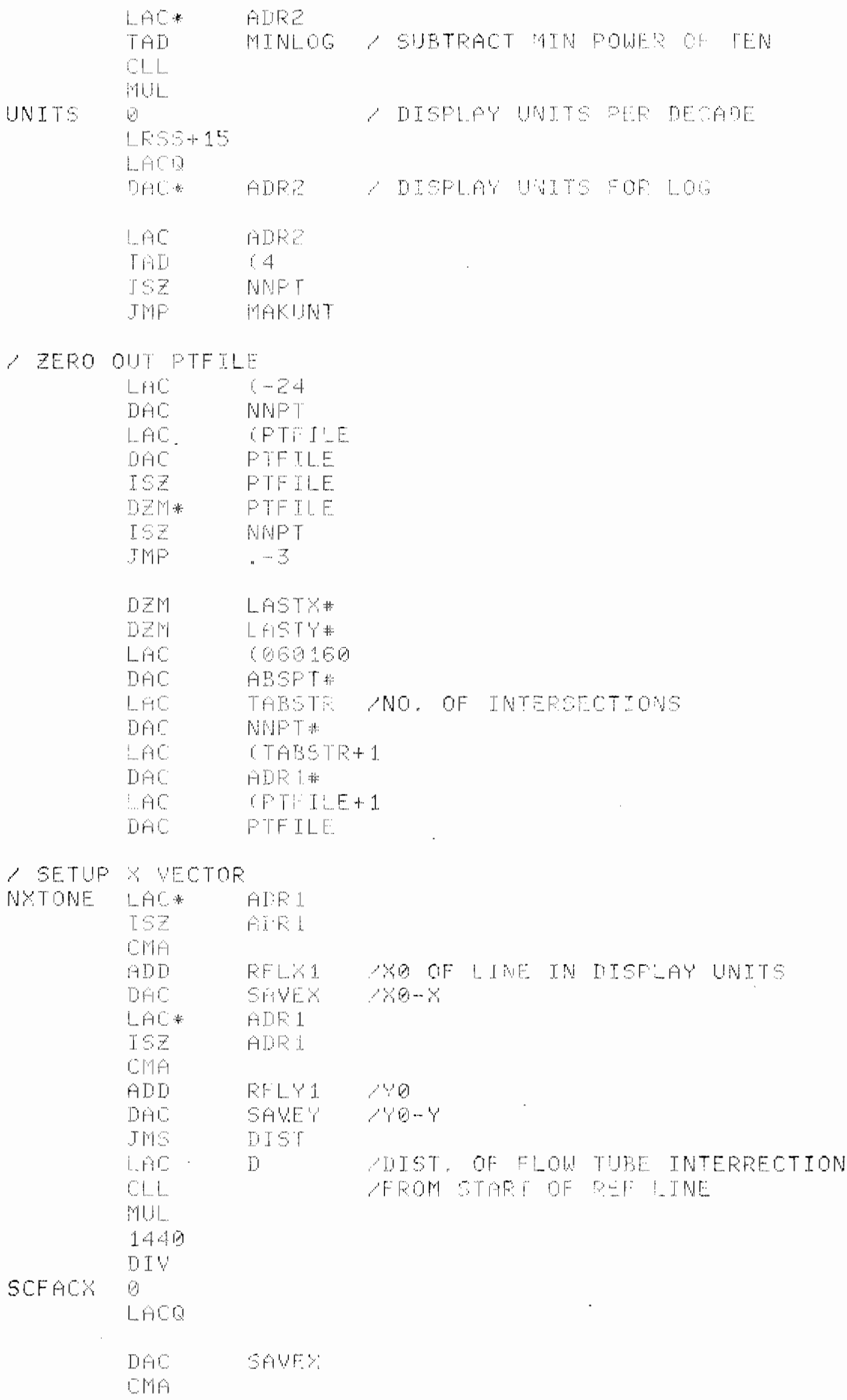




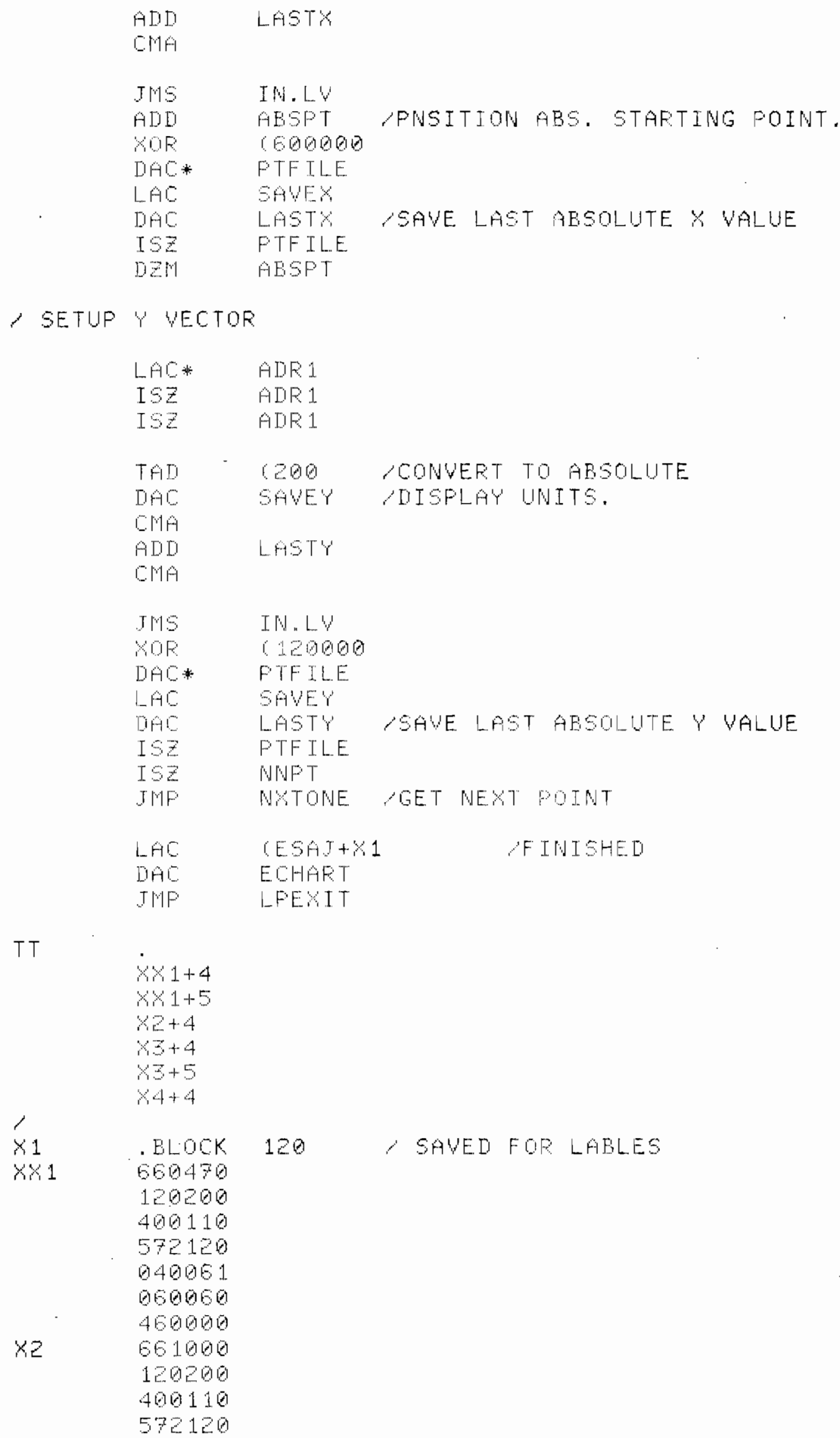




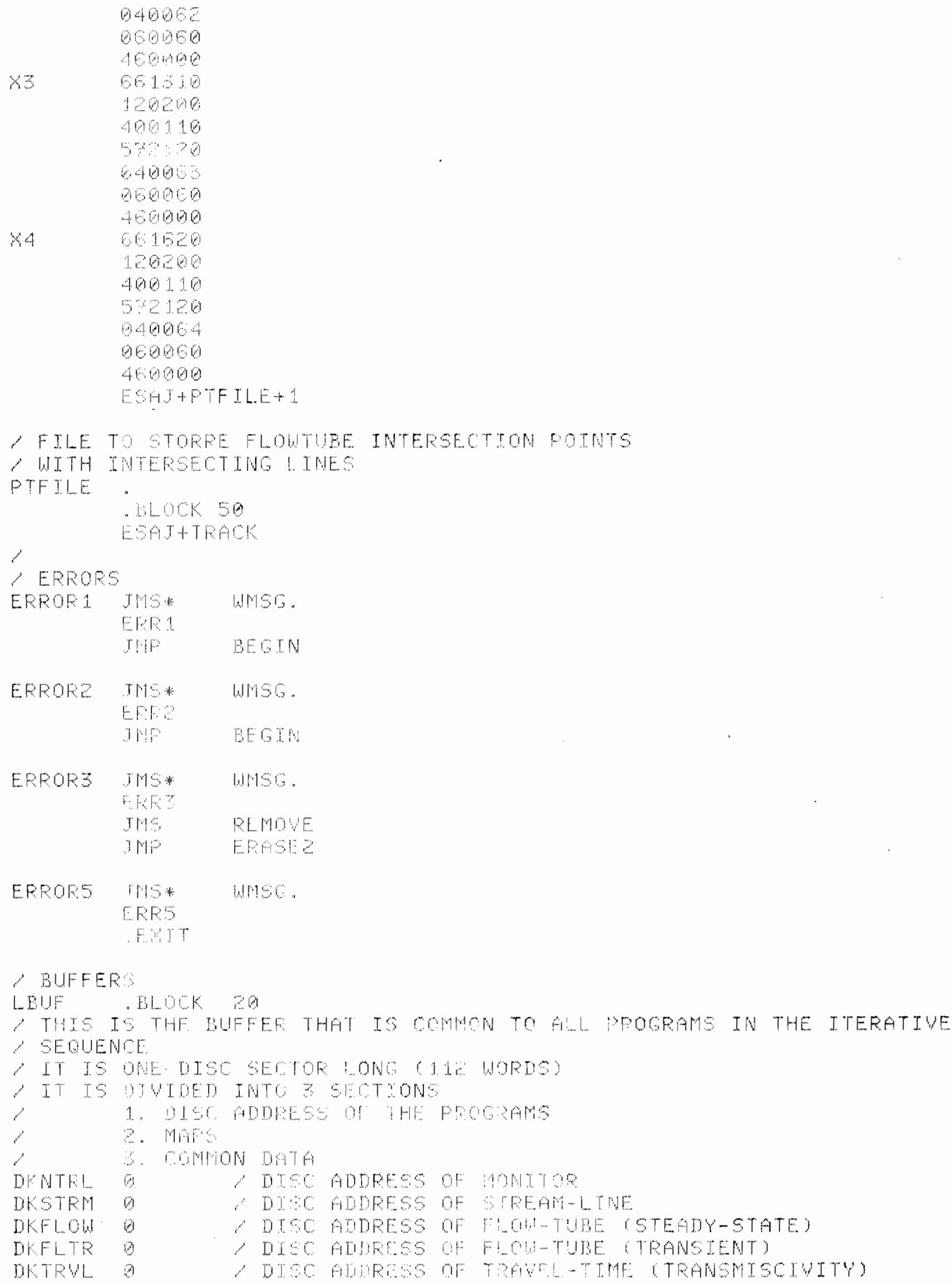




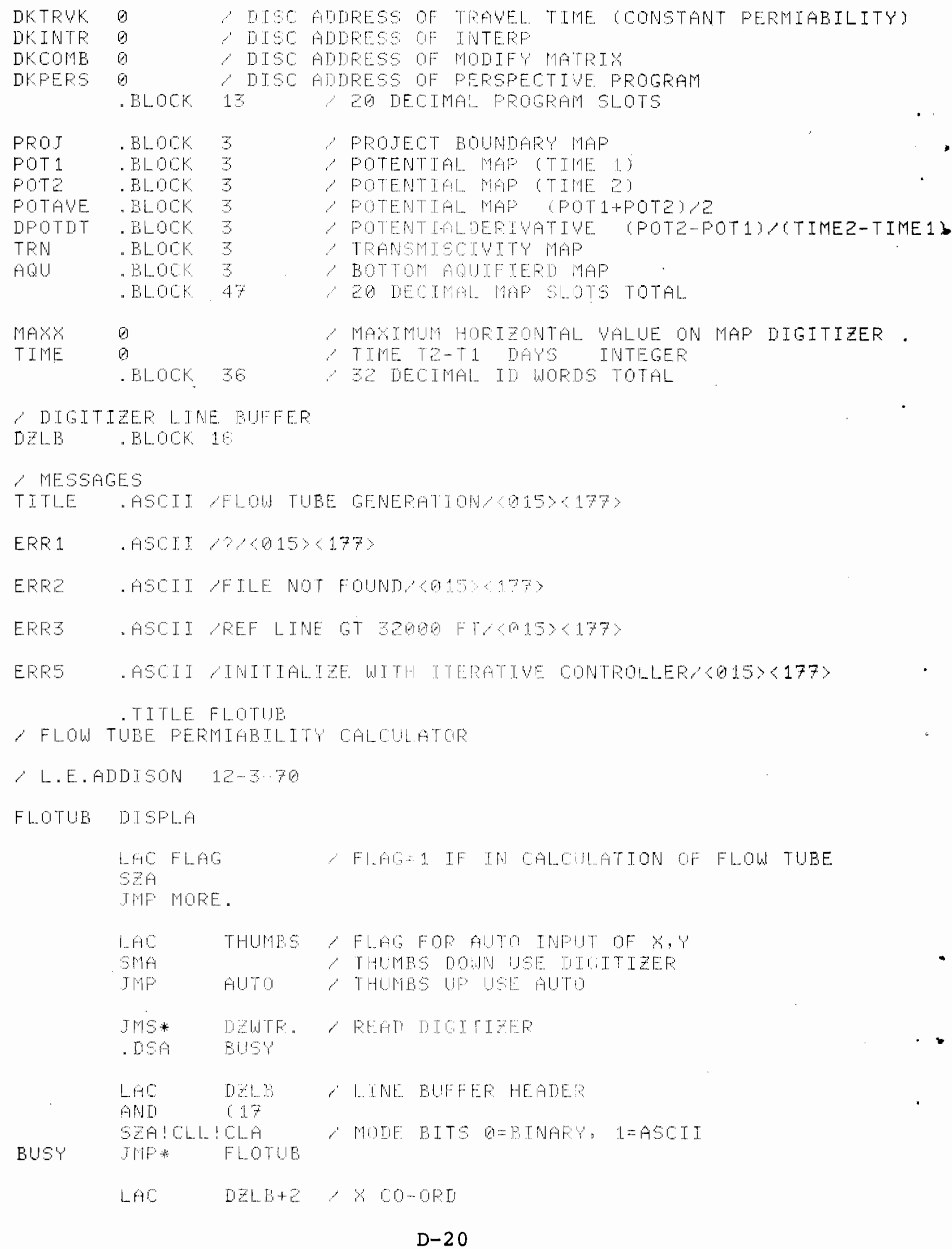




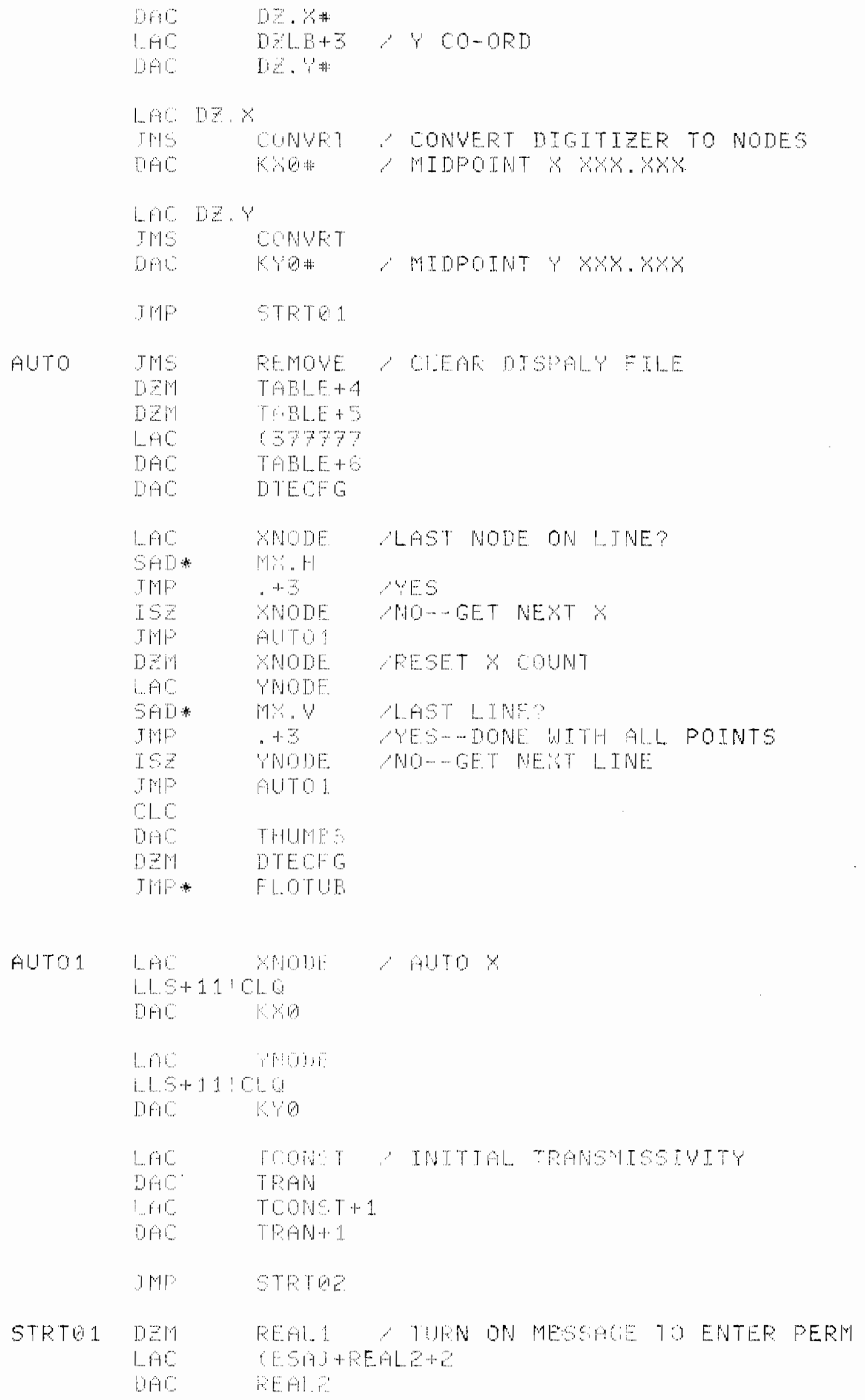




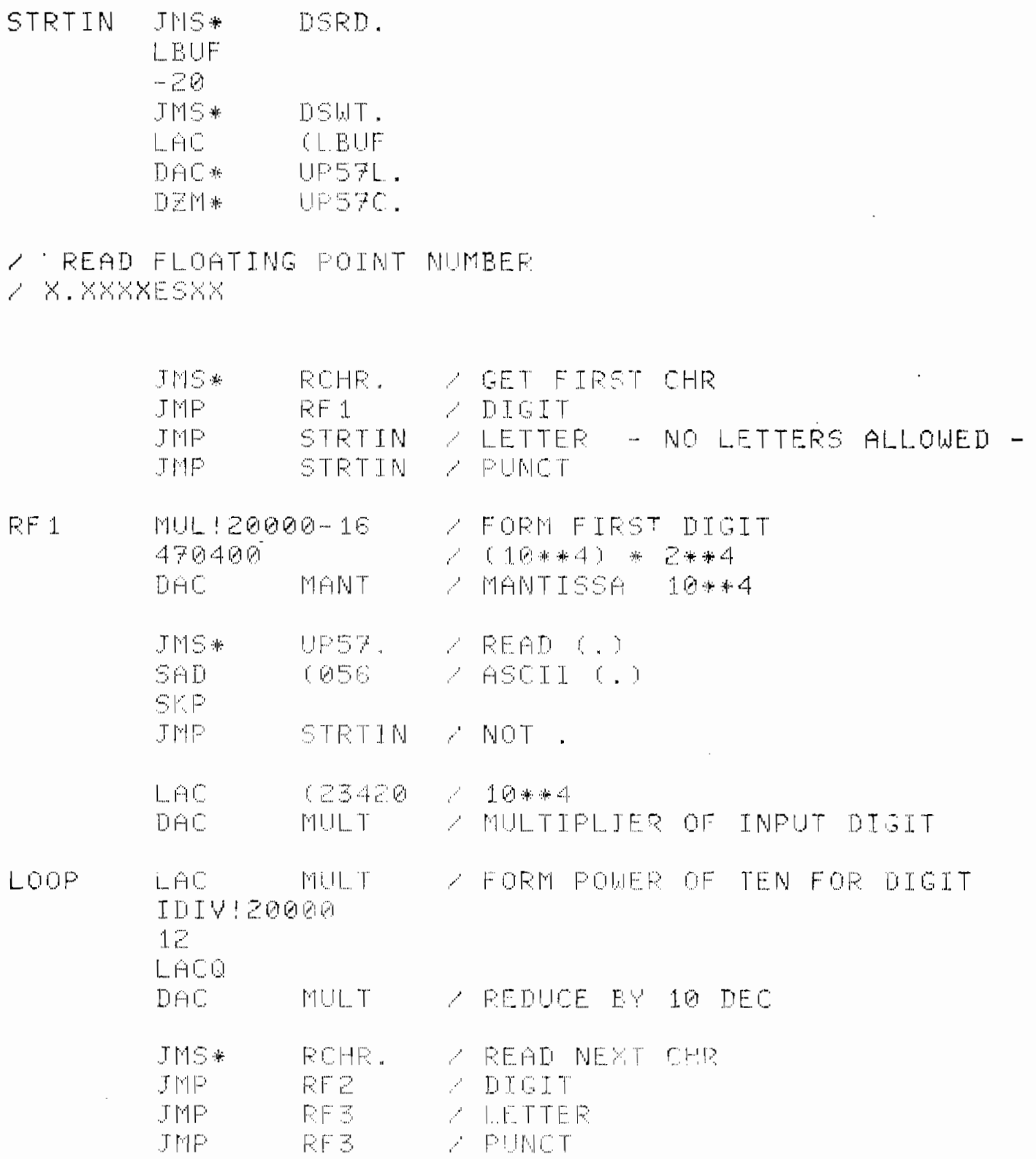




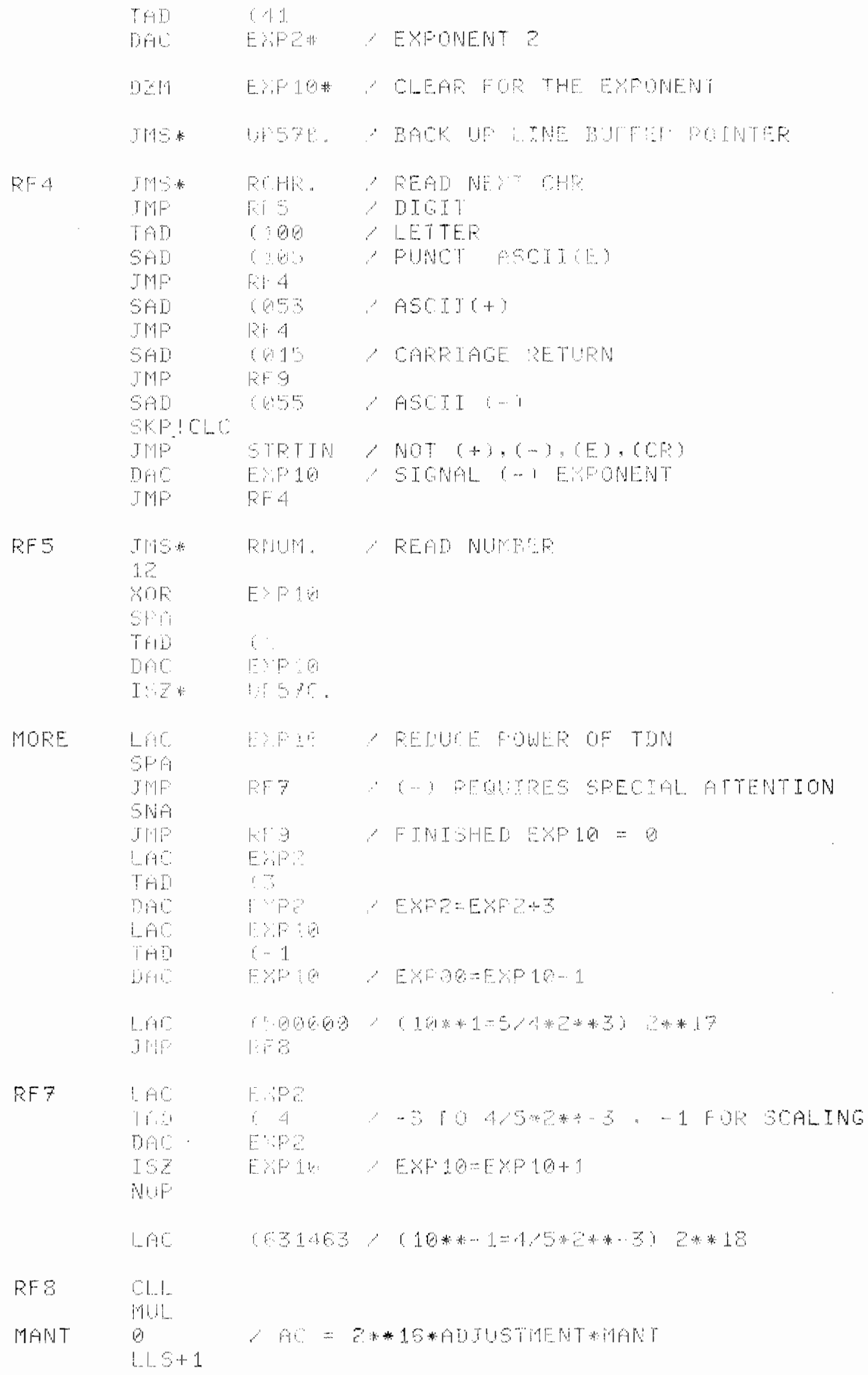




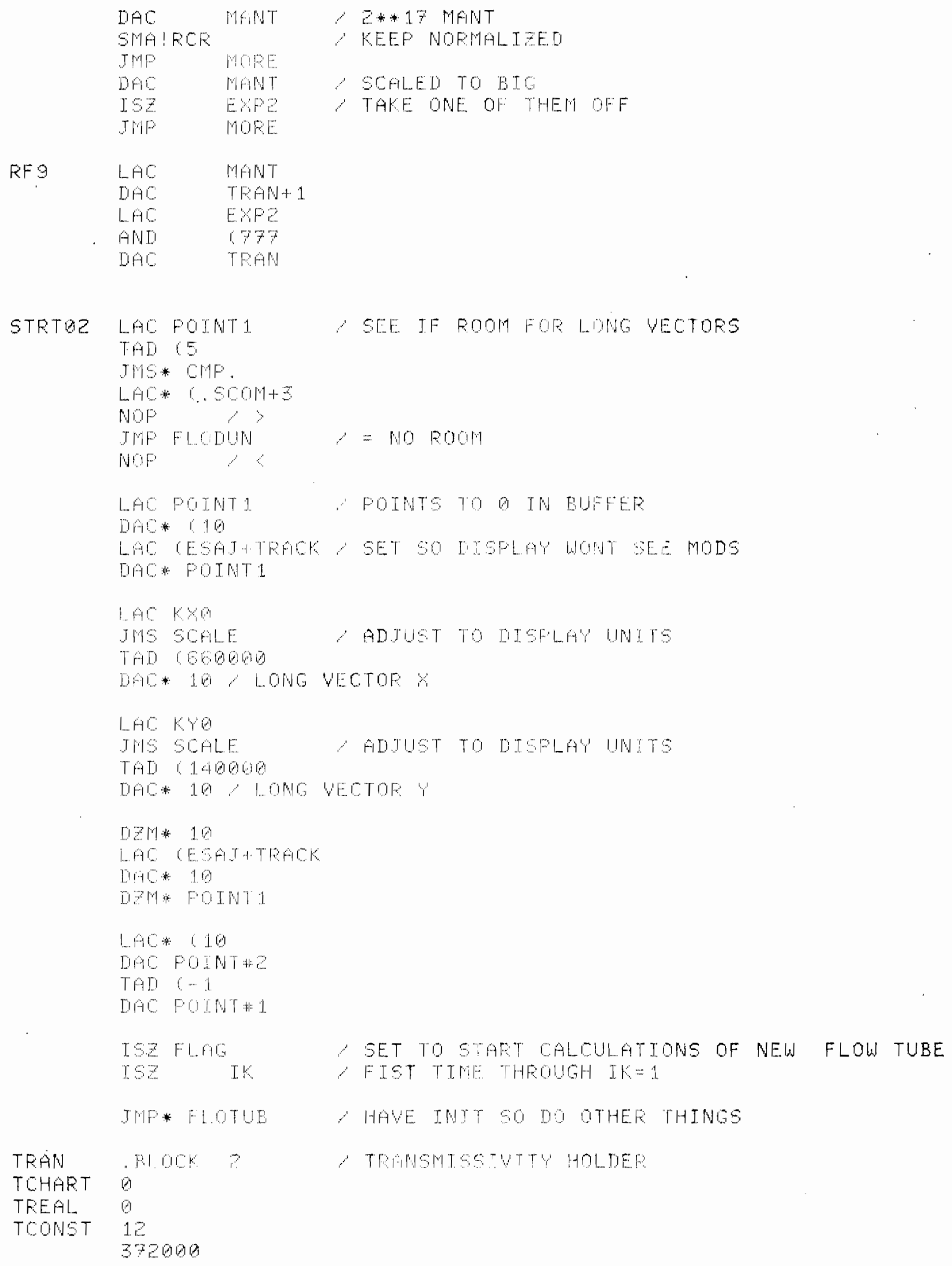




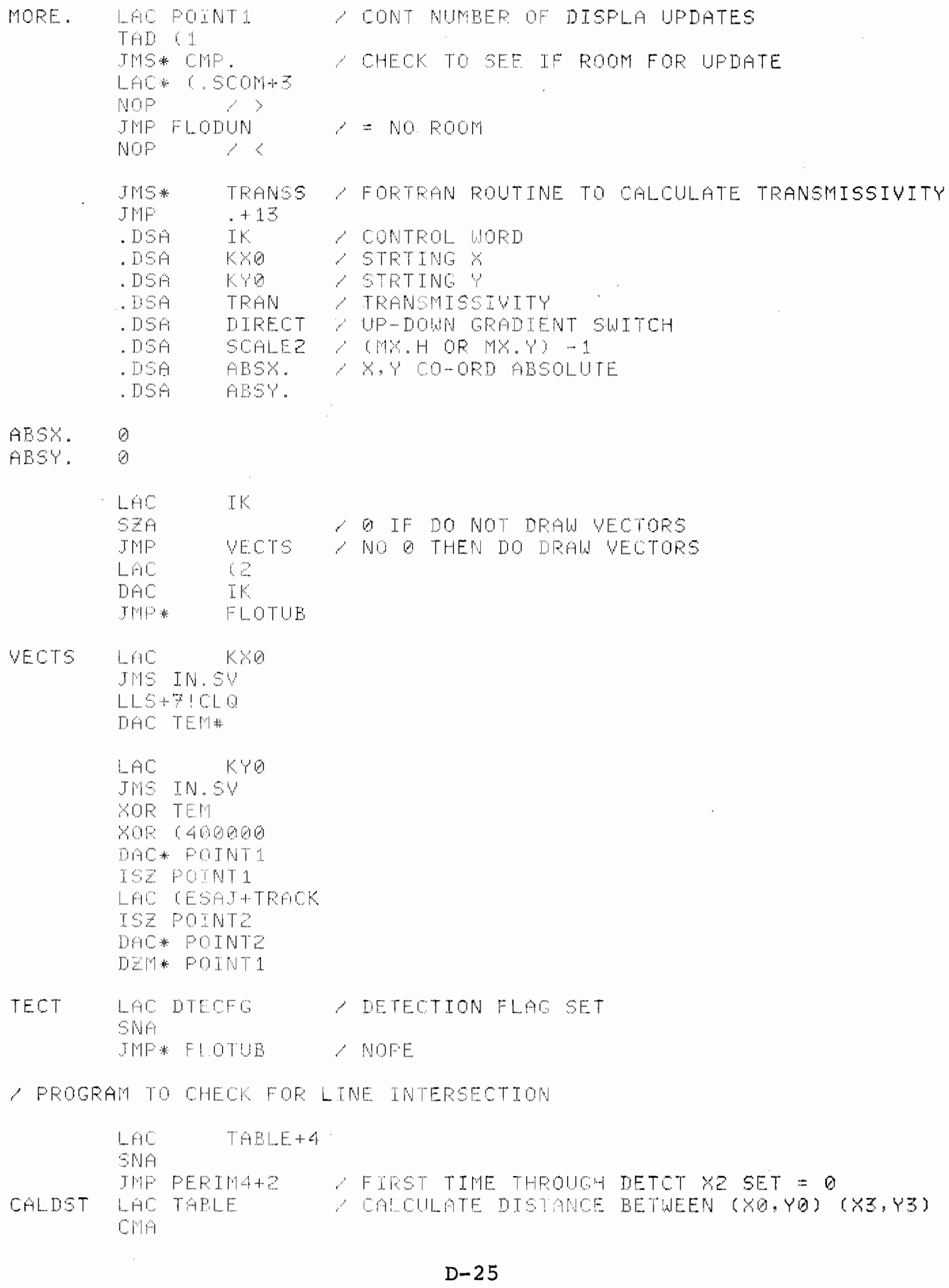




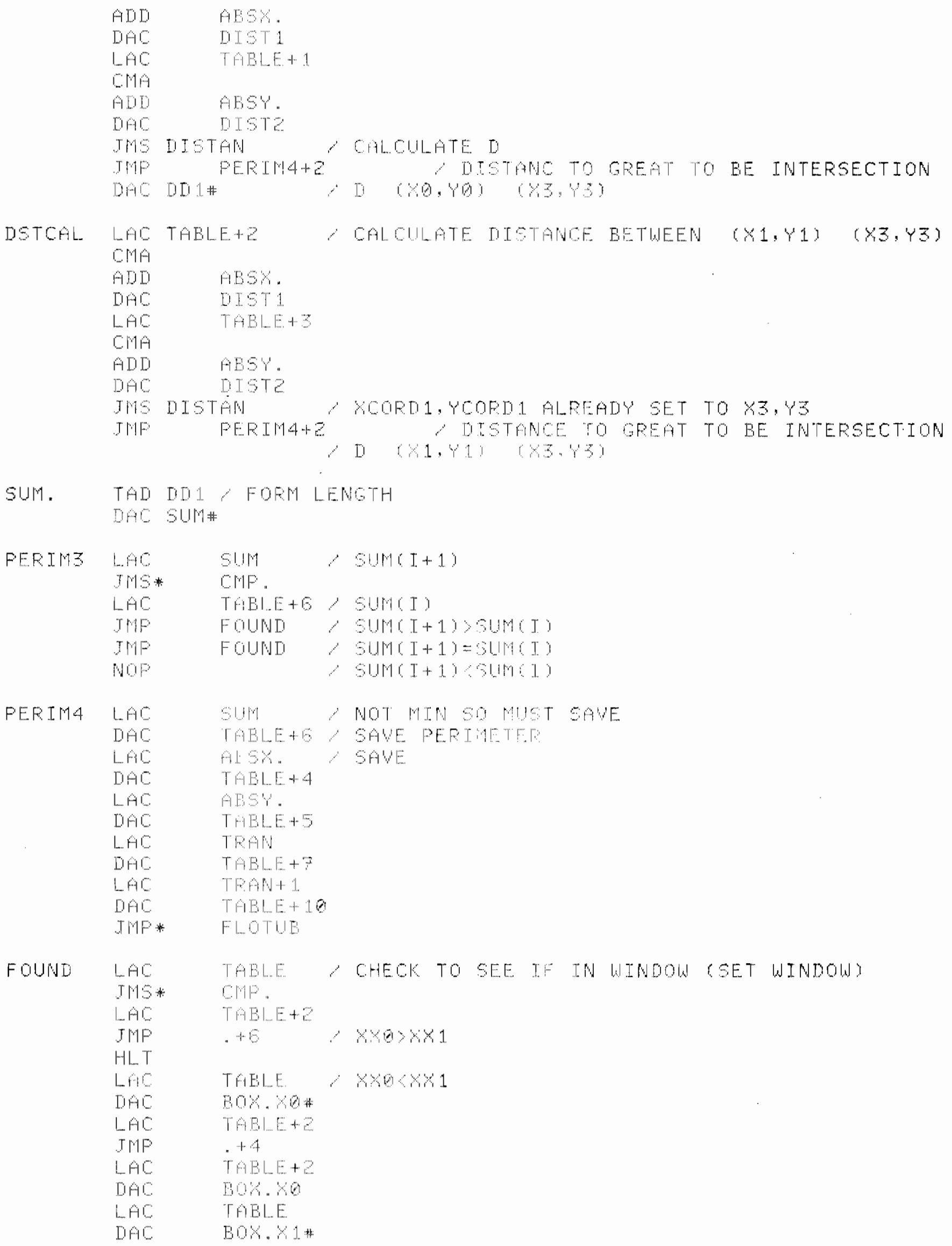




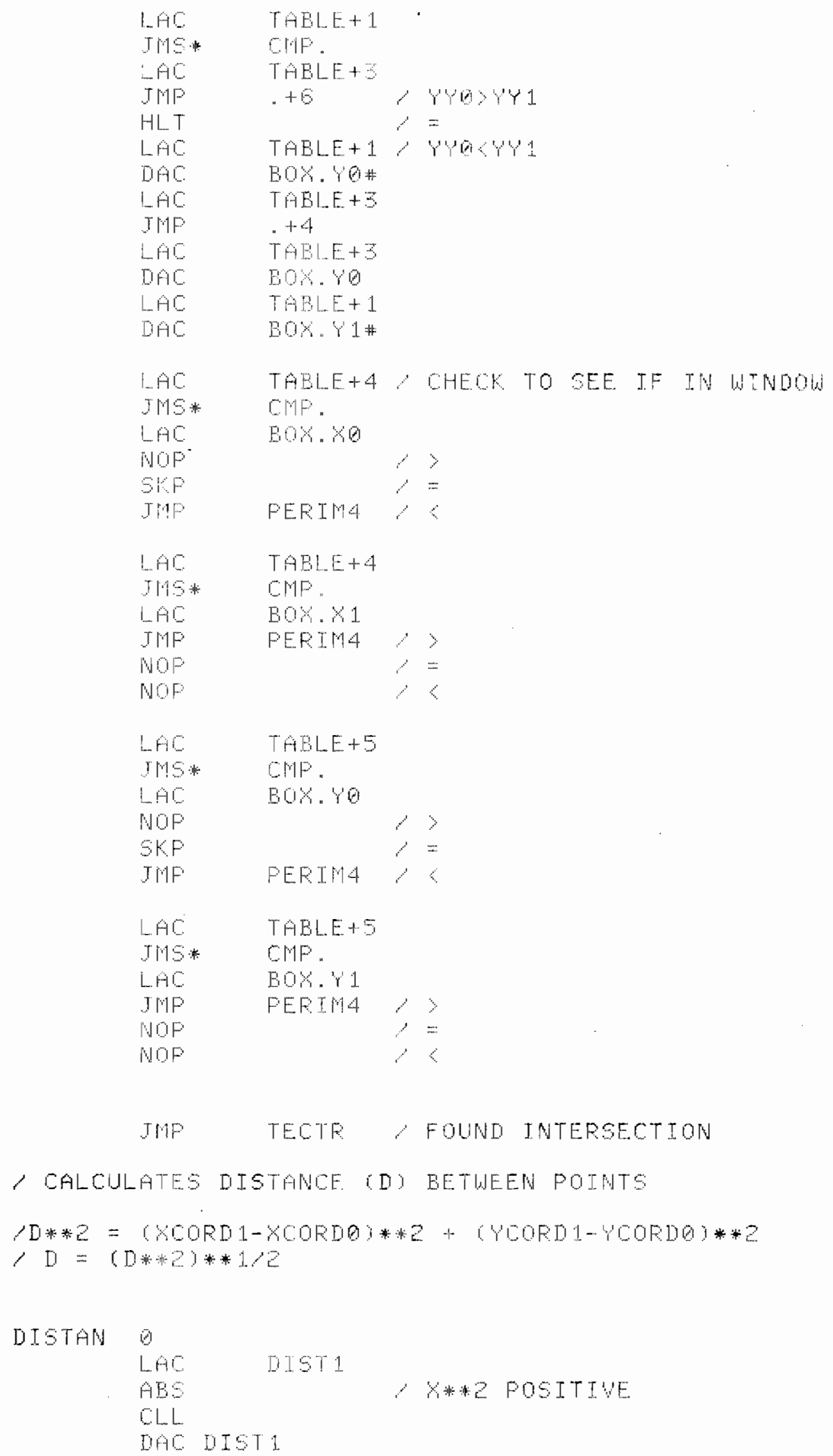




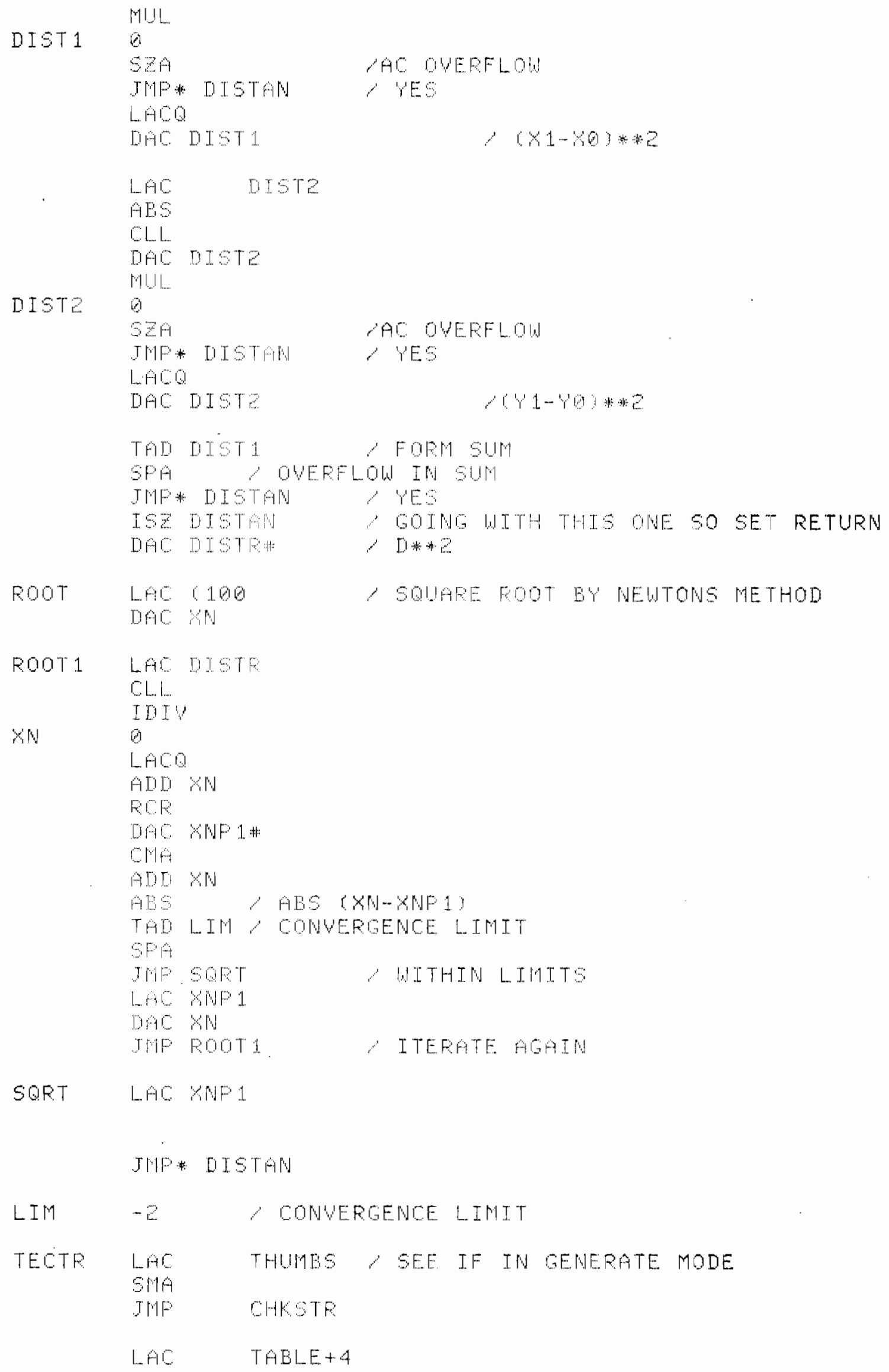




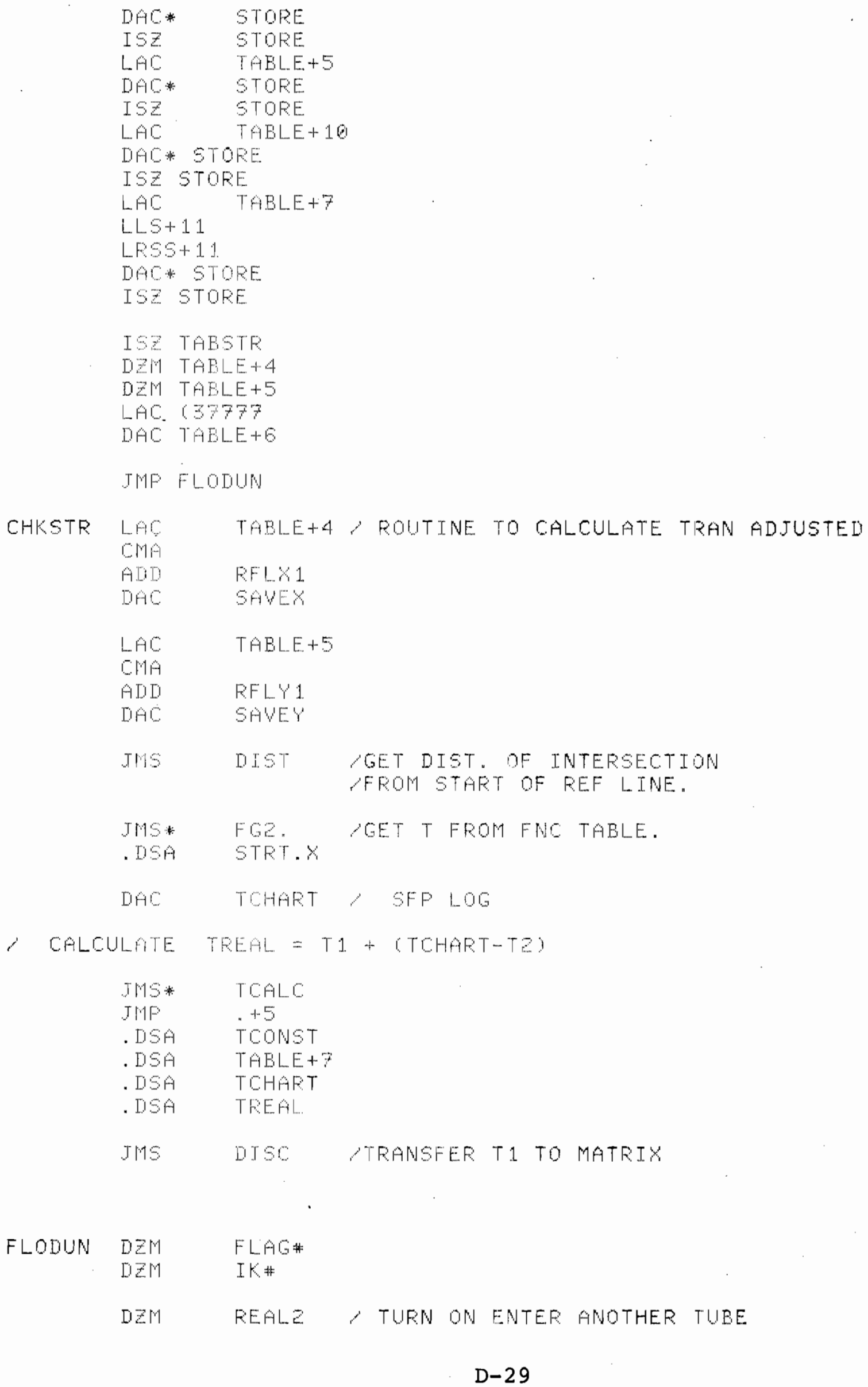




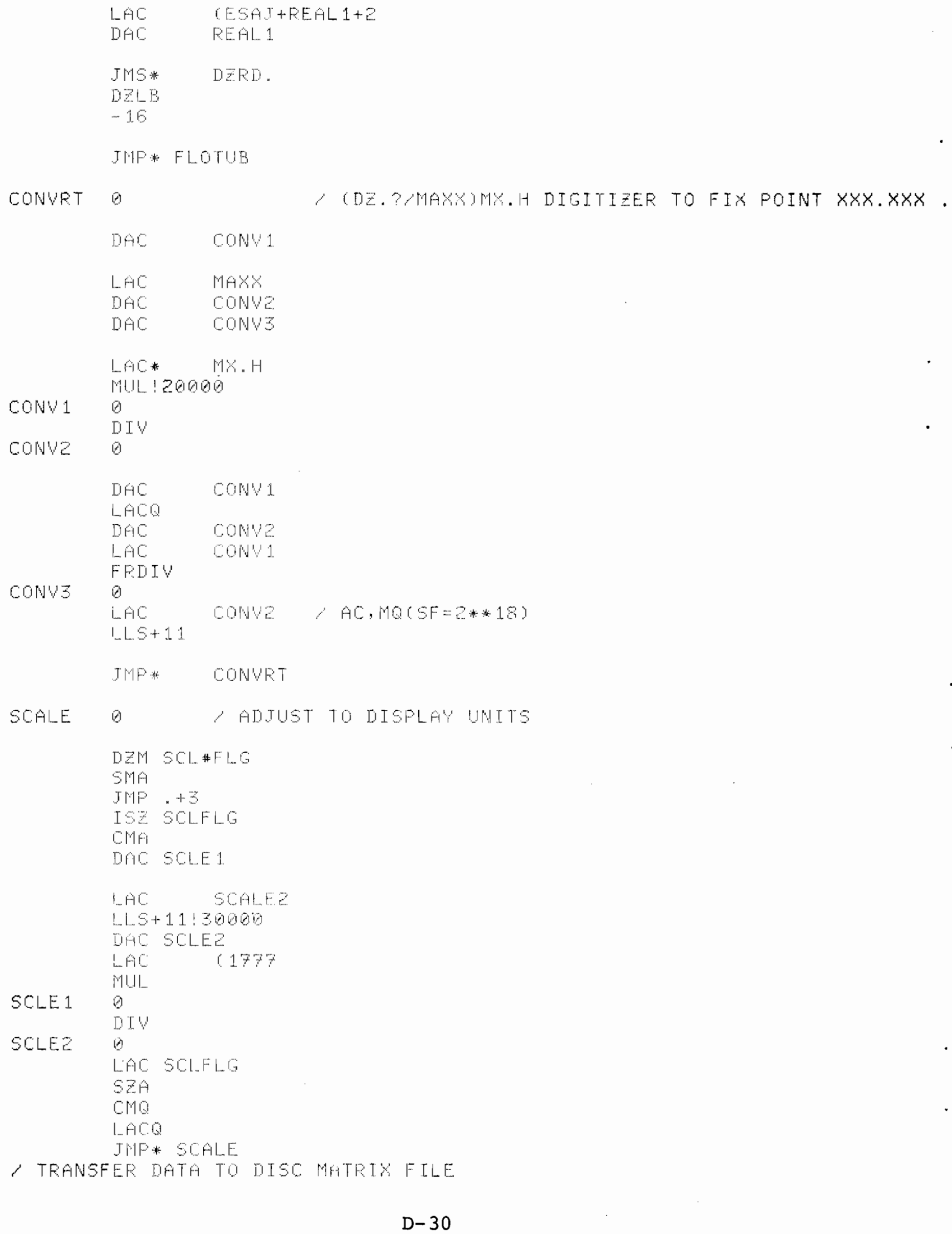




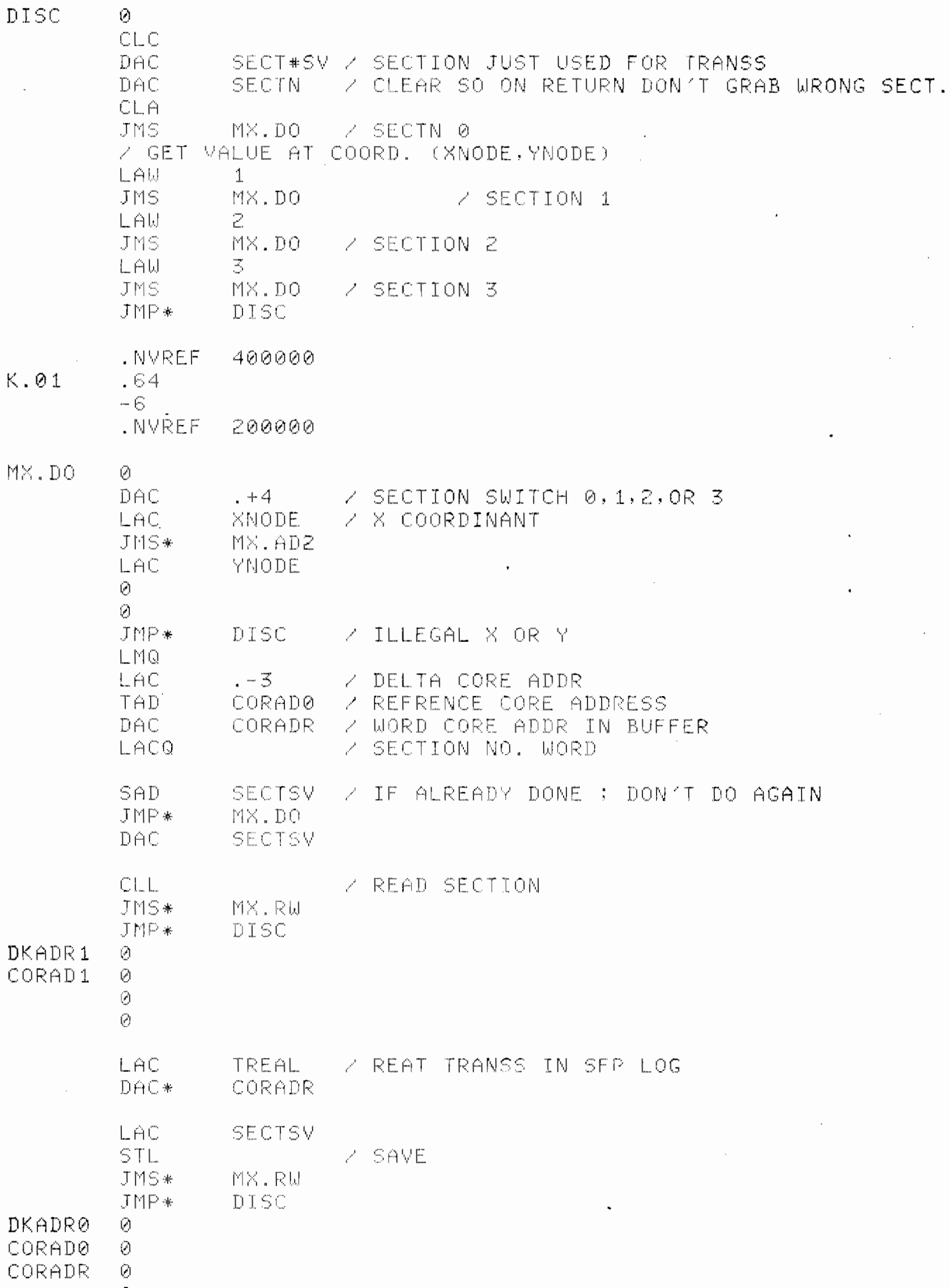




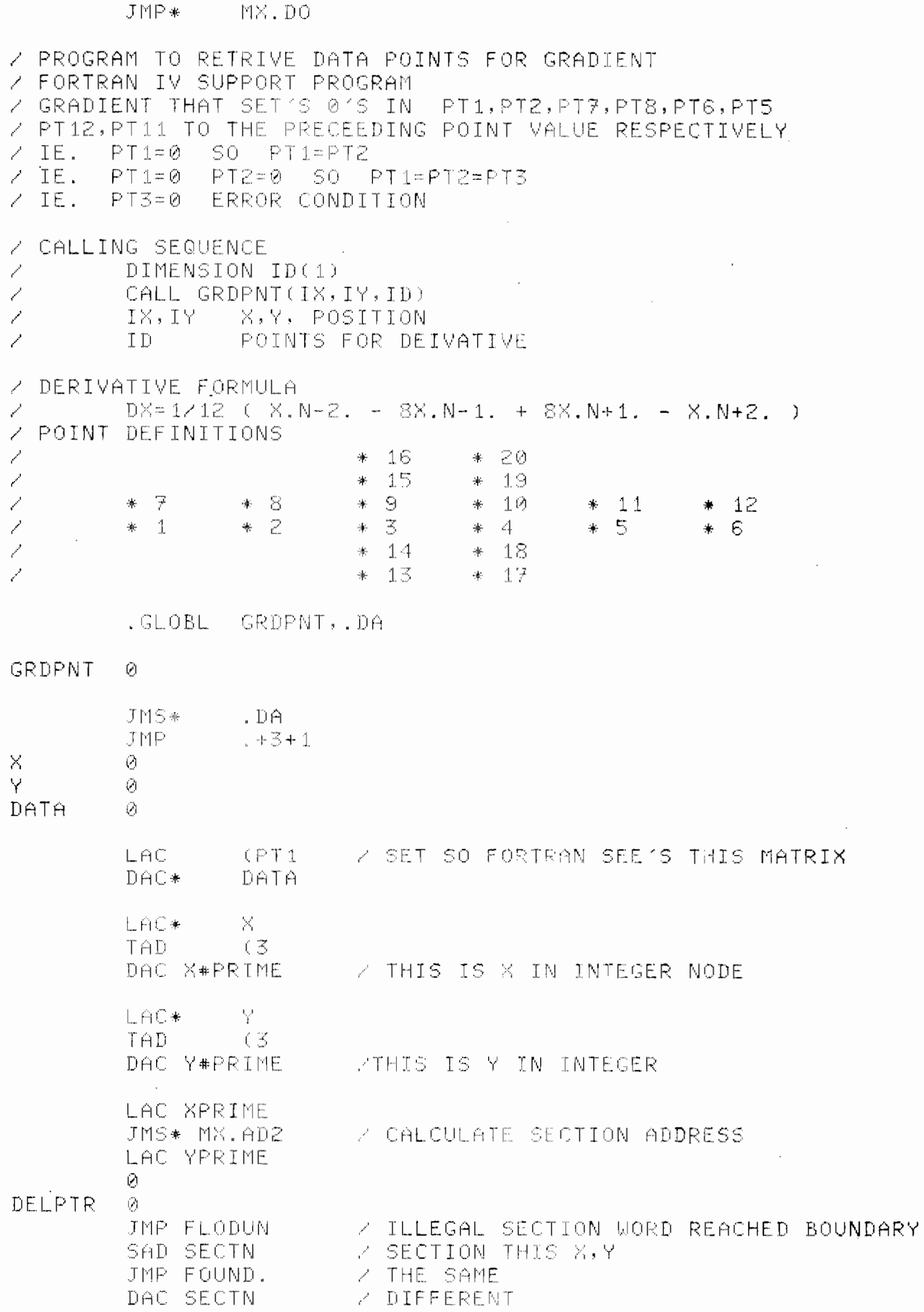




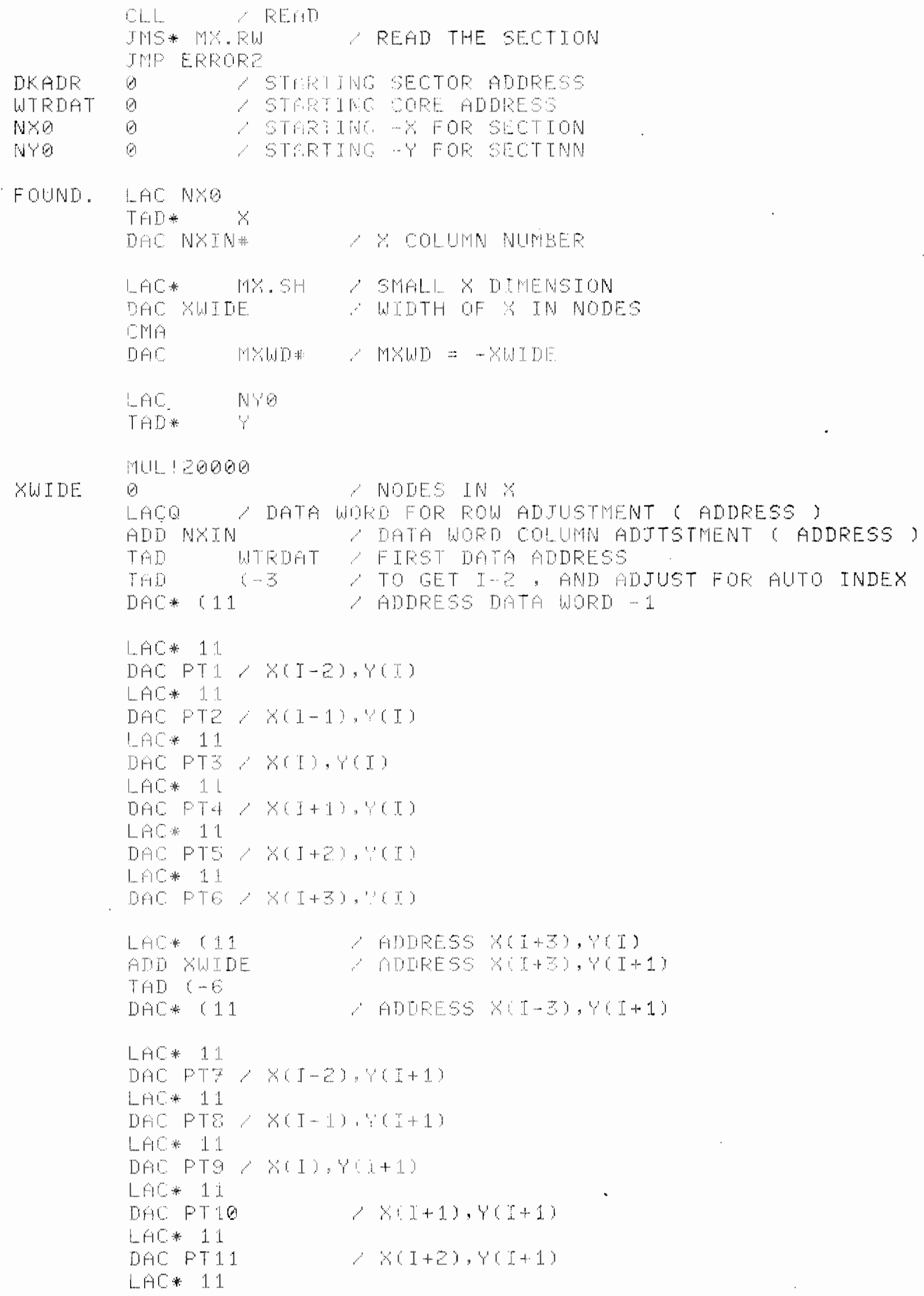




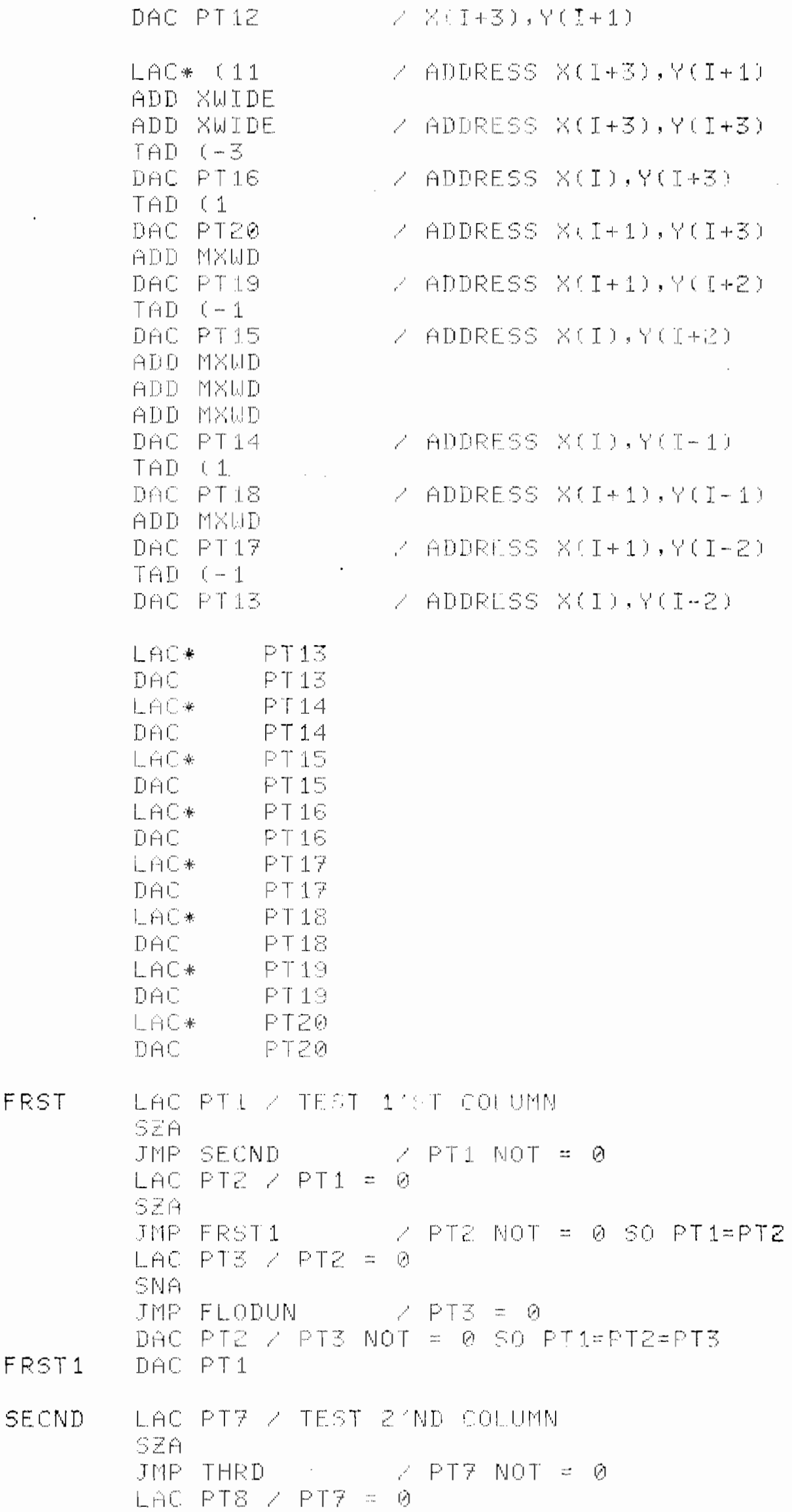




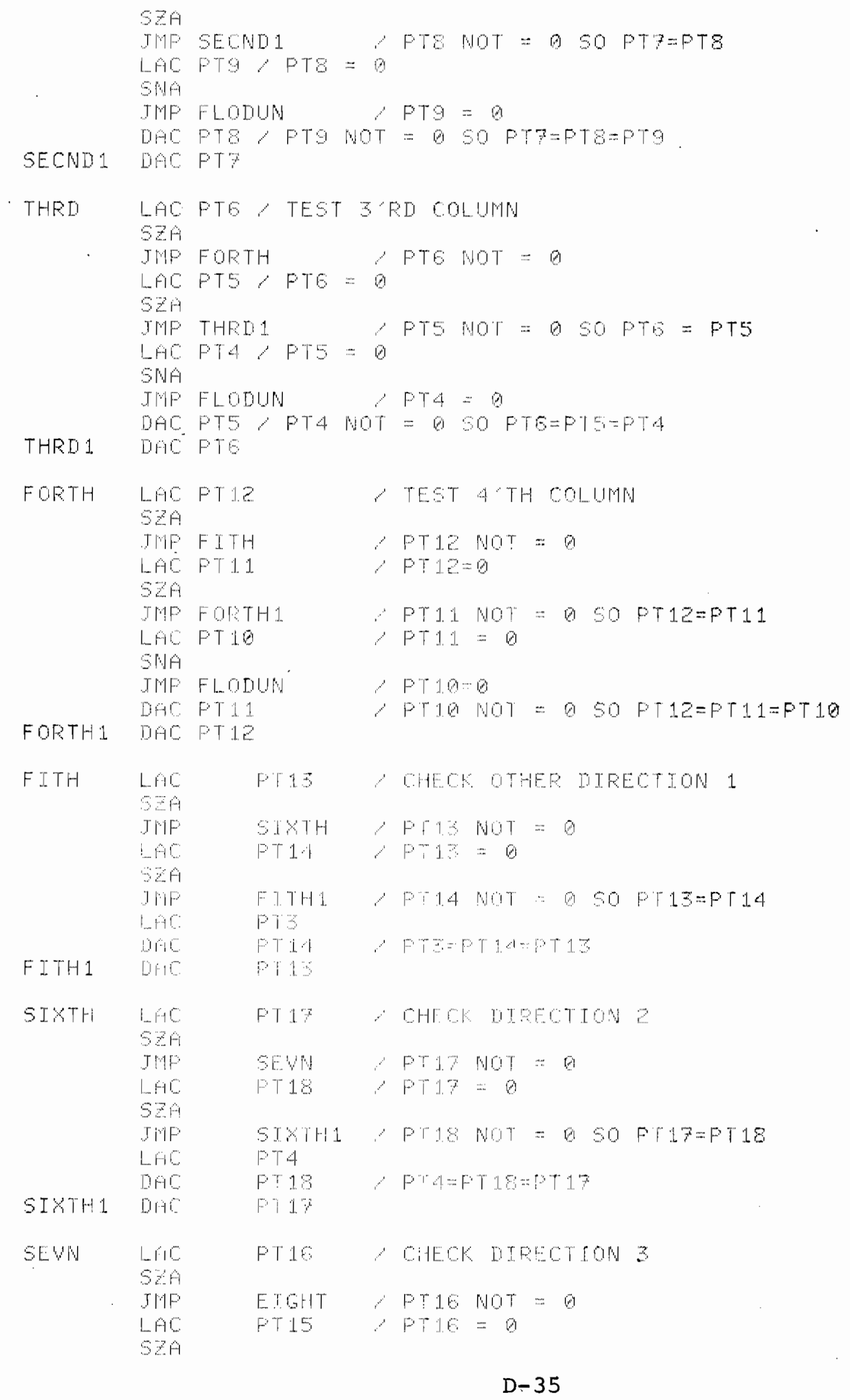




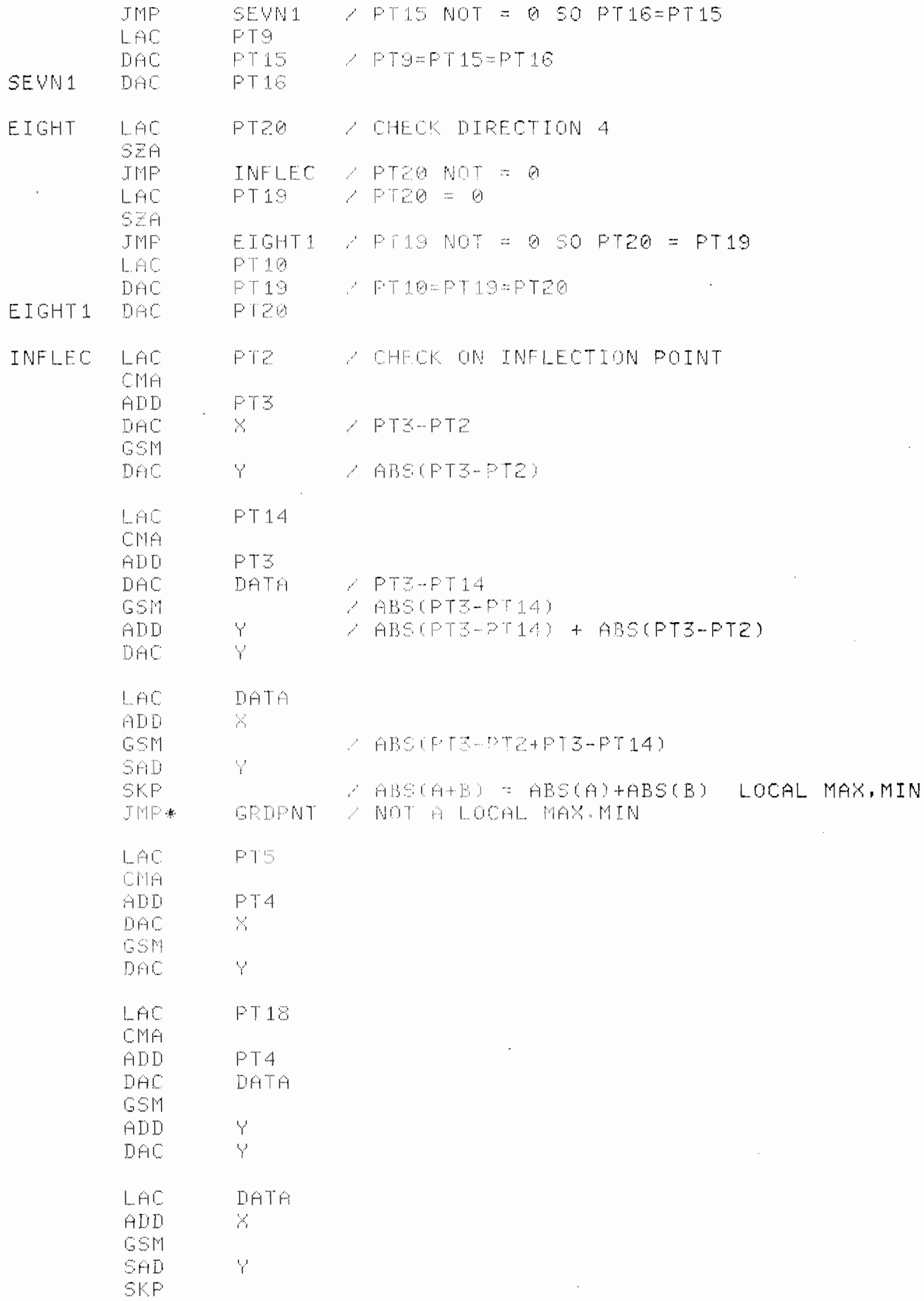




\begin{tabular}{|c|c|}
\hline$T M P \cdot N$ & GथDसए \\
\hline $\begin{array}{l}\angle H C \\
M A\end{array}$ & F.19 \\
\hline ADD & PTS \\
\hline Diti: & 6 \\
\hline $5: 19$ & \\
\hline DAC & $y$ \\
\hline $\begin{array}{l}\text { LAC } \\
\mathrm{MA}\end{array}$ & PTLS \\
\hline ADD & PT9 \\
\hline DAC & DATA \\
\hline Gis & \\
\hline ADL & $Y$ \\
\hline DHC & Y \\
\hline LAC & DATA \\
\hline ADO & \\
\hline 6511 & \\
\hline 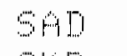 & $Y$ \\
\hline S६P & \\
\hline $\mathrm{T} \mathrm{W}^{\circ}=$ & SWENT \\
\hline$\angle A C$ & FT 11. \\
\hline ADL & PT \\
\hline DAO & $y_{1}$ \\
\hline 69 & \\
\hline DAC & $Y^{\prime}$ \\
\hline LAC & PT 19 \\
\hline $\mathrm{CMA}$ & \\
\hline ADI & FTl \\
\hline DAC & DคТА \\
\hline $65 \%$ & \\
\hline ADL & 'Y' \\
\hline DAC & $Y$ \\
\hline $\begin{array}{l}\angle A C \\
A D D\end{array}$ & DATA \\
\hline $\begin{array}{l}\mathrm{ADDT} \\
6 \mathrm{WM}\end{array}$ & \\
\hline $59 \mathrm{AL}$ & $r$ \\
\hline SК & \\
\hline$\left|W^{\prime}\right| F^{\prime}$ & GFOPUT \\
\hline 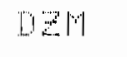 & $\mathrm{r}$ \\
\hline LAC & FTE \\
\hline $\mathrm{CHA}$ & $10=$ \\
\hline $\begin{array}{l}A D D \\
65 \%\end{array}$ & 113 \\
\hline $57 \mathrm{~L}$ & \\
\hline $15 z$ & $x$ \\
\hline $\begin{array}{l}\text { LAC } \\
\text { CHA }\end{array}$ & PTS \\
\hline
\end{tabular}




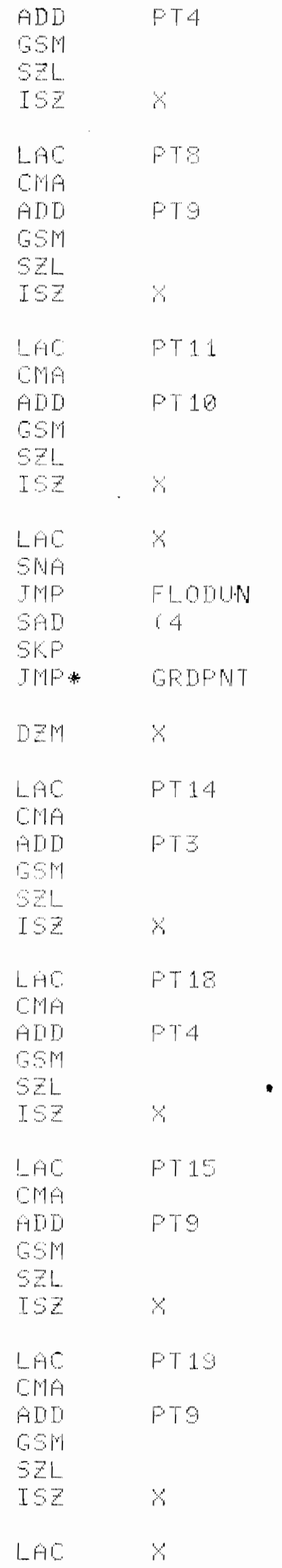


SNA

JMF FLODUN

SAD $\quad$ (4

JMF FLODUN

JMF* GRDPNT

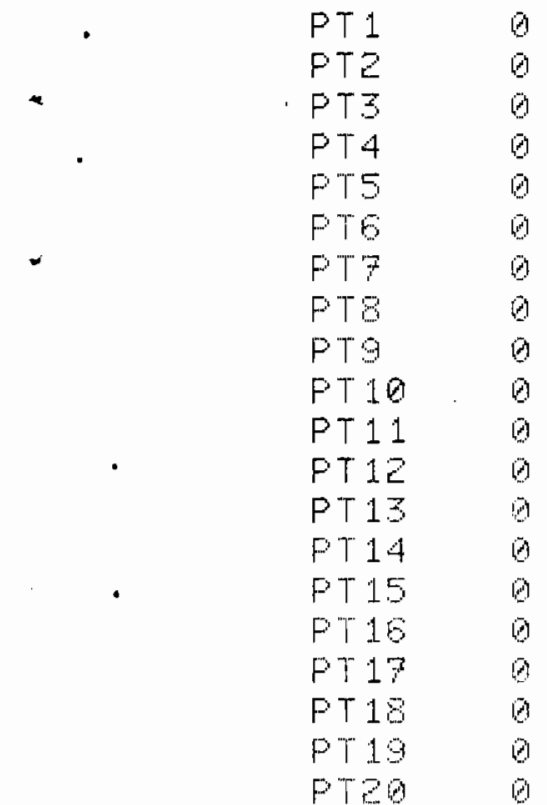

TRACK ESAT+.+1

681000

141000

030000

ESAT+TRACK 1

TRACK1 48E I20

40400

402010

490020

422010

424000

422110

400120

402110

920000

442000

400040

454120

410000

020000

TEACKE ESAT+SETZ

'

SMALL TRACKIMG CROSS

I

TPACK $\quad 461110$

402000

401004

400010

421004 


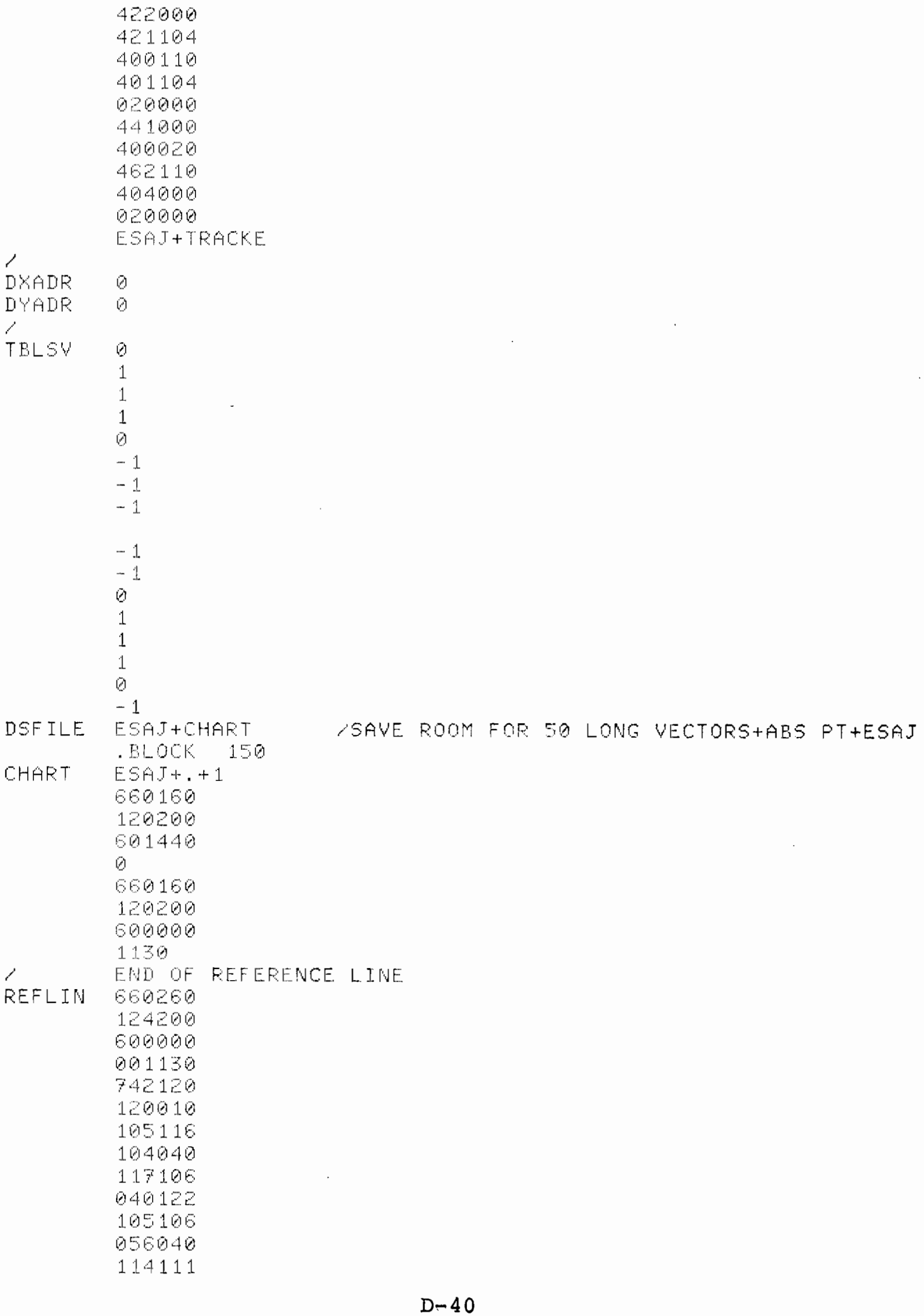




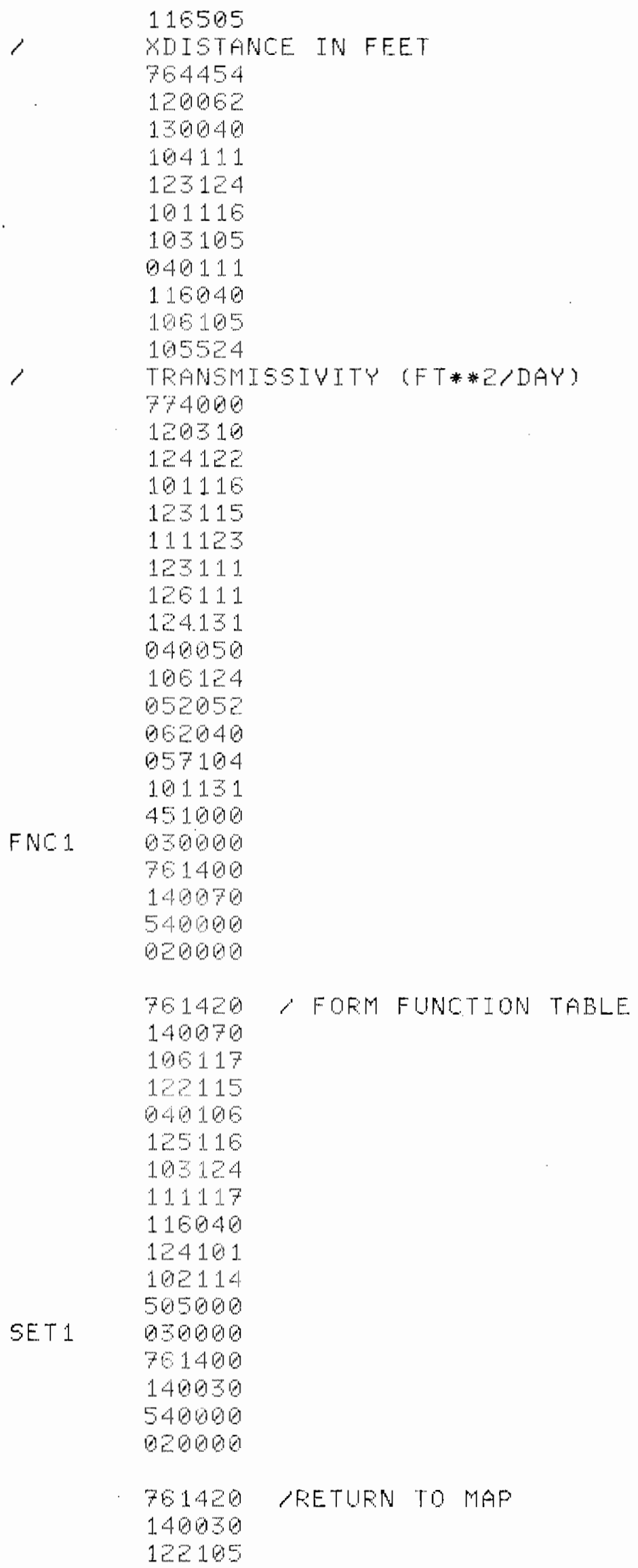




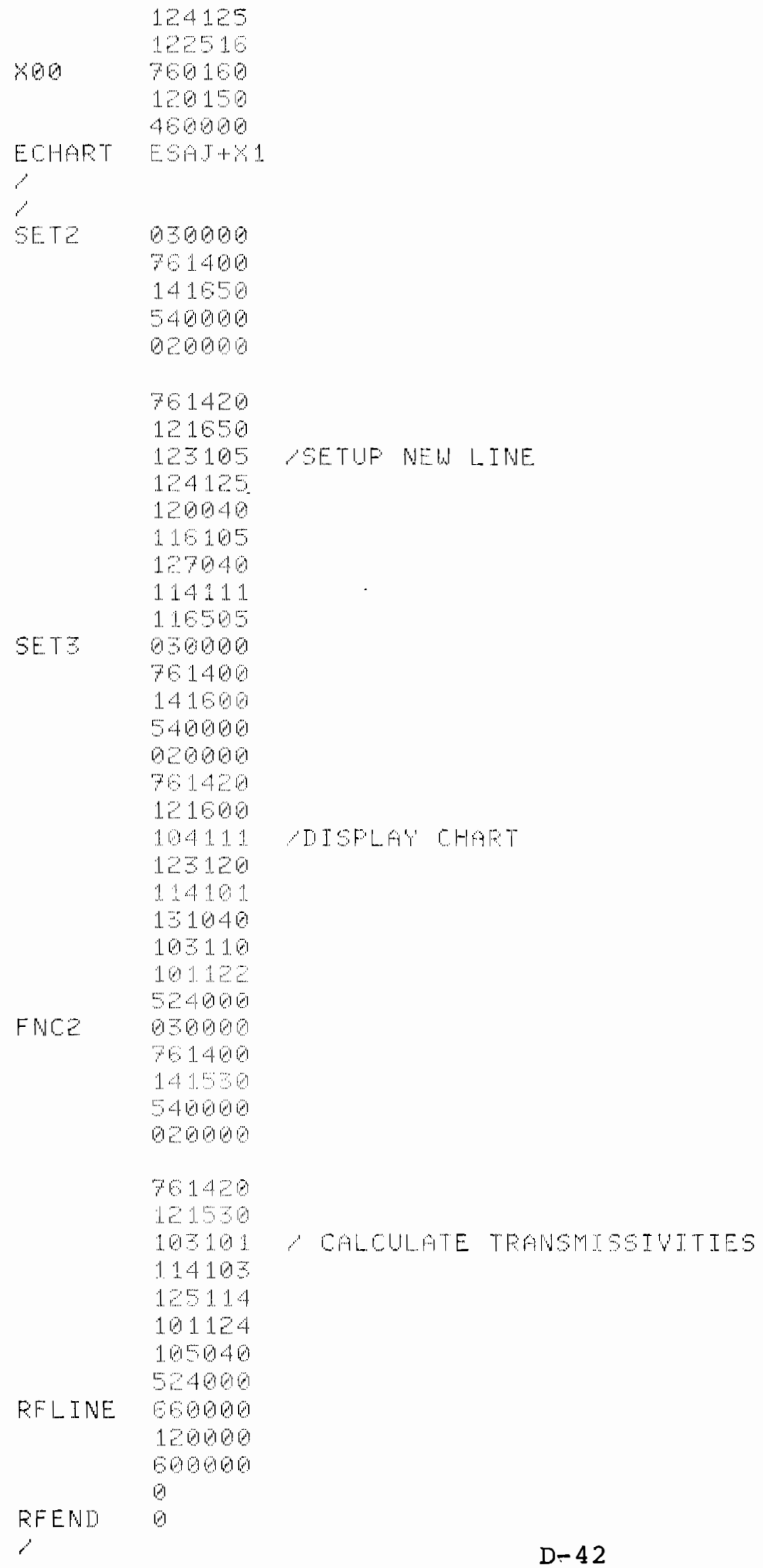




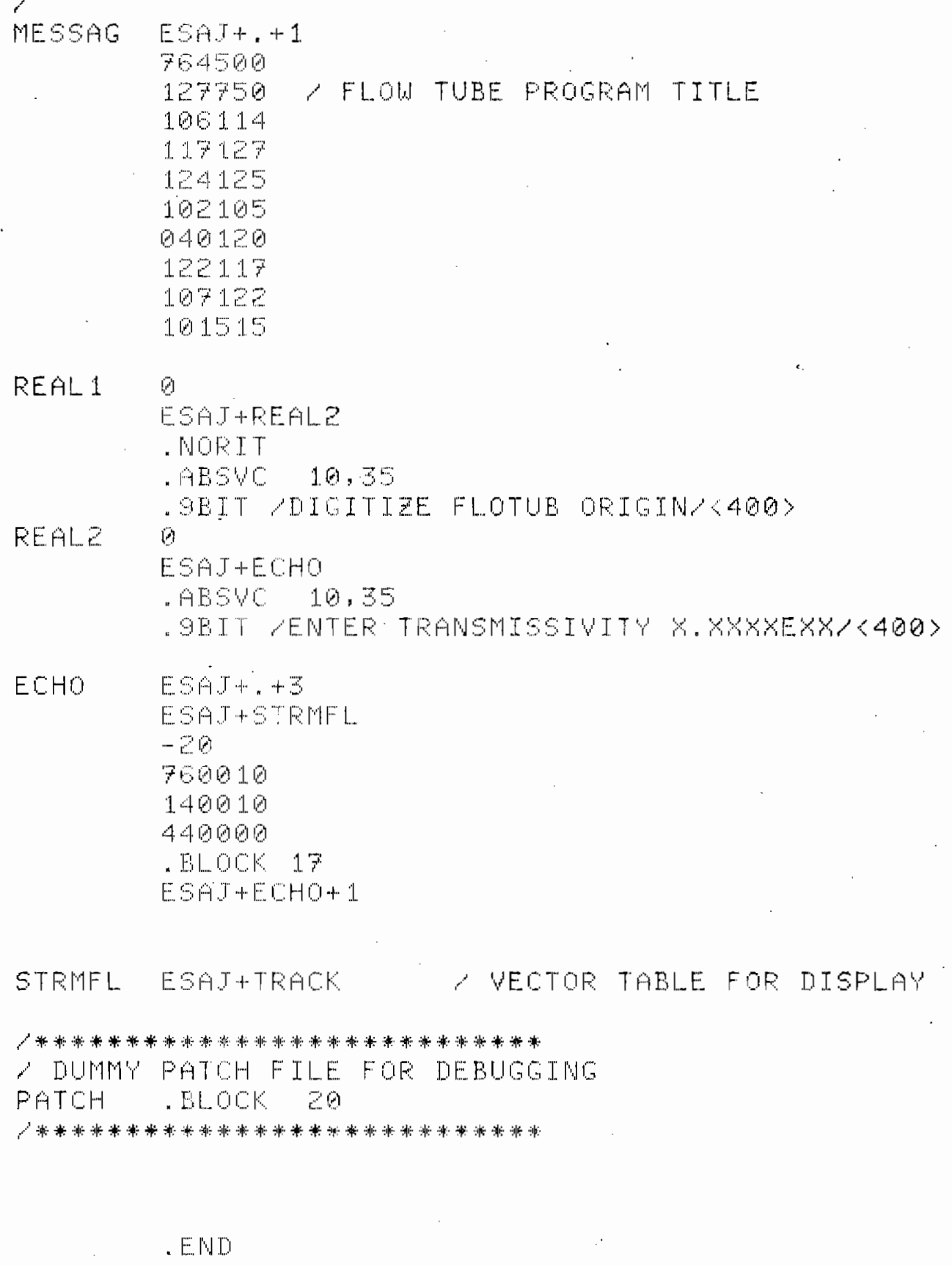


SUBROUIME GFADSSY Y,H,GX, GY, GRADI

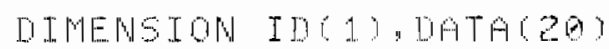

EQUTYALENE (PT 1, DATH(1) , PTZ, DATA(Z)), (PT3, DATA(3))

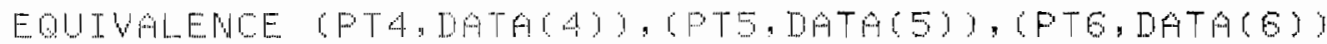

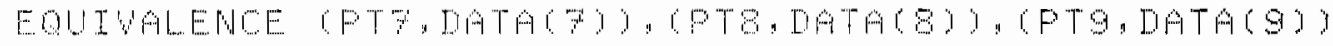

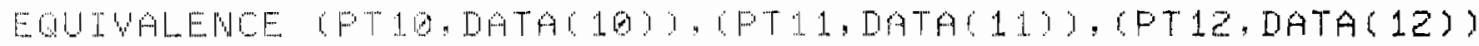

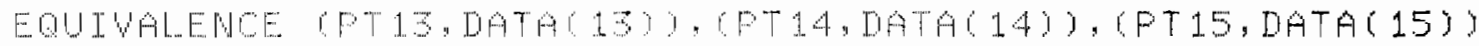

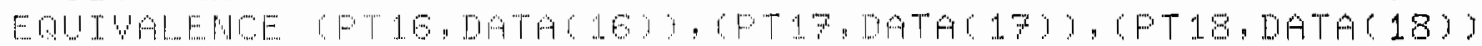

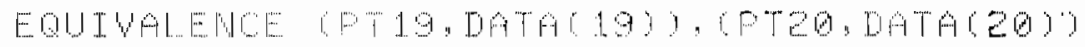

C

I $Y=$ IF TSPY

IY= IFIXIY)

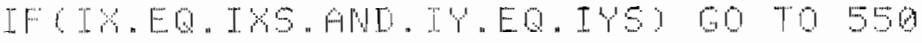

$I \times S=\operatorname{IY}$

I. $Y S=I Y$

C

CAILL GEDPNTIY, IY, ID?

\section{6}

C.

$50500 \quad I=1,20,1$

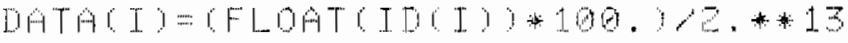

CONT THUE

C

$F Y=X-F L O A T$ I I S

$F Y=Y-F$ LOATCIY'

$G P 1=P T 1-8 .+P T 2+8 . * P T 4-F T=$

DPE=PTE- A ATS-B. ATS-PTS

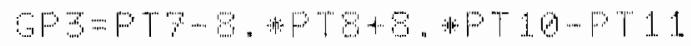

C

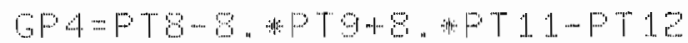

L

$G F 1=G P+F Y \cdots(G F-G P 1)$

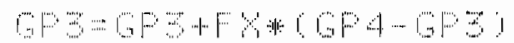

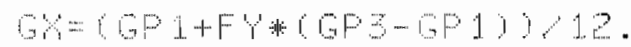

GPL $1=P T 13-8 . * F T 14+8 . * F T 9-P T 15$

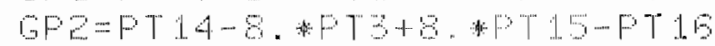

GPS $=P T 17-8$. *PT 18+8.*FT10-PT19

$\mathrm{c}$

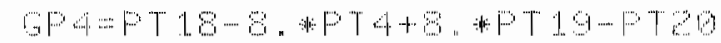

c

$G P 1=G P+F Y=6 P P-G P \perp$

GPE-GFS+FYA $6 P+-G P 3)$

6

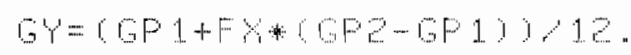

C

GPAD=GYHE+GY*

GRAT= SOPTEGRAD:

GP1=PT3+FX 1 PTA-PT3!

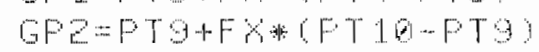

c

$H=G P 1+F Y$ W $(G P E-G F 1)$

RETURW

END 
SUBROUTINE TRANGSTIK, IY, IY, TRAN, ITCH, IV, IAX, IAY)

c

C

C.

C

c

C.

$\square$

5

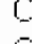

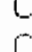

c

C

C

0
0
0

0
0
0
0
0

C.

$100 \quad W N=W N P D$

TH:= TRAW

GEADW = GRDUP

$c$

$K M D=K X$

$K Y Q=K Y L$

$x Q=x 2$

$Y Q=1 \%$

$x 1=x 3$

$y 1=Y 3$

C

CALL GRDENT YYOYY, GX, GY,GRADY

$\mathrm{C}$

C

$X 2=x Q+(G \times Y G E A D)+S T E P$

$Y Z=Y Q+6 Y, G R A D Y S T E P$

r:

GALL GRDEHTCY1,Y1, GX, GY, GRAD

$x 3=x 1+6.6 \% 6 R A D)$ STEP

$Y S=Y 1+C$ GYGPADISTEP

C 


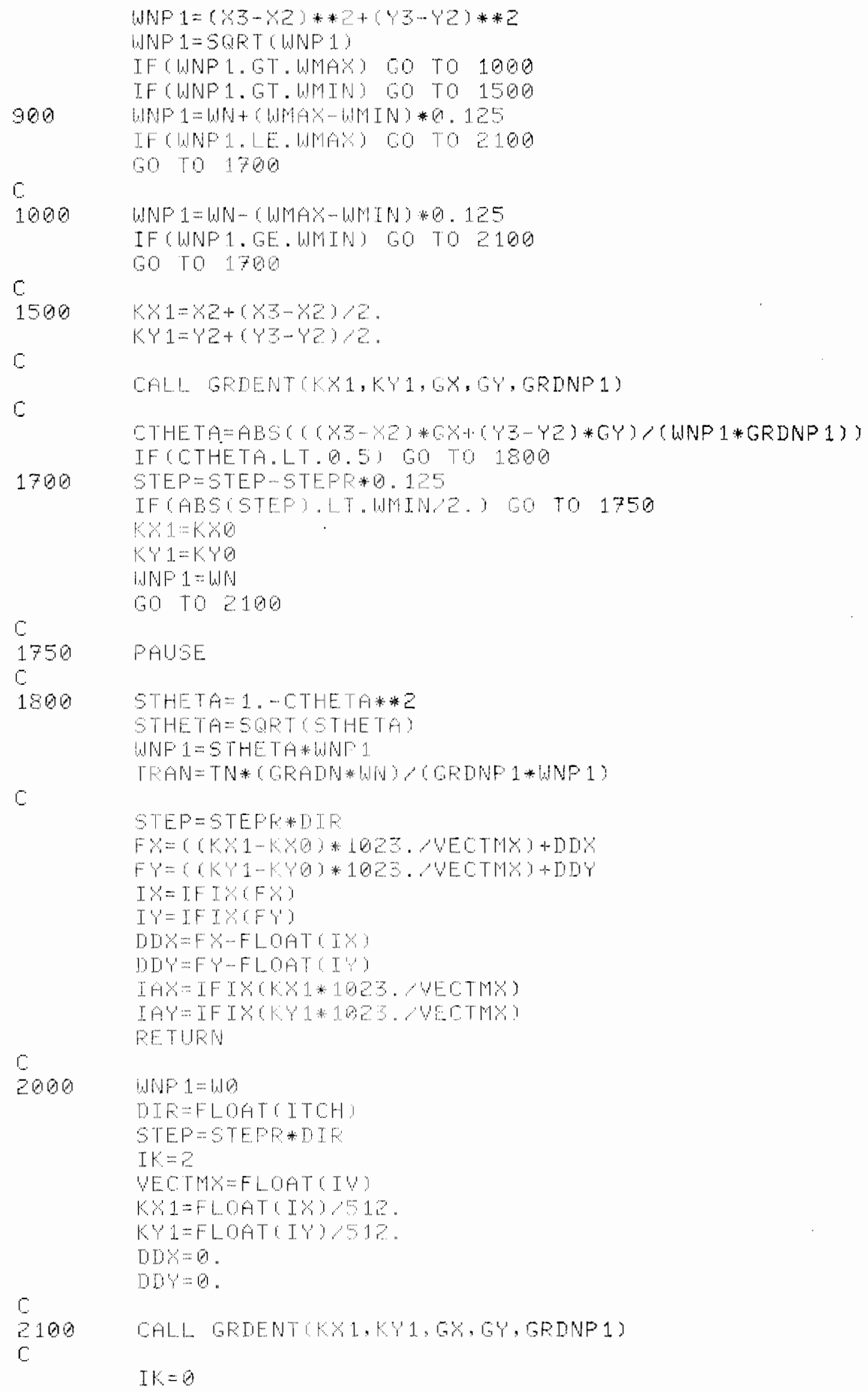


c

DY= WNF $1 \%$. WGR GPDNF 1 * DIR

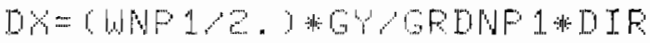

C

$2200 \quad x 2=K Y 1+I X$

$Y 2=K Y 1-D Y$

$\mathrm{X} Z=\mathrm{R} \times \mathrm{B} \mathrm{DK}$

$Y Z=K Y 1+D Y$

6

RETURP

C

END 


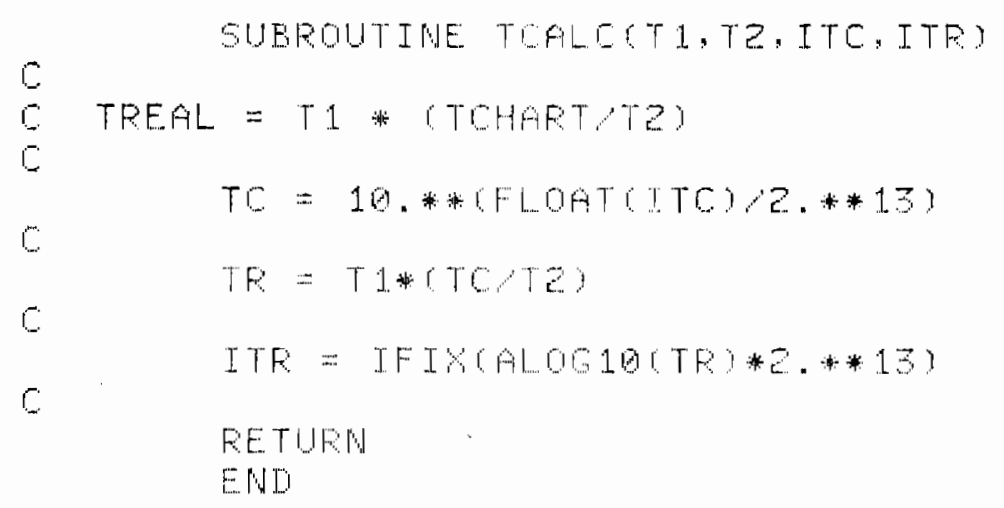


APPENDIX E

TRANSMISSIVITY PROGRAM (TRANSIENT STATE) LISTING 


\section{TRANSMISSIVITY PROGRAM (TRANSIENT STATE)}

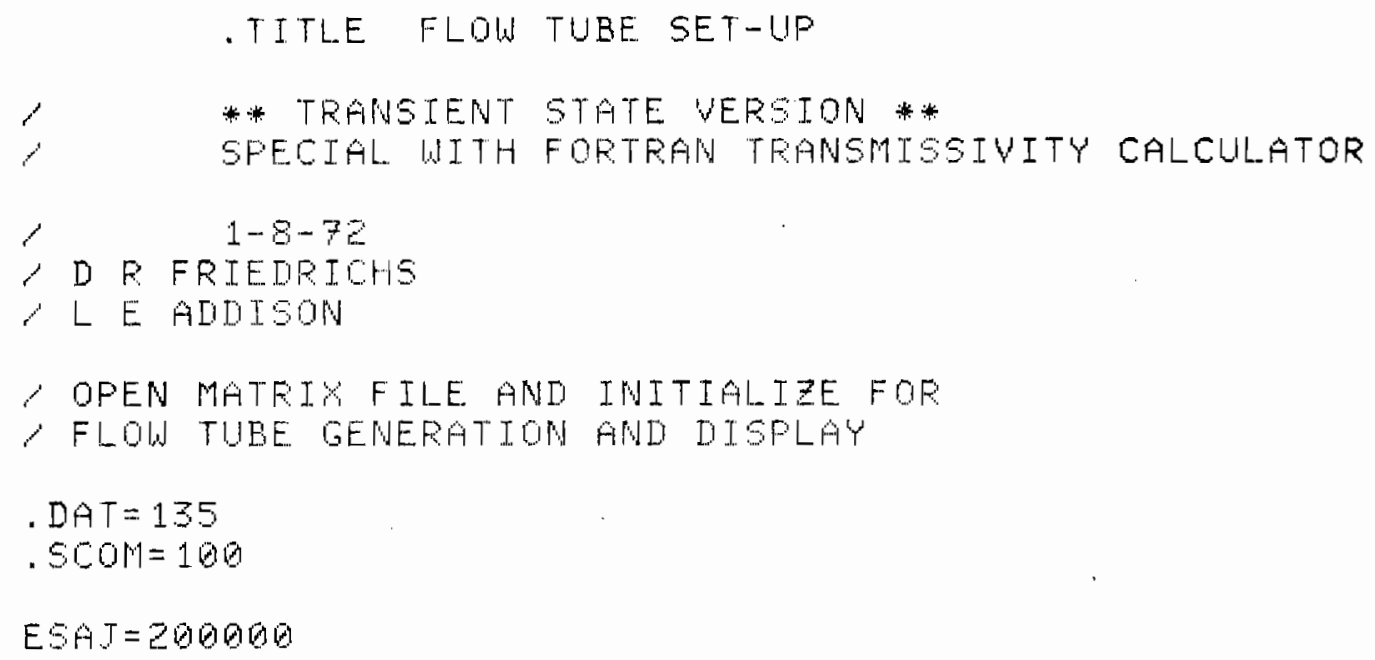




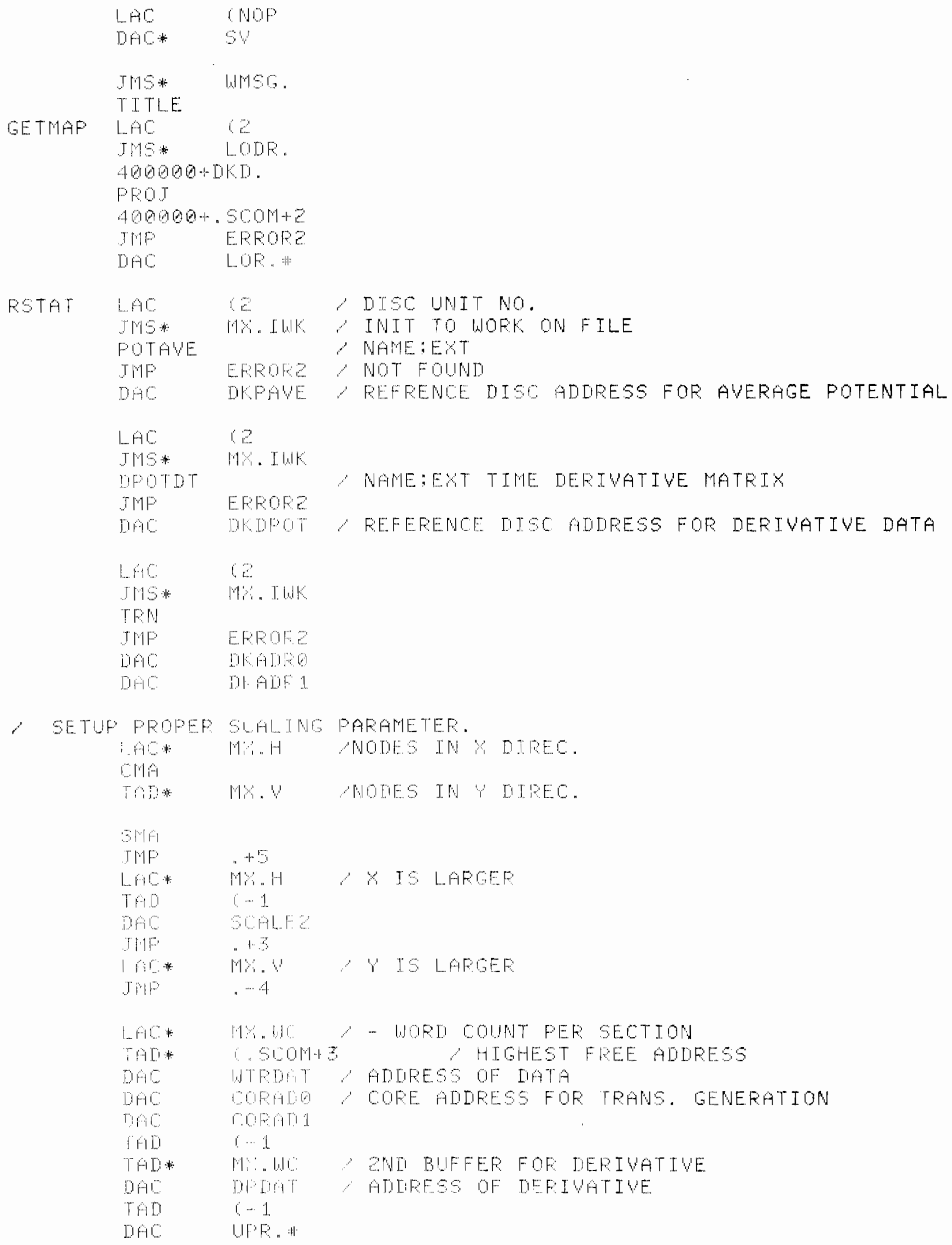




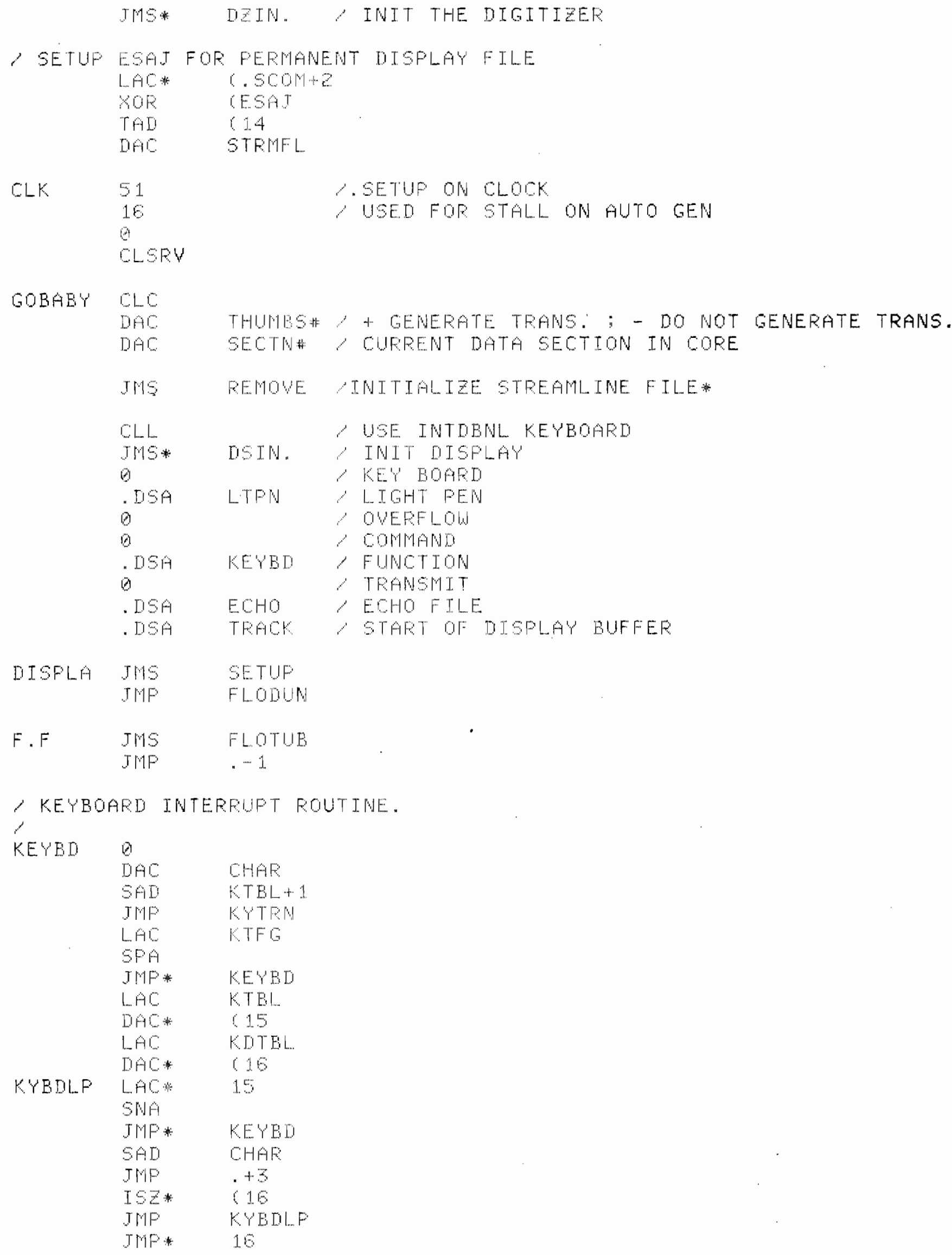




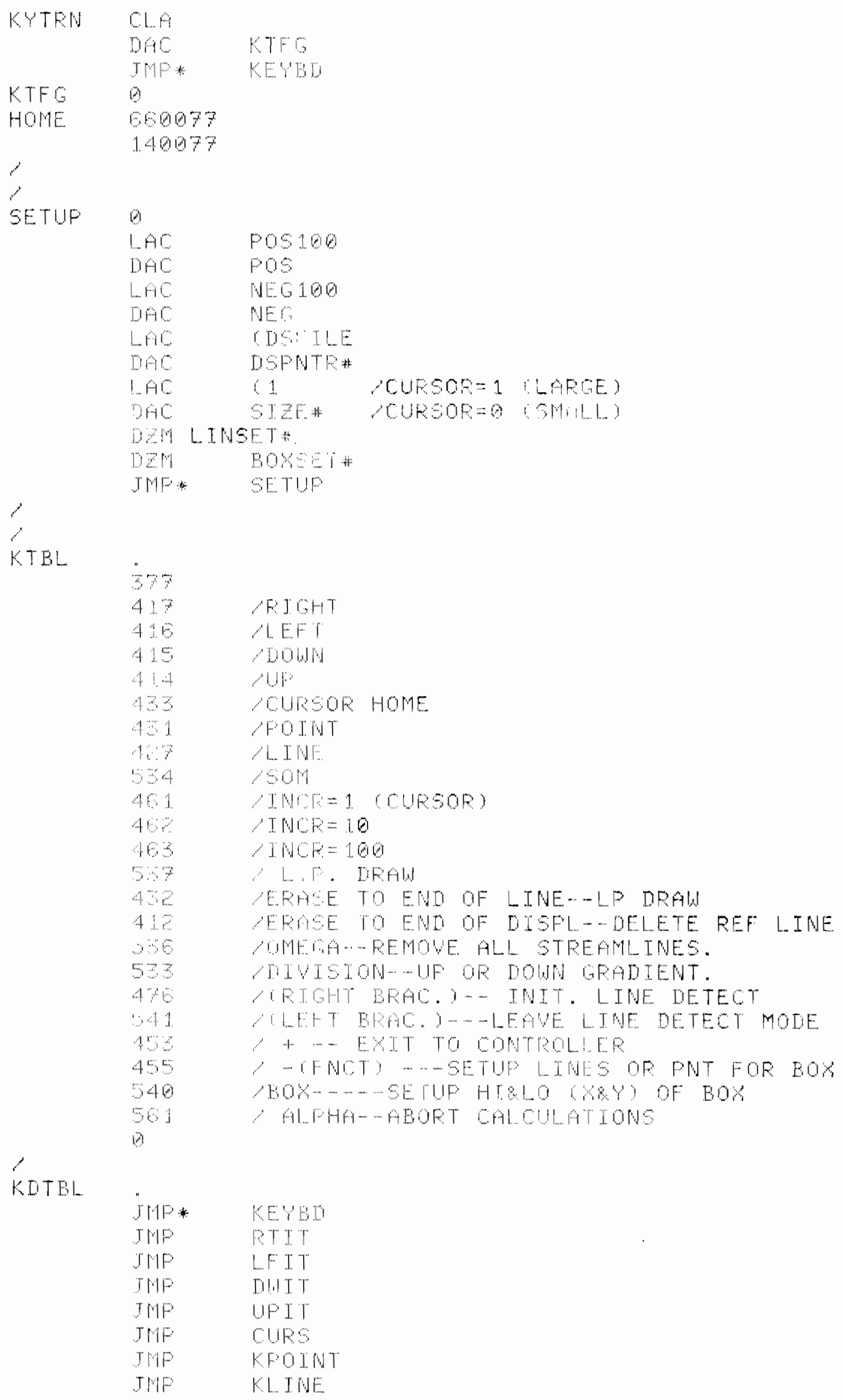

KTBL

37

417

46

415

4

4

1

11,7

56

461

46

46

5

42

41

256

53

4 ;

31

1

45

30

561

i

\begin{tabular}{|c|c|}
\hline TF* & Bu \\
\hline $\mathrm{T} M$ & TT T \\
\hline $5 \%$ & L...F T T \\
\hline JीP & {$[1,1 \mathrm{~T}$} \\
\hline$T " 1 \mathrm{~F}$ & UPIT \\
\hline $79 F$ & curs \\
\hline$T \mid W$ & GFOT \\
\hline TWF & KL T IUE \\
\hline
\end{tabular}




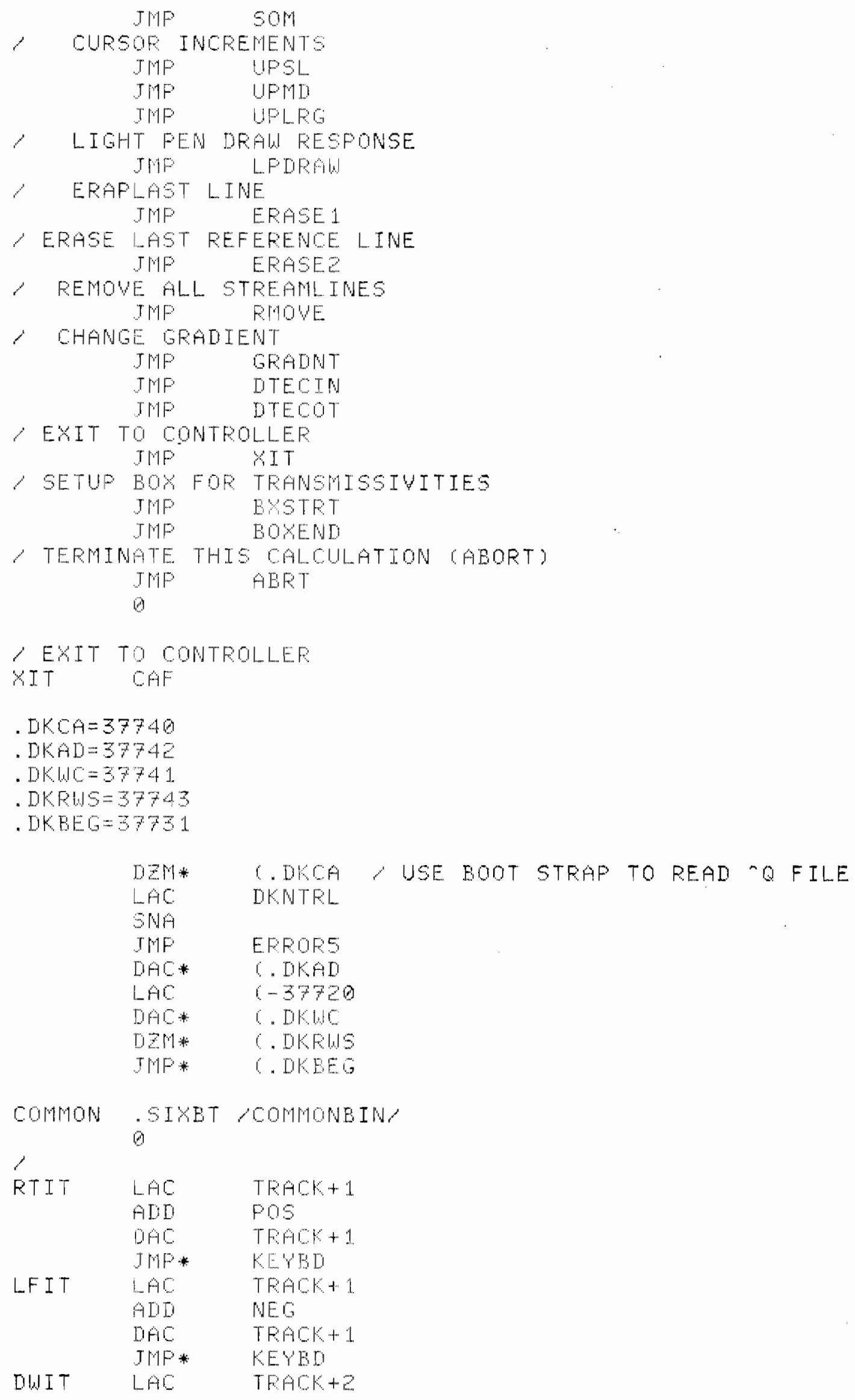

DWIT LAC TRACK+E 


\begin{tabular}{|c|c|c|c|}
\hline & ADD & NEG & \\
\hline & DFE & TPACF+2 & \\
\hline & TMP* & KEYBD & \\
\hline \multirow[t]{4}{*}{ UPIT } & LAC & TRACK+Z & \\
\hline & ADD & $\mathrm{Fos}$ & \\
\hline & DAC & TRACK+Z & \\
\hline & $T \|_{P}$ & KEYED & \\
\hline \multirow[t]{5}{*}{ CU\&S } & LAC & HOWE & \\
\hline & 19० & TRACK+1 & \\
\hline & LAC & HOME + 1 & \\
\hline & DAC & TPACK+2 & \\
\hline & $J M F *$ & KEYED & \\
\hline \multirow[t]{8}{*}{ KFOINT } & LAC & TRACK+1. & \\
\hline & $D A C$ & RFL INE & \\
\hline & 9118 & L." & \\
\hline & DAO & EFLKL $\quad \triangle$ STORE & WALUE OF $X(1)$ \\
\hline & LAC & TRACK+Z & \\
\hline & DAC & $F F L I M E+1$ & \\
\hline & $\mathrm{TNS}$ & LY.I I & \\
\hline & DHE & RHLYL YSTORE & YAL UE OF Y' $(1)$ \\
\hline \multirow{19}{*}{ KLIVE } & & & \\
\hline & $\begin{array}{l}\text { LAC } \\
\text { TMS }\end{array}$ & $\begin{array}{l}\text { TrACK + } 1 \\
\text { Y IM }\end{array}$ & \\
\hline & DAC & RFLXE & WALUE OF XYZ \\
\hline & $\mathrm{LAC}$ & FE & \\
\hline & CMA & & \\
\hline & ADD & FL & \\
\hline & $\mathrm{DHC}$ & L. T. & \\
\hline & $\mathrm{J} 1 \mathrm{~s}$ & $T W \cdot L Y$ & \\
\hline & SOR & 1000000 & \\
\hline & Dac & 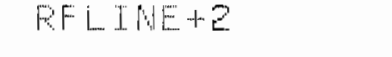 & YSETUO DISFLAY. \\
\hline & LAC & TPACK +e & \\
\hline & 315 & L.TN & \\
\hline & DHC & RH L...'非 $\quad$ SORE & VALUE OF YZ \\
\hline & $\operatorname{LAL}$ & 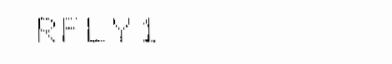 & ' \\
\hline & GA & DE & \\
\hline & ADI & FFLYE & \\
\hline & 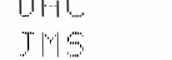 & 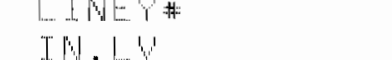 & \\
\hline & ए1ि & H & SETIJP DISPLAY. \\
\hline & $T M F *$ & KFYBD & 。 \\
\hline \multirow[t]{6}{*}{ som } & LHC & ESAJ CHAET & \\
\hline & Danc & DSFTLE & \\
\hline & 1.10 & CEAJ+TRACK 1 & SET TO LREE \\
\hline & DAC & TAGCK $1-1$ & TRACKTHG CROSS \\
\hline & $\mathrm{TMS}$ & SETUF & \\
\hline & THP. & KE & \\
\hline \multirow{5}{*}{ SET } & & & \\
\hline & CUPSOR & INCFEMEMT & \\
\hline & LAC & $\operatorname{Pos} 1$ & \\
\hline & {$[1+\mathrm{C}$} & $P 0 s$ & \\
\hline & LAC & NEO 1 & \\
\hline
\end{tabular}




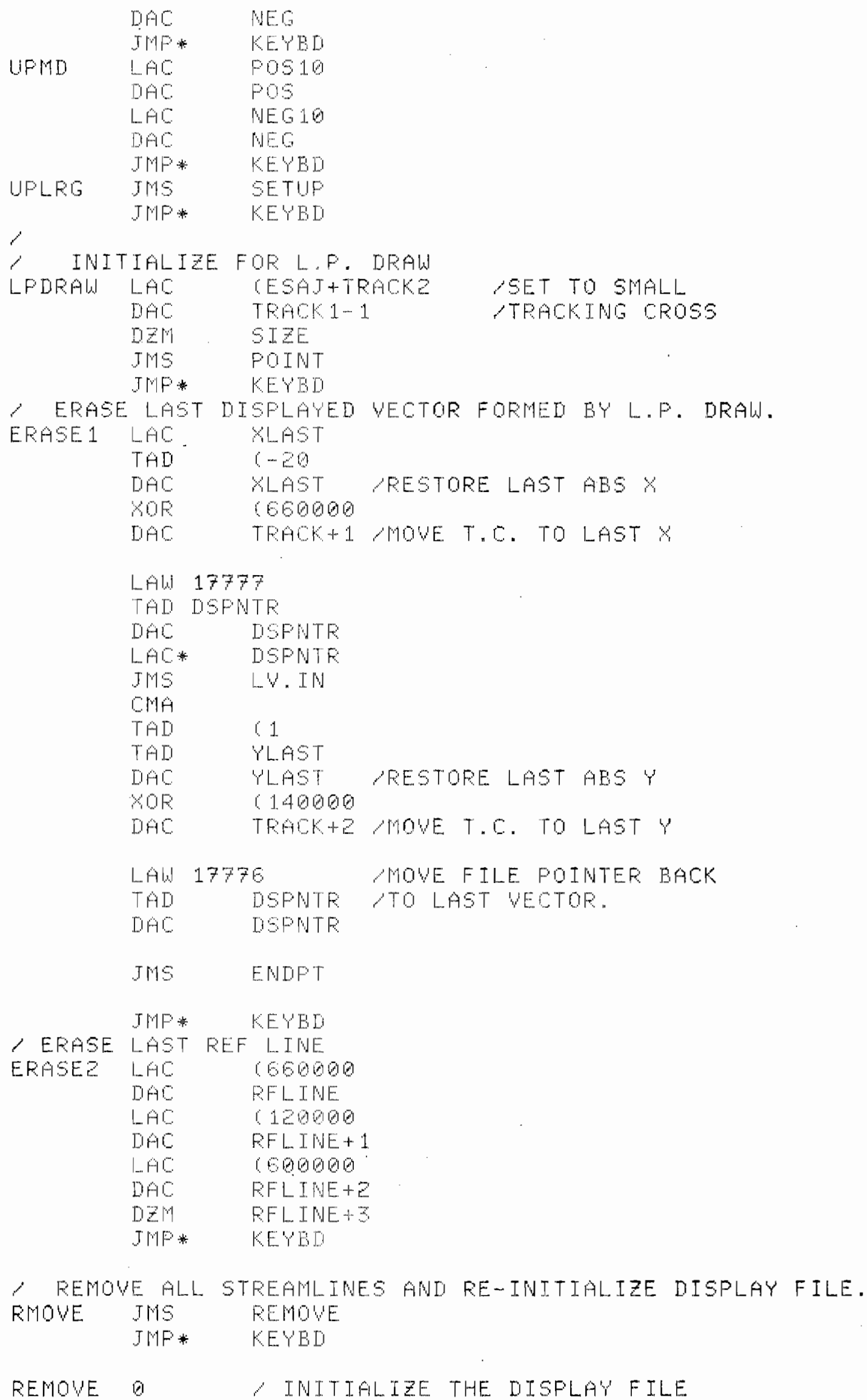




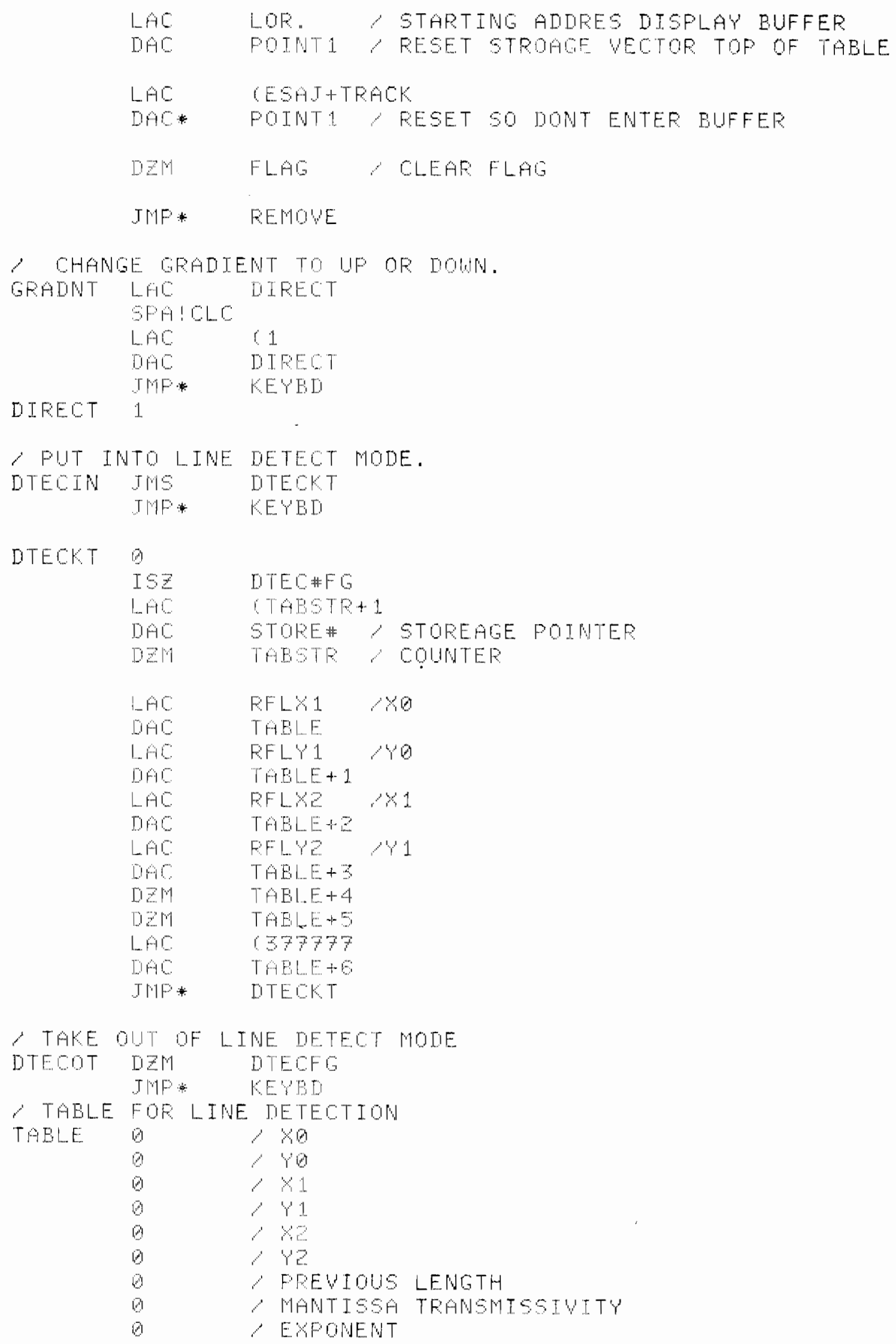




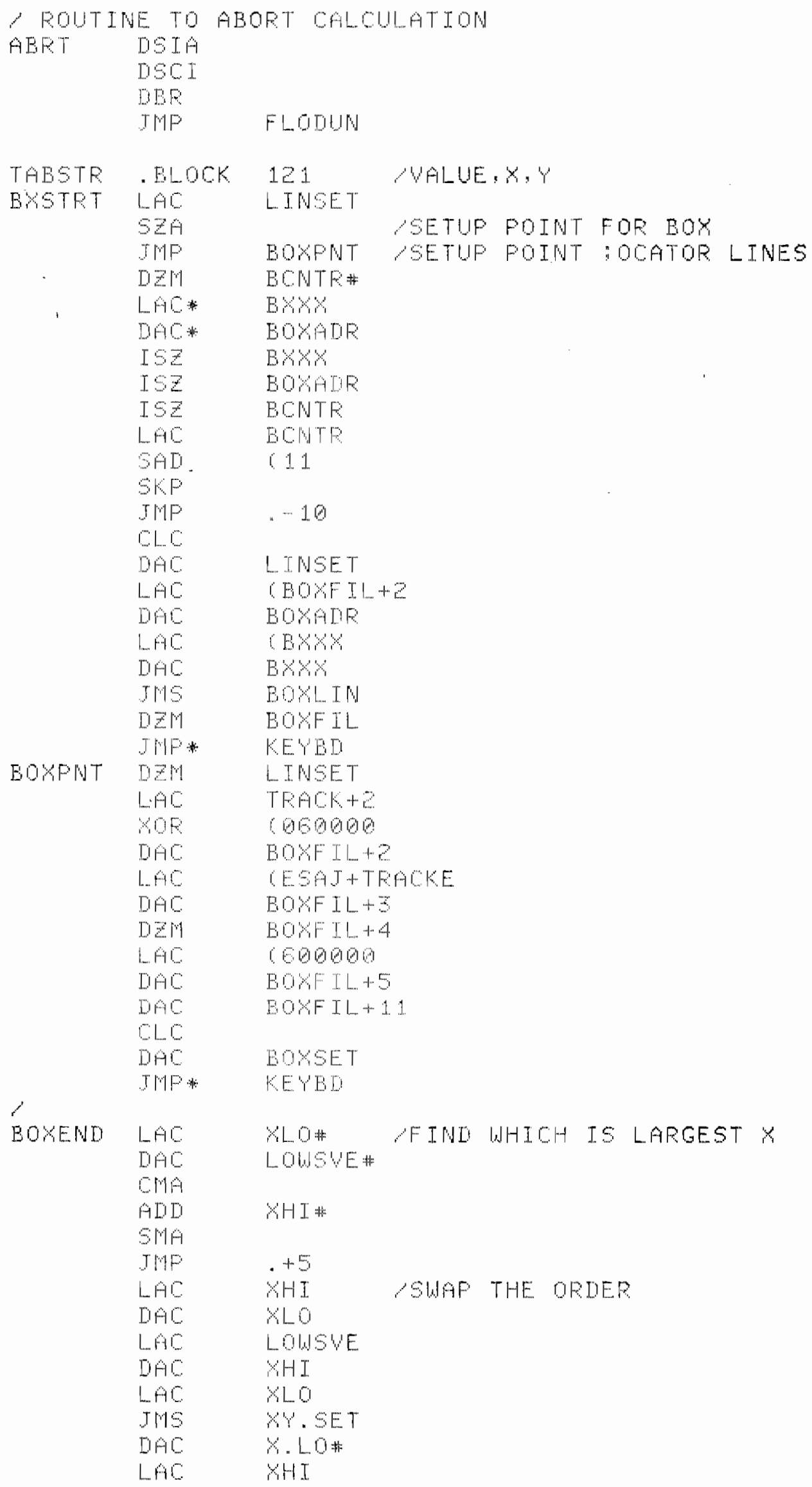




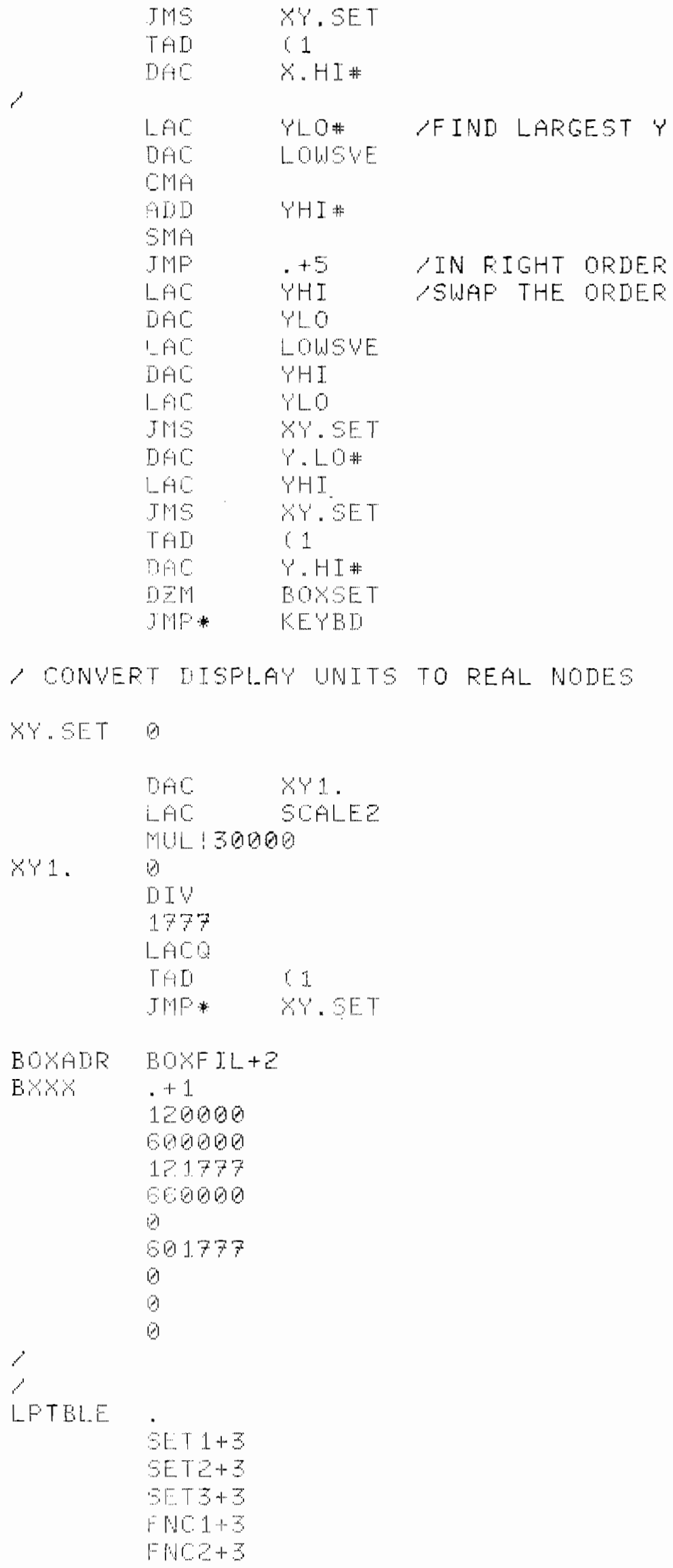




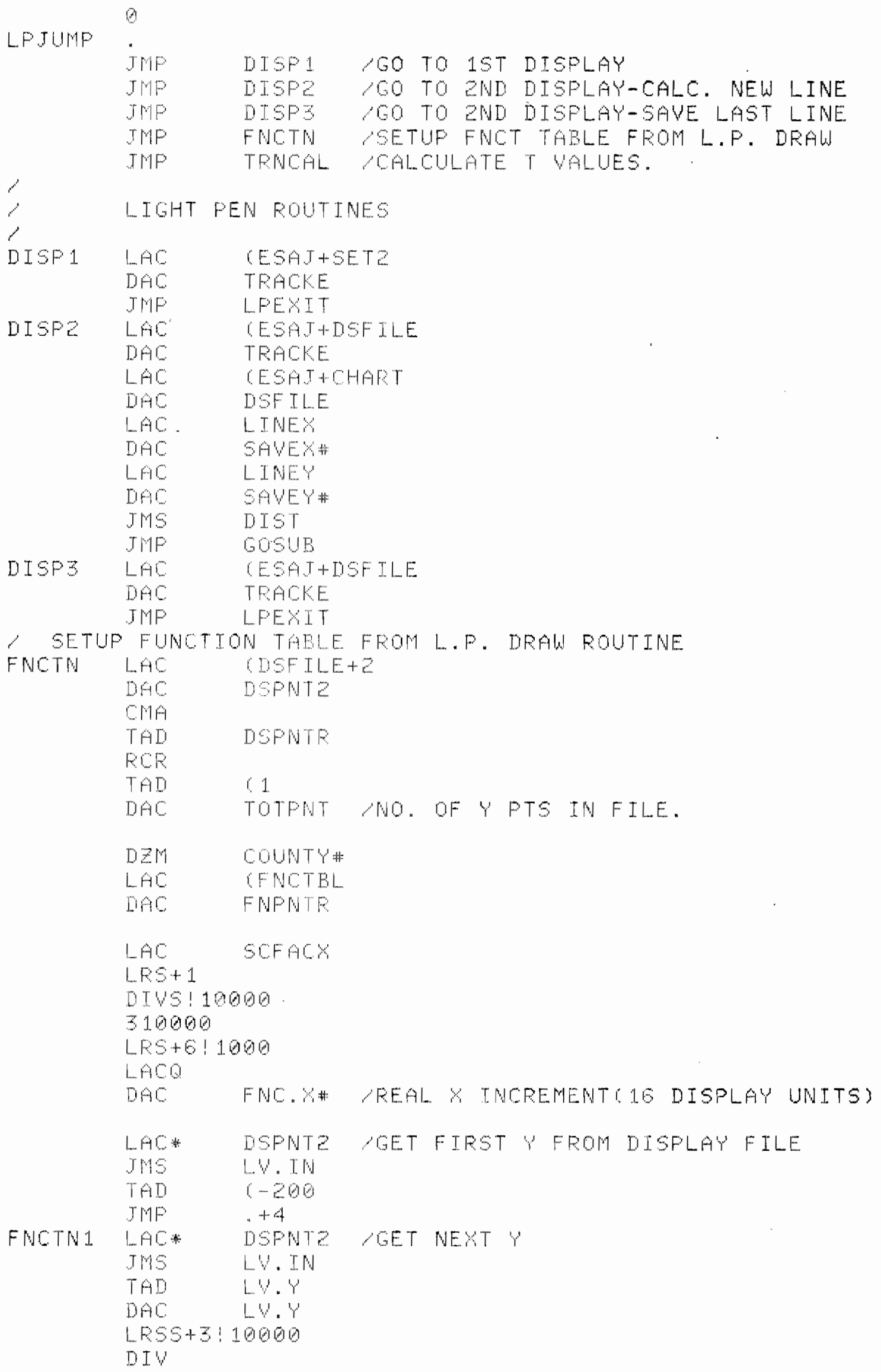




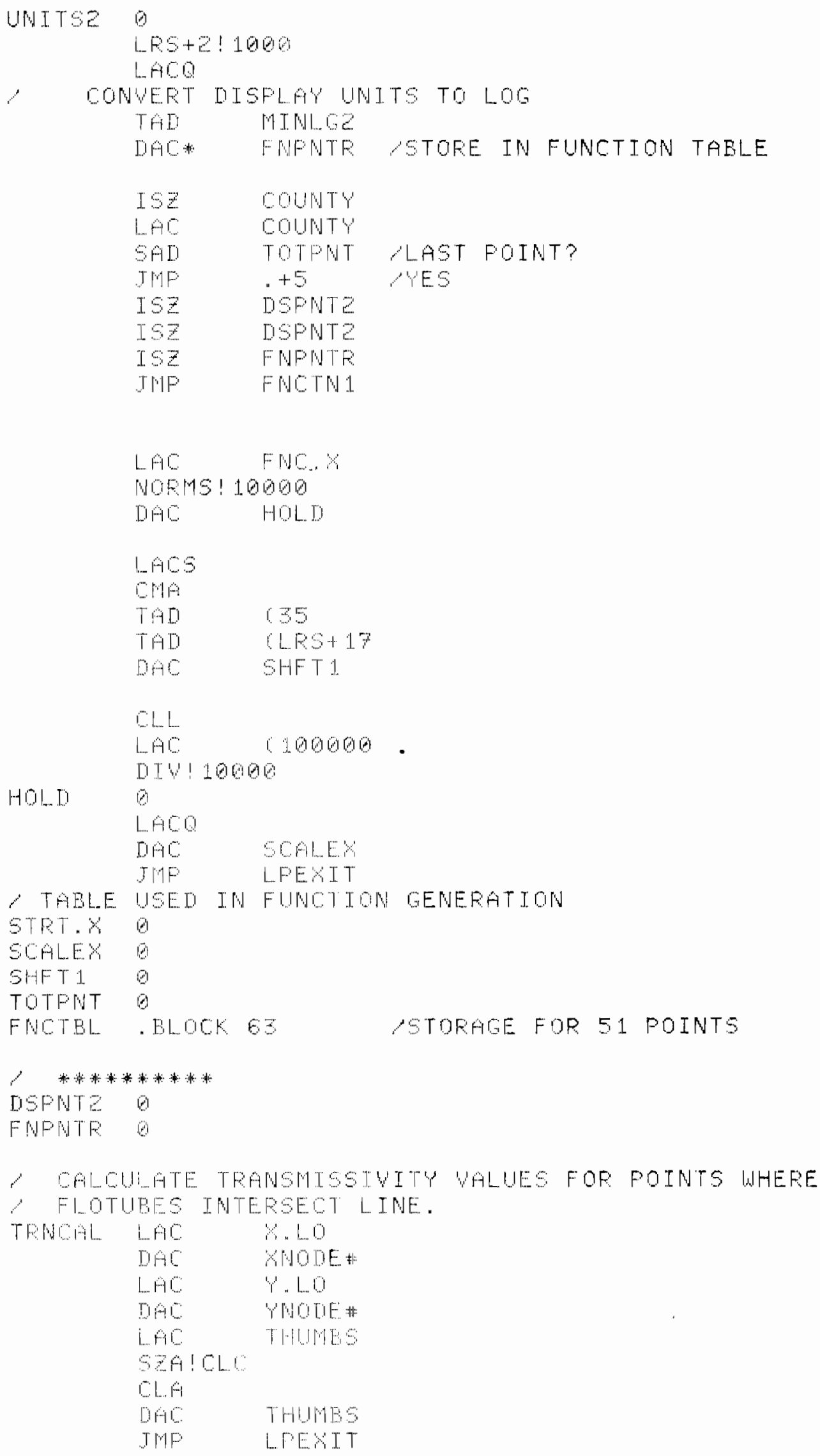




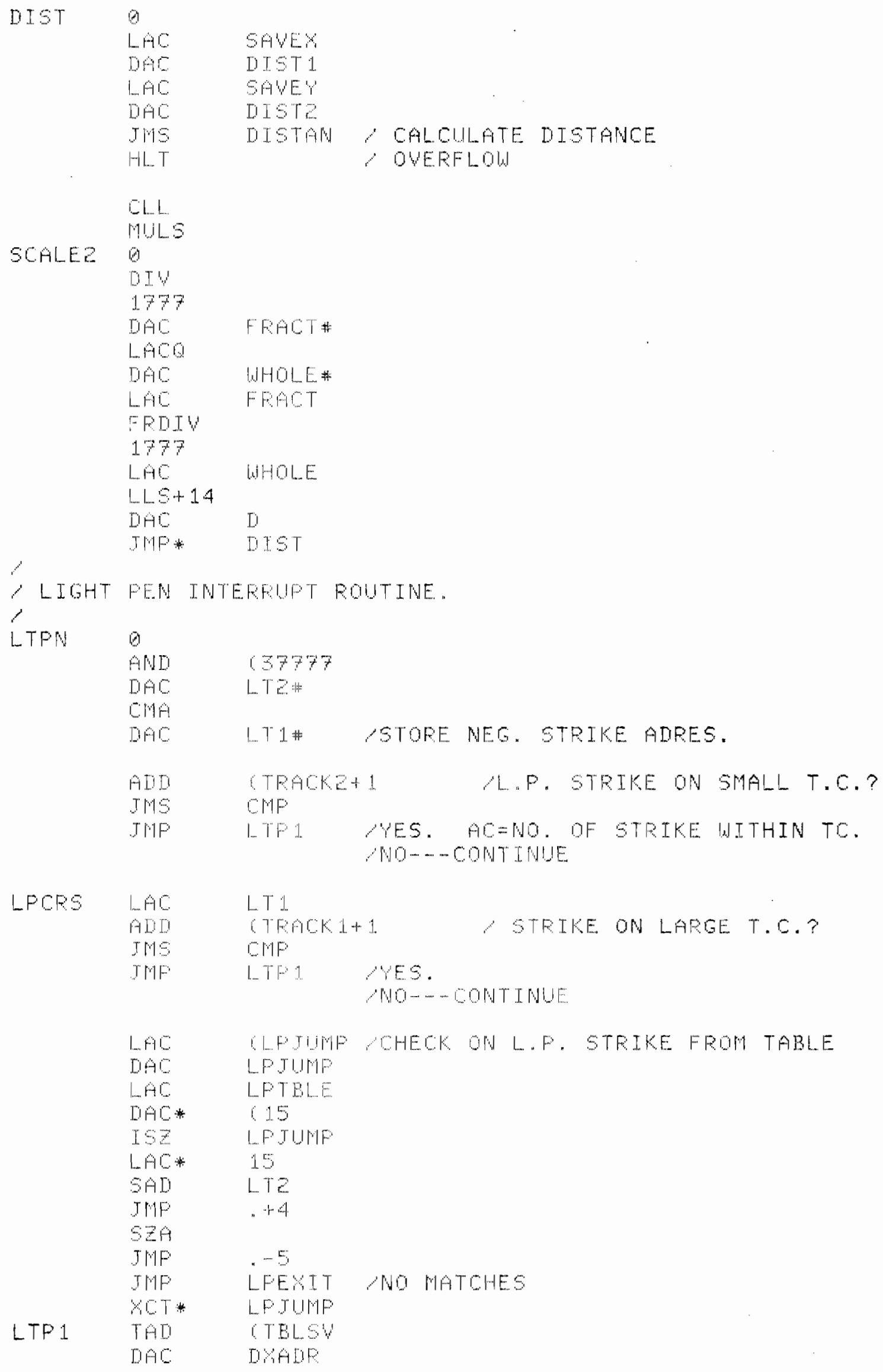




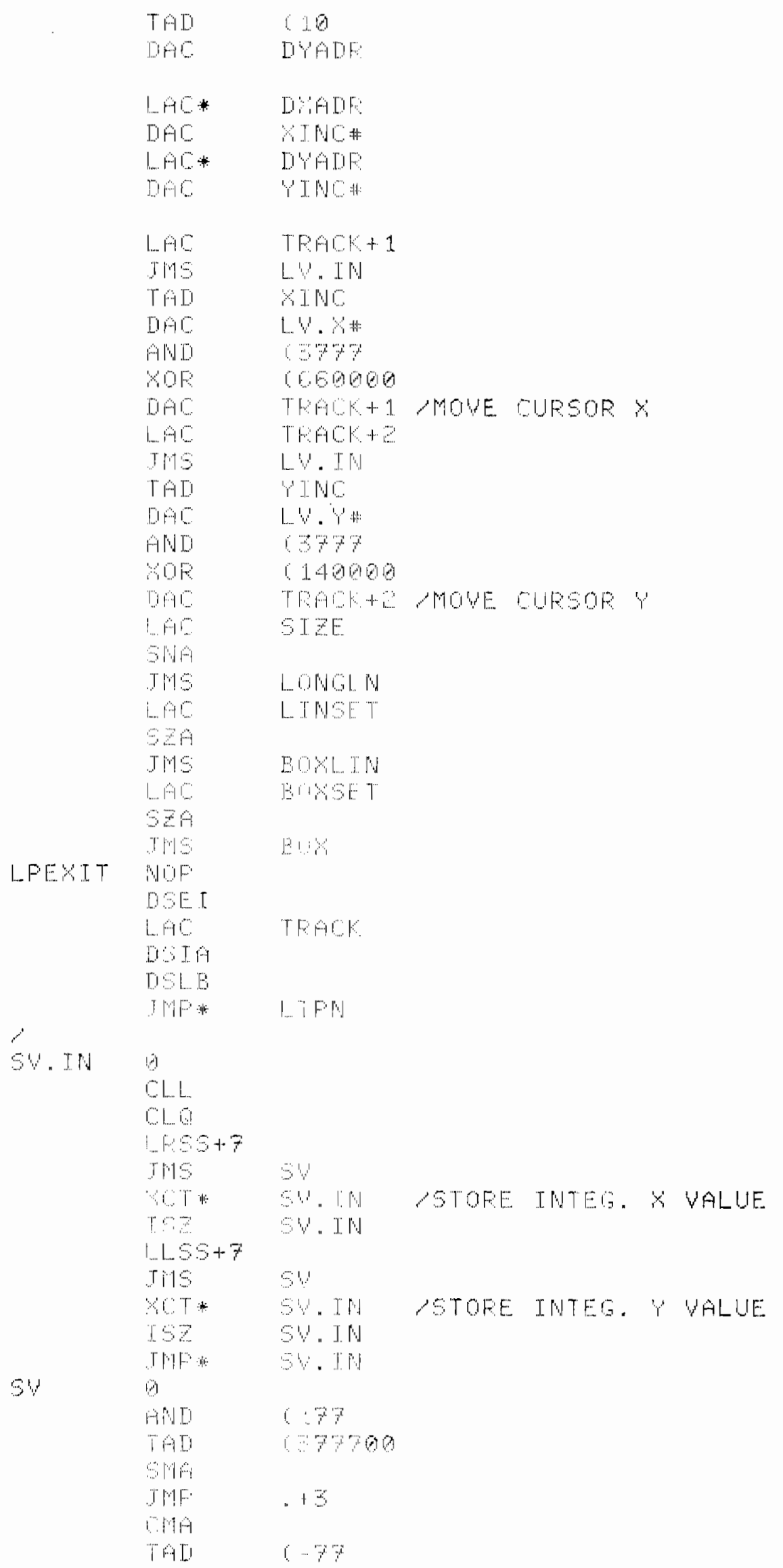




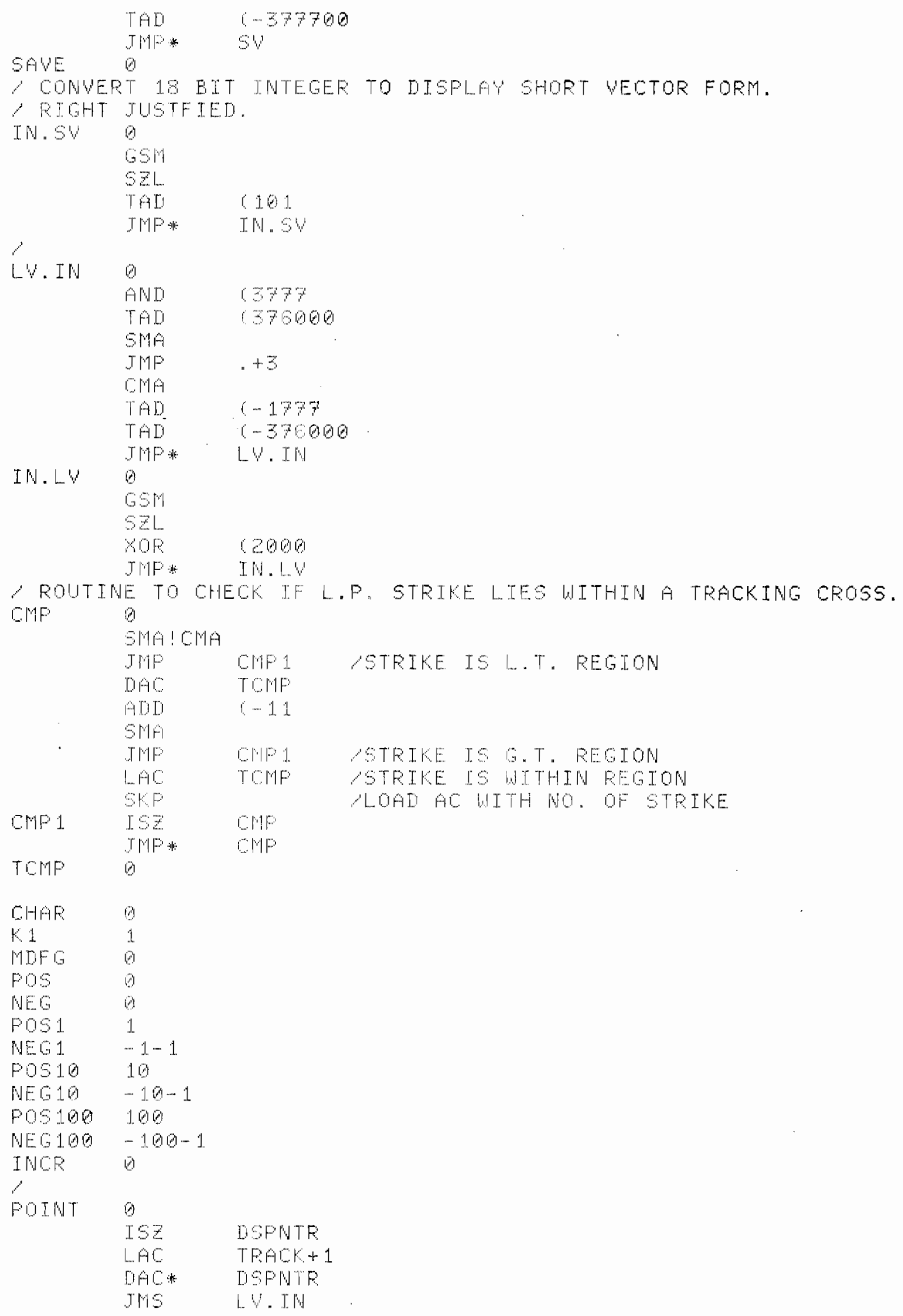




\begin{tabular}{|c|c|}
\hline DAC & Кㄴ..f9T \\
\hline ISZ & DSFITR \\
\hline L...... & TEACK+2 \\
\hline DAC* & DSPUTR \\
\hline$\pi 116$ & 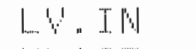 \\
\hline DAC & YLAST"\# \\
\hline IMS & EMPT \\
\hline DEY & DSFTLE \\
\hline
\end{tabular}

LONGLN O

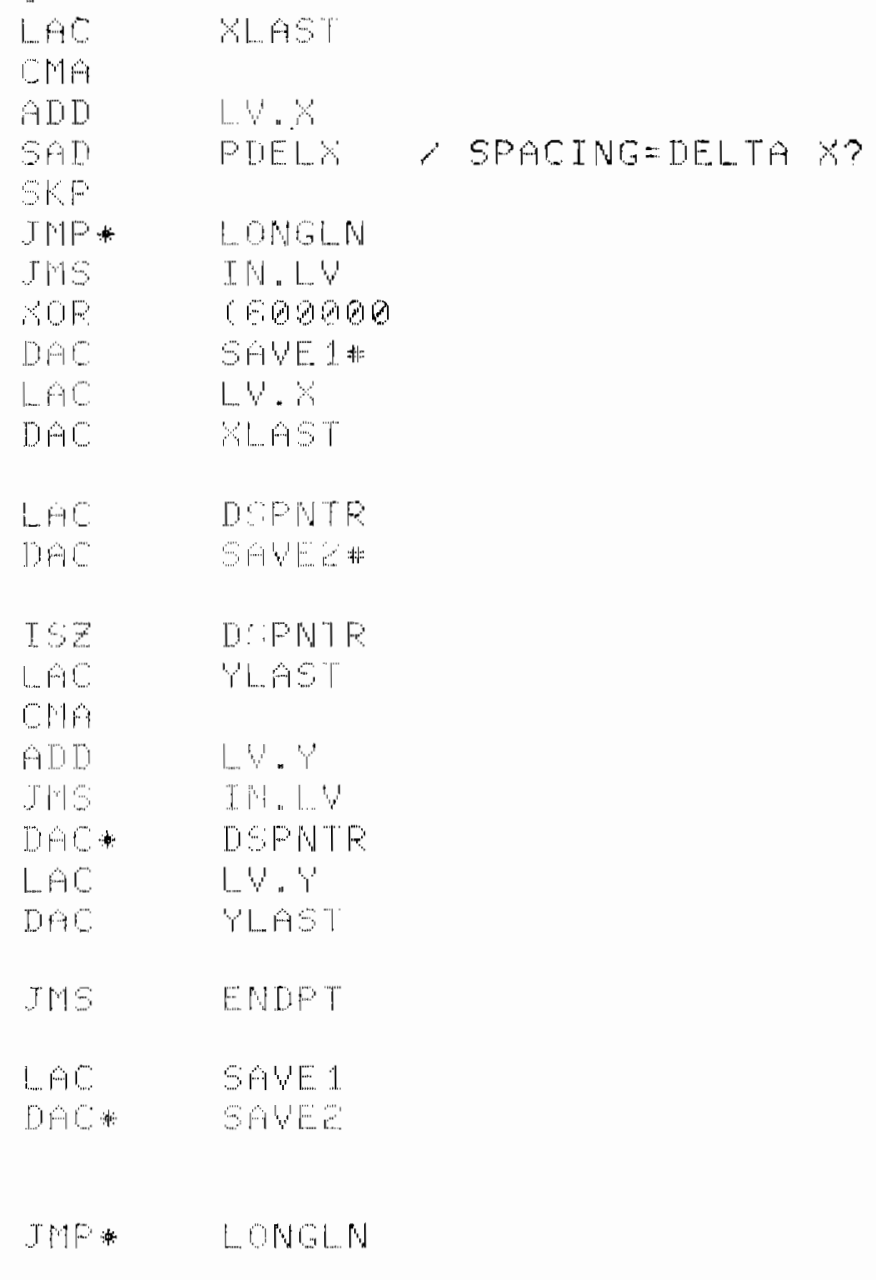

ENLPT

$\begin{array}{ll}\text { ISE } & \text { TSPNTR } \\ \text { LAC } & \text { SEAT DOHART } \\ \text { DACH } & \text { DSPNR } \\ \text { JWPA } & \text { ENDPT }\end{array}$

PDELK 29

ROUTINE TO OUTPUT VERTCAL \& HORIZONTAL LINES

'THFU THE T.C. (FOR TRANSMISSIUTTES)

BOYLIL 


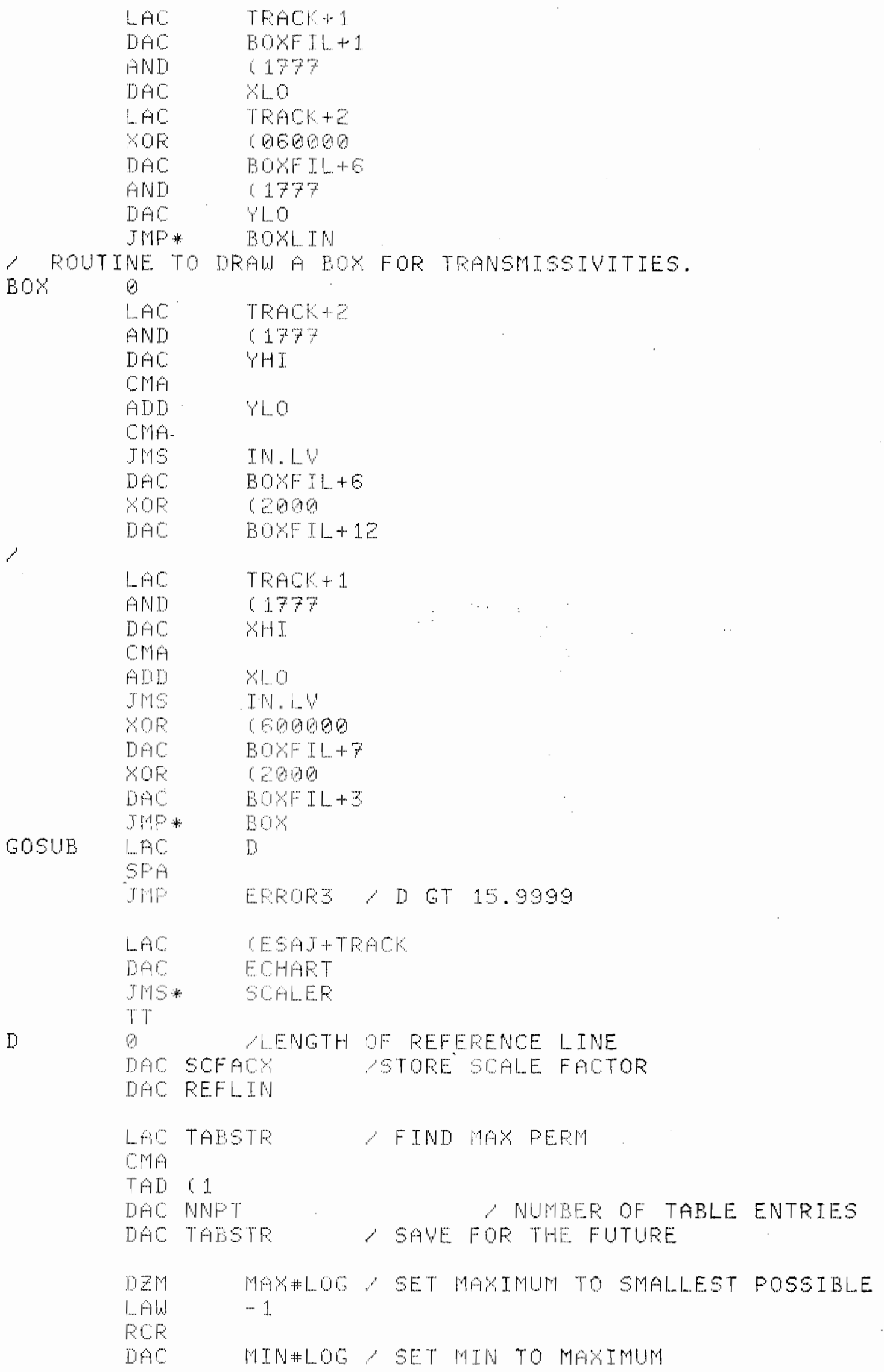


LAC THASTE+3 TAELE FORMAT, THIS IS FIRST LOG

MAKLOG DAC ALRE ZRETEIUAL POINTER

JMS* FPSL. FORM LOG

$400000+A D R E$

DAC* ATRE STORE

JMS* CHP. FIMD MAX AND MIN

LAC MAKLOG

NOP $>$

DAE MANLOE $=$

JMS* CMP.

LAC MTLOS

$\mathrm{TWF} \quad-3+3$

WOF

DAL MIMLOE

ISZ ADEZ $Y$ ZERO ESFONENT

DZW

LAC ADEE

TAI

ISZ UHFT

TMP MAKLOG

LAC GY1 NOL FOF THE SCALE

DAE ADEE THIS IS FOF SGALE PRINTING

$\angle A O \quad \therefore 72$

DAC AESFT THIS IS FOF GRAPHING

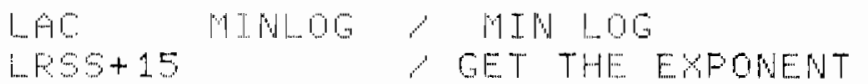

WAC UALUE\# SAVE THE EXFONENT MAGNITUDE

314

TAD 1

WAC SFREAD 2 S COMPLIMENT FOWER OF TEM

$1 . .68+15 ! \mathrm{CLO}$

DAC MU⿴囗十

CMP

TAD 1

DAC MISLEZ

LAC MAKLOG MAX LOG

L..PSS+15

TAD $\quad$ PREAD

TAD

TAE SPEEAD DELTA POLER OF TEN

CLL WOW FLND THE NUMEER OF DISFLA'Y UNITS

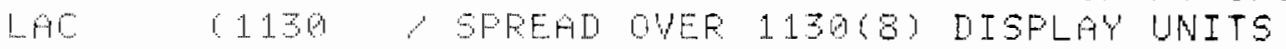

SFREAD

I. DI Y

LACO

DAC

DAC

UNIS $\quad$ WUMBER OF DISPLAY UNITS PER DECADE

CNA UNT TSE

$\checkmark$ FOR FIRST TIME THEOUGH 


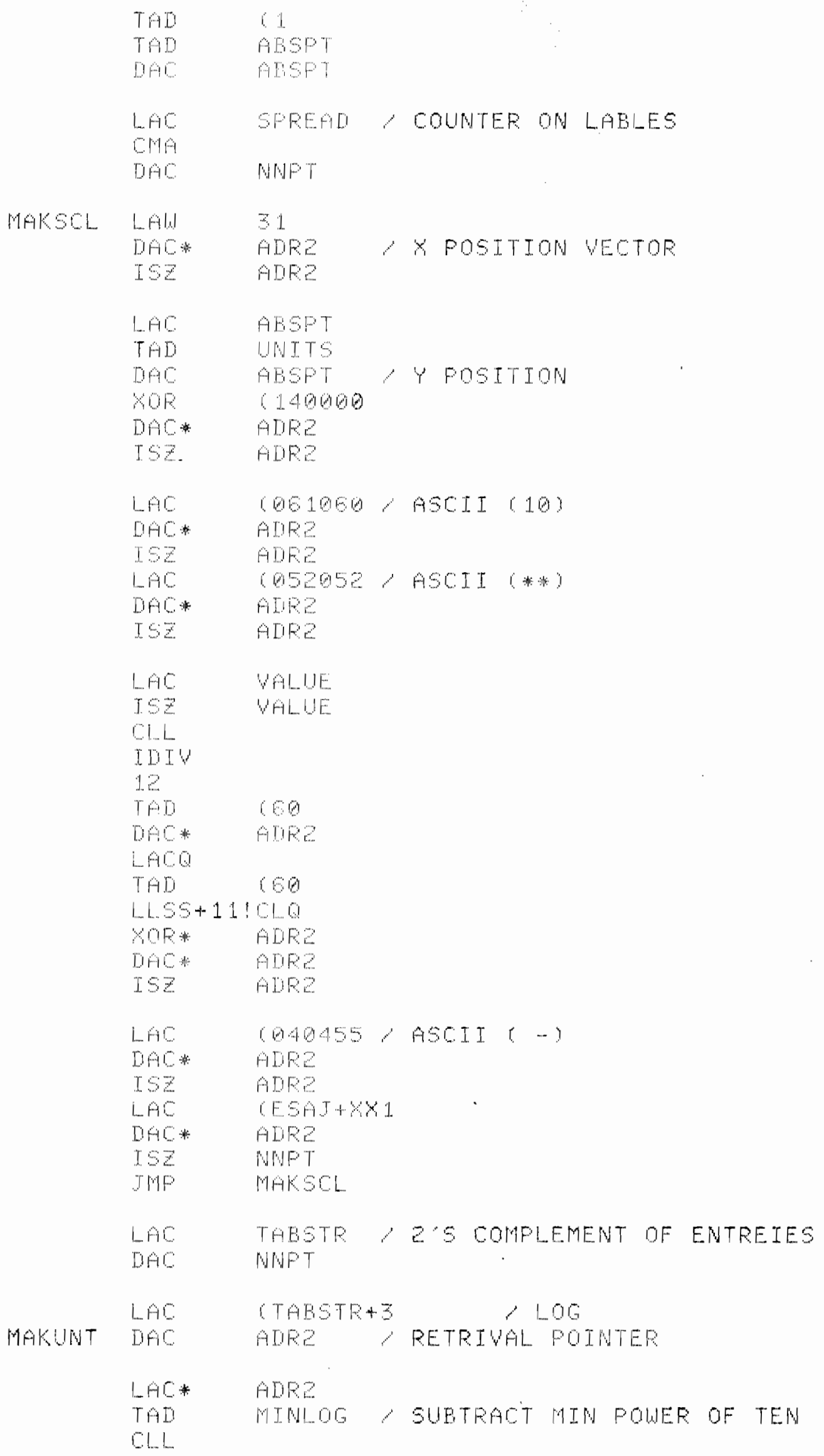




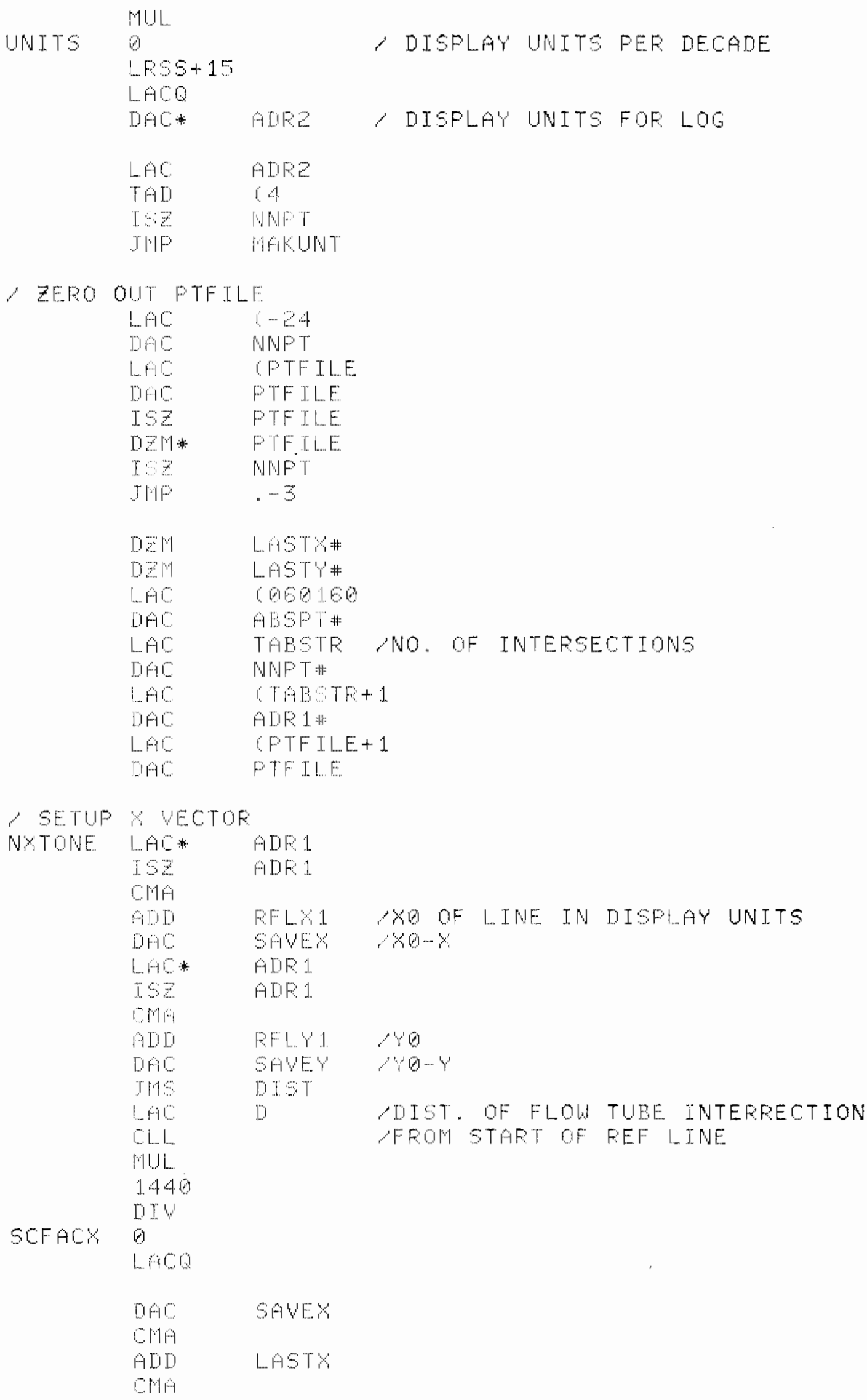




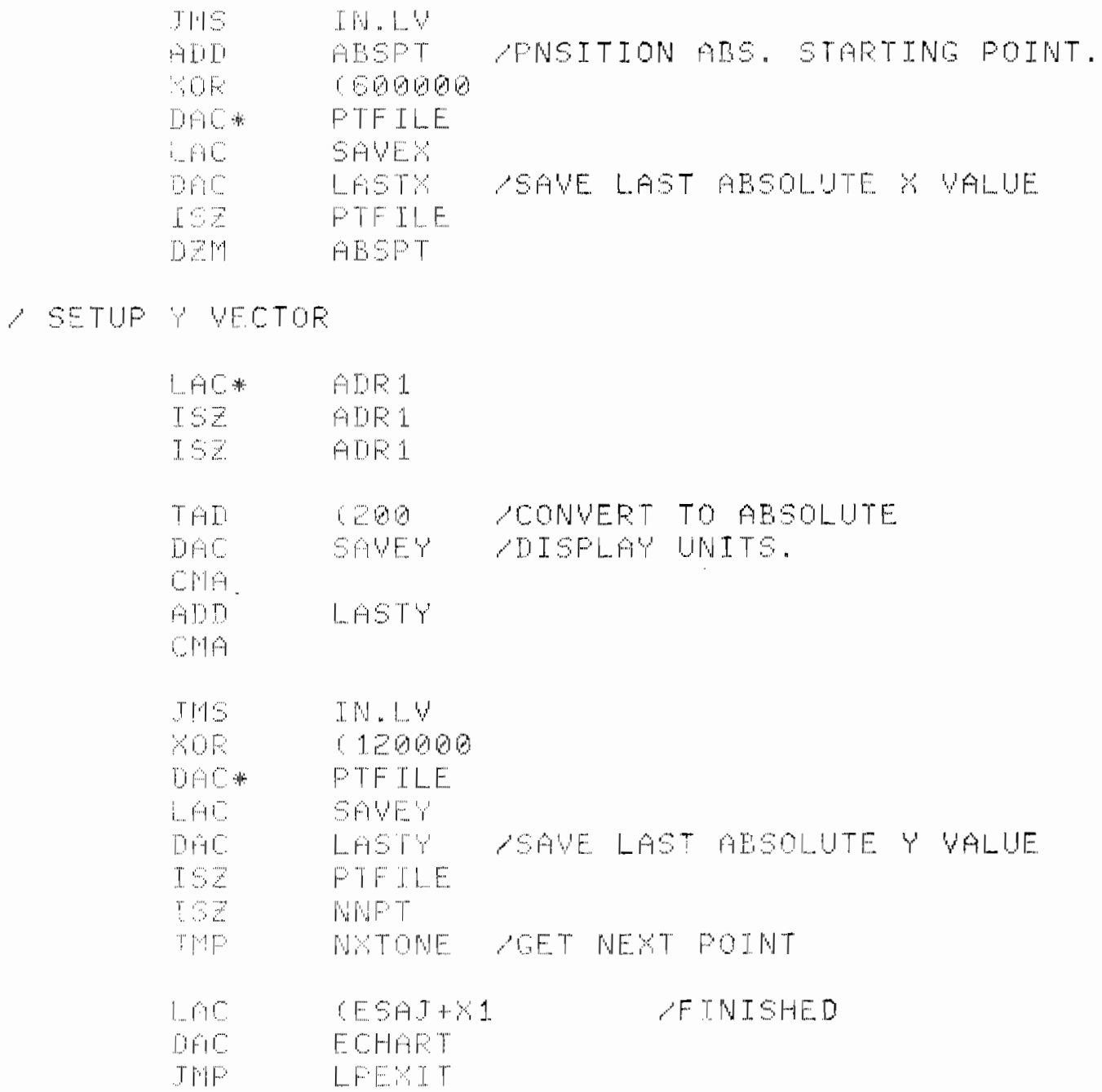




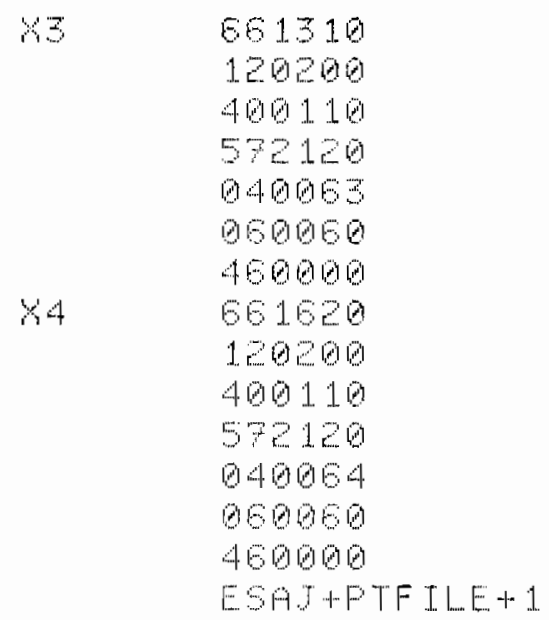

$\checkmark$ FILE TO STORRE FLOLTURE INTERSECTION POINTS

$\therefore$ WITH INTERSECTTWG LINES

PTFILE.

- BLOCK 50

ESAT+TRACK

?

ERRORS

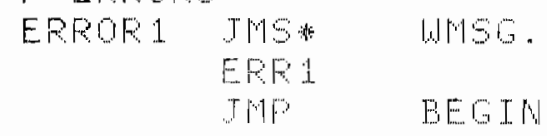

ERPORE JMS* WMO.

EPRE

EREOR3 JM W MS6.

EFES PEMOHE

$\begin{array}{ll}\text { JWS } & \text { PEMOWE } \\ \text { JMF } & \text { EFPE? }\end{array}$

EREORS JMS* WMS.

EFES

EXIT

BUFFERS

LEUF $\quad$ BLOCK $2 Q$

THTS IS THE BUFFER THAT IS COMMON TO ALL PROGRAMS IN THE ITERATIVE

SEOUENOE

IT TS OHE DISO SETTOR LONG (112 WORDS?

IT IS OTIDED URTO 3 SETTONS

1. DTSE ADDFESS OF THE FROGRAMS

$\therefore \quad$ E. MPS

3. COMMOW DATA

DKMTFL 9 DISO ADDEESS OF MONTOR

DKSTFM DUSO ADDEESS OF SPREAM-LIME

DKFLOW Q DISO ADDEESS OF FI.OW-TUBE ISTEADY-STATE)

DKF TR O DLSE ADOEESS OF FLOW TUEE (TRANSIENT)

DKTEYL O $O$ DISC ADDRESS OF TRAVEL-TIME (TRAMSMISCIUITY)

DKTEUK O DISC ATDRESS OF TRAUEL TIME (CONSTANT FERMIABILITY)

DKINTE Q DISO ADDRESS OF THTEPF

DKCOMB O DTSO ADDEES OF MODIFY MATRIX 


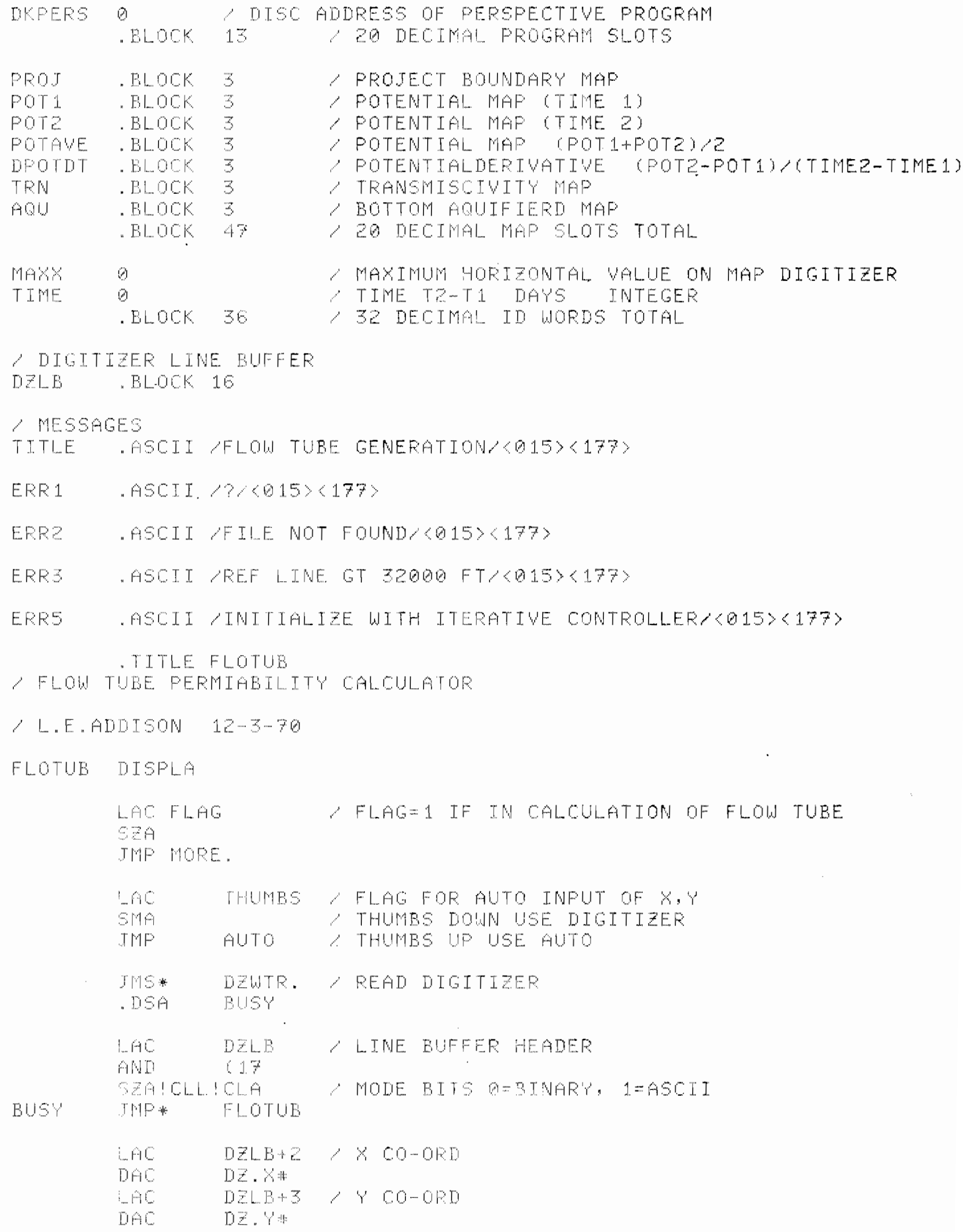




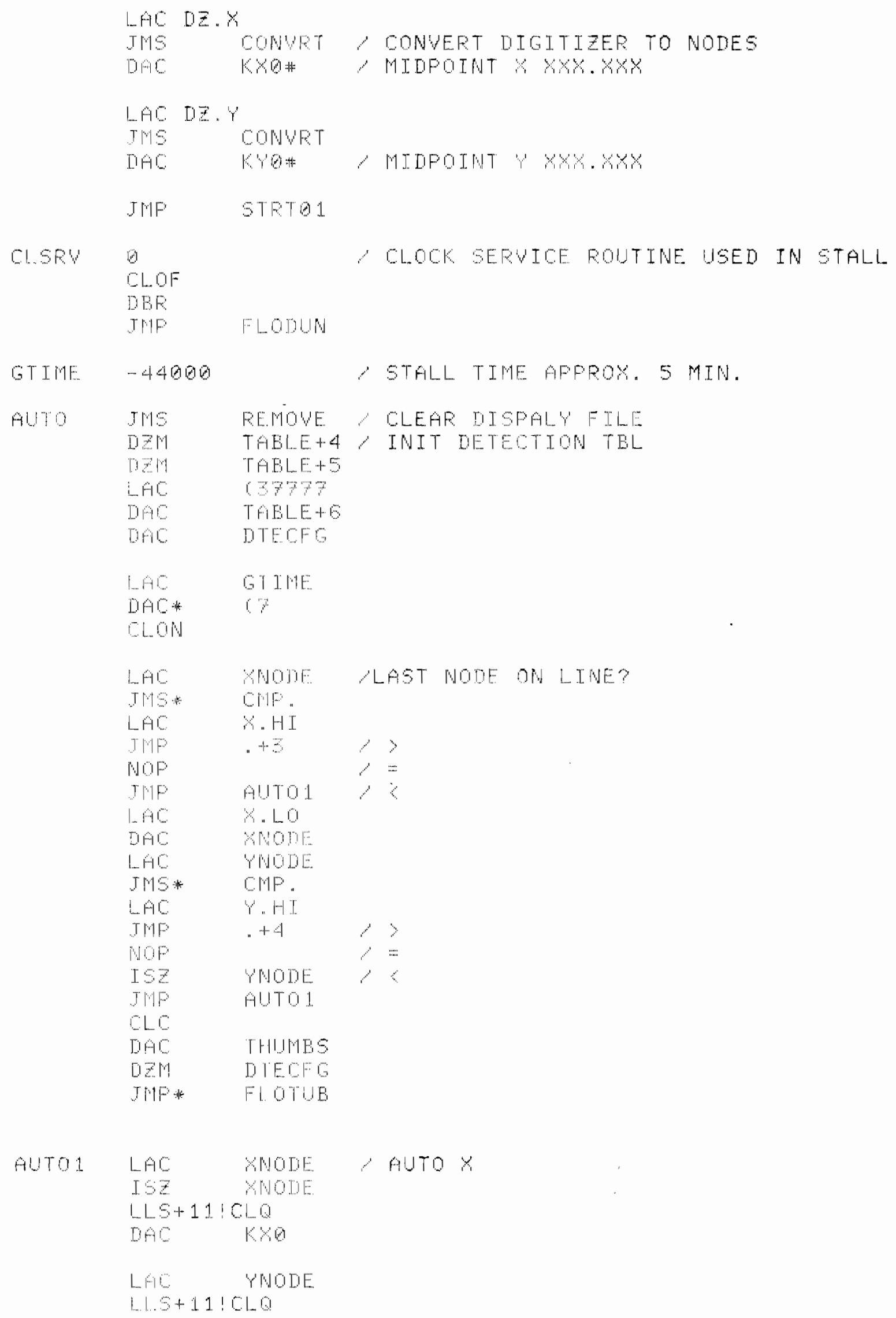




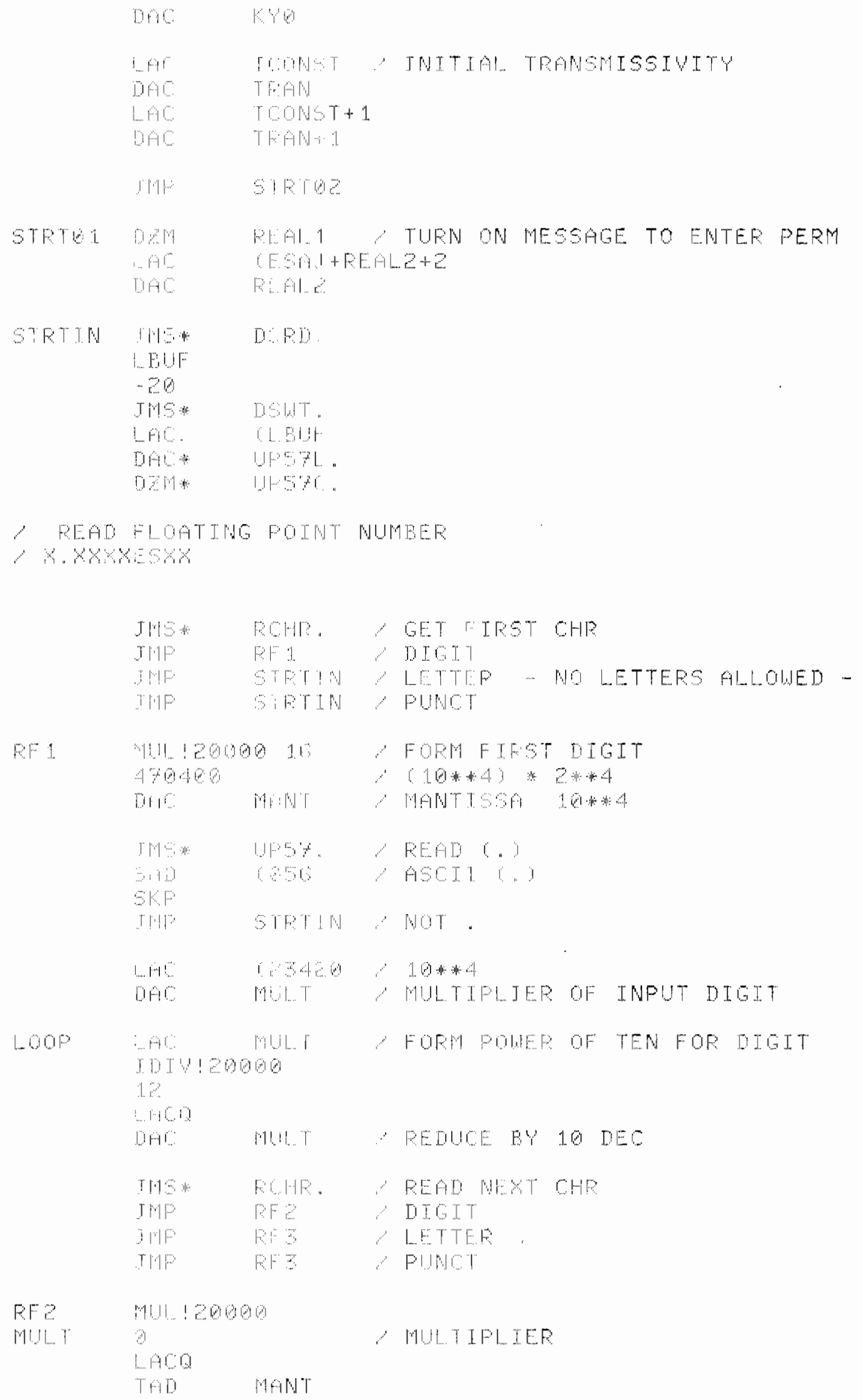




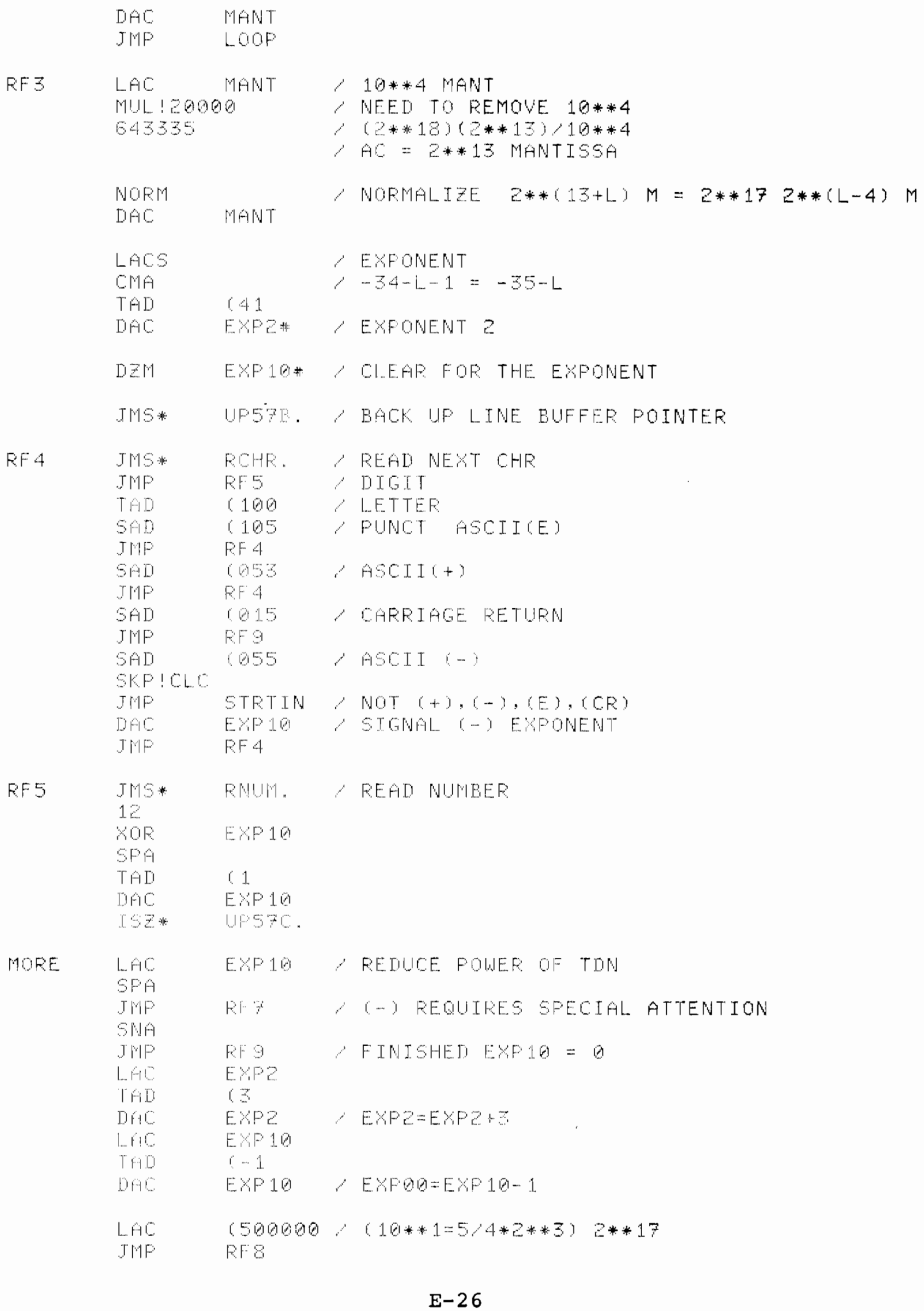




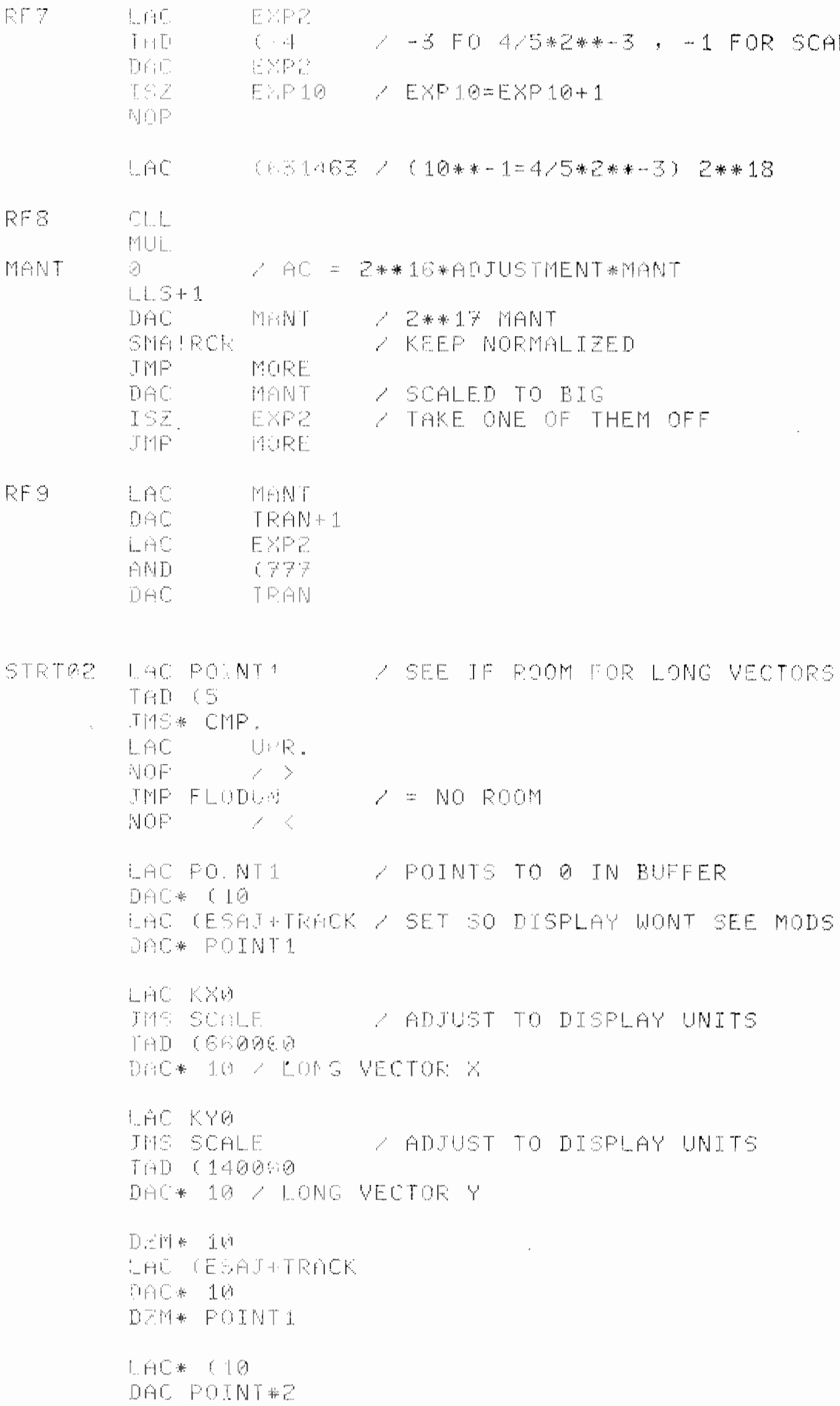




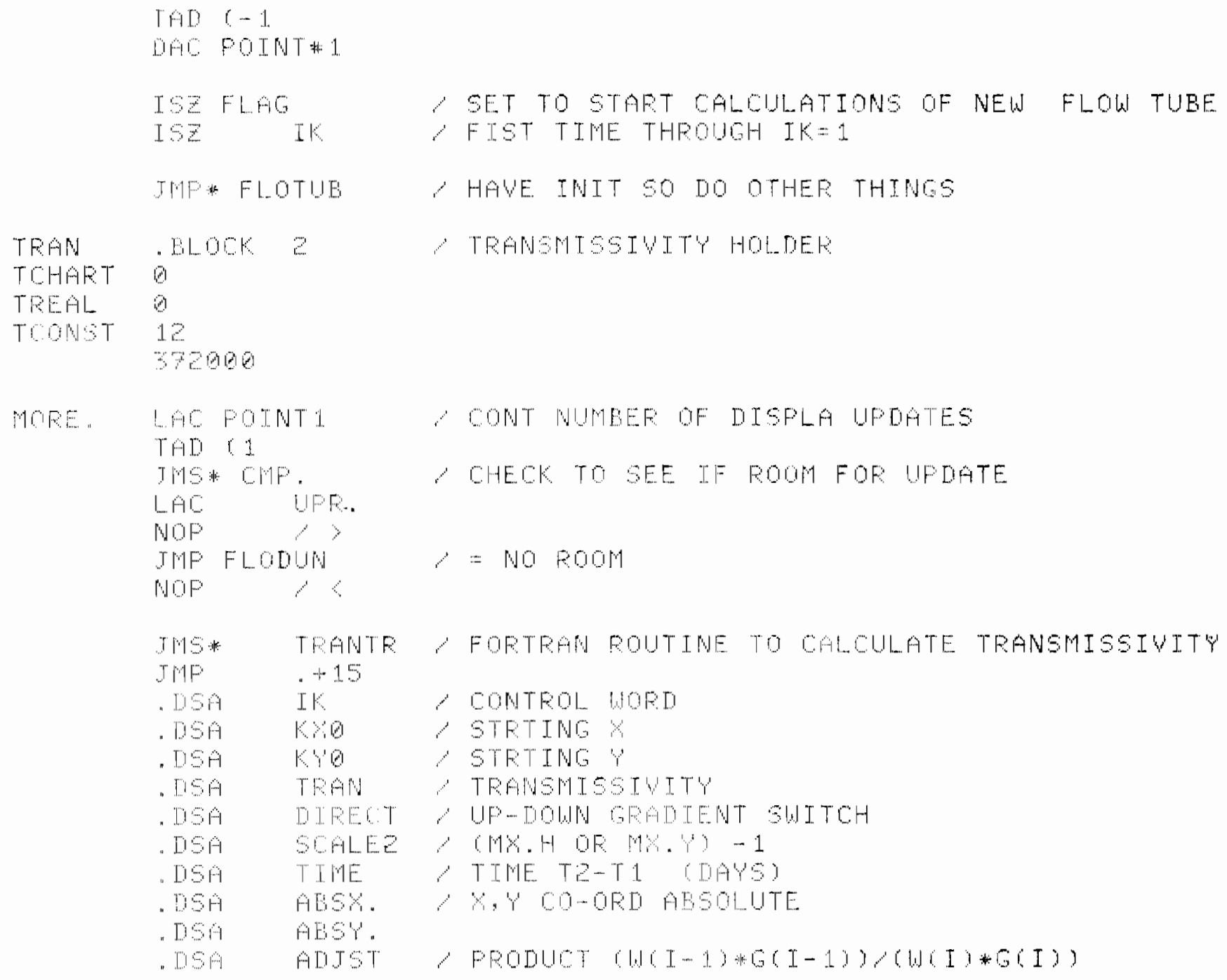

\begin{tabular}{|c|c|c|}
\hline \multirow{2}{*}{$\begin{array}{l}\angle \mathrm{AC} \\
\mathrm{FH} \\
\mathrm{MHF}\end{array}$} & \multicolumn{2}{|l|}{ THUMBS } \\
\hline & YECTS & \\
\hline $1 A \%$ & ABSK. & \\
\hline TW & CMP. & \\
\hline LAए: & XLO & \\
\hline JWF & +3 & \\
\hline$S K F$ & & $=$ \\
\hline $\mathrm{THF}$ & FLOSUR & $<<$ \\
\hline TW & CMF. & \\
\hline LAC & $8+1 \mathrm{~T}$ & \\
\hline$T M F$ & 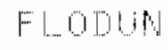 & 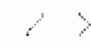 \\
\hline $5 \%$ & $\cdot+E$ & 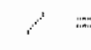 \\
\hline WF & & $<$ \\
\hline $\operatorname{Lan}$ & $\mathrm{BBSY}$. & \\
\hline$J W$ & EF. & \\
\hline LAC & $y<0$ & \\
\hline &.+3 & $y$ \\
\hline
\end{tabular}




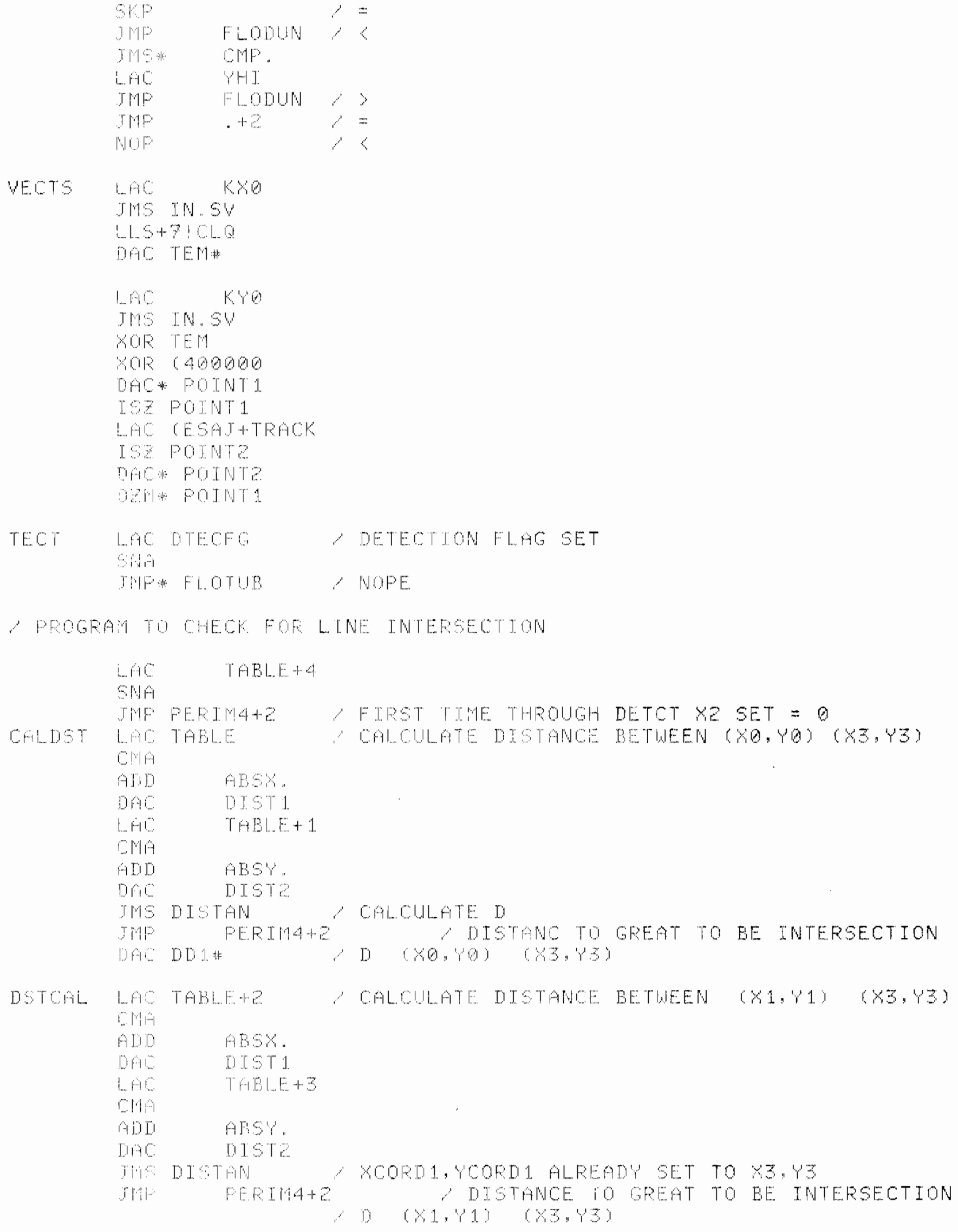




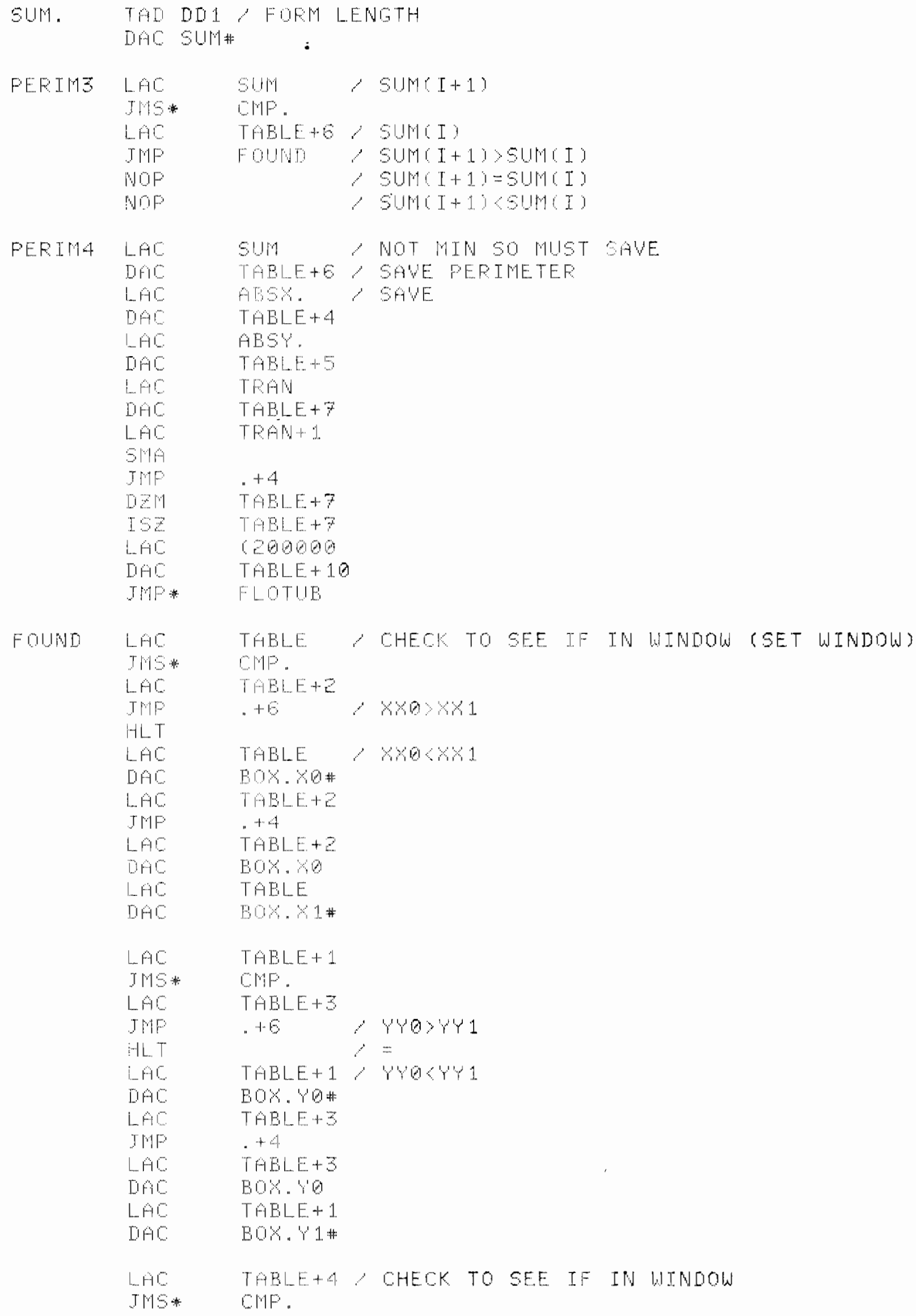




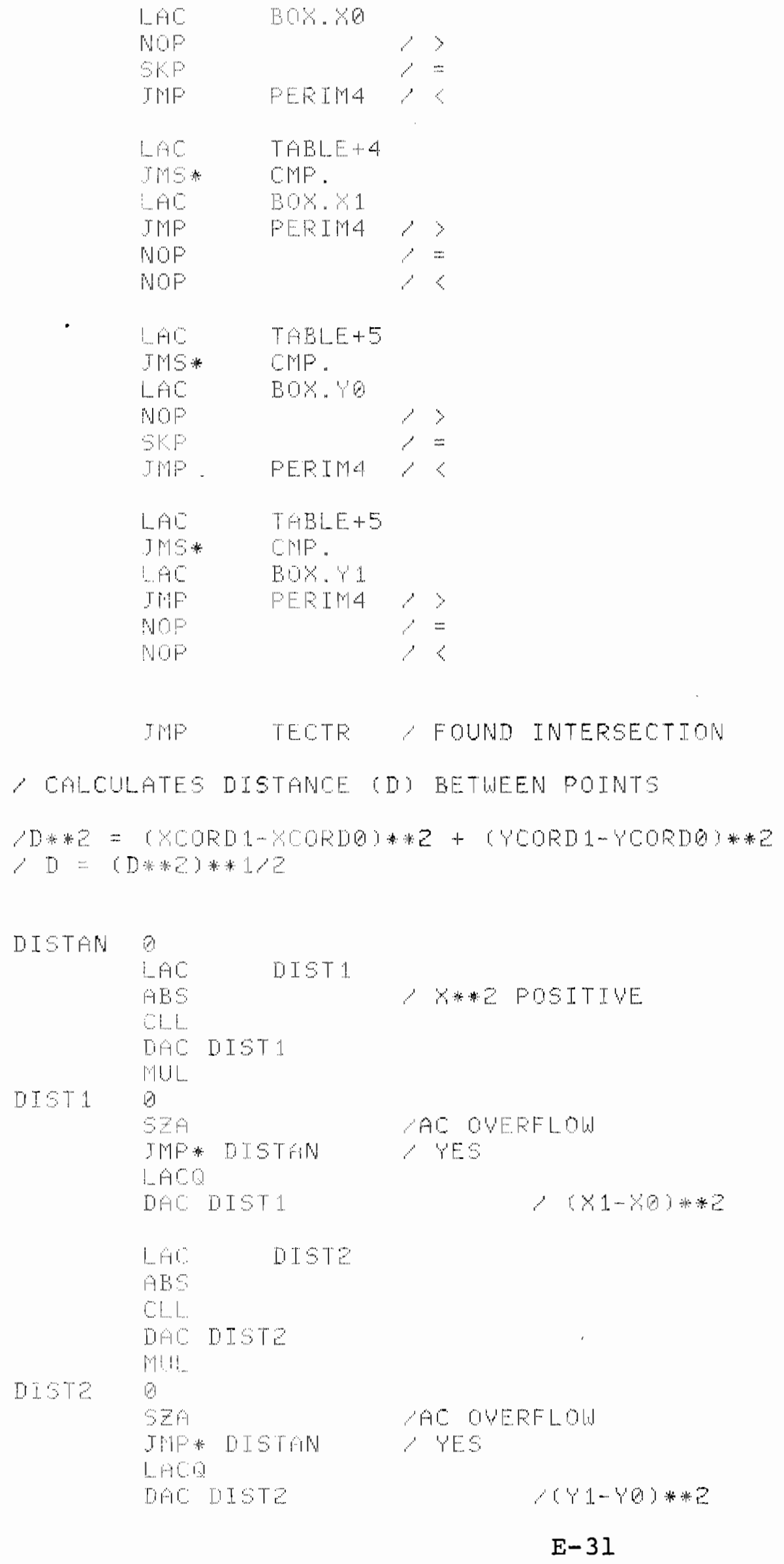




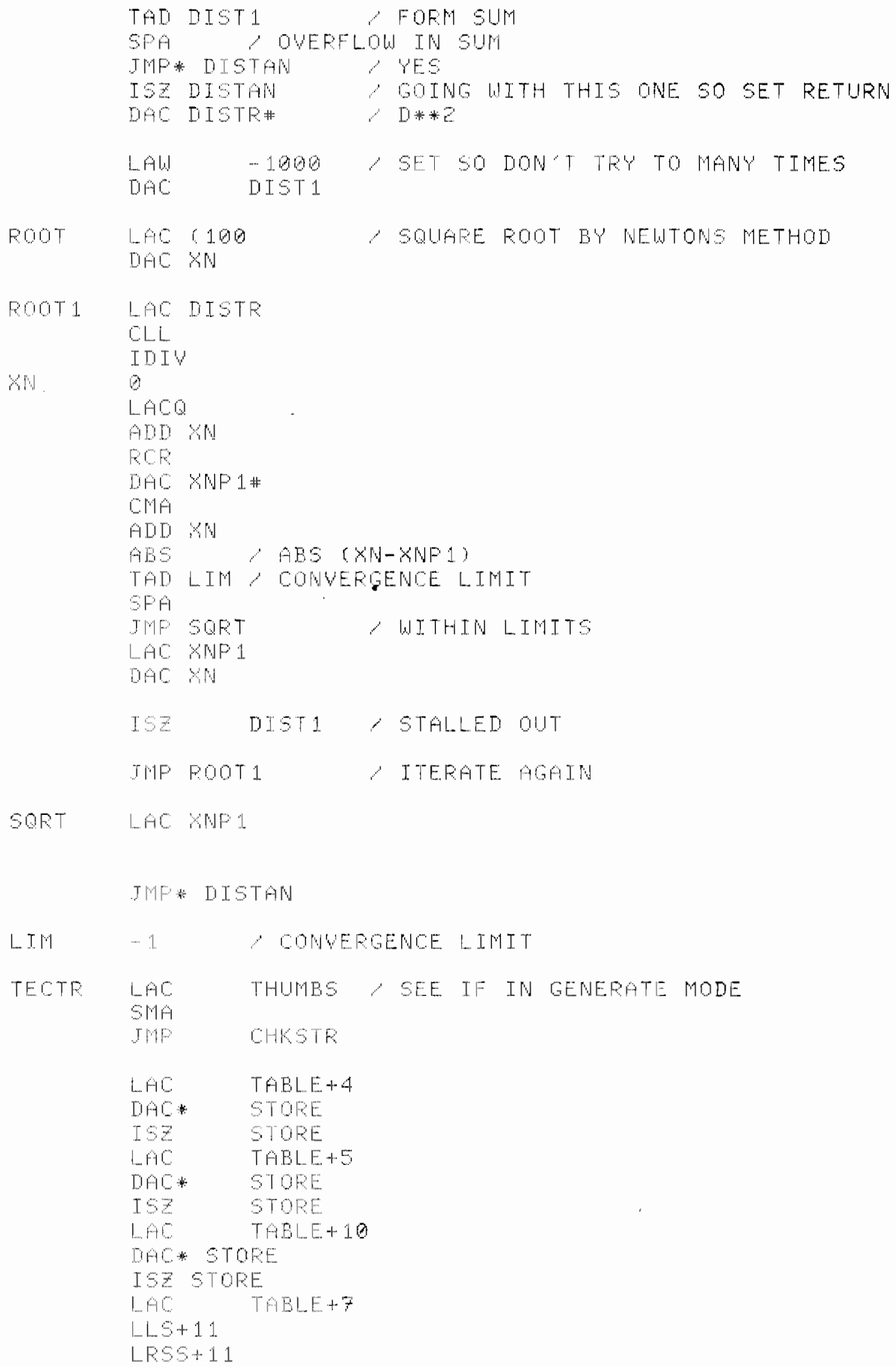




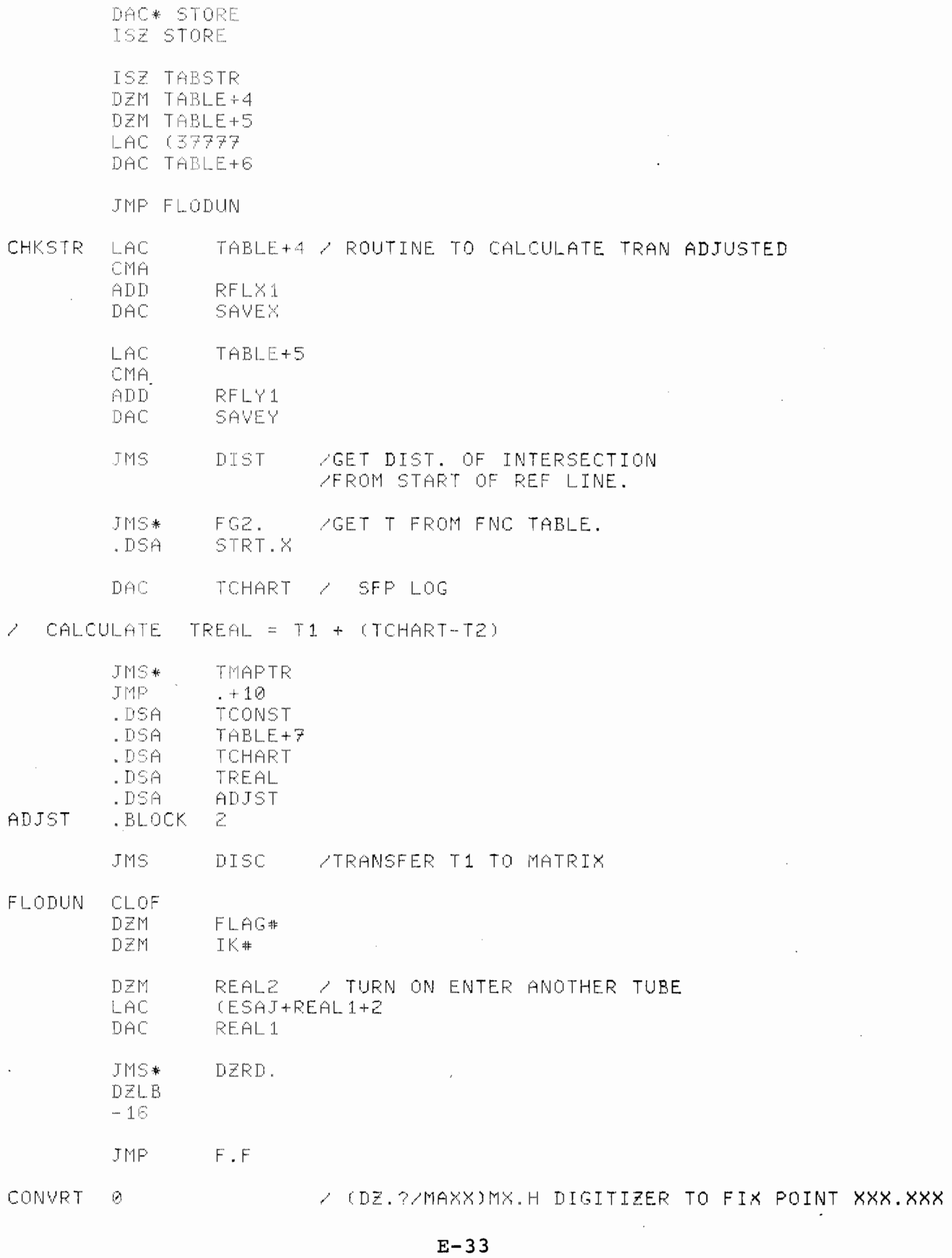




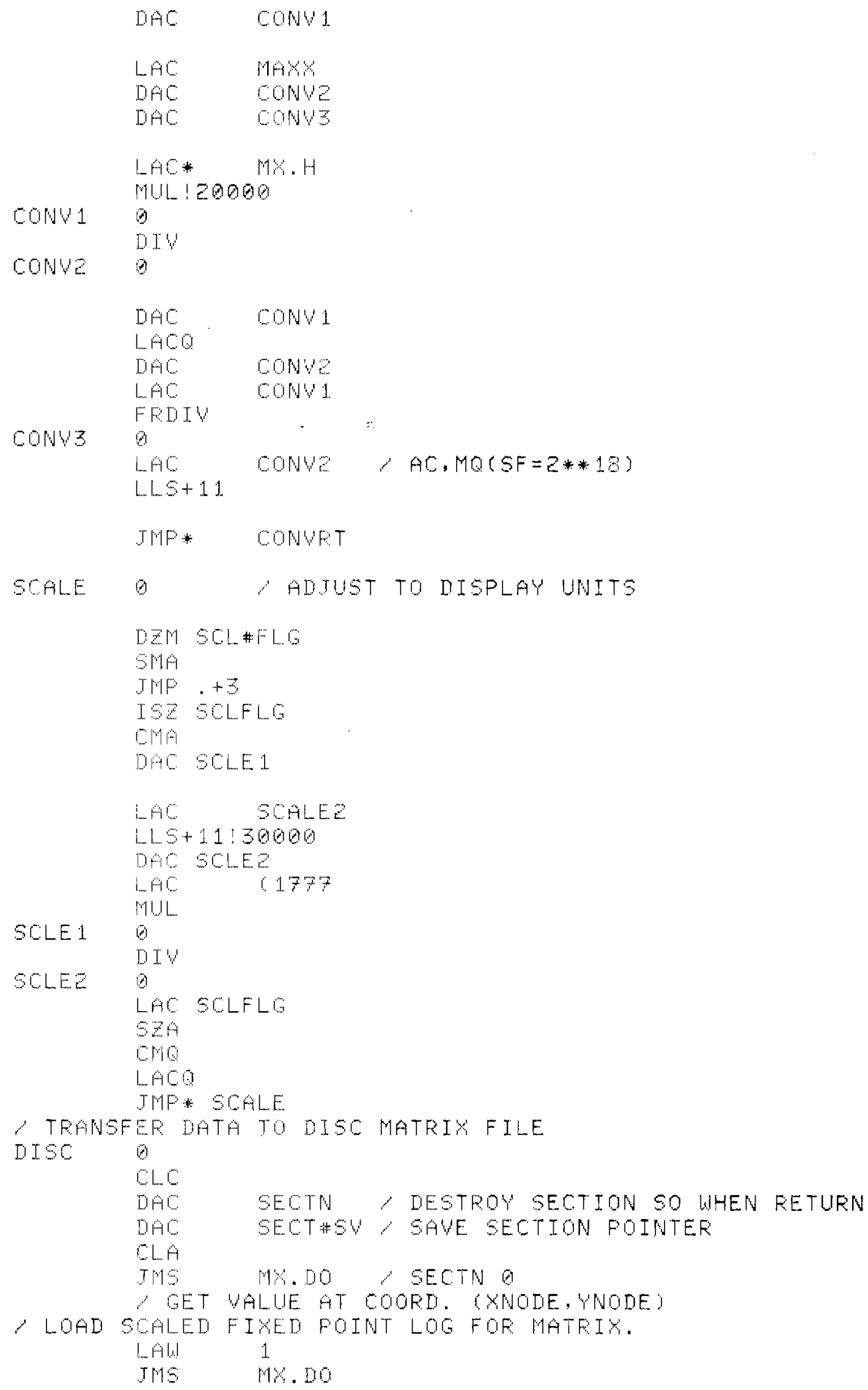




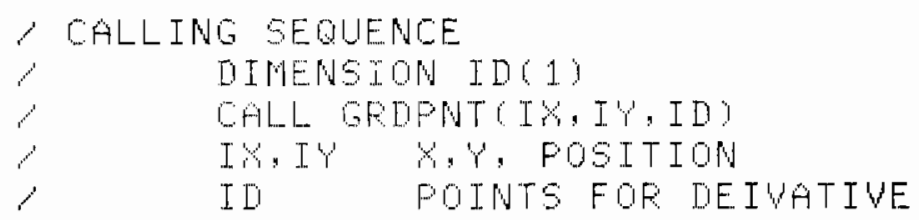

TERIUATIVE FORMIUA

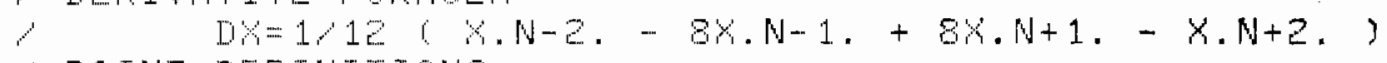

$\therefore$ POINT DEFINITIONS

\begin{tabular}{|c|c|c|c|c|c|c|}
\hline$\zeta$ & 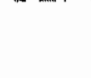 & & * 16 & $* 20$ & & \\
\hline 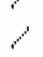 & $* 7$ & * 8 & $\begin{array}{l}* 15 \\
* 9\end{array}$ & $\begin{array}{l}* 19 \\
* \quad 10\end{array}$ & * 11 & * 12 \\
\hline$r$ & $* 1$ & * 2 & $* 3$ & * 4 & * 5 & $* 6$ \\
\hline$r$ & & & * 14 & * 18 & & \\
\hline$y$ & & & $* \quad 13$ & * 17 & & \\
\hline
\end{tabular}

. OLOEL GEDPNT, .DA

GRIPNT D

\begin{tabular}{|c|c|}
\hline & J川S* \\
\hline$x$ & 0 \\
\hline$Y^{\prime}$ & 0 \\
\hline DATA & 0 \\
\hline
\end{tabular}

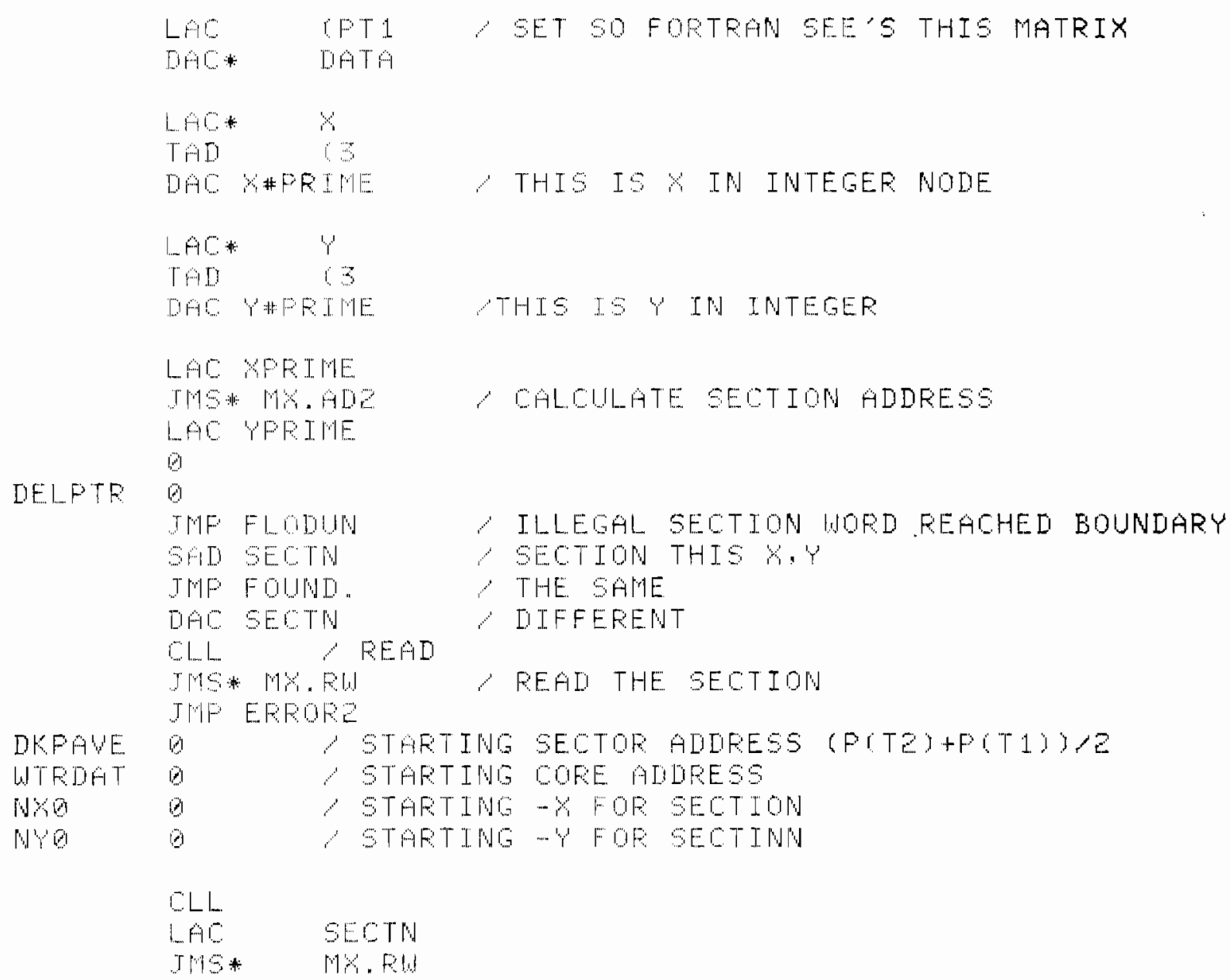




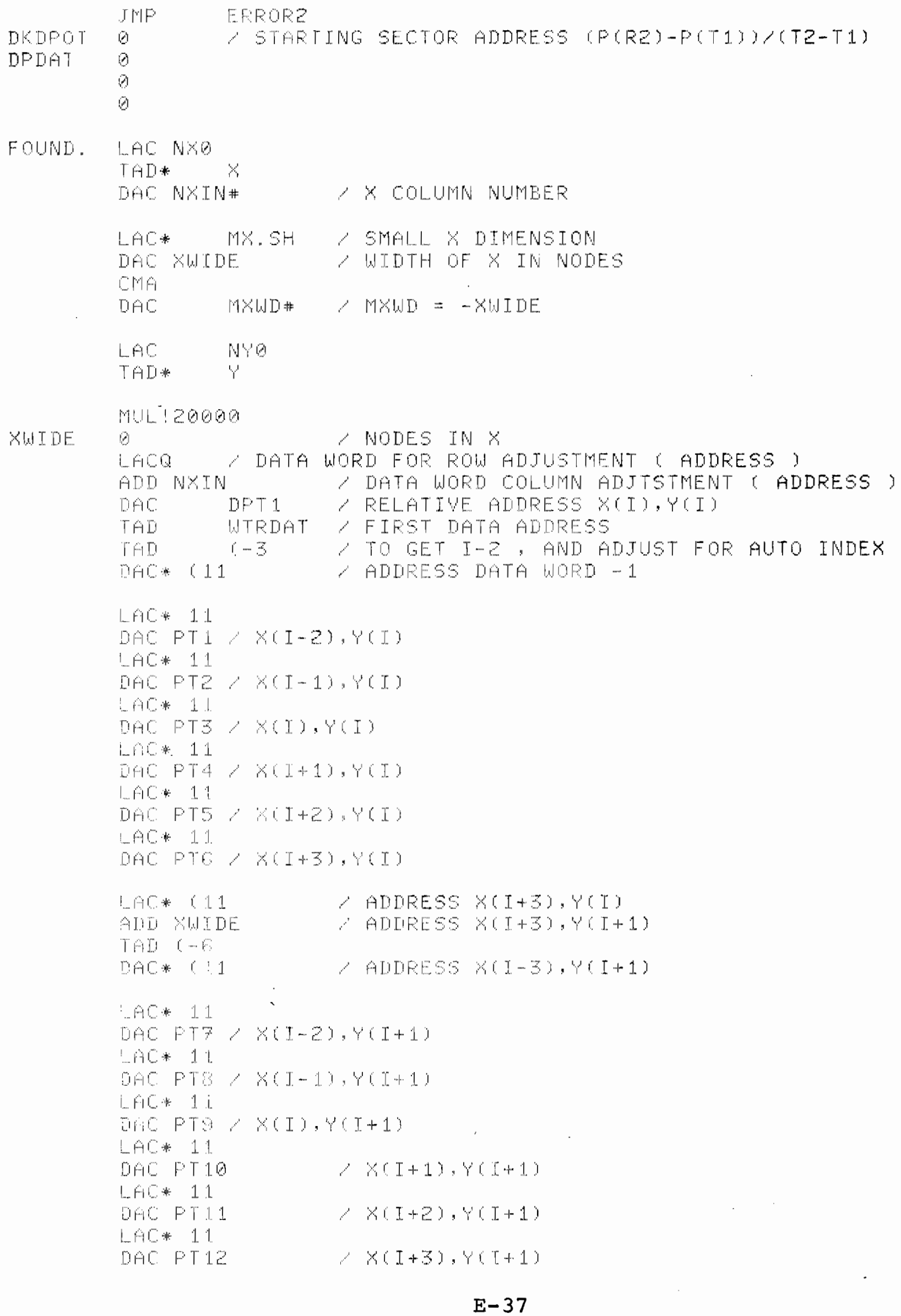




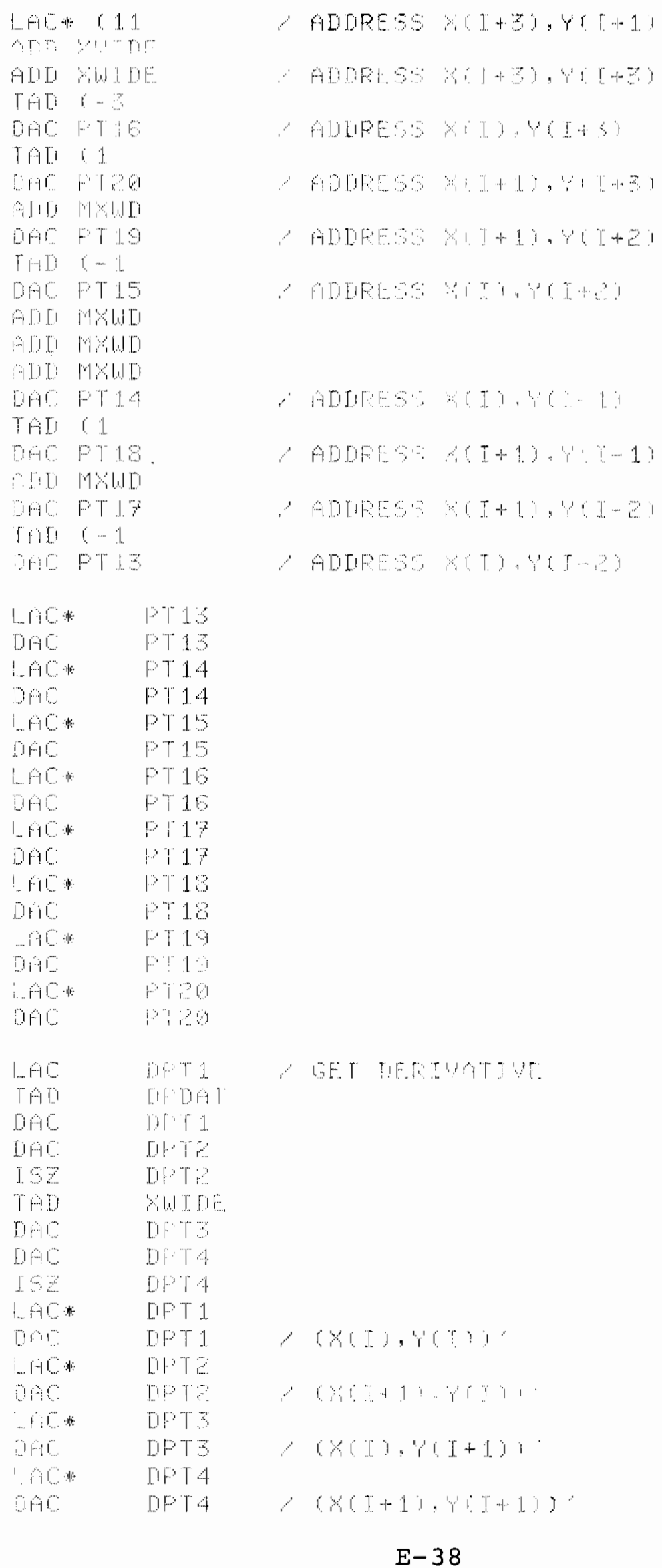




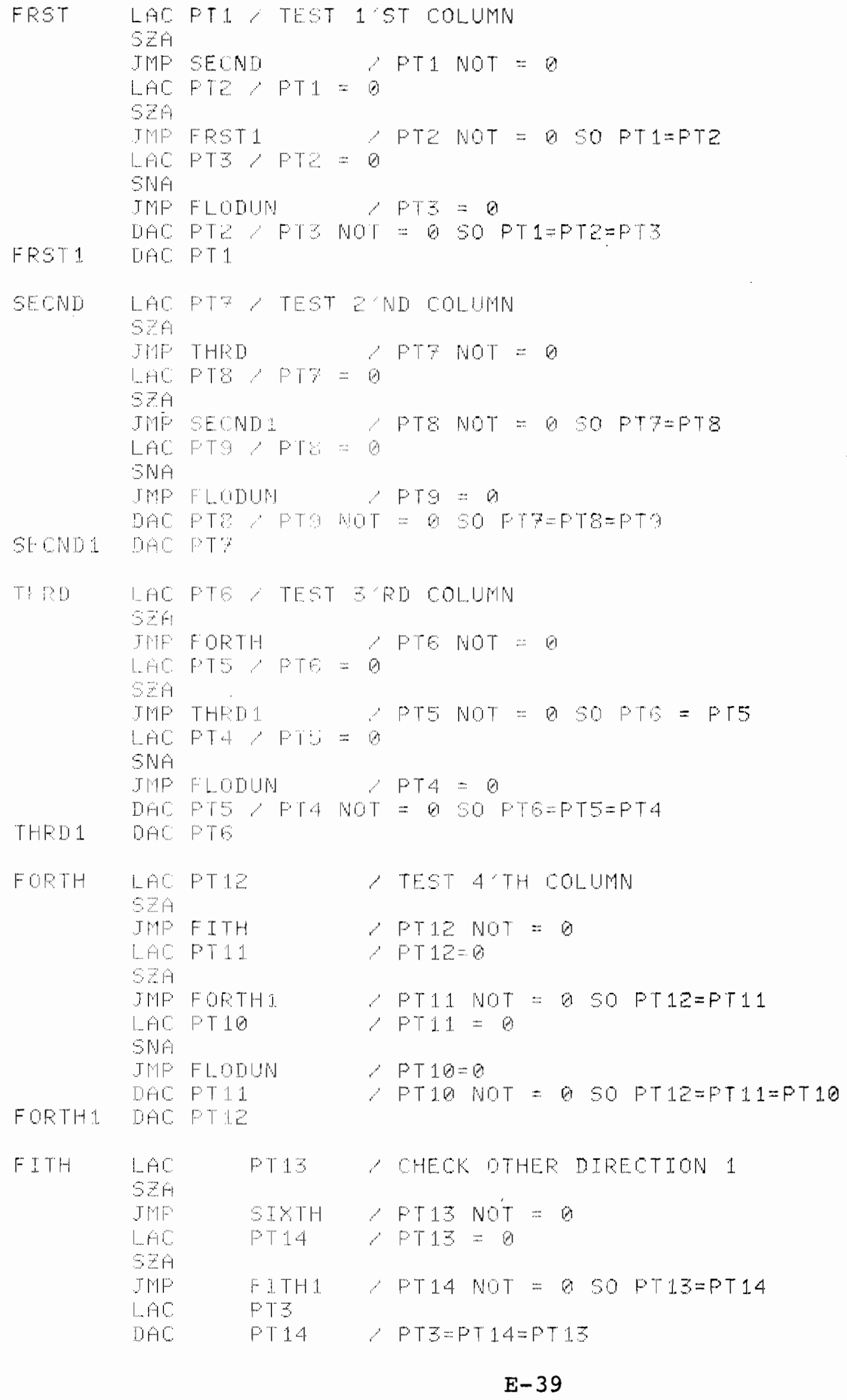




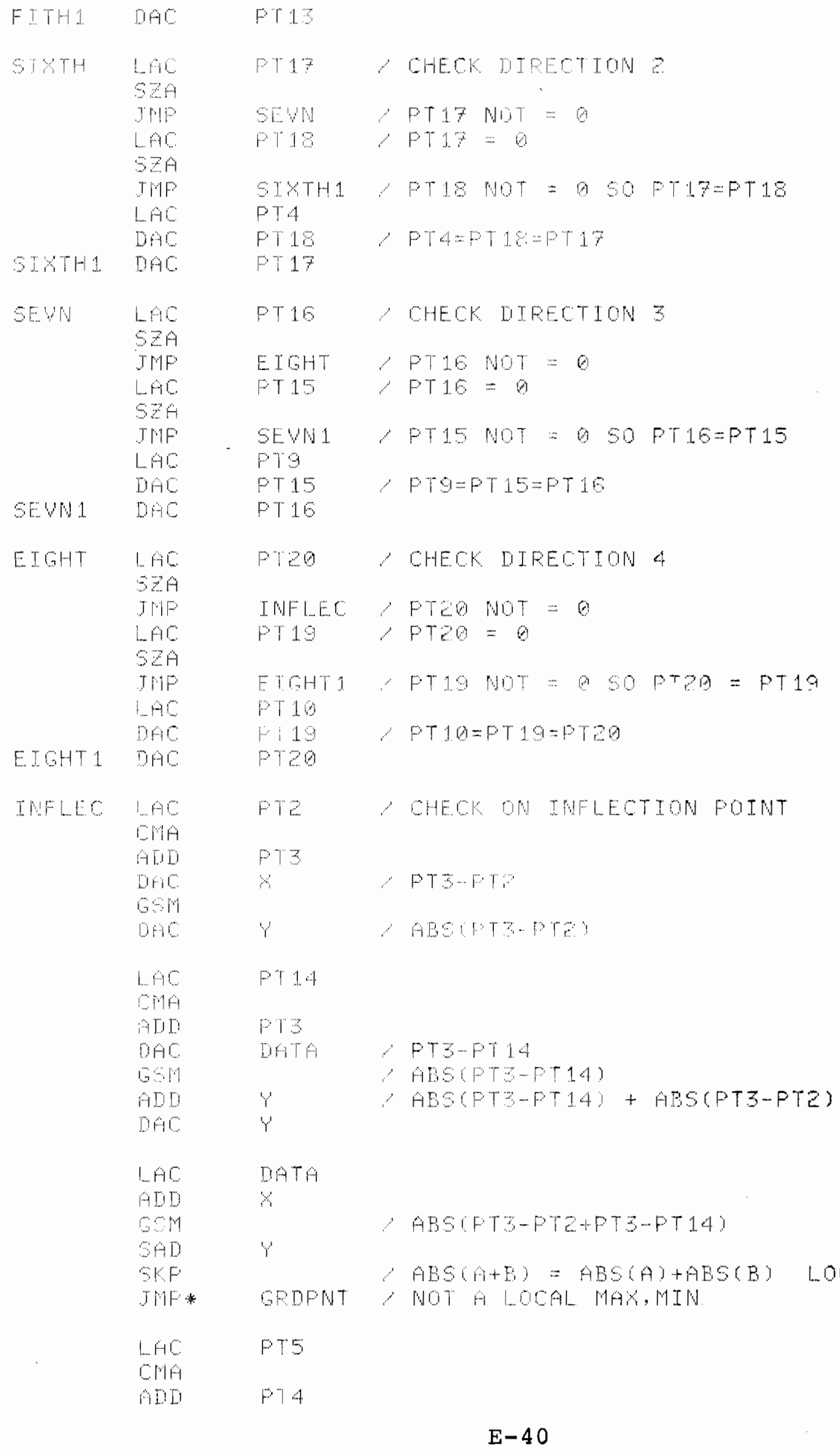




\begin{tabular}{|c|c|}
\hline $\begin{array}{l}\text { DAC } \\
\text { GeM } \\
\text { DAC }\end{array}$ & 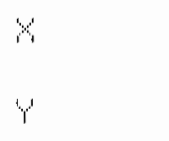 \\
\hline $\begin{array}{l}\angle A C \\
\text { CMA } \\
\text { ADD } \\
\text { DAO } \\
\text { GSU } \\
\text { ADD } \\
\text { DAC }\end{array}$ & $\begin{array}{l}\text { PA } \\
\text { DATA } \\
Y \\
Y\end{array}$ \\
\hline $\begin{array}{l}\angle A C \\
\text { AOH } \\
\text { SSH } \\
\text { SAT } \\
\text { SKF } \\
\text { JMF }\end{array}$ & $\begin{array}{l}\text { DATA } \\
x \\
Y \\
\text { GRDPBT }\end{array}$ \\
\hline $\begin{array}{l}\text { LAC } \\
\text { CMA } \\
\text { ADD } \\
\text { DAC } \\
\text { GलM } \\
\text { कAC }\end{array}$ & $\begin{array}{l}\text { PTS } \\
\text { PTS } \\
y_{1}\end{array}$ \\
\hline $\begin{array}{l}\text { LAD } \\
\text { CHA } \\
\text { ADC } \\
\text { DSO } \\
\text { ADD } \\
\text { DAD }\end{array}$ & $\begin{array}{l}\text { PT } 15 \\
\text { DT } \\
\text { DHTh } \\
y \\
y\end{array}$ \\
\hline 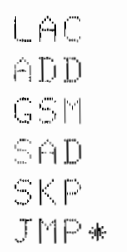 & 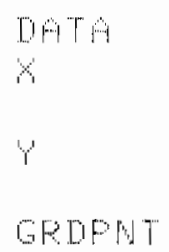 \\
\hline $\begin{array}{l}\text { AC } \\
\text { CMA } \\
\text { ADD } \\
\text { DAC } \\
\text { DSO } \\
\text { DAC }\end{array}$ & $\begin{array}{l}\text { PT11 } \\
\text { PT10 } \\
\qquad \\
Y\end{array}$ \\
\hline $\begin{array}{l}\text { LAC } \\
\text { CMA } \\
\text { ADD } \\
\text { DAC } \\
\text { GOM } \\
\text { ADD } \\
\text { DAC }\end{array}$ & $\begin{array}{l}\text { PTL } \\
\text { PTLO } \\
\text { DATH }\end{array}$ \\
\hline LAC & БөТА \\
\hline
\end{tabular}

$E-41$ 


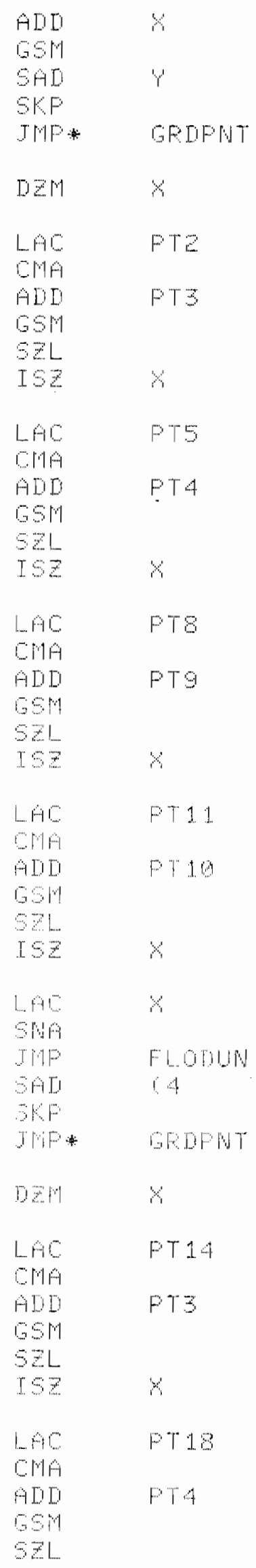




\begin{tabular}{|c|c|}
\hline$I S z^{\prime}$ & $x$ \\
\hline $\begin{array}{l}\mathrm{ACO} \\
\mathrm{CHA}\end{array}$ & PT15 \\
\hline $\begin{array}{l}A D D \\
65 \% \\
5 z^{2}\end{array}$ & FT9 \\
\hline $\operatorname{ISZ}$ & $x$ \\
\hline $\begin{array}{l}\text { LAC } \\
\text { CMA }\end{array}$ & FT 19 \\
\hline $\begin{array}{l}\text { ADD } \\
69 M \\
\text { SEL }\end{array}$ & $P T 9$ \\
\hline$I S Z$ & $x$ \\
\hline $\begin{array}{l}\text { LAC } \\
\text { SNA. }\end{array}$ & 8 \\
\hline $\mathrm{TMF}$ & FoDU LOS \\
\hline TMP & F LoTuR \\
\hline JWF* & GRDFNT \\
\hline
\end{tabular}

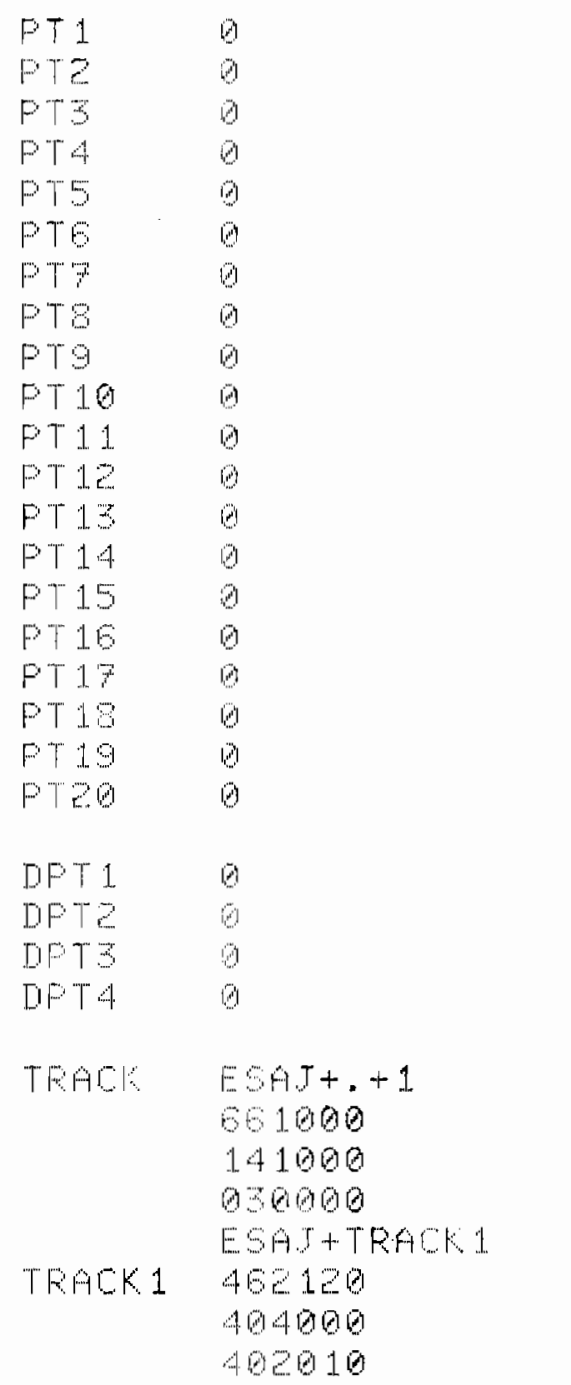




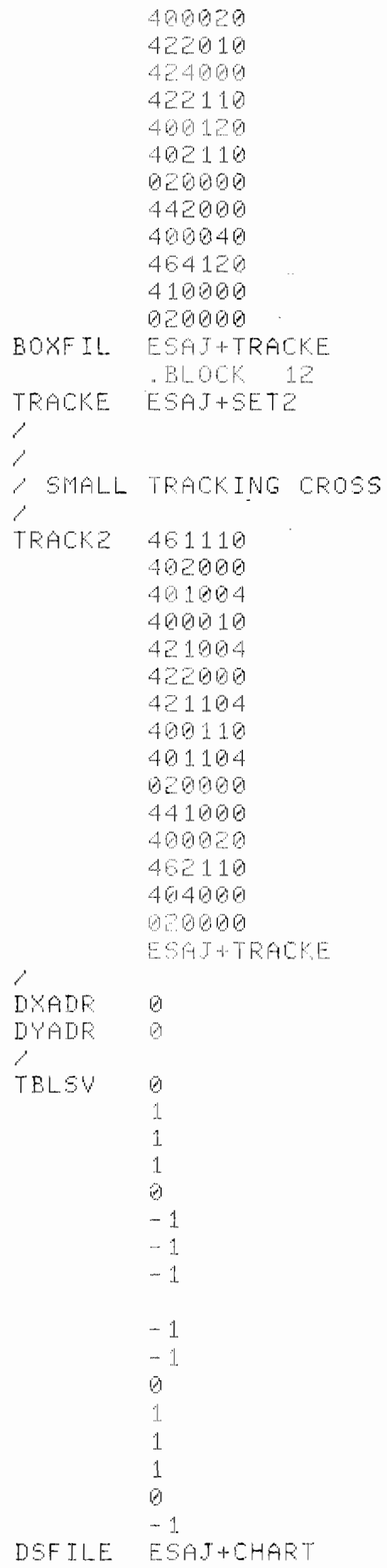




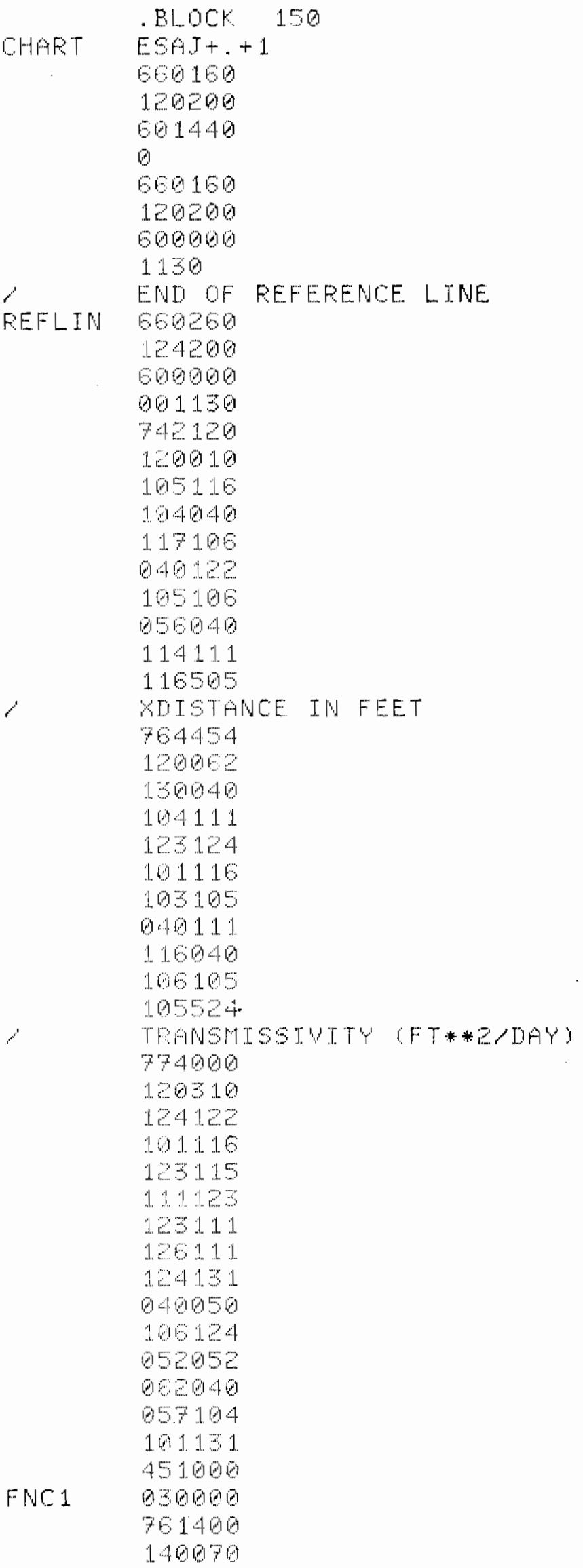




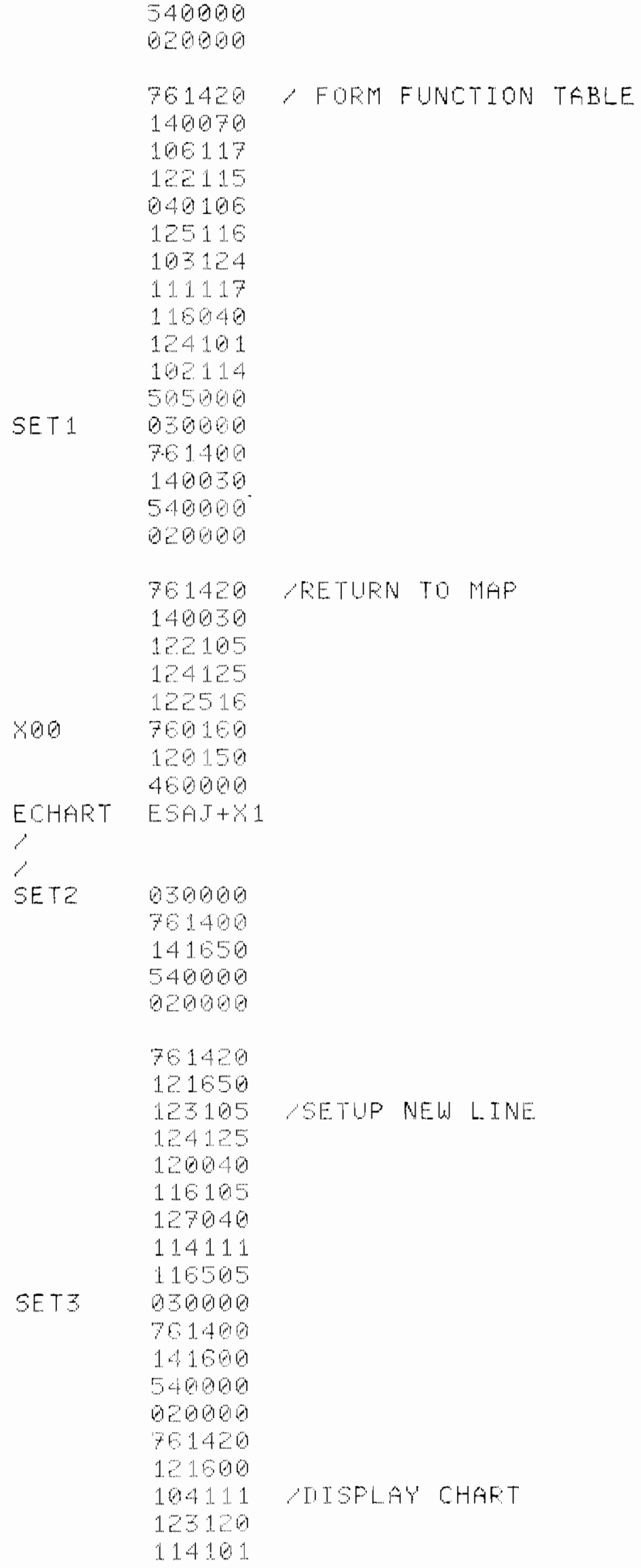




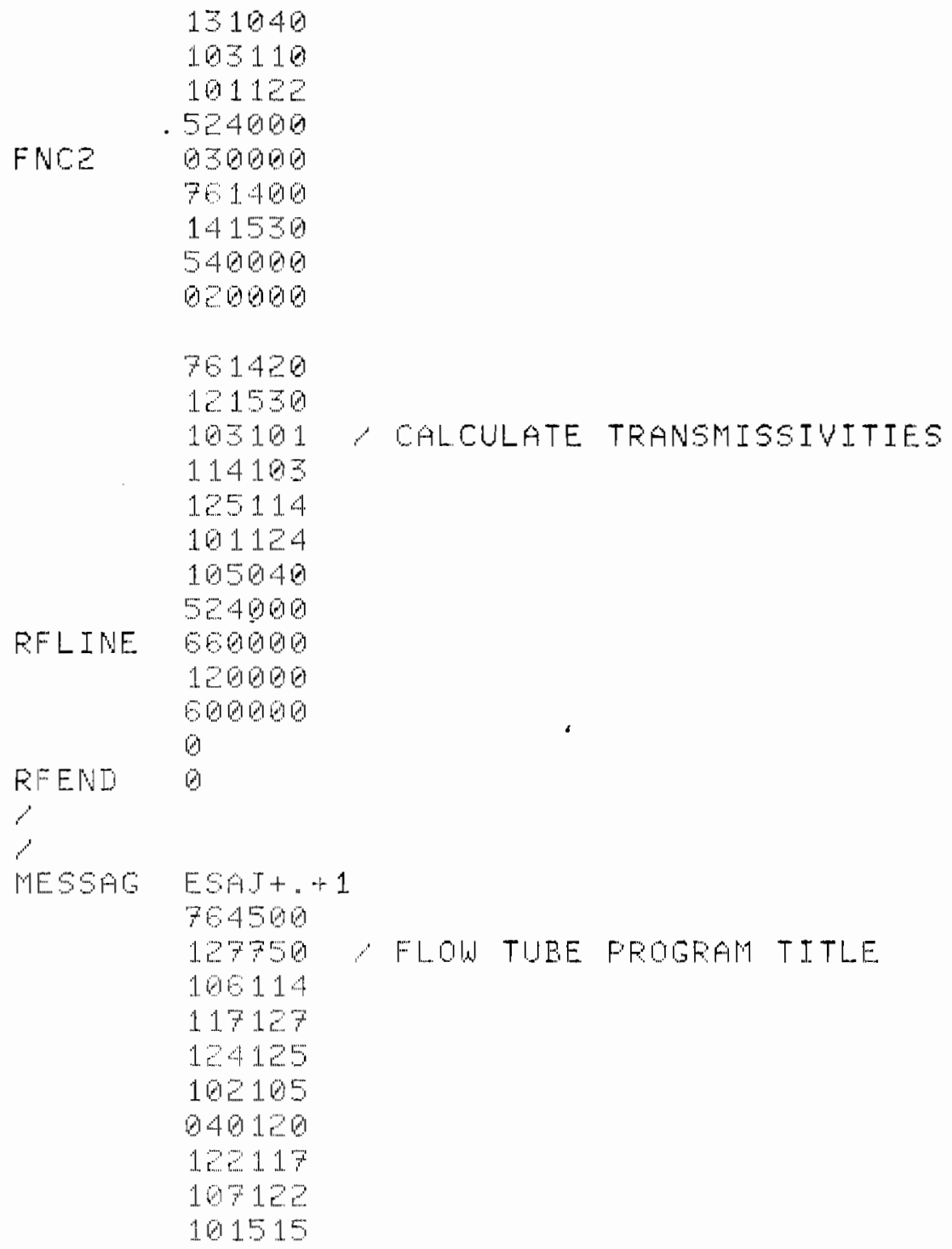

REAL 1

ESAJ+REALZ

WORIT

- AESVC 10, 35

. QETT OTGITIZE FLOTUE ORIGIN'〈4OO〉

REAL? D

$E S A T+E C H O$

A $A S 4 \mathrm{C} \quad 10,35$

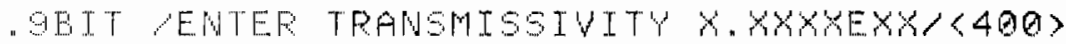

ECHO ESAT+ +3

ESAT STRMFL $-20$

76000

140010

44000

- BLOCK 17

ESAT+ECHO+ 1 


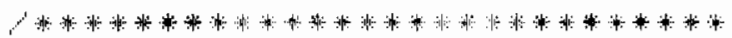

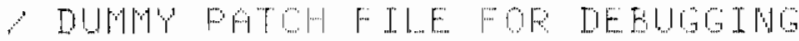

PATCH . BLOCK 20

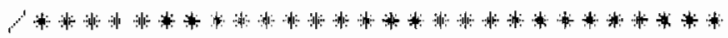

. END 


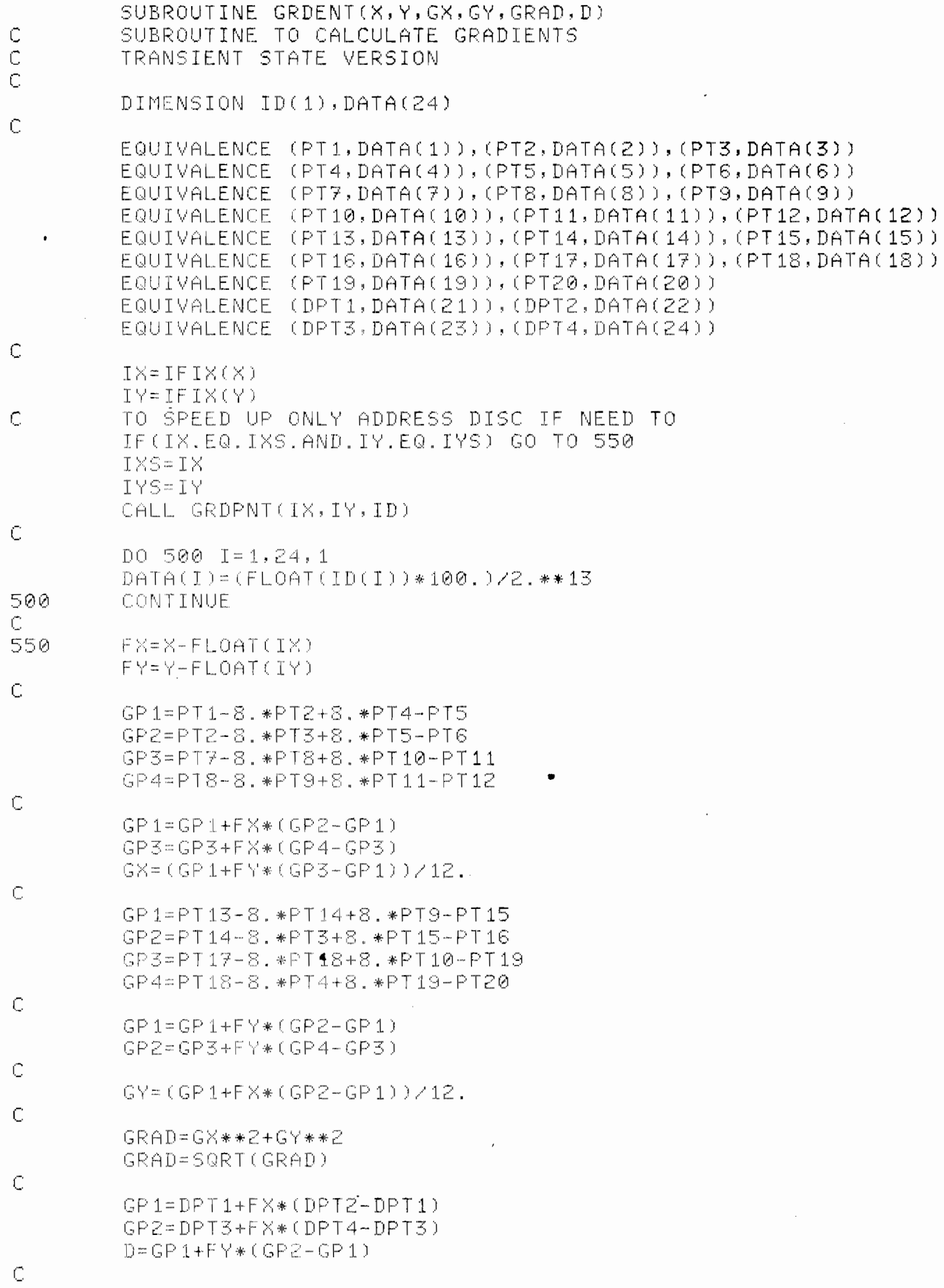

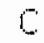

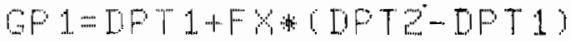

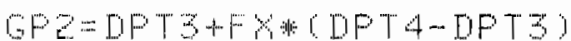

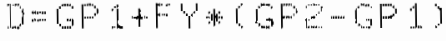

1 
RETURH

END

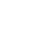

E-50 
SUEROUTIME TRANTRCIK, IX, IY, TRAH, ITCH, IY, IT, IAX, IAY, ADJST,

VERSION TO CALCULATE TRAMSIENT STATE TRANSSMISSIUITY

$T(I+1)=T(I) *(G R A D(I) * W(I))(G R A D(T+1) * 1(I+1)$

T TRANSWISIVITY

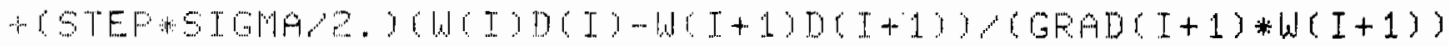

GEAD GRALERT

1.1 WTTH

STEP DISTANOE EETWEEN NODES (FEET)

SIGMA POROSITY

II DERTWATTE

IK GONTROL WORD

IX, IY X, Y POSITIOH YYX.XYK

TRAP TRANSMISEIUT'Y

ITCH DIRECTION SWITCH

IU VECTOR ADJUSTMENT

IT. TIME BETUEEN TE:T1 LAYS)

IAX, IAY ABSOLUTE $X$ Y POSITIOH DISPLAY UNITS

ADIST PEODUCT USED IN BACKWARO CALCULATION

REAL KSO, KYO,KXI,KY1

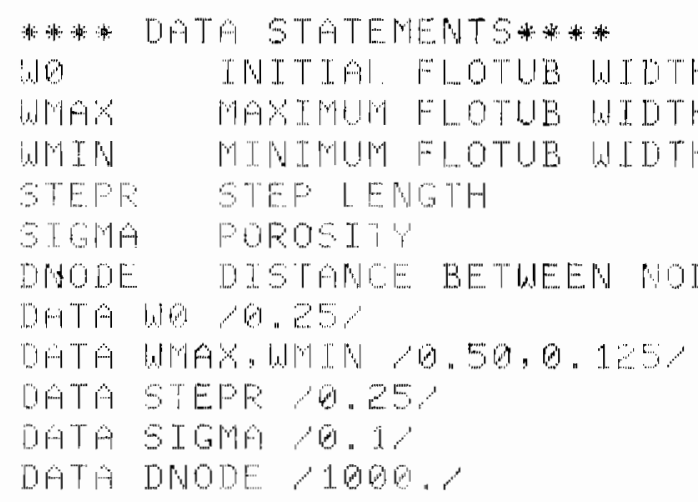

$\mathrm{C}$

HPU.

$T H=T R A P$

GFAM

$\mathrm{TP}=\mathrm{MNF}$.

C

$8 x 0=6 \%$

KYO=FY1

$30-6$

$Y 0=42$

$x_{1}=x_{3}$

$r \perp=r 3$ 


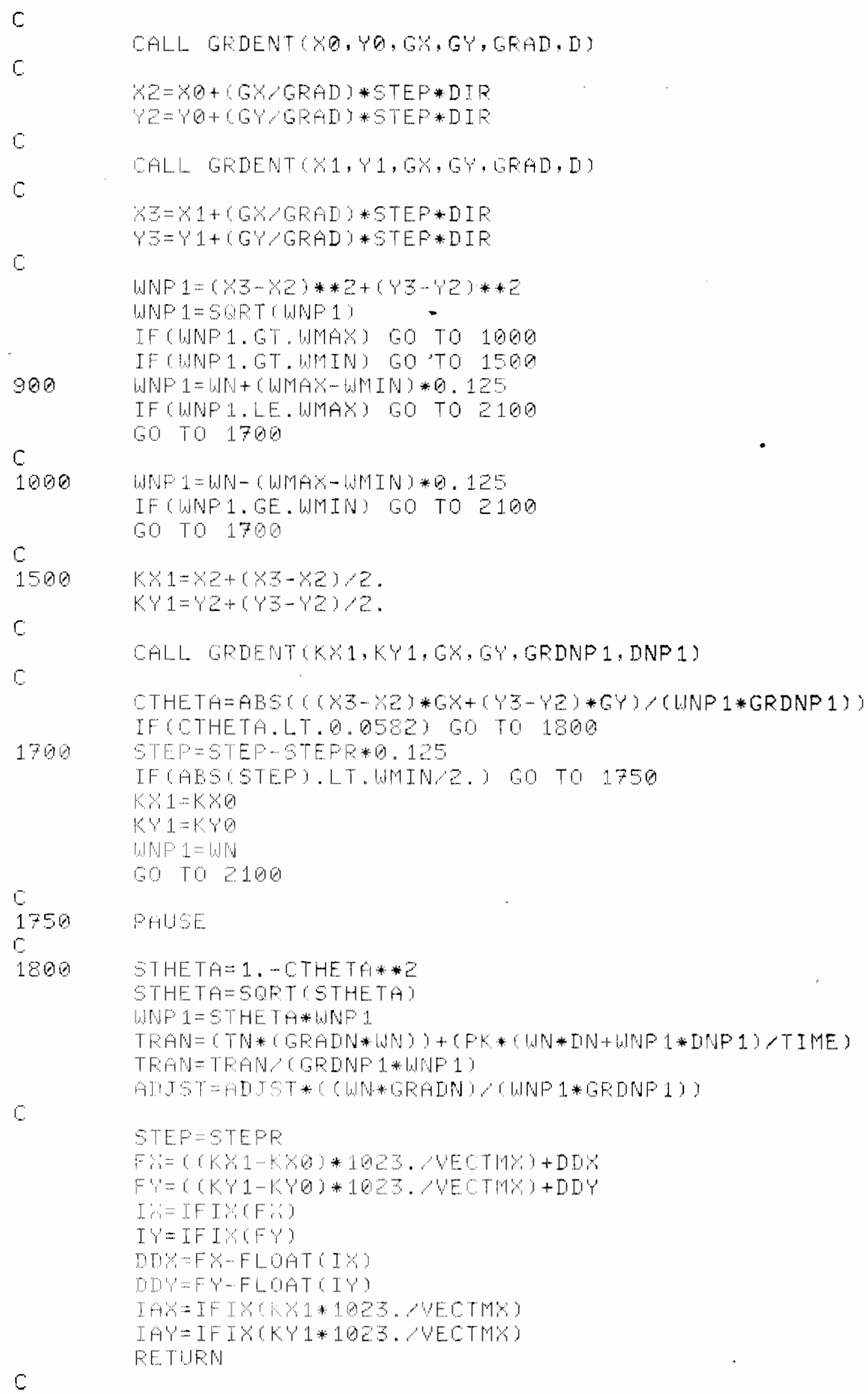




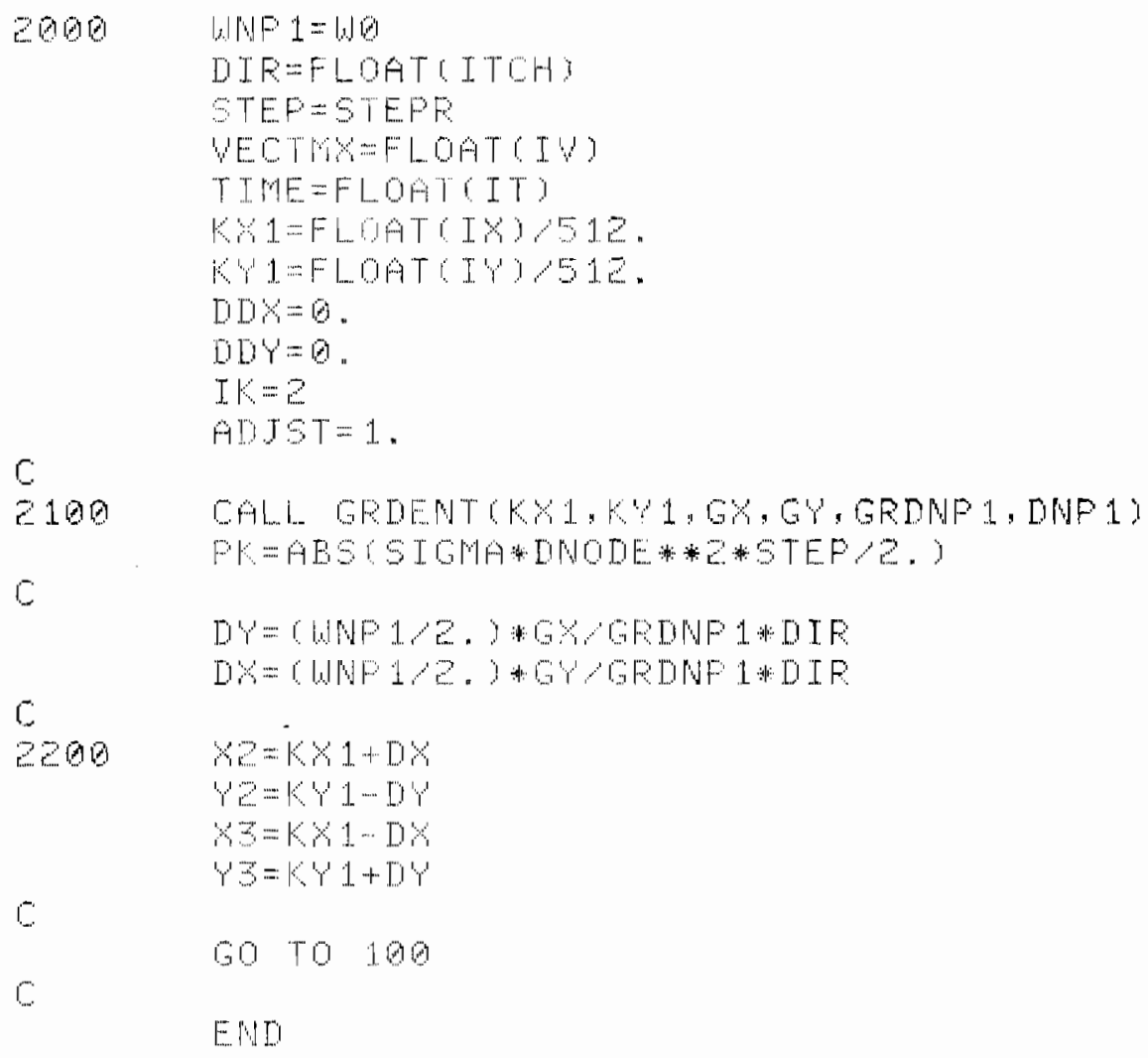




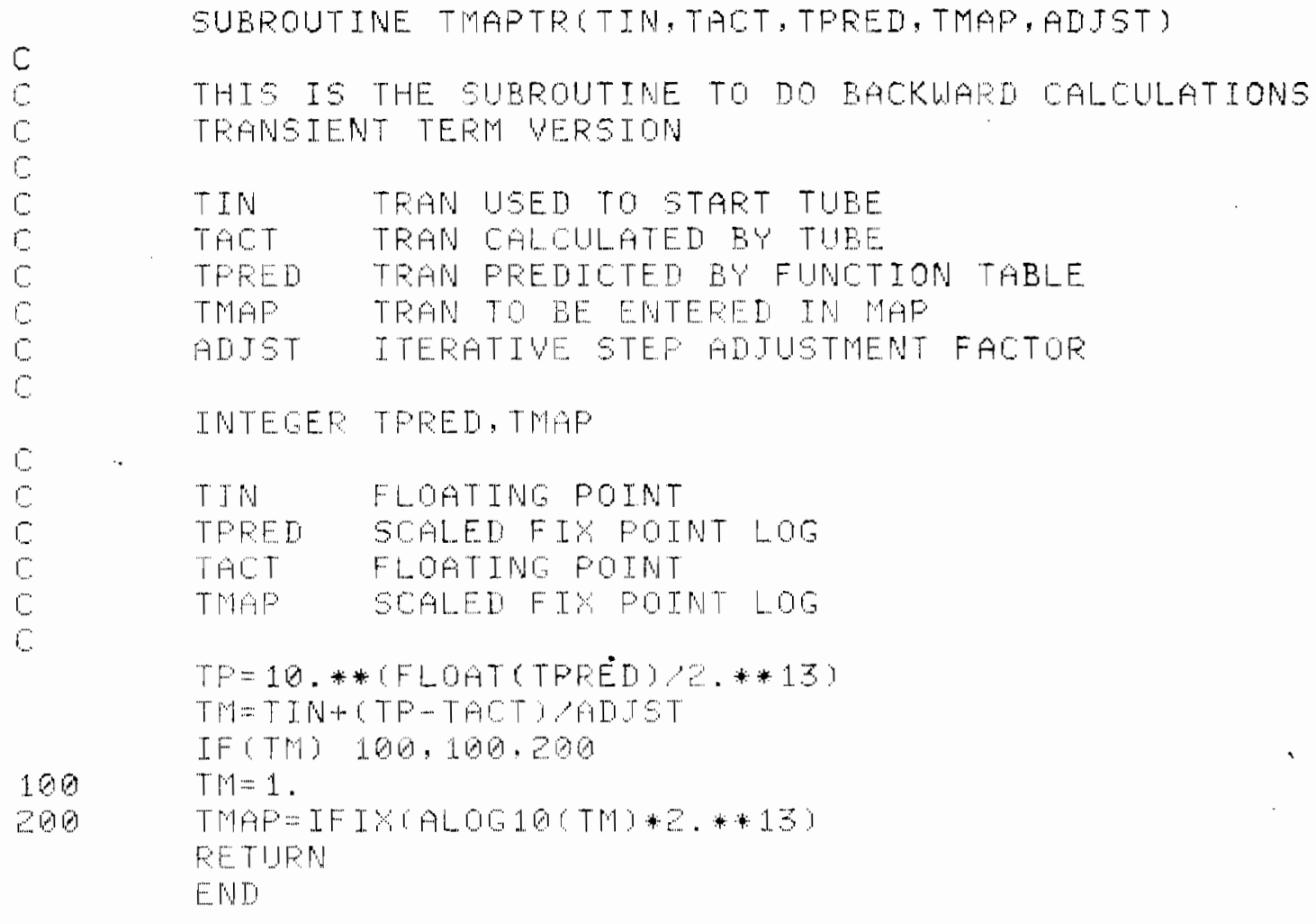




\title{
DISTRIBUTION
}

\author{
No. of \\ Copies Off-Site \\ AEC Chicago Patent Group \\ G. H. Lee \\ AEC Technical Information Center \\ Atomic Energy Commission Headquarters \\ Waste Management and Transportation \\ Germantown, Maryland 20767 \\ Owen P. Gornley \\ Chief, Waste Facilities Branch \\ Robert W. Ramsey, Jr. \\ Chief, Development Branch \\ Atomic Energy Commission Site Safety \\ Division \\ Directorate of Regulation \\ 7920 Norfolk Avenue \\ Bethesda, Maryland 20014 \\ Ronald L. Bullard \\ Chief, Environmental Specialist Branch \\ W. Gamill \\ Chief, Site Analysis Branch \\ Atomic Energy Commission \\ Division of Production \& Material Management \\ Washington, D. C. 20545 \\ William L. Lennemann \\ Chief, Chemical Process \\ James W. Pollock \\ Atomic Energy Commission \\ Idaho Falls, Idaho 83401 \\ Dr. Adrian H. Dahl \\ Chief, Environmental Science Branch \\ B. L. Schmalz




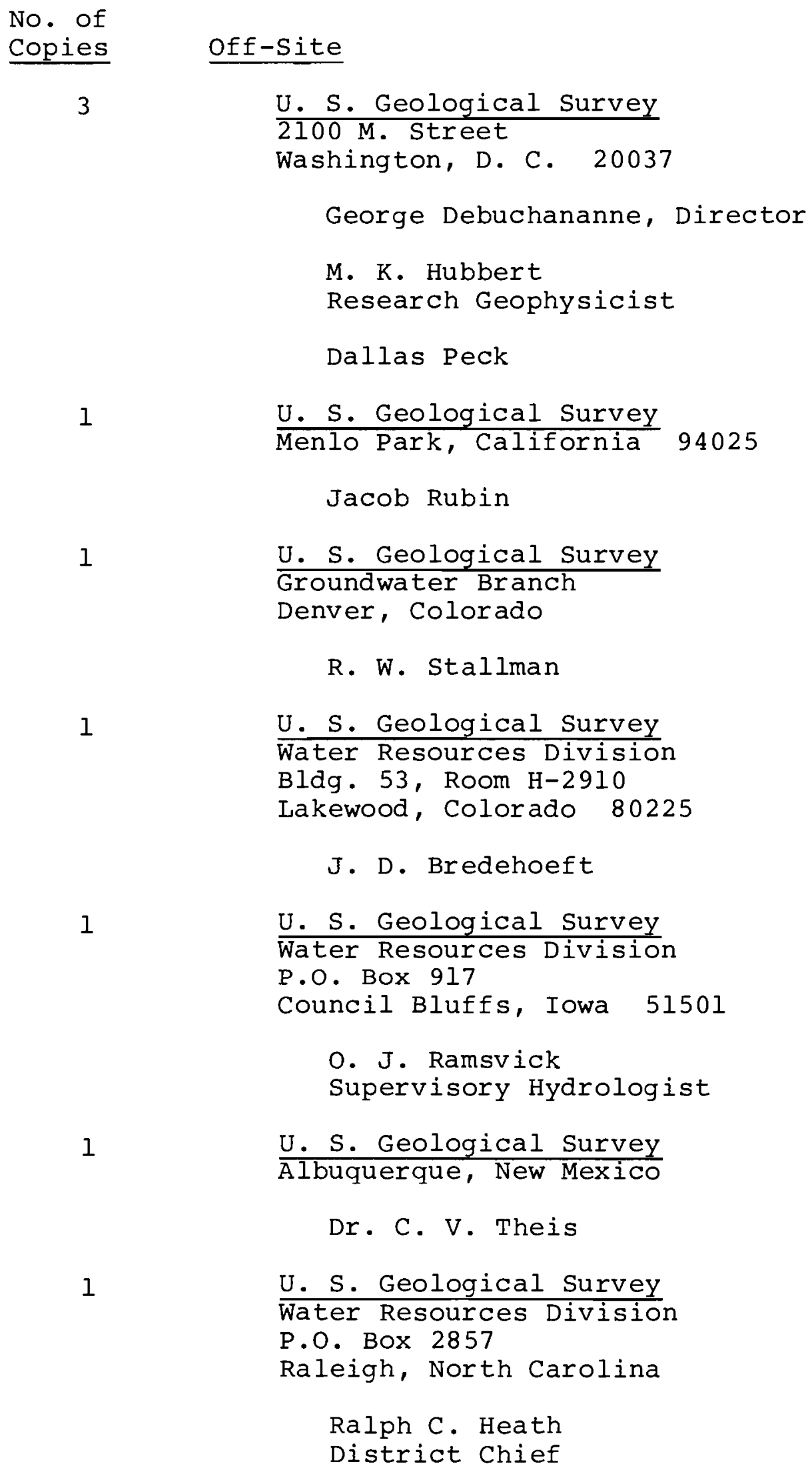

U. S. Geological Survey 2100 M. Street 
No. of

Copies

2

Off-Site

U. S. Bureau of Mines

4800 Forbes Avenue

Pittsburgh, Pennsylvania 15213

George Evans

Robert W. Van Dolah

3

U. S. Bureau of Mines

Spokane Mining Research Laboratory

N. 1430 Washington St.

Spokane, Washington 99201

Robert C. Bates

Dan Keeley

Michael M. McDonald

1

U. S. Forestry Service

Intermountain Forest and Range Experiment Station

Boise, Idaho 83702

Dr. Walter F. Meghan

Research Hydrologist

Environmental Protection Agency

Crystal Mall

Washington, D. C. 20460

Arnold Joseph

1

Environmental Protection Agency

Office of Research \& Monitoring

Edison Water Quality office

Edison, New Jersey 08817

Richard Field

1

National Water Commission

800 N. Quincy

Arlington, Virginia 22209

Dr. John S. Gladwell

1

USDA-ARS

U. S. Water Conservation Laboratory

4331 East Broadway

Phoenix, Arizona 85040

Dr. Herman Bouwer

Hydraulic Engineer 
No. of

Copies

Off-Site

1

USDA-ARS

U. S. Salinity Laboratory

P.O. Box 672

Riverside, California 92502

Dr. Jan Van Schilfgaarde, Director

1

USDA-ARS

Engineering Research Center

CSU Foothills Campus

Fort Collis, Colorado 80521

Dr. David A. Woolhiser

Hydraulic Engineer

1

USDA-ARS

Northwest watershed Research Center

P.O. Box 2700

Boise, Idaho 83701

Walter J. Rawls, Hydrologist

1

USDA-ARS

P.O. BOX 1096

Boise, Idaho 83701

J. I. Robins

1

USDA-ARS

Twin Falls, Idaho

M. E. Jensen

1

USDA-ARS

Snake River Conservation Research Center Route 1 , Box 186

Kimberly, Idaho 83341

Dr. Marvin E. Jensen, Director

1

USDA Hydrograph Laboratory

Plant Industry station

Soils Building

Beltsville, Maryland 20705

H. N. Holton, Director

1

USDA-ARS

Plant Industry station

Beltsville, Maryland 20705 
No. of

Copies

Off-Site

Dr. Donald L. Brakensick

Chief Engineer

USDA Sedimentation Laboratory

P.O. Box 30

Oxford, Mississippi 38655

A. R. Robinson, Director

1

USDA-ARS

Southern Region

Stoneville, Mississippi 38776

John C. Stephens, Director

1

USDA-ARS

North Central Watershed Research Center P.O. Box 916

Columbia, Missouri 65201

Dr. C. R. Amerman

Hydraulic Engineer

1

USDA-ARS

North Appalachian Experimental watershed Coshocton, Ohio 43812

Dr. W. M. Edwards

Soil Scientist

1

USDA

P.O. Box 400

Chickasha, Oklahoma 73018

Harry B. Pionke

1

USDA-ARS

Northeast watershed Research Center

Pennsylvania State University

111 Research Building 3

University Park, Pennsylvania 16802

Dr. A. S. Rogowski

Soil Scientist

National Academy of Science

U. S. National Committee for the IHD 2101 Constitution Avenue

Washington, D. C. 20418

Dr. Leo Heindl

Executive Secretary 
No. of

Copies

Off-Site

1

National Academy of Science

Committee on Radioactive Waste Management National Research Council

2101 Constitution Avenue

Washington, D. C. 20418

Dr. Cyrus Klingsberg

Technical Secretary

1

National Science Foundation

Division of Advanced Technology Application 1800 6th Street N.W.

Washington, D. C. 20530

Ray Zahradnik

1

U. S. Naval Civil Engineering Laboratory

Port Hueneme, California 93043

Dr. James J. Der

2

Robert S. Kerr Water Research Center

P.O. BOX 1198

Ada, Oklahoma 74820

Dr. J. W. Keeley

Marion R. Scalf

1

Alabama Geologic Survey

P.O. Drawer 0

University, Alabama 35486

Phil Lammereaux

State Geologist

1

Southwest Rangeland Watershed Research

Center

442 E. Seventh Street

Tucson, Arizona 85705

Dr. R. Smith

1

California Dept. of Water Resources

Sacramento, California

Helen J. Peters

Distr -6 
No. of

Copies

1

1

1

1

1

1

1

2
Off-Site

Idaho Bureau of Mines \& Geology

University of Idaho

Moscow, Idaho 83843

Dr. Roy E. Williams

Idaho Dept. of Water Administration

State House, Ânnex 2

Boise, Idaho 83707

Keith R. Higginson, Director

Idaho water Resources Board

Boise, Idaho

R. R. Lee

Illinois State water Survey P.O. Box 232

Urbana, Illinois 61801

Keros Cartwright

State Geological Survey of Kansas University of Kansas

Lawrence, Kansas 66044

Dr. John C. Ha lepaska

Chief, water Resources Section

State of Maine

Environmental Improvement Commission Augusta, Maine 04330

Steven D. Freedman

St. Anthony Falls Hydraulic Laboratory Mississippi River at 3rd Avenue East Minneapolis, Minnesota 55414

Professor C. Edward Bowers

Desert Research Institute

Center for water Resources Research

Reno, Nevada

Dr. Clinton Case

G. B. Maxey

Distr -7 
No. of

State of North Carolina Groundwater Division Water and Air Resources Raleigh, North Carolina 27611

Harry M. Peek, Chief

1

Water Quality Management Laboratory Rt. 2, Box 322A Durant, Oklahoma 74701

V. L. Hauser

1

State of oregon

516 Public Service Bldg. Salem, Oregon 97310

Chris wheeler State Engineer

1

Texas Water Development Board Systems Engineering Division P.O. Box 13087 Austin, Texas 78711

Lial F. Tischler

Agencies for Environmental Conservation Montpelier, Vermont 05602

Dr. Martin L. Johnson Secretary

1

State of Washington

Department of Ecology

P.O. Box 829

Olympia, Washington 98504

John R. Raymond

1

Washington State Water Research Center Pullman, washington 99163

James Crosby, III Geologist

Atomic Industrial Forum, Inc. 475 Park Avenue South New York, New York 10016

Gerald V. Halvorsen Environmental Projects Manager 
No. of

Copies

1

1

1

1

1

1

1

2
Off-Site

Computer Sciences Corp.

Federal Building

Richland, Washington 99352

R. W. Nelson

Sandia Laboratories

Division 5166

Albuquerque, New Mexico 87115

Fred Norwood

Arthur M. Piper

\#3 Sonoma Lane

Carmel, California 93921

Alyeska Pipeline Service Co. 2100 Travis street

Houston, Texas 77002

Dr. Maurice D. Veatch

E. I. duPont denemours \& Co., Inc.

Savannah River, South Carolina

J. C. Corey

University of Arizona

Office of Arid Lands Studies

1201 East Speedway Blvd.

Tucson, Arizona 95719

Dr. Ken Faster

University of Arizona

Dept. of Agriculture Chemistry and Soils

Tucson, Arizona 85721

Dr. Gordon Dutt

University of Arizona

Dept. of Hydrology and water Resources

200-E old Psychology

Tucson, Arizona 95721

Dr. A. K. Tyagi

Dr. Chester C. Kisiel 
University of Arizona

Tucson, Arizona 95721

E. S. Simpson

A. W. Warrick

University of California Berkeley, California 94720

Dr. Warren Kauffman

P. A. Witherspoon

Dept. of Civil Engineering

University of California at Davis Dept. of Water Sciences and Engineering Davis, California 95616

J. N. Luthin

Don Nielson

Dr. Verne H. Scott

University of California at Riverside College of Biological \& Agricultural Sciences Riverside, California 92507

Dr. N. T. Coleman

Stanford University Department of Civil Engineering stanford, California 94305

Paul Kruger

Henry J. Ramey

J. B. Franzini

Stanford University School of Earth Sciences Dept. of Geology stanford, California 94305

E. Aguado 
No. of

Copies

1

1

2

1

1

1

1

1
Off-Site

Georgia Institute of Technology School of Civil Engineering

Atlanta, Georgia 30332

Dr. J. R. Wallace

University of Idaho

Moscow, Idaho 83843

Dr. Delbert Fitzsimmons

Head, Agricultural Engineering

University of Illinois

Urbana, Illinois 61801

A. Klute

Professor Ven Te Chow

Hydrosystems Laboratories

Purdue University Dept. of Agricultural Engineering Wast LaFayette, Indiana 47907

Emru D. Millette

Iowa State University

Ames, Iowa

D. Kirkham

University of Kansas

Water Resources Institute

Lawrence, Kansas 66044

Don W. Green

University of Kentucky

Department of Mechanical Engineering

Lexington, Kentucky 40506

C. T. Hahn

Harvard University

School of Public Health

Boston, Massachusetts 02138

Dr. D. W. Moeller 
No. of

Copies

1

1

1

2

1
Off-Site

Massachusetts Institute of Technology 77 Massachusetts Avenue Room 48-263

Cambridge, Massachusetts 02139

Dr. Donald Harleman

University of Minnesota Department of Geology and Geophysics 2D Pillsbury Hall

Minneapolis, Minnesota 55455

Hans Olaf Pfannkuch

University of Minnesota Department of Civil Engineering Minneapolis, Minnesota 55418

H. Steforn

University of Nebraska School of Civil Engineering Lincoln, Nebraska 68503

Mark J. Hammer

Warren Viessman, Jr.

Princeton University

School of Engineering/Applied Science Department of Civil and Geological

Engineering

Princeton, New Jersey 08540

Dr. Robert w. Cleary

Princeton University

Department of Civil \& Geological

Eng ineering

Princeton, New Jersey 08540

R. J. M. DeWiest

G. F. Pinder

New Mexico State University Department of Agronomy

P.O. BoX 3Q

Las Cruces, New Mexico 88001

P. J. Wierenga 
No. of

Copies

1

Off-Site

State University of New York at Buffalo Dept. of Mechanical Engineering

Buffalo, New York 14214

Ralph T. Cheng

1

State University of New York Dept. of Civil Engineering Buffalo, New York 14214

Dr. Ralph Rumer

2

Cornell University

School of Civil Engineering B22 Bailey Hall

Ithaca, New York 14850

W. H. Brutsaert

Winton Covey

2

Oregon State University

Corvallis, Oregon 97331

Dr. Larry Boersma

Professor of Soils

Dr. Royal H. Brooks

Professor of Agricultural Engineering

1

Drexel Institute of Technology

Philadelphia, Pennsylvania 19104

Irwin Remson

1

Vanderbilt University

Nashville, Tennessee 37203

Dr. Frank L. Parker

1

Utah State University

College of Engineering

Dept. of Agriculture and Irrigation Engineering

Logan, Utah 84321

Dr. L. G. King 
No. of

Copies

Off-Site

1

Utah State University

Utah water Research Laboratory

College of Engineering

Logan, Utah 84321

J. Paul Riley

1

Utah State University

Logan, Utah 84321

Dr. Roland $W$. Jeppson

Associate Prof. of Civil Engineering

1

Utah State University

Logan, Utah 84321

C. G. Clyde

1

University of Wyoming

Laramie, Wyoming 82070

Dr. Paul A. Rechard

Director, Water Resources Research

Institute

2

Washington State University

Dept. of Civil Engineering

148 Sloan Hall

Pullman, Washington 99163

Dr. Donald L. Bender

Dr. David Higgins

2

Washington State University

Dept. of Agronomy \& Soils

Pullman, Washington 99163

Dr. Walter H. Gardner

Dr. Eugene Thompson

1

Washington State University

Albrook Hydraulic Laboratory

Pullman, Washington 99163

Dr. John F. Orsborn, Head 


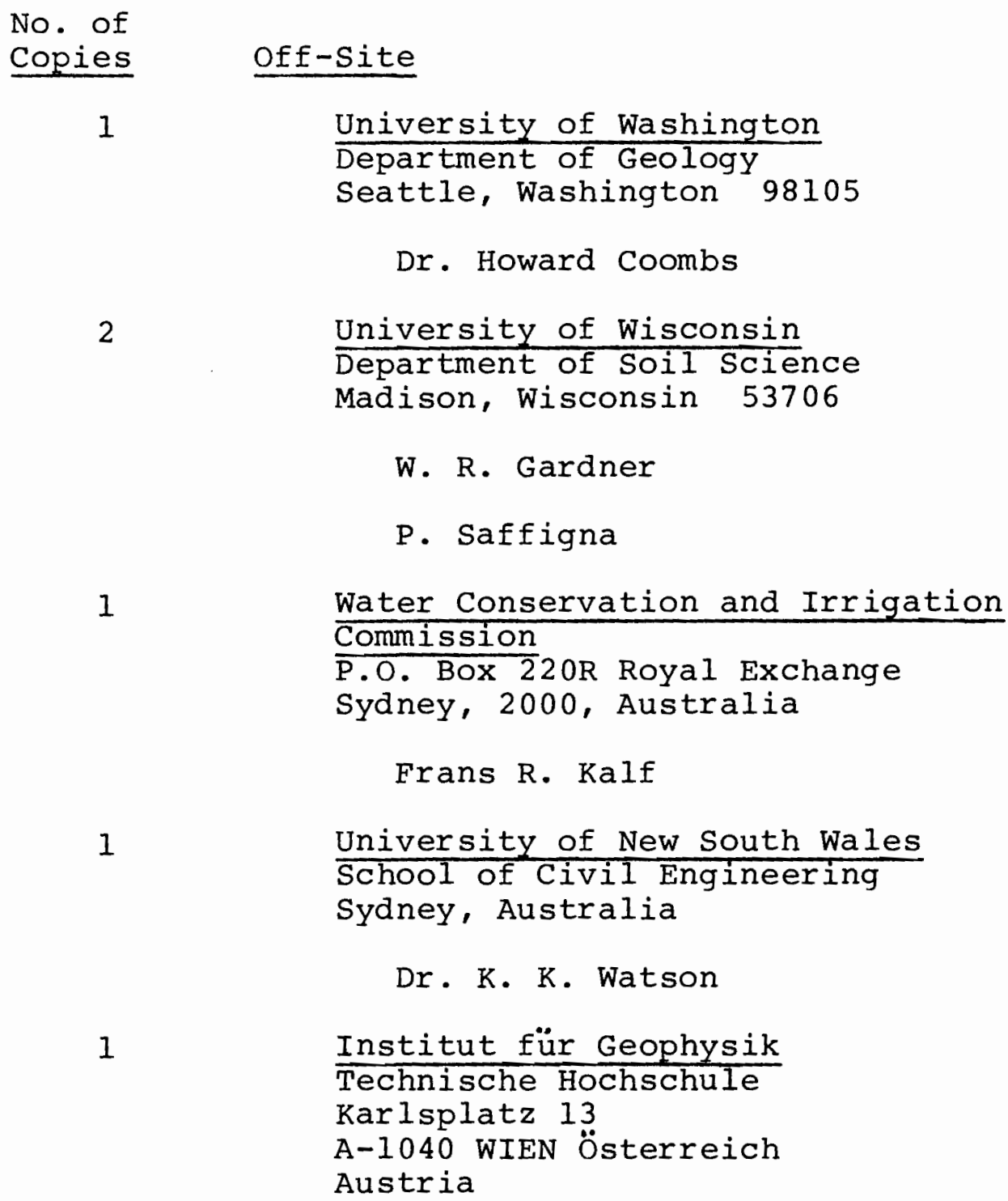

Institut für Geophysik Technische Hochschule KarIsplatz 13

A-1040 WIEN Österreich Austria
A. E. Scheidegger

1

University of British Columbia Vancouver, B. C., Canada

Dr. Allen Freeze

Dept. of Mines and Natural Resources Water Resources Branch 693 Taylor Avenue Winnipeg 9, Manitoba, Canada

Frank W. Render 


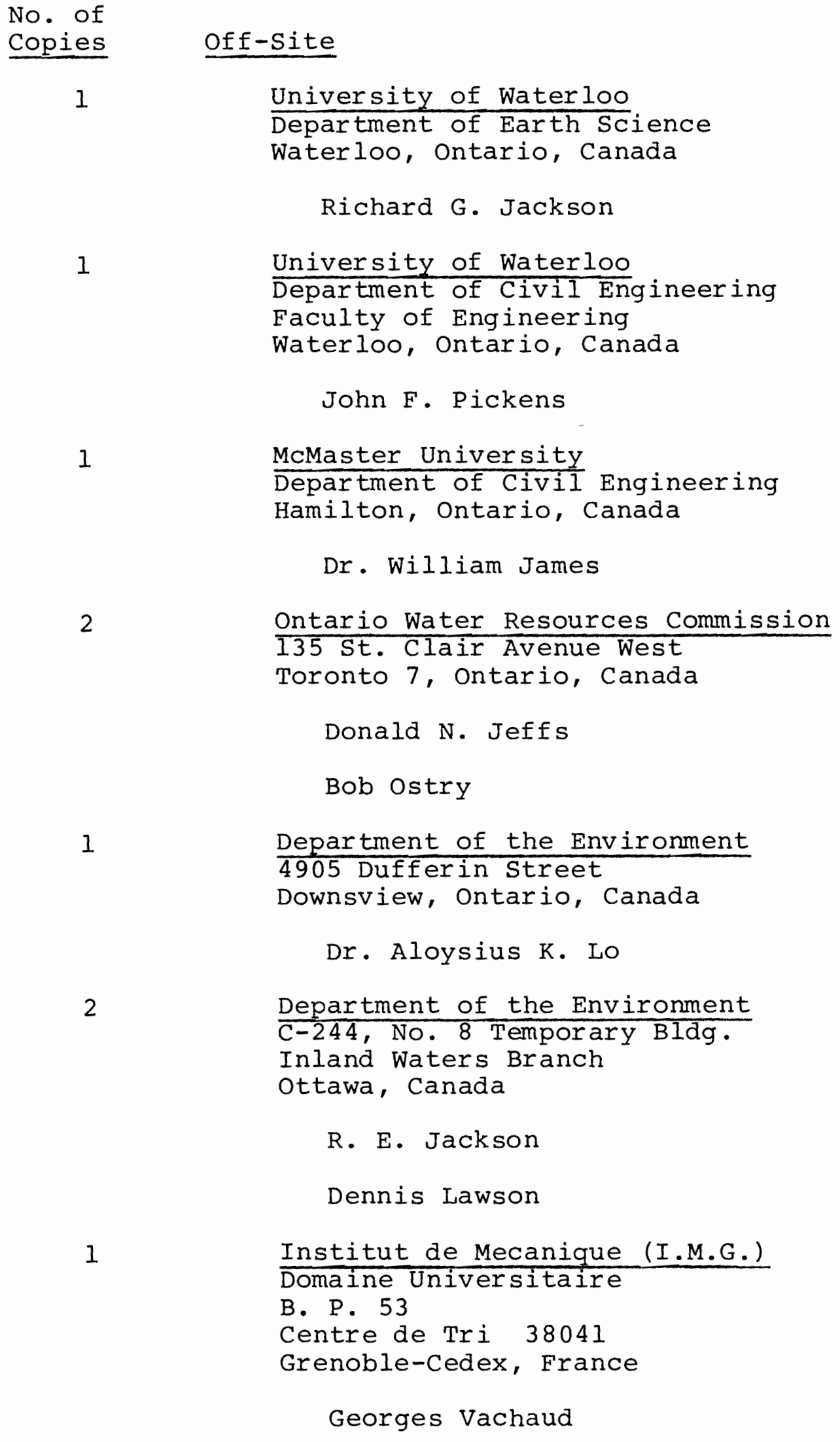

Department of the Environment 4905 Dufferin street

Department of the Environment C-244, No. 8 Temporary BIdg. Inland Waters Branch

Ottawa, Canada

$$
\text { R. E. Jackson }
$$

Dennis Lawson

Institut de Mecanique (I.M.G.) Domaine Universitaire

B. P. 53

Centre de Tri 38041

Grenoble-Cedex, France

Georges Vachaud 
No. of

Copies

1

1

1

3

1

1

1
Off-site

M. R. Engineering College

Jaipur-302004, India

Dr. R. D. Verma

Prof. of Civil Engineering

University College

Upper Merrion Street

Dublin, 2 Ireland

Dr. James C. I. Dooge

Professor of Civil Engineering

Department of Mechanical Engineers, I.I.T. Kharagkur,

S.E. RY, India

R. G. Mokadom

Technion-Israel Institute of Technology Haifa, Israel

J. Bear

Shragga Irmay

Dan Zaslavsky

Agricultural Research Organization The Volcani Center

The Institute of Soils and water

P.O.B. 6

Bet Dagan, Israel

Shlomo P. Neuman

Food \& Agriculture Organization of the UN Land and Water Development Division Water Resources \& Development Service Via Delle Terme Dl Caracalla

00100-Rome, Italy

R. G. Thomas

$\frac{\text { Kyoto University }}{\text { Kyoto, Japan }}$

Yoriteru Inoue 
No. of

Copies

Off-site

1

Explorade En Produktie Laboratorium c/o Koninklijke/Shell

Volmerlaan 6

Ritswijk (Z.H.), The Netherlands

A. M. Schaaps

1

University of Canterbury, N.Z. Dept. of Civil Engineering

Christ Church 1 , New Zealand

Bruce W. Hunt

On-Site Hanford

1

AEC/RI Patent Attorney

R. M. Poteat

AEC Richland Operations Office

O. J. Elgert

J. L. Rhoades

P. J. Holsted

E. B. Jackson

P. G. Rhoades

R. B. Goranson

Atlantic Richfield Hanford Co.

G. E. Backman

G. L. Borsheim

D. J. Brown

L. E. Bruns

M. H. Campbell

W. M. Harty

R. E. Isaacson

(2)

L. M. Knights

C. W. Malody

B. J. McMurray

M. C. Metz

H. P. Shaw

A. E. Smith

P. W. Smith

W. J. Van Slyke

ARHCO File

G. A. Nicholson 
No. of

Copies

On-Site Hanford

3

Westinghouse Hanford Co. HEDL

R. B. Hall

29

Battelle-Northwest

D. B. Cearlock

J. P. Corley

J. R. Eliason

K. L. Kipp

D. E. Olesen

A. E. Reisenauer

D. D. Tillson

R. C. Routson

R. J. Serne

D. R. Friedrichs

S. W. Ahlstrom

R. G. Baca

A. Brandstetter

R. D. Mudd

R. W. Wallace

J. J. Fuquay

A. J. Shuckrow

L. E. Addison

Tech. Information

Tech. Pubs.

8

United Nuclear, Inc.

C. D. Corbit

F. E. Owen 